\title{
Analysis of the Effects of Transcranial Magnetic Stimulation on Functional States and Connectivity of the Human Cerebral Cortex Using Electroencephalography
}

\section{Research Thesis}

Submitted in Partial Fulfillment of the

Requirements for the Degree of Doctor of Philosophy

\author{
Vladimir Litvak
}

Submitted to the Senate of the

Technion - Israel Institute of Technology
AV, 5766 HAIFA AUGUST 2006 
The Research Thesis Was Done Under the Supervision of Prof. Hillel Pratt and Dr. Menashe Zaaroor in the Faculty of Biomedical Engineering

THE GENEROUS FINANCIAL HELP OF THE TECHNION IS GRATEFULLY ACKNOWLEDGED. 


\section{Table of Contents}

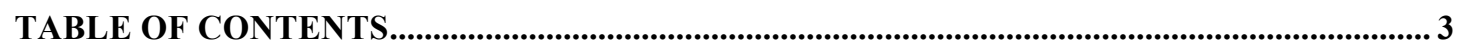

LIST OF FIGURES AND TABLES ........................................................................................... 4

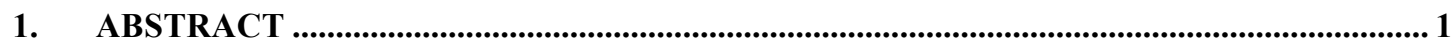

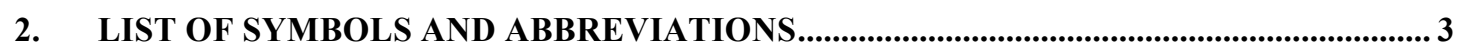

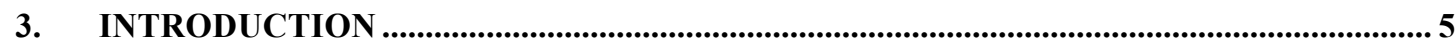

3.1. ELECTRICAL STIMULATION OF THE NERVOUS SYSTEM................................................. 5

3.2. TRANSCRANIAL MAGNETIC STIMULATION (TMS) .......................................................... 9

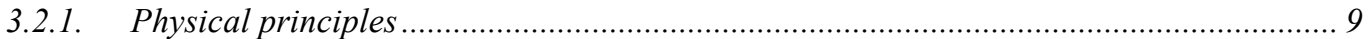

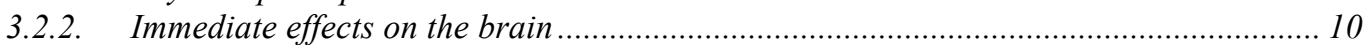

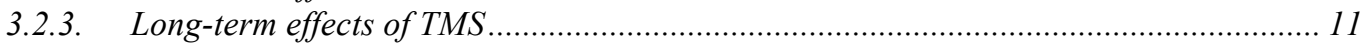

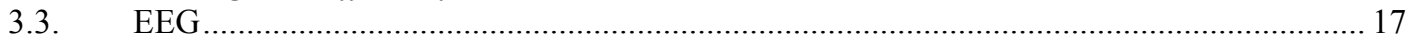

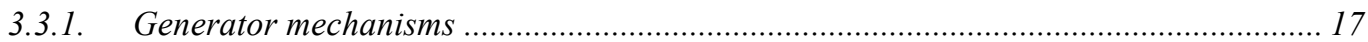

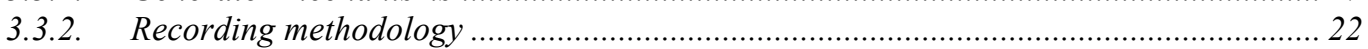

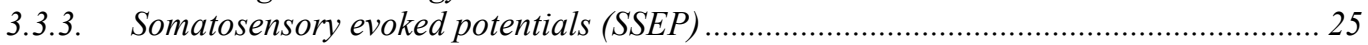

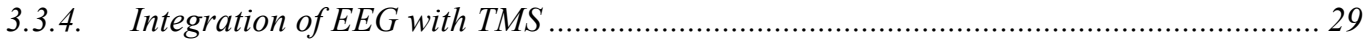

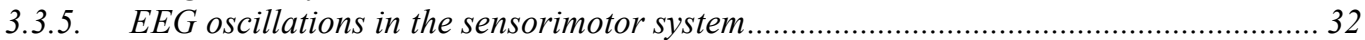

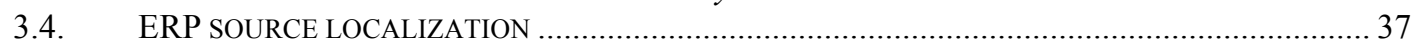

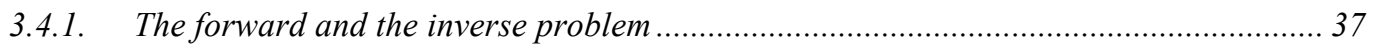

3.4.2. Overview of existing source localization methods .......................................................... 38

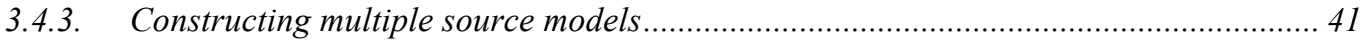

3.4.4. Artifact correction using multiple source models ......................................................... 45

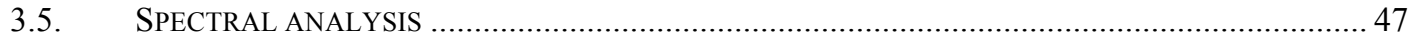

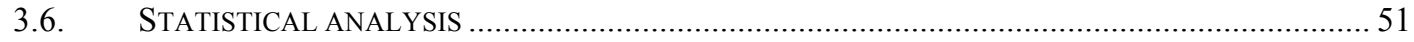

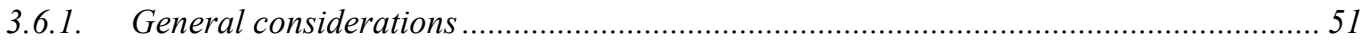

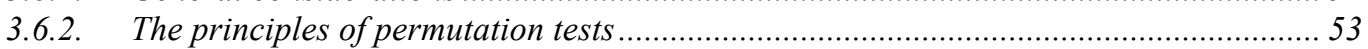

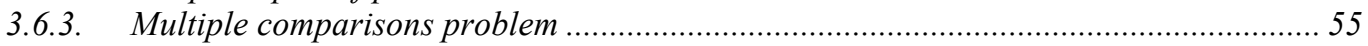

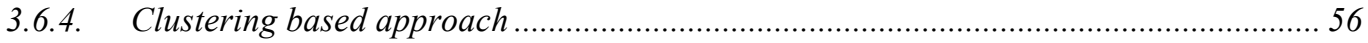

3.6.5. Adapting and extending clustering based tests for specific applications ........................59

4. THE OBJECTIVES OF THE PRESENT RESEARCH...........................................................62

5. DESCRIPTION OF THE STUDIES IN THE PRESENT THESIS ........................................... 64

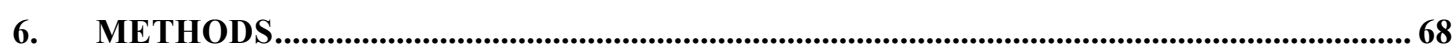

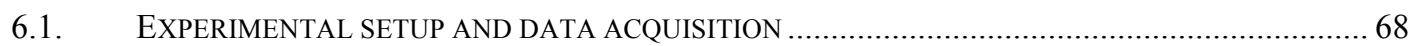

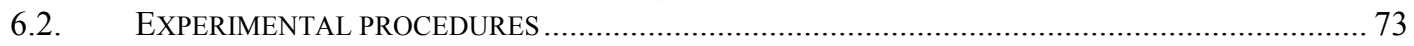

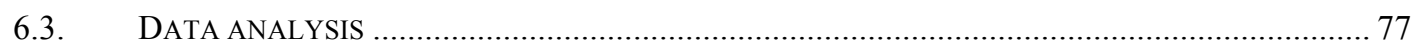

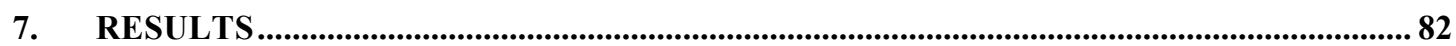

7.1. ARTIFACT CORRECTION AND SOURCE ANALYSIS OF EARLY ELECTROENCEPHALOGRAPHIC RESPONSES EVOKED BY TRANSCRANIAL MAGNETIC STIMULATION OVER PRIMARY MOTOR CORTEX. . 82

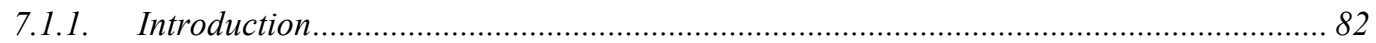

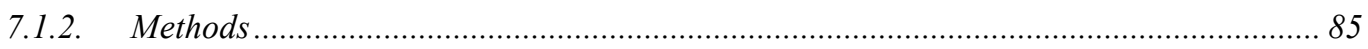

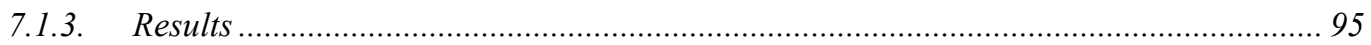

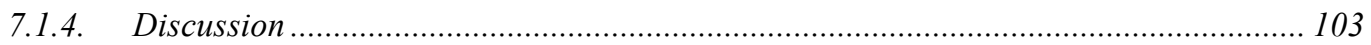

7.2. LTP-LIKE CHANGES INDUCED BY PAIRED ASSOCIATIVE STIMULATION OF THE PRIMARY

SOMATOSENSORY CORTEX IN HUMANS: SOURCE ANALYSIS AND BEHAVIORAL CORRELATES ............ 114

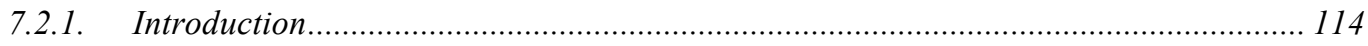

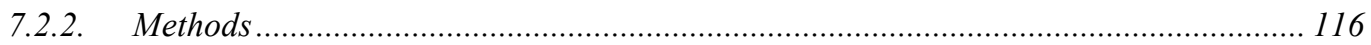

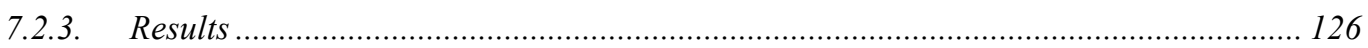

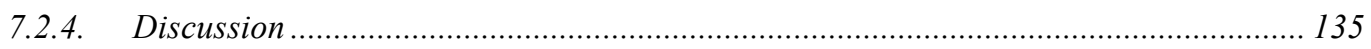

7.3. CHANGES IN AMPLITUDE OF MOTOR EVOKED POTENTIALS ARE CORRELATED WITH THE

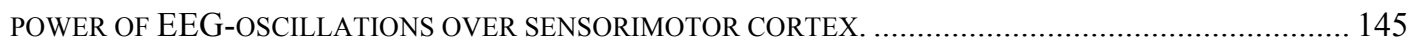

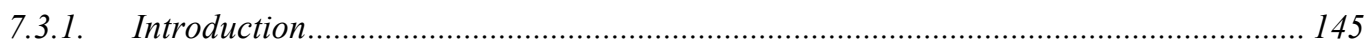

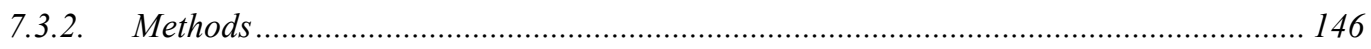

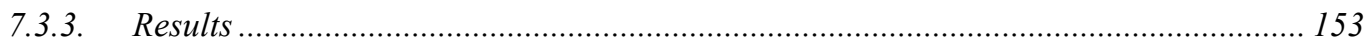

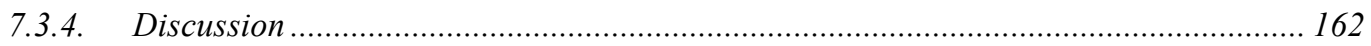

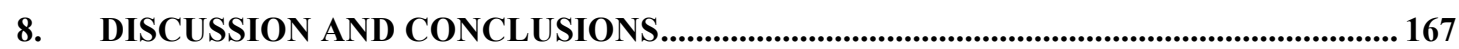

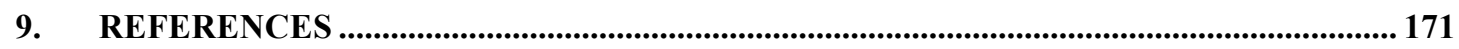




\section{List of Figures and Tables}

FIGURE 1. THE CRITICAL WINDOW FOR SYNAPTIC POTENTIATION AND DEPRESSION.............................. 15

FIGURE 2. EXAMPLES FOR ARTIFACTUAL AND PHYSIOLOGICAL SCALP POTENTIAL DISTRIBUTIONS......... 21

FIGURE 3. MN-SSEP WAVEFORM RECORDED WITH CLINICAL RECORDING SETUP.................................2 28

FIGURE 4. TANGENTIAL+SOMATOSENSORY RADIAL MODEL OF MN-SSEP GENERATION.......................2 29

FIGURE 5. TIME COURSE AND SCALP PATTERN OF ERD/ERS ASSOCIATED WITH HAND MOVEMENT. ....... 35

FIGURE 6. WAVEFORMS AND SPECTRA OF A SERIES OF TAPERS USED FOR MULTITAPER ANALYSIS. ........ 49

FIGURE 7. EXAMPLES OF NEUROIMAGING DATA WHERE HUMAN EYE CAN EASILY DETECT PHYSIOLOGICAL

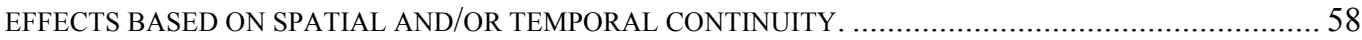

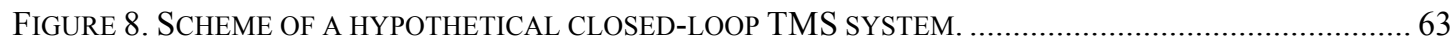

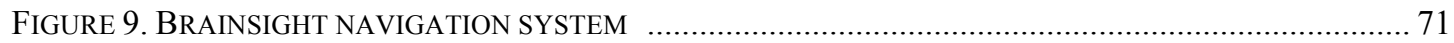

FIGURE 10. EXPERIMENTAL SETUP USED FOR THE PAS STUDY AND MEP VARIABILITY STUDY............. 72

FIGURE 11. GENERAL VIEW OF THE EXPERIMENTAL SETUP DURING AN EXPERIMENT COMBINING MAGNETIC STIMULATION AND EEG RECORDING................................................................... 73

FIGURE 12. DETERMINING THE POSITION OF MOTOR AND SOMATOSENSORY 'HOTSPOT' FOR A PAS EXPERIMENT.

FIGURE 14. ARTIFACT REJECTION INTERFACE OF BESA

FIGURE 15. FLOWCHART FOR ARTIFACT CORRECTION AND ANALYSIS OF TMS-EVOKED ERPS ..............93

FIGURE 16. GRAND AVERAGE OF TMS-EVOKED ERPS BEFORE AND AFTER ARTIFACT CORRECTION.....98

FIGURE 17. PROMINENT SCALP POTENTIAL PATTERNS IN THE ARTIFACT CORRECTED DATA.................. 100

FIGURE 18. THE MULTIPLE SOURCE MODEL FITTED TO THE GRAND AVERAGED DATA AND THE CORRESPONDING SOURCE WAVEFORMS.

FIGURE 19. EEG RESPONSES DEPENDENCE ON STIMULUS INTENSITY OF M1 STIMULATION. ................ 104

FIGURE 20. OVERVIEW SCHEMATIC OF AREAS THAT MAY BE FUNCTIONALLY CONNECTED TO THE PRIMARY MOTOR AREA OF THE CORTEX. .

FIGURE 21 SEMI-SCHEMATIC ILLUSTRATION OF THE TIMING OF VARIOUS PHYSIOLOGICAL EVENTS TRIGGERED BY MEDIAN NERVE STIMULATION AND OF THE DIFFERENT PAS-INTERVENTIONS USED.

FIGURE 22. CHANGES INDUCED BY PAS IN MN-SSEP WAVEFORMS. .

FIGURE 23. PASN20-2.5MS AND PASN20+100MS SIGNIFICANTLY AFFECT MN-SSEP AMPLITUDE BETWEEN 21 AND 31 MS POST-STIMULUS.

FiguRE 24. FITTING OF EQUIVALENT DIPOLES REVEALS A DIFFERENCE BETWEEN PASN20-2.5MS AND PASN20+100MS EFFECTS.

FIGURE 25. ANALYSIS USING BESA MN-SSEP SOURCE MODEL SHOWS THAT PASN20-2.5MS EFFECT CAN BE EXPLAINED BY AN ACTIVITY INCREASE OF THE TANGENTIAL S1 SOURCE.

FIGURE 26. STATISTICAL ANALYSIS OF TWO-POINT DISCRIMINATION DATA ......................................... 136

FIGURE 27. SIGNIFICANT CORRELATION BETWEEN THE CHANGE IN BEHAVIORAL PERFORMANCE AND MODIFICATION OF MN-SSEP AMPLITUDE BY PASN20-2.5MS.

FIGURE 28. MEP AMPLITUDES OBSERVED OVER THE COURSE OF THE EXPERIMENT IN EACH SUBJECT.. 154

FIGURE 29. DIFFERENCES IN PRE-STIMULUS EEG SPECTRA BETWEEN TRIALS WITH HIGH AND LOW MEP AMPLITUDE.

FIGURE 30. THE TIME COURSE OF POWER CHANGES SURROUNDING SELF-INITIATED MOVEMENT OF THE RIGHT THUMB.

FIGURE 31. COMPARISON OF THE EEG RECORDED AROUND THE TIME OF THUMB MOVEMENT TO THE BASELINE PERIOD.

FIGURE 32. THE TIME COURSE OF PRE-TMS POWER DIFFERENCE. 159

FIGURE 33. SCALP CURRENT DENSITY DIFFERENCE PATTERNS FOR THE PRE-TMS DATA AND MOVEMENT DATA.

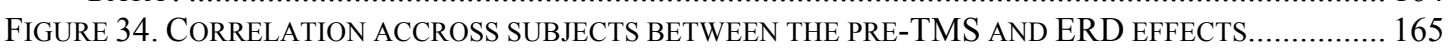

TABLE 1. ELECTROMAGNETIC BRAIN-STIMULATION TECHNIQUES. 


\section{Abstract}

Transcranial magnetic stimulation (TMS) is a safe and noninvasive method for stimulating the human brain based on the principle of electromagnetic induction. When applied to the primary motor cortex TMS evokes electrical responses and contractions in the muscles. It is now used as a diagnostic and therapeutic tool in neurology and psychiatry.

The present research aimed at developing new methods for characterization of the immediate and long-term effects of TMS on the brain and for developing more precise stimulation protocols that would allow purposeful modification of the brain's functional state and connectivity. The ultimate goal of this line of research would be the construction of a closed loop stimulation system that continually modifies the stimulation parameters based on real-time analysis of neural signals. Electroencephalography (EEG) was chosen as the neuroimaging method for the present study due to its high time-resolution and the possibility to combine it with TMS.

In the Results section of the thesis three research projects are presented. The first project was aimed at characterizing responses evoked in the EEG by TMS applied to the left and right primary motor cortex. For this purpose it was necessary to solve the problem of TMS artifacts hampering the analysis of several tens of milliseconds immediately following the stimulus. This was achieved by analyzing data recorded with a specially adapted recording system and subtracting the residual artifact in software. Combining these two approaches enabled almost complete removal of the artifact without distorting physiological brain signals. Based on the artifact-free data, a model of the response that revealed remarkable symmetry between the responses to stimulation at the left and right sides of the brain was suggested.

The second research project examined the long-term effects of TMS. The specific protocol tested - paired associative stimulation (PAS) is an attempt to mimic natural neural learning mechanisms. PAS consists of pairing an electrical stimulus of median nerve at the wrist with magnetic stimulation of the primary somatosensory cortex. It was previously found that a long series of such paired stimuli is capable of modifying the amplitude of a particular peak in the EEG response to median nerve stimulation. 
Our aim was to better understand the mechanism of this modification and test whether it affects the perceptual discrimination ability of the subject. We used a novel nonparametric statistical analysis method and were able to show that PAS affects synapses at superficial layers of cortical area $3 \mathrm{~b}$ responsible for processing of tactile information. We also found significant effects of PAS on behavioral performance and were able to relate them to changes in the EEG response.

The third project addressed the issue of variability of muscular responses to TMS pulses delivered under identical conditions. We showed that this variability is at least partially related to oscillatory activity in the sensorimotor cortical areas. The fact that increase in the power of the oscillations was correlated with a stronger muscular response to TMS challenges the accepted view that oscillations appear during an idling state of the motor system. 


\section{List of Symbols and Abbreviations}

\begin{tabular}{|c|c|}
\hline$\Delta 2 \mathrm{Pt}$ & Change in Two-Point Discrimination Ability \\
\hline ADM & Abductor Digiti Minimi (muscle) \\
\hline AEP & Auditory Evoked Potential \\
\hline AM & Anterior Motor (model source) \\
\hline ANOVA & Analysis of Variance \\
\hline APB & Abductor Policis Brevis (muscle) \\
\hline BA & Brodmann Area \\
\hline BESA & Brain Electrical Source Analysis \\
\hline $\mathrm{Cb}$ & Cerebellum \\
\hline CMA & Cingulate Motor Area \\
\hline $\mathrm{CMC}$ & Cortico-Motor Coherence \\
\hline $\mathrm{CS}$ & Central Sulcus \\
\hline D2 & Index Finger \\
\hline D5 & Little Finger \\
\hline DBS & Deep Brain Stimulation \\
\hline DRG & Dorsal Root Ganglion \\
\hline ECD & Equivalent Current Dipole \\
\hline ECT & Electro-convulsive Therapy \\
\hline EEG & Electroencephalogram/Electroencephalography \\
\hline EMG & Electromyogram/Electromyography \\
\hline ERD & Event Related Desynchronization \\
\hline ERP & Event Related Potential \\
\hline ERS & Event Related Synchronization \\
\hline fMRI & Functional Magnetic Resonance Imaging \\
\hline HFS & High Frequency Stimulation \\
\hline ICA & Independent Component Analysis \\
\hline $\mathrm{L} 2 / 3$ & Cortical Layer $2 / 3$ \\
\hline L4 & Cortical Layer 4 \\
\hline LED & Light Emitting Diode \\
\hline LFP & Local Field Potential \\
\hline LFS & Low Frequency Stimulation \\
\hline LORETA & Low Resolution Electromagnetic Tomography \\
\hline LTD & Long Term Depression \\
\hline LTP & Long Term Potentiation \\
\hline M1 & Primary Motor Cortex \\
\hline $\mathrm{MC}$ & Multiple Comparisons \\
\hline $\mathrm{MCP}$ & Multiple Comparisons Problem \\
\hline MEG & Magnetoencephalography \\
\hline MEP & Motor Evoked Potential \\
\hline MFM & Midline-Frontal-Motor (model source) \\
\hline MLTH & Maximum Likelihood Threshold Hunting \\
\hline $\mathrm{MN}$ & Median Nerve \\
\hline MN-SSEP & Median Nerve Somatosensory Evoked Potential \\
\hline MRI & Magnetic Resonance Imaging \\
\hline PAS & Paired Associative Stimulation \\
\hline PCA & Principal Component Analysis \\
\hline PET & Positron Emission Tomography \\
\hline RMS & Root Mean Square \\
\hline RMT & Resting Motor Threshold \\
\hline
\end{tabular}


rTMS Repetitive Transcranial Magnetic Stimulation

$\mathrm{S} 1$

Primary Somatosensory Cortex

SCD Scalp Current Density

SI

Stimulus Intensity

SMA

Supplementary Motor Area

SNAP

Sensory Nerve Action Potential

SNR

Signal to Noise Ratio

SSEP

Somatosensory Evoked Potential

STDP

STN

Spike Timing Dependent Plasticity

tDCS

Subthalamic Nucleus

TES

Transcranial Direct Current Stimulation

TMS

UN

Transcranial Electrical Stimulation

VEP

Transcranial Magnetic Stimulation

Ulnar Nerve

VPL

Visual Evoked Potential

Ventral Posterolateral Nucleus 


\section{Introduction}

\subsection{Electrical stimulation of the nervous system}

The human brain is one of the most enigmatic systems in the universe. Understanding the brain function and finding ways to treat neurological and psychiatric disorders has interested scientists since ancient times. However, despite the remarkable progress of the 20-th century, neuroscience's most basic questions about the brain remain unanswered. The progress of neuroscience has led to the development of a number of recording and imaging techniques that allow monitoring the brain activity and localizing areas involved in information processing in both healthy and diseased human and animal subjects. Yet, the number of possible ways of medical intervention in neurology and psychiatry is very limited. Neurosurgery can be very effective but for the majority of brain diseases there is no surgical treatment. Pharmacological therapy is widely used as in the other areas of medicine, but the available drugs usually have low specificity and little is known about the biological basis of their action.

Although the exact mechanisms of information processing in the nervous system are not yet completely understood, the fact that this processing is based on electrical activity of excitable cells, the neurons, is well established (Kandel et al., 2000). Therefore, it would be natural to expect that electrical neural stimulation will be widely used in medicine. In fact, until recently the only widely used treatment based on electrical stimulation was the controversial electro-convulsive therapy (ECT) While ECT seems to be effective for treatment of severe depression it is certainly far from being specific, has cognitive side-effects and its mechanism of action is unclear (Challiner and Griffiths, 2000).

In recent years several new stimulation techniques making it possible to affect the central nervous system directly or indirectly have been introduced. The methods presently used in clinical practice or investigated as potential treatments are summarized in Table 1 taken from (George, 2003).

The main limitation of the majority of the methods listed in this table is that their effect cannot be targeted to specific brain areas and controlled in a precise way to attain the treatment goals defined in advance. 
The most effective and specific method used today is the deep brain stimulation (DBS). It is based on implanting stimulating electrodes potentially in any location in the brain. Until now DBS has been effective in treating several severe neurological diseases - epilepsy, Parkinson's disease and dystonia. Research is being carried out in order to extend the applicability of these methods to other neurological and psychiatric disorders. However, the condition of most neurological and psychiatric patients is not severe enough to justify the risk of surgery and the inconveniences associated with carrying implanted devices in their body. In addition, a noninvasive method is necessary to search for potential targets for DBS in humans. Using DBS itself in a trial and error fashion is ethically problematic and inefficient.

An alternative to implanting electrodes into the brain is delivering stimulation noninvasively. Early experiments with electrical stimulation through the skull (Elbert et al., 1981a; Elbert et al., 1981b) were abandoned because of associated pain and later headache. More recently transcranial direct current stimulation (tDCS) was revived (Nitsche and Paulus, 2000) with much lower stimulation intensities. However in its present version the method has only weak modulatory effect on cortical excitability, which limits its range of possible applications.

The pioneering study of Barker et al. (Barker et al., 1985) introduced a novel noninvasive technique of neural stimulation - transcranial magnetic stimulation (TMS). TMS is based on the principle of electromagnetic induction. A brief pulse of electric current is passed through a coil placed next to the subject's head and creates a magnetic field fluctuation which in turn induces electric currents in the brain area below the coil. Since the skull is easily permeated by the magnetic field the intensity of the stimulation can be such that no large currents are induced in the skin of the head and therefore TMS is practically painless. A variety of applications of TMS in both research and medicine have been developed in recent years (Jalinous, 1998; Hallett, 2000; Wassermann and Lisanby, 2001). 
Table 1. Electromagnetic Brain-Stimulation Techniques. From (George, 2003).

\begin{tabular}{|c|c|c|c|c|c|}
\hline Name & $\begin{array}{l}\text { Treatment } \\
\text { Use }\end{array}$ & $\begin{array}{l}\text { Pulse } \\
\text { Delivery }\end{array}$ & $\begin{array}{l}\text { Targeting } \\
\text { Ability }\end{array}$ & Adrantages & Disadvantages \\
\hline $\begin{array}{l}\text { Electro- } \\
\text { convulsive } \\
\text { theraky } \\
(\mathrm{ECT})\end{array}$ & $\begin{array}{l}\text { Qepression, } \\
\text { mania, } \\
\text { catatopia }\end{array}$ & $\begin{array}{l}\text { Skin } \\
\text { electrodes }\end{array}$ & Fair & $\begin{array}{l}\text { Effective for } \\
\text { depression; } \\
\text { side effects } \\
\text { reduced with } \\
\text { newer } \\
\text { systems }\end{array}$ & $\begin{array}{l}\text { Nonfocal; can } \\
\text { lead to memory } \\
\text { side effects; } \\
\text { requires } \\
\text { repeated general } \\
\text { anesthesia }\end{array}$ \\
\hline $\begin{array}{l}\text { Trans- } \\
\text { cttaneous } \\
\text { electrical } \\
\text { nerve } \\
\text { stimulation } \\
\text { (TENS) }\end{array}$ & $\begin{array}{l}\text { Pain, } \\
\text { spasticity }\end{array}$ & $\begin{array}{l}\text { Skin } \\
\text { electrodes } \\
\text { (attached } \\
\text { to } \\
\text { peripheral } \\
\text { nerves) }\end{array}$ & Good & $\begin{array}{l}\text { Poes not } \\
\text { require } \\
\text { surgery }\end{array}$ & $\begin{array}{l}\text { Limited access } \\
\text { to brain }\end{array}$ \\
\hline $\begin{array}{l}\text { Yagus nerve } \\
\text { stimulation } \\
\text { (VNS) }\end{array}$ & $\begin{array}{l}\text { Approved for } \\
\text { epixepsy; } \\
\text { FDA trials } \\
\text { under way for } \\
\text { depression } \\
\text { and anxiety }\end{array}$ & $\begin{array}{l}\text { Electrodes } \\
\text { attached } \\
\text { to vagus } \\
\text { nerve) }\end{array}$ & Fair & $\begin{array}{l}\text { Roes not } \\
\text { invglve brain } \\
\text { surgely }\end{array}$ & $\begin{array}{l}\text { Effects modest } \\
\text { (to date); } \\
\text { uncleanhow to } \\
\text { tune pulses to } \\
\text { alter brain } \\
\text { function }\end{array}$ \\
\hline $\begin{array}{l}\text { Deep brain } \\
\text { stimulation } \\
\text { (DBS) }\end{array}$ & $\begin{array}{l}\text { Approved for } \\
\text { Parkinson's } \\
\text { diseqse; under } \\
\text { investigation } \\
\text { for pain and } \\
\text { obsessive-- } \\
\text { compulsive } \\
\text { disorder }\end{array}$ & $\begin{array}{l}\text { Electrodes } \\
\text { ambed- } \\
\text { ded in } \\
\text { brain } \\
\text { regions) }\end{array}$ & Exce & $\begin{array}{l}\text { Discrete } \\
\text { takgeting; } \\
\text { marked } \\
\text { effects }\end{array}$ & $\begin{array}{l}\text { Potential side } \\
\text { effects if } \\
\text { incokrectly } \\
\text { positioned; } \\
\text { invasive brain } \\
\text { surgery }\end{array}$ \\
\hline $\begin{array}{l}\text { Transcranial } \\
\text { direct } \\
\text { current } \\
\text { stimulation } \\
\text { (tDCS) }\end{array}$ & $\begin{array}{l}\text { Wnder } \\
\text { investigation } \\
\text { for } \\
\text { Parkinson's } \\
\text { disease }\end{array}$ & $\begin{array}{l}\text { Electric } \\
\text { field }\end{array}$ & Unfo & Noninvasive & $\begin{array}{l}\text { Scalp irritation; } \\
\text { nonfocal }\end{array}$ \\
\hline $\begin{array}{l}\text { Transcranial } \\
\text { magnetic } \\
\text { stimuation } \\
\text { (TMS) }\end{array}$ & $\begin{array}{l}\text { Under } \\
\text { investigation } \\
\text { for } \\
\text { depression; } \\
\text { FDA trials } \\
\text { under way }\end{array}$ & $\begin{array}{l}\text { Magnetic } \\
\text { field }\end{array}$ & Exc & $\begin{array}{l}\text { Noninvasive } \\
\text { and safe; } \\
\text { potential for } \\
\text { many } \\
\text { applications }\end{array}$ & $\begin{array}{l}\text { Limited to } \\
\text { surface brain } \\
\text { stimulation; } \\
\text { effect on geural } \\
\text { function stit } \\
\text { unclear }\end{array}$ \\
\hline $\begin{array}{l}\text { Magnetic } \\
\text { seizure } \\
\text { theraky } \\
\text { (MST) }\end{array}$ & $\begin{array}{l}\text { Wnder } \\
\text { investigation } \\
\text { for depression }\end{array}$ & $\begin{array}{l}\text { Magnetic } \\
\text { field }\end{array}$ & Fair & $\begin{array}{l}\text { May offer } \\
\text { better } \\
\text { targeting and } \\
\text { might avgid } \\
\text { side effects of } \\
\text { ECT }\end{array}$ & $\begin{array}{l}\text { No efficacy } \\
\text { datzyet; } \\
\text { requires } \\
\text { repeated } \\
\text { general } \\
\text { anesthesia }\end{array}$ \\
\hline
\end{tabular}


TMS also has a number of drawbacks. The spatial resolution of stimulation is relatively low. The size of the cortical patch in which neurons will fire as a result of a TMS pulse depends on the exact position of the coil relative to the head, coil characteristics, skull anatomy and stimulation intensity (Jalinous, 1998). Typically, currently available coils stimulate an area of cortex a few centimeters in diameter (Thielscher and Kammer, 2002). In addition, it is physically impossible to induce a stronger electric field in the depth of the brain than on its surface with any configuration of coils (Heller and van Hulsteyn, 1992;Ruohonen and Ilmoniemi, 1999). Therefore, TMS is currently limited to superficial cortical regions. However, many functionally important areas such as primary sensory and motor regions, language areas and frontal cortical areas fall into this category.

The research described in the present thesis aims at expanding the applicability of TMS by better understanding its mechanism of action and developing neuroimaging methodology that can potentially be combined with TMS applications in real time yielding more specific and powerful diagnostic and treatment methods for neurological and psychiatric disorders. 


\subsection{Transcranial Magnetic Stimulation (TMS)}

\subsubsection{Physical principles}

Transcranial magnetic stimulation is a method for stimulating the human brain based on the principle of electromagnetic induction. The physical principles behind TMS were first described by Michael Faraday in 1831 (Jalinous, 1998) and later mathematically formulated in Maxwell's equations describing the relation between electricity and magnetism. The basic finding making TMS possible is that fluctuations of electrical current flowing in a conductive medium induce magnetic field proportional to the derivative of the current i.e. to the magnitude of change in the current per time unit. Conversely, fluctuations of magnetic field in a conductive medium induce electrical current in this medium proportional to the time derivative of magnetic field strength. Another critical detail is the fact that magnetic field permeates the human tissues practically without disturbance.

The TMS device based on the principles described above consists of a capacitor charge/discharge system, a thyristor switch capable of switching large currents in a few microseconds and a stimulating coil consisting of one or more tightly wound and well insulated copper coils together with other electronic circuitry such as temperature sensors and safety switches (Jalinous, 1998).Using the charging circuitry the energy storage capacitor is charged to a set level determined by the experimenter. The experimenter then brings the coil to the proximity of the subject's head and triggers the device causing the thyristor switch to conduct the current from the capacitor through the stimulating coil in a very short time of less than a microsecond. This rapid discharge induces a magnetic field around the coil reaching about 2 Tesla at its peak. The buildup and the decay of the magnetic field happening during several hundreds of microseconds are fast enough to induce physiologically effective currents inside the brain. The induced current in the brain flows in the opposite direction to the coil current.

The first TMS coils were circular. In these coils the maximal magnetic field was attained at the perimeter of the circle and therefore the stimulation was ill focused. In order to reduce the stimulated area the double coil (also termed butterfly or figure of eight coil) was introduced. Double coils utilize two windings normally placed side by 
side with maximal field attained at their intersection as a result of superimposing the fields of the two circles.

\subsubsection{Immediate effects on the brain}

The effect of a single TMS pulse is most prominent in the motor system. Stimulation of the primary motor cortex (M1) at a proper location can cause muscle contractions observable both visually and by recording electrical activity of the muscles (electromyogram or EMG) (King and Chiappa, 1989). These muscle responses to magnetic stimulation are called motor evoked potentials (MEP). MEP monitoring is used in neurology as a diagnostic tool for a number of diseases of the central and peripheral nervous system. For instance, it is possible to measure the conductance velocity of the peripheral nerves or assess the extent of damage to the motor cortex inflicted by stroke. TMS can also be used during surgery for monitoring the functional integrity of important neural pathways (Jalinous, 1998). Monitoring changes in MEP parameters (primarily the MEP amplitude) has also been used in research. MEP amplitude is an indicator of the excitability of M1 and in a number of studies TMS was used to monitor changes in excitability over the course of motor tasks (Liepert et al., 1998; Zaaroor et al., 2001; Devanne et al., 2002). In another group of studies TMS was used to detect plastic changes in cortical connectivity (Cohen et al., 1998; Ziemann et al., 2001). Outside the motor system single pulse TMS is much less applicable. It has been used in several studies of the visual system. Single TMS pulses at occipital areas can cause, in some subjects, the perception of light flickers called 'phosphenes' (Kammer, 1999; Kammer et al., 2001). Such pulses can also disrupt visual processing (Kastner et al., 1998; Walsh et al., 1998a; Walsh et al., 1998b; Corthout et al., 2000) creating 'functional lesions' along the pathway. Therefore, TMS can be used to trace visual pathways in the cortex (Cowey and Walsh, 2001).

It is generally accepted that single pulse TMS protocols are very safe and do not cause any plastic changes in the cortex (exceptions to the latter point will be discussed below). Chen et al. (Chen et al., 1997) showed that stimulation at the frequency of 0.1 $\mathrm{Hz}$ does not change cortical excitability. 


\subsubsection{Long-term effects of TMS}

\subsubsection{Repetitive TMS (rTMS)}

Single-pulse TMS is a useful tool for probing the cortical excitability and precise disruption of cortical processing. However, for both research and therapy it was necessary to also develop stimulation methods capable of modifying the neural excitability and connectivity for a period outlasting the stimulation itself.

The ideas for developing such methods originated from studies at the level of single cells, tissue cultures and brain slices originating in the early 1970's. The basic theory behind these studies is even older and is rooted in the work of the Russian physiologist Ivan Pavlov at the beginning of the $20^{\text {th }}$ century and of the Canadian psychologist Donald Hebb in the 1940s. Pavlov and Hebb suggested that repeated simultaneous activation of two neural populations can lead to strengthening of the connection between them. The most famous formulation of this principle is know as the Hebb's law and appeared in Hebbs's book "The organization of behavior" in 1946.

"When an axon of cell A is near enough to excite cell B and repeatedly or persistently takes part in firing it, some growth process or metabolic change takes place in one or both cells such that A's efficiency, as one of the cells firing B, is increased."

This is more often paraphrased as "Neurons that fire together wire together". The first physiological manifestation of the Hebb's law was found in experiments on hippocampal slices. In these experiments response to stimulation of fibers innervating the hippocampus was increased following a train of high-frequency stimuli $(10 \mathrm{~Hz}$ and above). The increase lasted for $30 \mathrm{~min}$ to 10 hours. This phenomenon was name long-term potentiation of synaptic efficacy (LTP). LTP can be induced not only by high-frequency stimulation but also by pairing of stimuli delivered at pre-synaptic and post-synaptic sites. The opposite phenomenon of long-term depression (LTD) can also be induced by applying stimulation at low frequencies of about $1 \mathrm{~Hz}$. LTP and LTD have been extensively studied on levels ranging from molecular to behavioral and are widely believed to be the neural substrate of learning and memory.

There is a variety of protocols for inducing LTP/LTD in different neural systems. The most straightforward of them that can be applied to TMS is the delivery of stimulation 
trains at high frequency for induction of LTP and at low frequency for induction of LTD. It was indeed found that in the motor cortex higher rates (above $10 \mathrm{~Hz}$ ) produce an increase in MEP amplitude while lower rates $(1 \mathrm{~Hz})$ have the opposite effect (Pascual-Leone et al., 1994; Wassermann et al., 1996; Chen et al., 1997; Muellbacher et al., 2000). Thus, since the mid 90's a distinction emerged in the literature between single pulse TMS and repetitive TMS (rTMS). The difference between the two techniques is primarily in the frequency of magnetic pulse delivery. rTMS is usually defined as stimulation at a rate of $1 \mathrm{~Hz}$ and above. Unlike single pulse TMS, rTMS can produce observable effects at various cortical sites. These effects usually last for at least a few minutes and sometimes can last for up to an hour. Depending on stimulation parameters rTMS can either excite or inhibit the stimulated area. The long-term effects of rTMS were used to induce 'virtual lesions' in various cortical areas. rTMS is the only tool that allows disruption of activity of a specific cortical area in a temporary and reversible way in a fully awake healthy human subject. This turns rTMS into a valuable tool in both clinical neuroscience and cognitive psychology (Walsh and Rushworth, 1999; Hallett, 2000; Pascual Leone et al., 2000). For instance, it is possible to produce reversible speech disruption similar to classical aphasias by stimulating cortical language centers (Pascual-Leone et al., 1991). This is used both for the study of language and for determining the dominant hemisphere before neurosurgery (Epstein, 1999). rTMS has also been used in psychiatry as an alternative to ECT for treatment of depression and bipolar disorder (Wassermann and Lisanby, 2001). Full survey of possible applications of rTMS is beyond the scope of the current discussion. It would suffice to say that rTMS can produce relatively longlasting effects. The behavioral expression of these effects depends on the area being stimulated and on the stimulation parameters.

\subsubsection{Paired associative stimulation (PAS)}

The main disadvantage of traditional rTMS techniques is their low specificity. rTMS modifies the excitability of cortical tissue and the experimenter can control the location where rTMS is applied and to some extent the direction of the change by choosing the stimulation protocol. But by just delivering stimulation trains it is not possible to specifically modify the connectivity between pre-defined populations of neurons. The possible solution was again indicated by basic LTP/LTD research. Starting in the mid 1990s several studies have shown that a synaptic connection between two single neurons can be modified in more specific way by controlling the 
timing rather than frequency of their action. LTP is induced when the postsynaptic neuron is repeatedly activated within a short time window of several tens of milliseconds after the activation of the presynaptic neuron. When the order of activation is reversed, this results in induction of LTD. This variant of Hebb's law is known in the literature as 'Temporally Assymetric Hebbian Learning Rule" or "Spike Timing Dependent Plasticity" (STDP) (Markram et al., 1997; Zhang et al., 1998).

Several difficulties arise when trying to implement STDP in human subjects with TMS. While STDP was most clearly demonstrated by recoding and stimulating single neurons, TMS has much lower spatial resolution and can only stimulate large neural populations. In addition, the two stimuli must be delivered at close temporal proximity. This short time interval does not allow moving the coil to stimulate preand postsynaptic populations located at distinct cortical sites. Possible solutions are using two coils or pairing TMS with a different kind of stimulation. The latter idea was exploited in one of the studies presented in the present thesis and will therefore be discussed more extensively here.

In the study of Stefan et al. (Stefan et al., 2000) magnetic stimuli to the hand motor area were paired with electrical stimulation of the median nerve at the wrist and the effect of a series of such paired stimuli on the amplitude of MEP was examined. This particular protocol is a natural choice based on considerations described above. If TMS has to be paired with another kind of stimulation that activates cortical neurons, sensory stimulation would be the obvious candidate. In order to assess the effect of pairing, the change in activation of the same neural population as the one affected by this protocol must be measured. MEPs are a convenient way to do just that because they are easy to measure and by maintaining constant coil position during MEP measurement and pairing intervention one can make sure that the same restricted cortical area is probed by measuring MEPs and modified by pairing. These considerations restrict us to working with the primary motor cortex where MEPs can be elicited. Therefore, the sensory modality chosen for pairing must be the one that affects the motor cortex as directly as possible. For this reason the somatosensory modality was chosen. Although the present consensus among anatomists is that M1 does not receive input from the discriminative somatosensory pathway directly (Jones, 1986; Darian-Smith et al., 1993), there is massive connectivity between the primary somatosensory cortex (S1) and M1 and there is experimental evidence that 
M1 is activated by somatosensory stimulation at very short latencies (Huang et al., 2000; Balzamo et al., 2004). A simple way to apply well defined somatosensory stimulation is to electrically stimulate one of the two nerves innervating the palm of the hand. The median nerve provides sensory innervation for the palmar surface of the thumb, $2^{\text {nd }}, 3^{\text {rd }}$ and medial half of $4^{\text {th }}$ finger. The ulnar nerve innervates the lateral half of the $4^{\text {th }}$ finger and the $5^{\text {th }}$ finger and the body of the hand below these fingers, on both the palmar and dorsal surfaces of the hand. Like other peripheral nerves the median and ulnar nerves contain sensory fibers of three types: (1) proprioceptive receptors from skeletal muscles, tendons and joint capsules, (2) exteroceptive receptors for touch and pressure and (3) receptors for heat, cold and pain. Electrical stimulation of these nerves, especially the median nerve, is used routinely in clinical practice for recording somatosensory evoked potentials (SSEP) (Nuwer et al., 1994b).

One of the central challenges in designing an intervention protocol involving pairing between electrical stimulation of peripheral nerve and magnetic stimulation of the cortex is finding the optimal time interval between the two stimuli for inducing the maximal effect. After extensive testing of many intervals Stefan et al. (Stefan et al., 2000) found that when the magnetic stimulus was delivered $25 \mathrm{~ms}$ after the peripheral electric stimulus, a long lasting increase in MEP amplitude was induced after repeating the pairings every $20 \mathrm{sec}$ for half an hour. This pairing protocol was named by the authors "paired associative stimulation" or PAS. In an additional study Stefan et al. performed a number of tests aimed at further clarification of the mechanisms of PAS at the cellular level (Stefan et al., 2002). Their conclusion was that the properties of PAS are consistent with the hypothesis that PAS effect on MEP amplitude is a systemic manifestation of spike timing dependent plasticity at the cellular level. This hypothesis predicts that there should also be an interval range where MEP amplitude will be decreased by the pairing. This prediction was indeed confirmed by Wolters et al. (Wolters et al., 2003). They found that when TMS is delivered $10 \mathrm{~ms}$ before the median nerve stimulation, a decrease in MEP amplitude is induced. Based on testing intermediate intervals as well, a curve of the dependence of the effect on the PAS interval was presented. This curve looks very similar to STDP curves derived from other neural systems (Figure 1). As could be expected, in behaving humans the phenomenon is more complex than in brain slices. For instance Stefan et al. (Stefan et al., 2004) found that it can be suppressed when the subject's attention is directed away from the stimulated hand. Since its development, the PAS protocol at M1 has 
been used in several studies in order to assess cortical plasticity under different conditions. Following motor learning the ability to induce an increase in MEP by PAS has benn shown to be reduced whereas the ability to induce a decrease is enhanced (Ziemann et al., 2004; Stefan et al., 2005). This phenomenon is also observed with LTP/LTD in animals. Additional findings were that the PAS effect is stronger in dystonic patients (Quartarone et al., 2003), but is blocked in Parkinson's disease patients (Morgante et al., 2006; Ueki et al., 2006). Since the PAS protocol was introduced relatively recently, additional studies are expected to expand its range of application but already now it is clear that PAS can be useful for research, clinical diagnostics and possibly treatment.
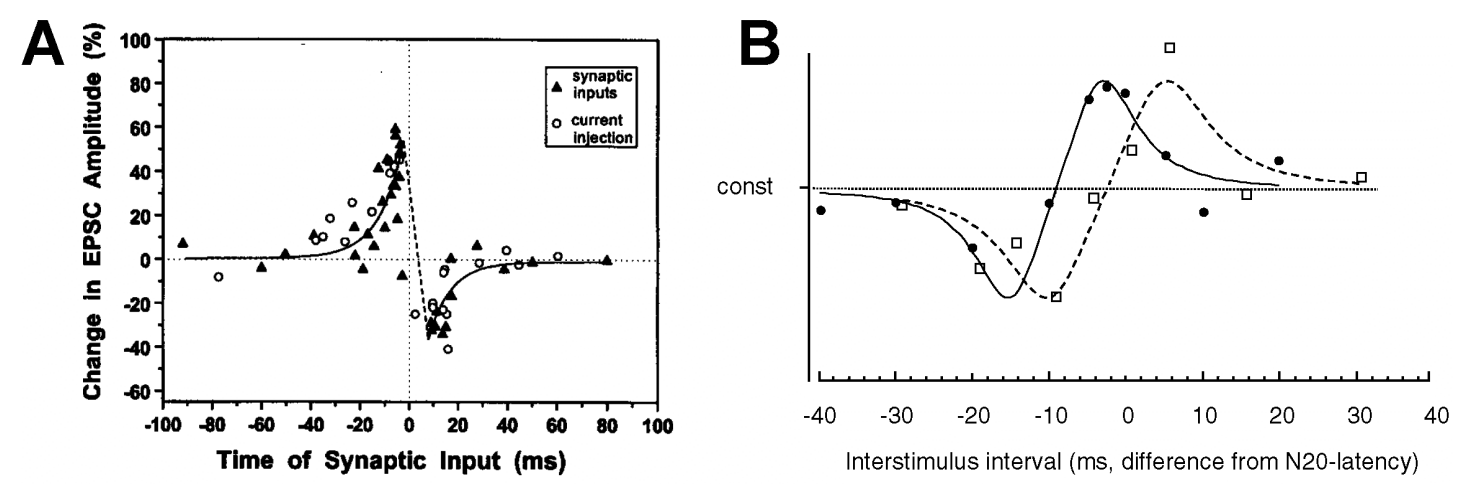

Figure 1. The critical window for synaptic potentiation and depression. A. In the frog tectum (adapted from (Zhang et al., 1998)) B. In the human somatosensory (full circles) and motor (empty squares) system (adapted from (Wolters et al., 2005)). In all cases when the synaptic input precedes the postsynaptic activation the subsequent effect of this input on the postsynaptic cell is increased. Conversely, when the synaptic input follows the presynaptic activation, its subsequent effect is decreased. The directions of the graphs in $A$ and $B$ are reversed because the $X$-axis in $A$ shows the timing of the synaptic input relatively to the postsynaptic spike whereas the $\mathrm{X}$-axis in $\mathrm{B}$ shows the time of the postsynaptic activation (TMS) relatively to synaptic input (N20).

An important direction for further research is expanding the applicability of PAS and similar protocols to areas of the cortex other than M1. A step in this direction was recently taken by Wolters et al. (Wolters et al., 2005). They introduced a version of PAS where instead of M1 the magnetic stimulation is delivered at S1 (defined just as the area $2 \mathrm{~cm}$ posterior to the M1 spot where MEPs are elicited). In a sense this protocol is more straightforward than the original M1 PAS, since TMS is delivered to precisely the area receiving direct median nerve input. Part of the present thesis is a study aimed at characterizing the underlying mechanisms of the S1 PAS with multichannel EEG. Since some background about EEG and somatosensory evoked potentials is required to discuss the S1 version of PAS it will be discussed in more details after these topics are introduced. 
In conclusion, TMS can serve two different purposes. It can cause changes in cortical connectivity and it can also be used to reveal such changes. In fact, these two functions are conceptually unrelated. Thus, connectivity modulation by TMS can be studied using means other than TMS itself (such as EEG), and TMS can be used to study connectivity changes induced by other causes. It is also possible to use TMS for evaluating TMS-induced plasticity but it is solely a matter of convenience. A similar situation exists, for instance, in intracellular recordings where it is convenient to inject current and measure the cell's response with the same electrode but these two functions can also be separated if necessary. 


\subsection{EEG}

Electroencephalography (EEG) is the main neuroimaging method used for the studies in the present thesis. In this chapter this technique will be discussed in details starting with the underlying physiological mechanisms and the recording methodology. At a later stage more specific issues related to the present thesis, somatosensory evoked potentials and integration of EEG and TMS, will be discussed.

\subsubsection{Generator mechanisms}

Large parts of the human brain consist of densely packed excitable cells processing information by conducting currents through their membranes. Therefore, electrical activity is an abundant phenomenon in the living brain tissue. However, most of this activity cannot be measured non-invasively. The brain is surrounded by several layers of protective tissue, most notably by the thick skull which is a poor conductor of electricity. Only when weak electric fields generated simultaneously by large number of cells undergo spatial summation, the total field is strong enough to be detectable by sensors located outside the skull. Luckily for the researchers, the cytoarchitecture of the cerebral cortex makes such summation possible.

When observing a properly stained piece of cortical tissue under the microscope one notices immediately the array of identically oriented cells with triangularly shaped soma, a single apical dendrite extending towards the cortical surface, multiple basal dendrites, and a single axon. These are pyramidal cells, the excitatory projection neurons of the cerebral cortex (Kandel et al., 2000). Pyramidal cells transmit information to neighboring and distal cortical areas and to subcortical structures via action potentials. Action potentials are robust and generate relatively strong currents but their properties prevent their summation in a manner that will create electrical field visible from outside the skull. Although action potentials propagate to great distances the currents they generate are local. In the case of myelinated axon they flow mainly between the neighboring nodes of Ranvier. Since the pyramidal cells do not fire in complete synchrony, even when there is a bundle of axons sharing common origin the process associated with propagation of an action potential will occur at different locations in different fibers, thus preventing summation. Conversely, 
synaptic currents generated by densely packed cell bodies and dendrites all occur at the same place can therefore undergo spatial summation much more effectively.

A pyramidal cell can receive inputs via synapses located either at the cell body and the basal dendrites or at the apical dendrite. Let us consider the particular case of excitatory input close to the soma. Release of neurotransmitter from excitatory synapses leads to opening of post-synaptic channels and positive ionic currents enters the cell. As a result a voltage gradient is formed where the cell body is more positive than the apical dendrite and current flows along this gradient to the apical arbor and returns via extra-cellular medium. This process results in formation of positively charged source at the apical region and negatively charged sink at the basal region of the cell.

When a pyramidal cell receives excitatory input at its apical arbor a similar sequence of events occurs leading to the opposite result. Since the apical arbor becomes depolarized relative to the cell body, the current flows in the opposite direction and the positive source and negative sink are formed at the basal and apical regions respectively.

When considering inhibitory inputs the signs are reversed once again so that the consequences of inhibitory input at the cell body are similar to the consequences of excitatory input at the apical tree and vice versa.

In practice, each pyramidal cell is bombarded with multitude of synaptic inputs both excitatory and inhibitory at all its parts at each moment (Shadlen and Newsome, 1998). The overall pattern that will be seen is the results of summation of all the influences described above. When an array of pyramidal cells at a particular patch of cortex is activated synchronously by inputs exerting similar influence at all the cells, the electrical fields and associated currents generated by them sum up because all the apical trees are aligned in the same direction. The total current generated by the patch is considerable and can be easily measured by intracortical electrode as local field potential (LFP). Currents flowing between cortical layers can be studies with the method of current source-density analysis. Mitzdorf (Mitzdorf, 1985) reviewed numerous studies performed in different animal models and different cortical regions and described the basic current pattern characteristic of cortical activation by sensory 
input and seen in all the sensory areas examined. At the first stage excitatory thalamic input arrives at cell bodies and basal dendrites of pyramidal neurons at cortical layer 4 (L 4). Electrode located above the cortical surface records positive voltage deflection at this stage for reasons discussed above. This response stage is called 'primary positivity' (Allison et al., 1991). At the second response stage the excitation propagates via cortical interneurons to the upper layers of the cortex - layer $2 / 3$ (L $2 / 3$ ). This leads eventually do activation of apical dendrites of L 4 pyramidal cells reaching into $\mathrm{L} 2 / 3$. At this time negative voltage deflection is recorded at the surface named 'primary negativity'. Further cortical processing results in a series of additional voltage deflection whose timecourse is specific to the species and cortical area examined but the initial positivity-negativity sequence seems to be universal.

The currents associated with cortical processing flow primarily inside the brain. Only fractions of them exit the skull and flow on the scalp due to high resistance of this pathway. But these small currents are sufficient to generate scalp voltage gradients that can be recorded by an array of sensors attached to the scalp as will be discussed in details below. The changes of these voltage gradients over time constitute the electroencephalographic signal. For the reasons described above the pyramidal cells are the major contributor to this signal although other types of cells might be equally important for information processing in the cortex. But of all cortical cell types pyramidal cells are not a bad choice for monitoring since the information they process is what eventually is transmitted to other areas. Thus there is a reason to assume that all important processing events will be reflected in their activity.

Although pyramidal cells are responsible for most of EEG activity, there are also neural events of a different origin observable with EEG. In particular, during conduction of action potential in sensory nerves within the head, voltage gradients are sometimes generated on the scalp when the activation volley passes through junctions of regions with different conductive properties. For instance, Martin et al. have demonstrated that during early auditory processing specific scalp activity is generated when action potentials pass through the boundary between the region where auditory nerve is submerged in cerebrospinal fluid and the region where it passes through the temporal bone (Martin et al., 1995). 
A point that is of great importance for many of the discussions that will follow is that because of the specific generation mechanisms described above the voltage patterns generated on the scalp by physiological EEG sources are always dipolar. They consist of the source - scalp location where the positive current exits the skull and the sink scalp location where it enters back. The source and the sink are located on the opposite sides of the cortical generator. Depending on the location of the generator in the brain and the sensor array used for EEG recordings, there are cases where both the source and the sink are visible to the sensors and other cases where only one of them is visible. However, observing a dipolar pattern either by measuring voltage directly or after some more complicated signal processing is a strong indication for existence of underlying physiological source. Monopolar patterns affecting several sensors are also likely to have physiological origin, but effects seen only at a single sensor or patchy effects seen at non-adjacent sensors are likely to be non-physiological artifacts. Some examples for physiological and artifactual patterns are shown at Figure 2. 

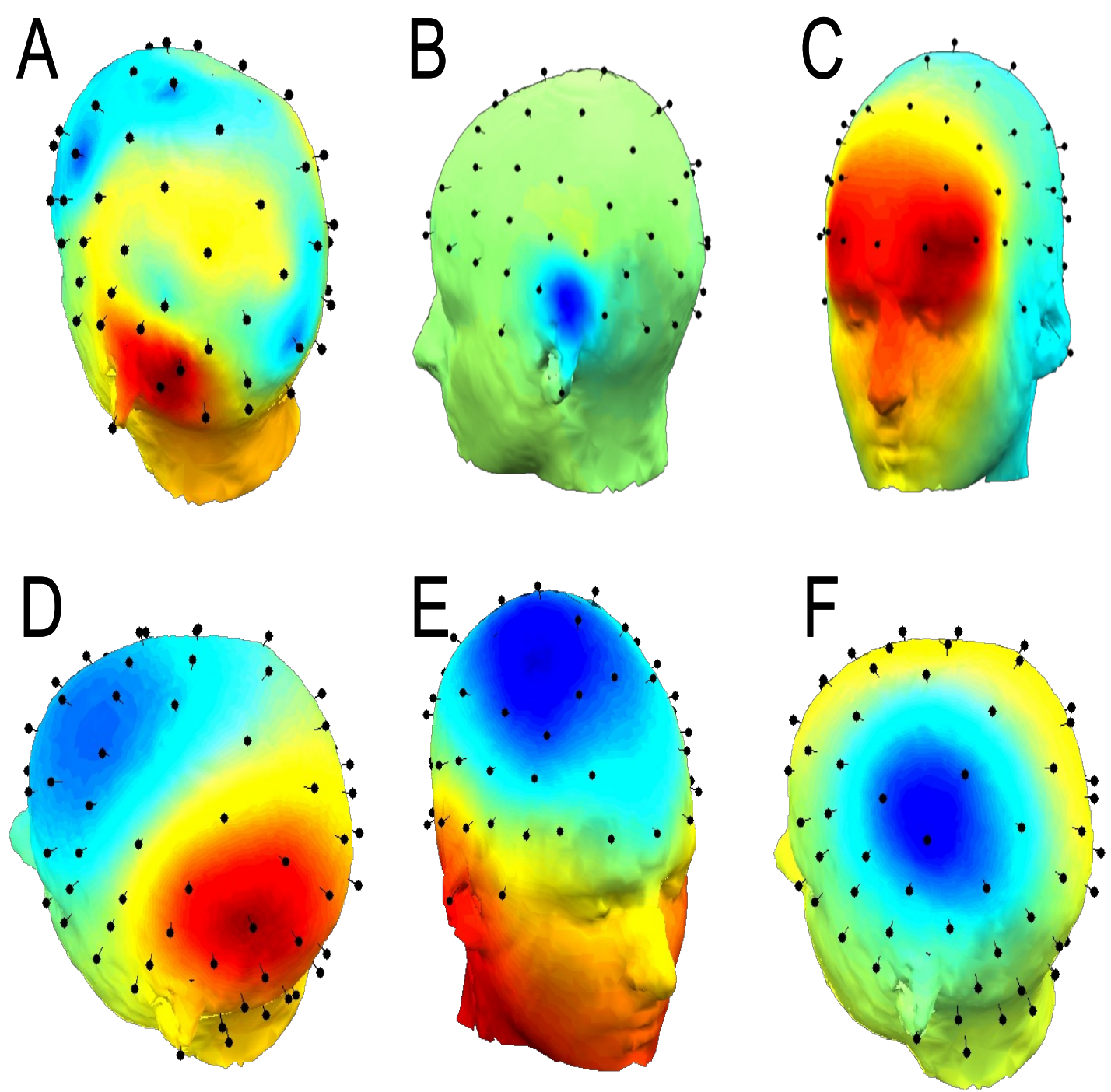

Figure 2. Examples for artifactual (top row) and physiological (bottom row) scalp potential distributions. The color scale is arbitrary. A. Measurement noise $\mathbf{B}$. Temporal muscle noise $\mathbf{C}$. Eye blink D. Tangential source around the central sulcus E. Deep source oriented toward the vertex F. Radial postcentral source 


\subsubsection{Recording methodology}

EEG recording in general and study of event related potentials (ERP) in particular, are well established research methods in neuroscience, medicine and psychology (Gevins and Remond, 1987). The basic EEG technique involves attaching an array of electrodes to the subject's scalp and amplifying the potential difference between each of these electrodes and a reference electrode by a factor of approximately 200,000 . In order to increase the signal to noise ratio it is usually necessary to reduce the impedance between the electrodes and the skin. To achieve this, the skin is prepared by abrasive paste and conductive gel is injected in the cleft between the skin and the electrode.

The electrodes can be attached to the skin either one by one using skin-friendly glue that is dissolved at the end of the experiment or as an array fixed to elastic cap. In order to enable comparison between different studies standard systems for electrode placement have been suggested (Jasper, 1958; Chatrian et al., 1988; Oostenveld and Praamstra, 2001). The most popular of these is the extended 10-20 system (Chatrian et al., 1988) that is presently accepted as standard (Nuwer et al., 1994a). The elastic caps have the advantage of placing the electrodes at approximately correct positions without any special measurements needed. Their drawback is that sometimes skin preparation requires more effort but the EEG systems and caps developed in the recent years make the process much faster. In all studies presented below caps were used for electrode placement. Although caps provide approximately correct electrode placement, it is advisable to measure electrode positions for each experiment in order to account for possible cap shifts or differences due to subject head size variability.

Since EEG signal reflects differences in voltage, EEG cannot be recorded with just one electrode. Even to create a single recording channel, two electrodes are necessary one of which is reference. But where should these electrodes be located? We are definitely interested at looking at the electrode overlying the brain region of interest, but what should its voltage be compared to? In physics potential is defined relative to infinity and for practical purpose a point in the lab distant from the subject can be taken as a reference. But this turned out to be a bad solution, since the voltage on the scalp is influenced by sources other than the brain activity such as electrical activity of the heart and electromagnetic emissions of devices in the lab. The further the 
reference electrode is from the recording site the more likely are the artifact sources of no interest to influence the potential difference between the reference and the recording electrode. Therefore, to reduce artifact contamination the reference should be close to the recording channels. On the other hand, in the extreme case when the reference and the recording electrode coincide, their voltage difference will always be zero and they will record nothing. The solution used in practice is in the majority of cases a cephalic reference, i.e. reference electrode fixed somewhere on the head. The exact location should be such that the reference will not pick noise and thus contaminate the recording. So locations without muscles such as the nose or the chin are good possibilities. Some researchers use linked earlobes as a reference but this practice is unsuitable for many purposes, particularly source localization, because it distorts the distribution of potentials on the scalp by electrically shortening two remote points. Another possibility is using the average of all the channels as a reference which has the disadvantage of producing a signal where every channel is influenced to some albeit minor extent by all others. In case of multichannel recordings where all the channels were recorded with the same reference, a reference can be changed offline by recomputing. For instance by subtracting the signal of one channel from all others, this channel is turned into reference. As mentioned above, physiological events induce dipolar voltage patterns on the scalp. When the two electrodes happen to overlie the opposite poles of such a pattern, the voltage difference between them will be large and they will record the activity of the brain source creating the pattern very clearly. In ERP a clear peak will usually be seen. On the other hand when the two electrodes overlie the same pole of the dipole they will record the signal of the underlying source only weakly or not at all. Therefore, when only a few recording channels are used, the placement of the recording electrodes and the reference is crucial for determining their selectivity for detecting particular brain sources and their ability to differentiate between sources inducing similar scalp voltage patterns. Conversely, in multichannel recording, when electrodes cover the whole scalp, sources not resolved by a particular electrode may be resolved by others and this fact diminishes the importance of reference location. Further discussion of this issue appears in (Nunez, 1981) and (Michel et al., 2004). The bottom line is that for localizing the sources of EEG signals in the brain the position of reference does not matter as long as all the channels are referenced to the same single location (or the average). The reference can matter for other kinds of analysis such as phase analysis but these analyses were not used in the present thesis. An additional useful derivation 
that is not suitable for source localization but may substitute it altogether in some sense is the Laplacian derivation (Hjorth, 1991). In this method the signal assigned to each channel is a difference between the originally recorded signal and weighted average of the neighboring channels (were the weighting is function of their distance from the electrode of interest). This derivation sharpens the potential map by stressing sharp changes and attenuating patterns weakly affecting many electrodes. Thus, the contribution of each brain area will only be seen at electrodes located above it on the scalp. The disadvantage of this method is that it may completely miss sources located in walls of cortical sulci and projecting tangentially to the scalp surface. Such sources affect electrodes distant from their actual location in a diffuse way and the patterns they create are attenuated by the Laplacian derivation. In addition, sharpening of scalp maps increases inter-subject variability and may hamper statistical analysis of scalp maps because physiologically analogous sources in different subjects will be more likely to have non-overlapping scalp signatures.

For many years the main method of EEG analysis has been inspection by eye performed by a trained physician. Such an inspection allows detecting signs of epileptic seizures and brain tumors and differentiating between different brain states (such as sleep stages). A more sophisticated analysis technique is averaging of EEG signals time locked to some event (such as sensory stimulus) over multiple trials. Such averaging helps to reveal components in the EEG signals that are time-locked to the event (e.g., activity evoked by the event in question). These components are event related potentials (ERP). Analysis of ERP's has typically focused on amplitudes and latencies of peaks distinguishable in an ERP waveform. In recent years the EEG field attracted some renewed interest due to introduction of novel signal analysis techniques such as independent component analysis (ICA) (Makeig et al., 1997; Kobayashi et al., 1999; Vigario et al., 2000), wavelet analysis (Lachaux et al., 2002) and spectral analysis (Rappelsberger, 1998). Also more advanced techniques have been developed that solve the problem of source localization enabling to pinpoint the location of areas involved in generation of different ERP components (Mosher et al., 1992; Miltner et al., 1994; Pascual Marqui et al., 1994; Scherg and Berg, 1996). An idea being intensively developed right now is combining the high spatial resolution of fMRI with the superior temporal resolution of EEG to learn both the location of areas activated and the exact sequence of their activation (Mulert et al., 2002; Vitacco et al., 2002). 


\subsubsection{Somatosensory evoked potentials (SSEP)}

Recording of somatosensory evoked potentials are a representative example of EEG/ERP methodology described above. SSEPs are also the central subject of one of the studies presented below. They will therefore be discussed here in more detail.

As mentioned above, SSEPs can be elicited by stimulating the arm either above the median nerve (MN-SSEPs) or the ulnar nerve (UN-SSEPs). These nerves contain both fibers transmitting sensory information and nociceptive fibers transmitting information about painful stimuli. These two categories of fibers transmit information to the brain via two different sensory pathways: discriminative ascending path and anterolateral spinothalamic tract (Kandel et al., 2000). Conveniently for the researchers, the sensory threshold for activating the nociceptive pathway is higher than the threshold for activating the sensory pathway. This makes it possible to stimulate the sensory pathway without causing substantial discomfort to the subject. Therefore, we will focus on the discriminative path since this is the path relevant for the study presented below.

Discriminative ascending path (or the lemniscal pathway) conveys conscious sensory information about touch, heat and cold and also proprioceptive information from muscles and joints. The sensory neurons located in dorsal root ganglia (DRG) send their fibers into the spinal cord where they ascend to medulla and make the first synapse there in nucleus gracilis/cuneatus. The axons of medullar interneurons cross to the contralateral side in medial lemniscus and make the second synapse of the pathway in the ventral posterolateral nucleus (VPL) of the thalamus. This nucleus in turn sends its output to S1. There seems to exist separation between different somatosensory modalities all along the somatosensory pathway resulting in separate cortical areas being innervated by different kinds of input (Jones, 1986). Area 3b which is located at the posterior wall of the central sulcus (CS) and area 1 which is located immediately posterior to $3 \mathrm{~b}$ at the anterior edge of the postcentral gyrus are the primary recipients of the cutaneous input - the sense of touch. Area $3 \mathrm{a}$ which is located deep at the bottom of the central sulcus and area 2 at the posterior edge of the postcentral gyrus are the primary recipients of ‘deep' input - from muscles and joints. 
An interesting anatomical point important for the following discussion is the existence of anatomical marker for the representation of the hand in S1 and M1 fed by the median nerve (Yousry et al., 1997). This is a knob-like structure that is shaped like an omega or epsilon in the axial plane and like a hook in the sagittal plane of the MRI. On the cortical surface of cadaver specimens this precentral knob corresponded precisely to the characteristic 'middle knee' of the central sulcus that has been described by various anatomists in the last century. It was confirmed both with stereotactically guided transcranial magnetic stimulation (TMS) and functional MRI that this "hand knob" contains the motor neurons involved in the control of hand and finger movements. The sensory representation of the hand and fingers is assumed to be located at the areas of the postcentral gyrus immediately posterior to this knob.

In order to elicit SSEPs MN or UN can be stimulated by placing two electrodes at the wrist (for $\mathrm{MN}$ ) or at the lateral surface of the hand (UN) and passing short current pulses between them. These pulses evoke action potentials in multiple axons simultaneously and the resulting volleys of activation propagate both in the direction of the spinal cord and in the direction of nerve endings in the skin. The latter phenomenon can be used to monitor the efficiency of the simulation. This is done in two ways. First, the stimulation activates both sensory and motor axons, though the sensory threshold is lower. Activation of the motor fibers leads to muscle twitches that that can be observed by the experimenter. Since the motor threshold is higher than the sensory one, the muscle twitches indicate that the sensory component of the nerve is also stimulated. However, this rule of thumb is sometimes insufficient to verify that the stimulation intensity stays constant throughout an experiment. For this purpose sensory nerve action potentials (SNAPs) elicited antidromically in sensory nerve endings can be monitored with ring electrodes fixed on the fingers.

While antidromic activation of the sensory fibres can be used for monitoring stimulation intensity, the actual SSEPs are elicited by the orthodromic volley. This volley propagates via the sensory neurons in the dorsal root ganglia (DRG). These neurons send their fibers into the spinal cord where they ascend to medulla and make the first synapse there in nucleus gracilis/cuneatus. The axons of medullar interneurons cross to the contralateral side in medial lemniscus and make the second synapse of the pathway in the ventral posterolateral nucleus (VPL) of the thalamus. This nucleus in turn sends its output to S1. There seems to exist separation between 
different somatosensory modalities all along the somatosensory pathway resulting in separate cortical areas being innervated by different kinds of input (Jones, 1986). Area $3 \mathrm{~b}$ which is located at the posterior wall of the central sulcus (CS) and area 1 which is located immediately posterior to $3 \mathrm{~b}$ at the anterior edge of the postcentral gyrus are the primary recipients of the cutaneous input - the sense of touch. Are 3a which is located deep at the bottom of the central sulcus and area 2 at the posterior edge of the postcentral gyrus are the primary recipients of 'deep' input - from muscles and joints.

Propagation of excitation volley from the peripheral nerve to the cortex is associated with electrical events that can be recorded with electrodes placed on the trunk along the nerve path and on the scalp (Nuwer et al., 1994b). Standards for SSEP recordings were developed mainly for clinical applications and the terminology that developed around the technique was influenced by the particular practices employed by the clinicians. We will, therefore, discuss both the clinical perspective and the broader picture which is sometimes missed by the clinical SSEP recordings. Clinicians usually record SSEPs by placing a recording electrode over S1 contralateral to the stimulation and a reference electrode at the forehead (Nuwer et al., 1994b). Figure 3 shows MNSSEP trace recorded with this montage and scalp voltage maps at several prominent peaks of this trace. The first prominent peak that can be recorded on the scalp is P14 which probably originates from change in volume conductor properties at the passage of excitation volley from the neck to the skull. On the scalp this peak is seen as positive at all electrodes since its negative pole is directed downwards. Thus it appears very small in clinical recording. The effect of processing in the thalamus on SSEP is very weak due to the lack of spatial summation of currents in the thalamus as discussed above. Therefore, the next prominent SSEP peaks are of cortical origin. The N20 peak is the first of these peaks. It is seen as parietal negativity with frontal positivity contralateral to the stimulated hand (and therefore well recorded by the clinical setup). The next peak P25 is seen as central positivity and is not always well separated in the clinical recording. Sometimes it is only seen as small bump at the rising phase of the subsequent larger peak - P30. The scalp pattern of P30 is very similar to that of N20 just with the opposite polarity. It is followed by N35 similar to P25 in scalp pattern but opposite in polarity. These early peaks are followed by a series of later peaks that are not relevant for the present discussion. Although at all these peaks both positive and negative poles can be observed with multichannel 
recording the nomenclature (N- or P- followed by approximate peak latency) follows the polarity seen in the clinical SSEP trace.

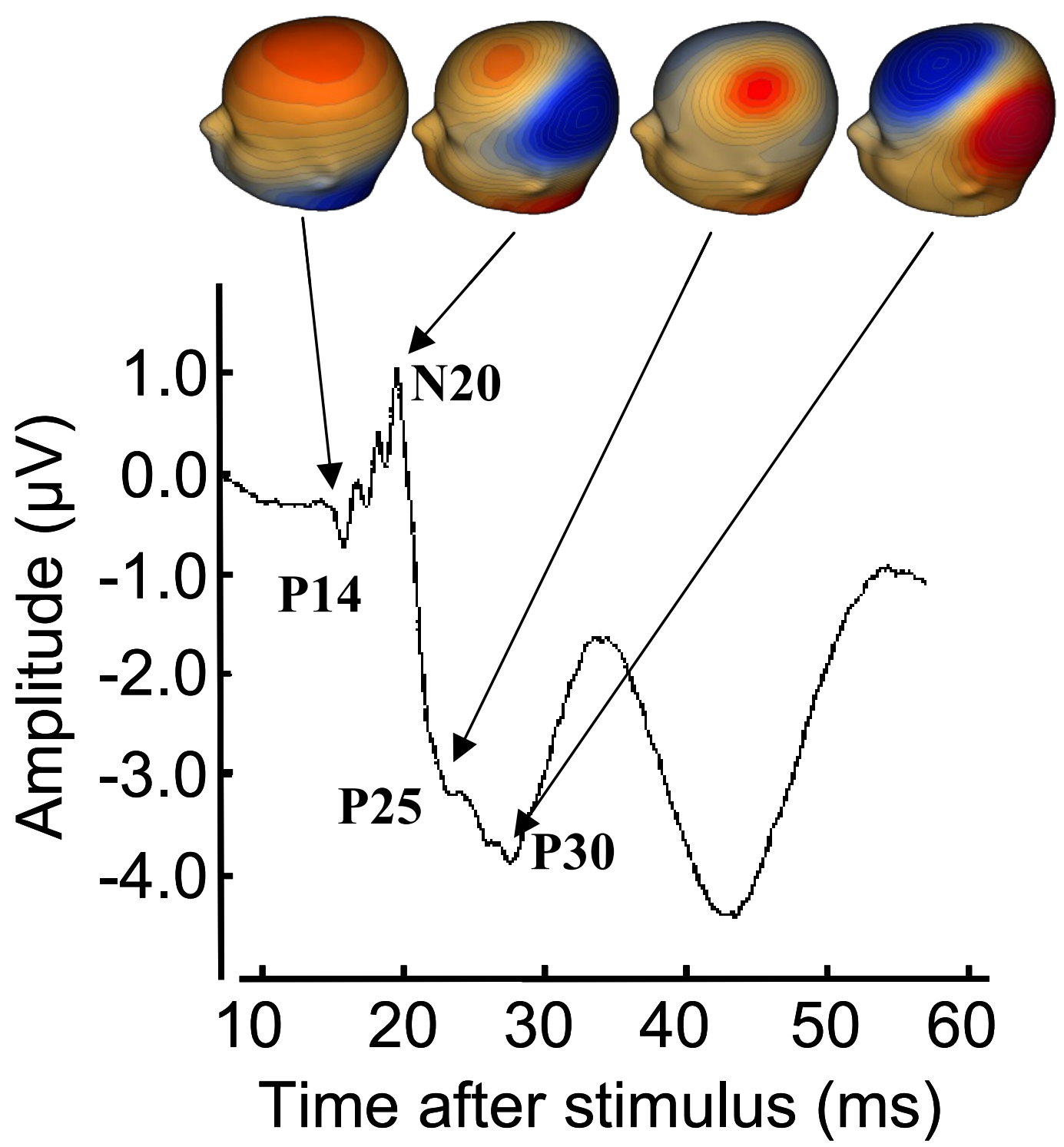

Figure 3. MN-SSEP waveform recorded with clinical recording setup. Above - scalp potential maps at prominent peaks of the MN-SSEP.

Figure 4 shows the presently accepted model that accounts for the patterns described above (Allison et al., 1991). According to this model the N20-P30 complex is generated by area $3 \mathrm{~b}$ and P25-N35 complex by area 1 of the somatosensory cortex. Although both areas are separated from the peripheral nerve by the same number of synapses, the $5 \mathrm{~ms}$ delay between them can be explained by the fact that thalamic fibers projecting to area $3 \mathrm{~b}$ are larger (presumably with correspondingly higher conduction velocities) than those projecting to area 1. Area 3 a may also be involved in the generation of early SSEP. Its contribution can be assessed by stimulating the 
thenar branch of the median nerve in the palm of the hand which is a purely muscle nerve containing the majority of muscle fascicles present in the median nerve at the wrist. When this nerve is stimulated, the amplitude of cortical SSEPs is less than $15 \%$ of the amplitude of median nerve SSEPs and therefore the contribution of area $3 \mathrm{a}$ exists but is probably small.

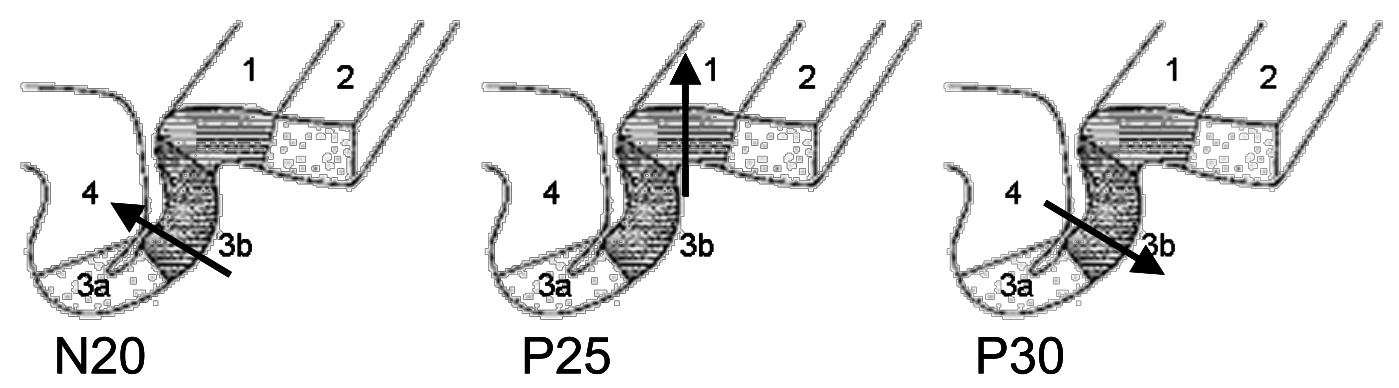

Figure 4. Tangential+somatosensory radial model of MN-SSEP generation (Allison et al., 1991). The arrows indicate direction of current flow. According to the model $\mathbf{N 2 0}$ is generated by thalamic activation of the cell body and basal dendrites of pyramidal neurons of cortical layer 4 (L4) in Brodmann area $3 \mathrm{~b}, \mathrm{P25}$ is generated by a similar albeit slightly delayed process in area 1 and P30 originates again in $\mathrm{L} 4$ pyramidal neurons of area $3 \mathrm{~b}$ when their apical dendrites are activated as a result of processing in recurrent cortical circuits.

\subsubsection{Integration of EEG with TMS}

Since TMS has both immediate and long-lasting effects on the cortex it would be natural to attempt to characterize these effects using the available recording and imaging methods. A few such attempts have indeed been carried out and many more are currently under way. The principal tools are functional magnetic resonance imaging (fMRI) (Bohning et al., 1999; Baudewig et al., 2001; Bestmann et al., 2003; Kemna and Gembris, 2003), positrom emission tomography (PET) (Fox et al., 1997; Paus et al., 2001b; Fox et al., 2005), electroencephalography (EEG) (Ilmoniemi et al., 1997; Ilmoniemi et al., 1999; Boutros et al., 2000; Jing et al., 2001a; Komssi et al., 2002; Strens et al., 2002) and magnetoencephalography (MEG) (Ruohonen et al., 1996). The present discussion will focus on EEG since this is the recording technique used in the studies presented below. However, many of the technical problems and limitations are relevant for the other techniques as well.

EEG can be combined with TMS in two ways one of which is rather straightforward and the other is more technically difficult. When the long-lasting effects of TMS are of interest, EEG can be used as one of the methods for studying the outcome of TMS 
application. This would involve recording the EEG of the subject before and after stimulation with TMS and analyzing various EEG parameters to compare between these two conditions and determine whether TMS caused any changes. The EEG recording in this case does not involve any special technical problems, since there is no interaction between TMS and the recording system. Such studies have indeed been performed aimed mainly at examining the effects of rTMS (Jing and Takigawa, 2000; Jing et al., 2001b; Oliviero et al., 2003).

The second possibility of combining EEG with TMS is to record the transient neural responses to TMS with an EEG system. In this paradigm EEG complements TMS in order to create a tool for measuring functional neural connectivity. Conceptually, the role of EEG in the setup is similar to the role of EMG in the MEP measurements. However, EMG is constrained to measuring connectivity in the motor system, primarily in the spinal cord and the peripheral nerves. EEG does not have such constraints and can measure cortical responses at any location to TMS stimuli at any other location. This opens a way for developing a novel diagnostic and research tool that makes it possible to build maps of functional connections between various cortical regions and studying the dynamics of such maps under different experimental conditions. The major obstacle for this research program is artifacts introduced by TMS into the EEG recordings (Virtanen et al., 1999). Magnetic field fluctuation created by TMS induces electrical currents not only in the brain but also in the EEG electrodes close to the coil. The magnitude of these currents is such that the EEG amplifiers are saturated and cannot record the electrical activity of the brain for some period following TMS. The duration of this period depends on the characteristics of the amplifiers, the pulse magnitude and the distance of the electrodes from the coil. Even relatively short saturation epochs comprise a serious problem since the responses most valuable for the functional connectivity mapping described above are the short latency ones. A few tens of milliseconds after the delivery of a TMS stimulus, extensive cortical regions will be activated and the measured response will not be specific to a particular coil location.

Additional problem is that TMS evokes responses in the brain not only by stimulating it magnetically but also via the conventional sensory channels. At the time of TMS discharge loud click is elicited that evokes auditory responses locked to the stimulus and thus not eliminated by averaging. Moreover, TMS stimulates albeit weakly the 
somatosensory fibers in the skin and in some cases causes slight twitches in head muscles. Thus the ERP produced by averaging many responses to TMS is a mixture of the magnetically evoked responses with auditory and somatosensory components and separating the three is a nontrivial task (Tiitinen et al., 1999). The best approach is to try to reduce the other components as much as possible. To reduce the auditory component subjects wear earplugs and/or headphones with masking noise. However, click sounds can also be conducted by the bone and this effect is much harder to block.

There are several possible approaches for dealing with the problem of magnetic artifacts of TMS. One approach suggested by Virtanen et al. (Virtanen et al., 1999) is using a cut-off circuit that disconnects the electrodes from the amplifiers shortly before the TMS pulse and reconnects them as rapidly as possible after the pulse. This idea was developed over several years by a research group from the Biomag Lab at the University of Helsinki. The first publication using the system (Ilmoniemi et al., 1997) was very promising. By combining EEG, TMS and minimum norm source localization method (see below) it demonstrated propagation of excitation from the stimulated M1 to its contralateral homologue within about $22 \mathrm{~ms}$ after the stimulus. Since 1997 several more studies have been published by this group examining the effect of stimulating with TMS at different intensities at M1 (Komssi et al., 2002; Komssi et al., 2004) and prefrontal cortex (Kahkonen et al., 2004; Kahkonen et al., 2005b). In the recent years a commercial company was established based on this technology and additional research groups are now involved in research combining TMS and EEG using the commercial version of the system. Massimini et al. showed that the EEG responses to TMS are greatly reduced during deep stages of sleep (Massimini et al., 2005a) and that there are difference in responses between schizophrenic patients and normal controls (Massimini et al., 2005b). Esser et al. combined examining both immediate and long-term effects of TMS by showing that EEG responses to TMS at M1 are increased by $5 \mathrm{~Hz}$ rTMS at the same location (Esser et al., 2006). The drawback of the cutoff circuit solution is that the reconnection necessarily takes time during which there is no recording at all. This time varies in different publications from $2 \mathrm{~ms}$ (Ilmoniemi et al., 1997) to as long as $20 \mathrm{~ms}$ (Paus et al., 2001a). In addition, the cut-off circuit itself may introduce artifacts into the recordings. Finally, as will be shown in the present thesis, the cut-off circuit by itself does not remove the artifact completely. 
Another approach that can be explored is using an EEG system with a high dynamic range that will not be saturated by TMS artifacts. The commercial EEG systems that came on the market in the recent years incorporate analog to digital conversion at resolution of 16 or 24 bits. These devices are capable of recording the TMS artifact without saturation and resulting data loss. However, it is still necessary to remove the artifact whose amplitude exceeds by orders of magnitude the amplitude of the physiological EEG response while preserving the physiological component. Thut et al. (Thut et al., 2005) developed an approach that is applicable when TMS is applied while the subject responds to a sensory stimulus and the researchers are interested in the effect of TMS on the sensory ERP rather than in the response to TMS itself. The approach is based on recording responses to TMS with and without the sensory stimulus and subtracting the two. The advantage of this method is that it removes all the contaminants associated with TMS and not just the magnetic artifact. However, the method is unsuitable for examining the responses to TMS itself.

Finally, for some applications only the late responses to TMS not contaminated by TMS are of interest and then the artifact can be just ignored (Bender et al., 2005; Fuggetta et al., 2005).

\subsubsection{EEG oscillations in the sensorimotor system}

The most prominent feature seen in the raw EEG trace is not the event locked responses that can only be revealed by averaging, but rather rhythmic activity at a range of characteristic frequencies (Cooper et al., 1980). The exact frequencies of these rhythmic oscillations differ between subjects and between different states in the same subject. A popular approach accepted also in clinical practice is dividing the range of EEG frequencies into several bands as follows (Cooper et al., 1980): 
Less than $4 \mathrm{~Hz}$ : delta

4 to less than $8 \mathrm{~Hz}$ : theta

8 to $13 \mathrm{~Hz}$ inclusive: alpha

More than $13 \mathrm{~Hz}$ : beta

26-80 Hz: gamma

The slower waves (delta and theta) appear in adults primarily in pathological conditions and during deep stages of sleep. Activity at the alpha range is generated by several distinct sources. The most known is the visual system where alpha activity is characteristic of a relaxed, alert state of consciousness especially with the eyes closed. Low amplitude beta is often associated with active, busy or anxious thinking and active concentration. Gamma rhythms appear to be involved in higher mental activity, including perception, problem solving, fear, and consciousness. The ongoing rhythmic cortical activity is so abundant that some authors claim that ERPs originate not in event-related activation of neural assemblies distinct from background dynamics, but rather produced by phase resetting of ongoing oscillatory activity (Makeig et al., 2002). This view has been challenged by several subsequent studies (Fell et al., 2004; Shah et al., 2004) and the present consensus is that both phenomena take place with possibly different functional roles (David et al., 2006).

In the sensorimotor system rhythmic activity is present at all levels: the muscles and the spinal cord (Mima and Hallett, 1999; Kilner et al., 2002), the tightly interconnected M1 and S1 (Salmelin et al., 1995), higher motor areas (Lee, 2004) and the subcortical structures associated with the motor system - the basal ganglia (Brown and Williams, 2005) and the cerebellum (Gross et al., 2002). Abnormal oscillatory activity apparently plays a crucial role in several diseases of the motor system, most notably in Parkinson's disease (Brown and Williams, 2005; Schnitzler and Gross, 2005).

Planning and execution of movements is associated with characteristic patterns of alterations in the oscillatory activity in the sensorimotor loop. In scalp EEG the pattern consists of event related desyncrhonization (ERD) before and during the execution of movement and event related synchronization (ERS) following the movement (Leocani et al., 1997; Pfurtscheller and Lopes da Silva, 1999). These 
terms, although accepted in the literature, are somewhat misleading since they describe changes in the spectral power of the EEG above a single area rather than changes in synchronization between different brain regions. Similar to the ERP, ERS/ERD is associated with characteristic scalp patterns that depend on the underlying cortical generators. Since information about the current direction is lost when computing power spectra, these patterns are not expected to be dipolar. Figure 5 shows the time course and scalp maps of ERD and ERS phenomena associated with right hand movement. It can be seen that the ERD starts around $2 \mathrm{sec}$ before and continues up to about $2 \mathrm{sec}$ after the onset of movement. It is then followed by ERS an increase in power above the pre-movement baseline level. ERS and ERD are observed in alpha and in the beta frequency range. However, the time-course and other properties of the phenomena in the two frequency bands are not identical and support the view that alpha and beta oscillations affected by movement originate in anatomically and functionally distinct sources. Salmelin et al. (Salmelin et al., 1995) showed using MEG recordings that movement related beta oscillations originate in the precentral gyrus and are topographically organized with respect to the body part involved. Conversely the alpha oscillations originate from the postcentral areas and seem to come from the hand representation independently of the nature of the performed movement. Further evidence for closer relation of the activity in the beta frequency band to the motor system is the existence of cortico-motor coherence (CMC) (Mima and Hallett, 1999; Mima et al., 2000). Coherence as such is a measure of relatedness between two signals, ranging between zero and one and expressed as a function of frequency. Coherence of zero at all frequencies indicates that the signals are not correlated and coherence of one at all frequencies indicates that the signals are related by a linear time-invariant transformation (Cooper et al., 1980). Existence of coherence that is significantly different from zero at a given frequency range indicates that there is a common component in the two signals at these frequencies. The reason for this might be either that one of the generators involved drives the other or that both generators receive common input from a third source. EMG activity during tonic muscle contraction was shown to be coherent in the beta range with the EEG recorded above the contralateral motor area (Mima et al., 2000). By studying patients with early brain lesions in whom the motor representation of both hands was located in one hemisphere while the somatosensory representation was bilateral Gerloff et al. (Gerloff et al., 2006) showed that the coherence follows the motor representation 
rather than the sensory one. This does not prove conclusively, however, that $\mathrm{S} 1$ is not involved in the circuit driving the muscles in healthy subjects.
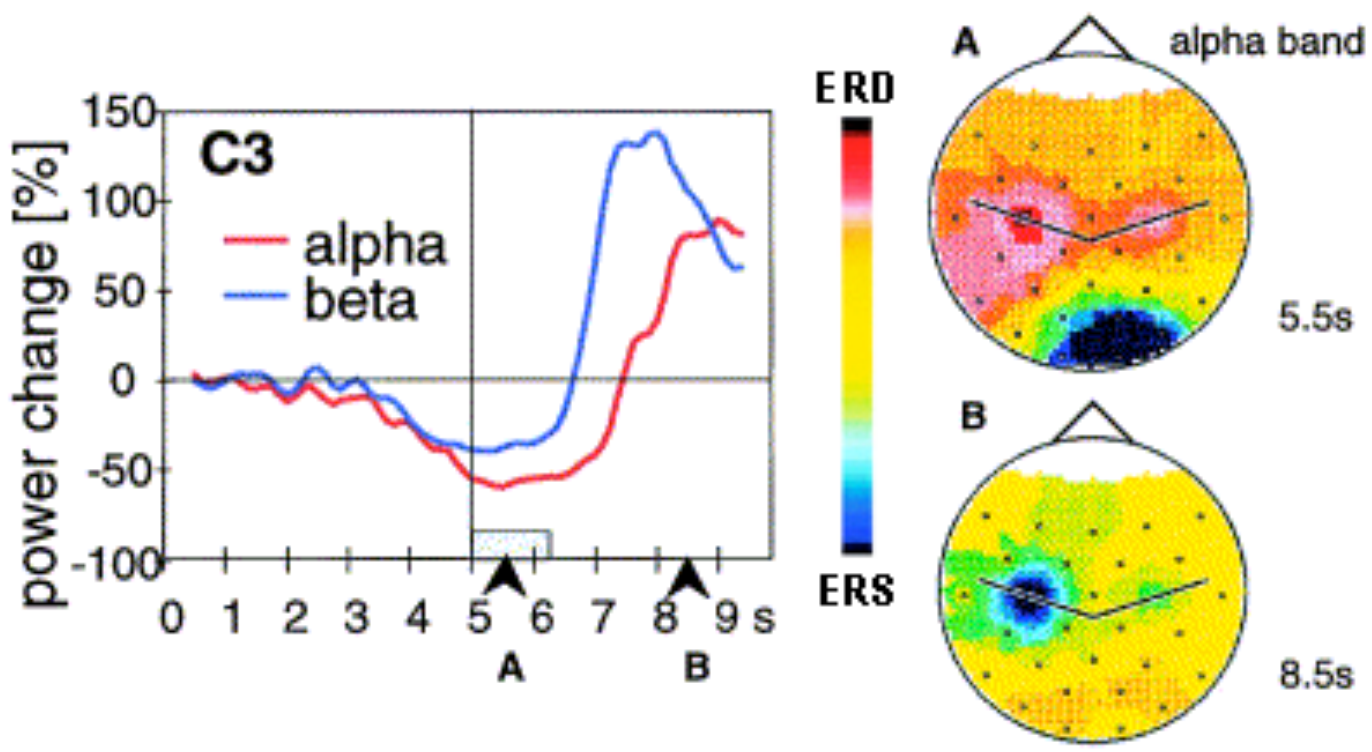

Figure 5. Time course and scalp pattern of ERD/ERS associated with hand movement. Grand average $(n=9)$ ERD curves calculated in the alpha and beta bands in a right hand movement task (left side). Grand average maps calculated for a $125 \mathrm{~ms}$ interval during movement (A) and after movement-offset in the recovery period (B) (right side). The scalp electrode positions are marked. 'Red' indicates power decrease or ERD and 'blue' power increase or ERS. Adapted from (Pfurtscheller and Lopes da Silva, 1999).

Several studies involving recordings of LFP from the subthalamic nucleus (STN, the target of the DBS therapy) and EEG/MEG from the cortex indicate that beta oscillations and increased beta band coherence are "anti-kinetic" i.e. associated with decreased ability to initiate movements, or in other words, with maintenance of the existing motor set (Gilbertson et al., 2005). Conversely, increased oscillations and coherence in the gamma band are "pro-kinetic" i.e. associated with movement initiation (Brown and Williams, 2005) and readiness to respond (Schoffelen et al., 2005). Beta band oscillations in the STN decrease starting about $2 \mathrm{sec}$ prior to movement onset (similar to the time course of the ERD) with an onset latency that is strongly correlated with mean reaction time across patients (Kuhn et al., 2004). A similar phenomenon was also observed during motor imagery without execution of movements (Kuhn et al., 2006). Treatment with Levodopa in Parkinsonian patients increases the magnitude of beta desynchronization in the STN and it is associated with improvement in motor task performance (Doyle et al., 2005). Parkinson's 
disease also affects the ERD seen in the scalp EEG. Defebvre et al. showed that in patients ERD ocurrs later than in healthy controls (Defebvre et al., 1994).

Since movement planning and execution is associated with increased M1 excitability manifested in increased MEP amplitude (Zaaroor et al., 2001, 2003) a positive correlation should exist between the degree of ERD and MEP amplitude. Such correlation was indeed found in M1 ipsilateral to the movement (Rau et al., 2003). No significant change in M1 excitability was associated with post movement ERS.

In summary ERD in the beta band is associated with movement planning and increased motor excitability, whereas increase beta oscillations at all levels of the motor system and increased beta band coherence correlate with maintenance of posture and in pathological cased with rigidity and deficient movement initiation. ERD-ERS sequence around movements can also be seen in the alpha band. However, in this case it is probably generated in the somatosensory system and is related to processing of movement related sensory feedback. 


\subsection{ERP source localization}

\subsubsection{The forward and the inverse problem}

The signal recorded on the scalp by an EEG electrode is a result of summed contributions of multiple sources; among them the brain activity of interest, other brain sources and artifacts both biological and non biological.

In order to simplify the analysis of these contributions it is assumed that their summation is linear. This is a good approximation in the case of EEG.

Under the assumption of linear summation the relationship between the scalp voltage and the activity of the underlying sources can be written as follows:

$$
V_{n \times t}=L_{n \times m} \cdot S_{m \times t}+\mathrm{E}_{n \times t}
$$

$V_{n \times t}$ is the matrix of observed voltages ( $\mathrm{n}$ - number of channels, $\mathrm{t}$ - number of time frames). Each row of $V_{n \times t}$ is a waveform recorded by one of the electrodes and each column is a voltage map on the scalp at a specific time point.

$S_{m \times t}$ is the matrix of source activities, where each row is a source waveform. $\mathrm{m}$ is the number of sources. The units of $\mathrm{S}$ are dipole moment $(n A \cdot m)$.

$E_{n \times t}$ is the noise. At this stage we do not distinguish between brain activity and other sources in $S_{m \times t}$ so some of its rows may represent noise sources as well. The distinction between $S_{m \times t}$ and $E_{n \times t}$ is in the fact that noise sources affecting multiple electrodes (such as eye blinks) will be treated as part of $S_{m \times t}$ whereas noise sources affecting each electrode separately (such as amplifier noise) will be included in $E_{n \times t}$. As we will show below it is possible to estimate the contribution of the former kind of noise sources and separate it from brain activity whereas not much can be said about the latter kind of sources. Therefore $E_{n \times t}$ represents the residual, not explained by our source model. 
$L_{n \times m}$ is called 'lead field matrix' and each of its columns is a 'scalp topography' or 'scalp signature' of the corresponding source. This topography would be observed on the scalp is this source was the only active source contributing to the EEG.

Given the location and orientation of the source in the head and a model of the head including the shape, thickness and conductivity of different tissue layers $L_{n \times m}$ can be computed based on the laws of physics (Mosher et al., 1992). Therefore if the location and orientation of brain sources and their activity $\left(S_{m \times t}\right)$ is known, the EEG signals expected in the absence of noise can be computed precisely. This problem of computing voltage distribution on the scalp given the underlying brain sources is called 'the forward problem of EEG' and in most cases it is quite tractable.

The 'inverse problem' is the problem of computing the underlying current source distribution in the brain based on voltage distribution on the scalp. The inverse problem is usually ill-defined since the number of possible current sources exceeds by far the number of EEG sensors. Therefore, the inverse problem has to be solved under constraints to reach a unique solution.

To solve the inverse problem we have to find $S_{m \times t}$ and $L_{n \times m}$ that can explain the observed $V_{n \times t}$ and this is usually expressed mathematically as minimization of the expression:

$$
\left\|V_{n \times t}-L_{n \times m} \cdot S_{m \times t}\right\|
$$

where $\|\cdot\|$ is the Euclidean norm. A large part (but not all) of the existing EEG source localization methods are particular cases of solving the above equation with different assumptions.

\subsubsection{Overview of existing source localization methods}

The existing approaches of solving the inverse problem can be divided into two large groups. The approaches of the first group place a large number of fixed sources in the brain, capable of representing the activation of any brain region. These sources can either be distributed all over the brain volume on an equally spaced grid, constrained 
to the gray matter or even distributed and oriented in accordance with the individual brain anatomy as measured with anatomical MRI scan. In any case $L_{n \times m}$ is a fixed property of the anatomical brain model and does not depend on the EEG data. Also in order to represent any possible brain activity a large number of sources is necessary which exceeds by far the number of EEG sensors $(m ? n)$. Therefore $L_{n \times m}$ is singular and cannot be inverted. As a result, for any given instantaneous voltage distribution $\mathrm{V}$ and infinite number of source activation vectors $\mathrm{S}$ exist that satisfy $\left\|V-L_{n \times m} \cdot S\right\|=0$ or equivalently $V=L_{n \times m} \cdot S$. In order to select from the infinite number of solutions a particular one an additional criterion has to be introduced. This criterion has to be something that would characterize a 'realistic' biological solution. Mathematically the problem can be formulated as:

$$
\min _{S} S^{T} W S \quad \text { s.t. } \quad V=L S
$$

This problem has a well defined solution:

$$
S^{*}=W^{-1} L^{T}\left[L W^{-1} L^{T}\right]^{+} V
$$

where $[A]^{+}$denotes Moore-Penrose pseudoinverse of matrix A (Pascual-Marqui, 1999). Different methods differ in their definition of matrix W. Hamalainen and Ilmoniemi (Hamalainen and Ilmoniemi, 1994) suggested $W=I_{m \times m}$ (the identity matrix). In this case $S^{T} W S=\|S\|^{2}$ and what is minimized is just the norm of the source activation vector. Thus the name of the method is 'minimum norm solution'. The idea behind it is that solutions which explain the given scalp pattern by minimal amount of cortical activity are more likely to be realistic. Subsequently it was shown that minimum norm solution overestimates superficial sources that have strong scalp signatures and underestimates deep sources. Thus a variant 'weighted minimum norm' was suggested where $w_{i j}=\left\{\begin{array}{cc}\sum_{k=1}^{n} l_{k i}^{2} & i=j \\ 0 & i \neq j\end{array}\right.$. This effectively normalizes each source's activation by the power of its scalp topography. Also other possibilities for $\mathrm{W}$ were suggested that take into account the noise correlations. 
Pascual-Marqui et al. (Pascual Marqui et al., 1994) suggested a different principle for building $\mathrm{W}$, which is a principle of maximal smoothness. W (whose exact definition is too complex to present here and may be found in the original publication) incorporates the Laplacian operator so that the principle of optimization is making the activity making the activity of each source as similar as possibly to the activity of sources located close to it in space. The justification for this approach is the idea that what is seen on the scalp is in any case synchronized activity of large cortical patches. This approach and the software based on it are called 'Low Resolution Electromagnetic Tomography' or LORETA.

In all the cases there is no guarantee that any choice of W no matter how well justified by theoretical considerations will lead to the actual physiological solution. For any of the approaches described above one can think of biologically plausible cases that would violate its assumptions. Therefore, the validation of each approach is empirical - based on computing the sources of evoked potentials whose generators are well known from PET and fMRI studies or intracranial recordings.

Another direction for finding biologically realistic solution for equation (2) is assuming that the brain activity captured by $V_{n \times t}$ is generated by a small number of point sources or dipoles. Thus, $m=n$. On the first glance this assumption seems unrealistic knowing the vast number of functionally distinct brain structures and cortical areas. However, when $V_{n \times t}$ is an averaged event related potential, the activities of sources not locked to the event of interest are averaged out and this greatly reduces the number of contributors. In addition it can be shown by computer simulations that uniformly activated planar cortical sheet with the size of up to $4 \mathrm{~cm}^{2}$ would produce scalp signature practically indistinguishable from the signature of the point source located in the middle of such a sheet (Scherg and Hoechstetter, 2000). This happens due to 'blurring' of the signal by the skull. Therefore, in many cases it is possible to build models with just a few point sources that accounts for most of the observed scalp signal. For such models the problem $\min _{S}\left\|V_{n \times t}-L_{n \times m} \cdot S_{m \times t}\right\|$ given $\mathrm{V}$ and $\mathrm{L}$ is overdetermined and a unique solution exists given by:

$$
S=L^{+} V
$$

where

$$
L^{+}=\left(L^{T} L\right)^{-1} L^{T}
$$


is the Moore-Penrose pseudoinverse we already encountered above (Scherg and Berg, 1996). It is a mathematical property of this solution that:

$$
\sum_{k=1}^{n} l_{i k}^{+} l_{k j}=\delta_{i j}= \begin{cases}1 & i=j \\ 0 & i \neq j\end{cases}
$$

This means that in the absence of noise the solution reconstructs the source activity exactly. However, some amount of noise is always present and more importantly in reality we should not confuse the lead field matrix L originating in our source model from the actual lead field matrix of the corresponding brain sources that is unknown to us. The model L may differ from the reality both because of imprecision in the source model (locations, orientations or even the number of point sources differ from those of real sources) and imprecision of the head model (an idealized model such as 3-layered sphere differs from the actual subject's head both in shape and in conductivity). As a result, in reality the reconstruction of source activities will always be imprecise, especially when source topographies are similar. In such cases noise can be amplified because of the demand that the solution separates these similar topographies. Therefore in practice one prefers to sacrifice the precise solution, which us not precise anyway in favor of controlling the noise and this is done using Tikhonov regularization (Tikhonov and Arsenin, 1977). In this method instead of just minimizing (2) an additional term is added:

$$
S(t)^{*}=\min _{S(t)}\left\{\|V(t)-L \cdot S(t)\|^{2}+\alpha^{2}\|S(t)\|^{2}\right\}
$$

for some suitably chosen 'Tikhonov factor' $\alpha>0$.

$$
S_{\alpha}^{*}=\left(L^{T} L+\alpha^{2} I\right)^{-1} L^{T} \cdot V
$$

An optimal choice for $\alpha$ according to Bayesian approach would be the inverse of the signal to noise ratio (SNR). Thus when there is no noise $\alpha$ can be small and the solution will be close to the ideal case of equation (5).

\subsubsection{Constructing multiple source models}

Until now we discussed how source activities can be computed given a source model of the data which is defined by the lead field matrix $\mathrm{L}$ and the data recorded on the scalp. It still remains to be explained how the source model and the matrix L derived from it are built. 
Let us consider a simplified case when the source model consists of a single equivalent current dipole and therefore $L_{n \times 1}$ is a column vector. $L_{n \times 1}$ can be computed by solving the EEG forward problem given the location and orientation of the dipole. In total there are 5 degrees of freedom ( 3 for location and 2 for orientation). We can now look at the problem of optimizing these 5 parameters to minimize the difference between the actual observed scalp voltage and the prediction of the model.

$$
\min _{L(x, y, z, \theta, \phi)}\left\{\left\|V-L\left(L^{T} L+\alpha^{2} I\right)^{-1} L^{T} \cdot V\right\|^{2}\right\}
$$

This multivariate optimization problem can be treated with modern optimization tools in order to find numerically the optimal solution. The algorithm implemented in the software used for data analysis in the present thesis is the simplex algorithm for nonlinear optimization suggested by Nedler and Mead (Nelder and Mead, 1965). Since the optimization algorithm is not limited to five parameters it is also possibly to simultaneously fit more than one dipole. However, the more free parameters are optimized the more complex is the resulting cost function and the higher the chance to for the optimization process to get stuck in a local minimum. Therefore, while fitting a single dipole usually leads to a clear result, fitting a set of dipoles is rarely biologically meaningful. More complex but biologically meaningful models can be built by introducing dependencies between several dipoles to keep the number of parameters small. An example of this principle is the 'regional source' (Scherg and Von Cramon, 1986) which consists of 3 orthogonal dipoles sharing the same location. This complex of 3 dipoles can fit any activity originating at a particular location. The goodness of fit does not change by reorienting the source. Thus, it can be described just by the three location parameters and its fitting is mathematically even simpler and more stable in the presence of noise than fitting a dipole. A useful technique is fitting a regional source and then reorienting it so that its components would be biologically meaningful - for instance fitting the orientation of one of the components to the scalp voltage topography at an ERP peak. Then the source waveforms of the 3 dipoles can be examined and if one or two of them do not contain significant activity, they can be deleted. So the final result of the process might be again a single dipole, but its location will be more reliable, especially in the presence of noise, than the one that would be obtained by fitting a single dipole in the first place. Another meaningful construct is a complex of two regional sources whose locations are constrained to be 
symmetric with respect to the midline. The number of parameters to be fitted is still three. This complex of two dipoles is well suited for fitting synchronous bilateral activity such as auditory evoked responses.

The above discussion clarifies how activity originating from a small brain region or two symmetric small brain regions can be modeled. A problem arises when activity is generated by sources distributed in more complex way. Trying to fit a single dipole or regional source to activity generated by several distant sources will result in incorrect model. The model source will be placed in a location where it can simultaneously explain the activity of several generators and this location will probably not correspond to any of these generators.

Sequential fitting approach is a way to bypass this problem. It is based on the assumption that at least at some stages during the response the activity is dominated by a single source or two homologous sources in the two hemispheres. For primary sensory responses this is usually true at the very early stages when primary sensory areas are activated. For later processing stages this can also happen when prominent ERP peaks are observed. If a segment like this can be found, the activity there can be modeled as described above and the locations of the model sources will reflect the location of the actual brain sources correctly. There is still a doubt even in this simple case whether to use a single regional source or two symmetric regional sources. The decision should be based on prior knowledge about whether the activity can be bilateral and on examining the scalp maps. In complex cases both alternatives can be tested and compared.

After some part of the activity is modeled, the residual can be examined given by:

$$
R=V-L\left(L^{T} L+\alpha^{2} I\right)^{-1} L^{T} \cdot V
$$

The residual approximates the activity not explained by the model at this stage. In this residual activity one can again look for segments where only a single source is dominant (in addition to the already modeled sources) and fit a new source to these segments while keeping the previously fitted sources fixed. It should be stressed that the new source is not fitted to the residual that is present after fitting the previous sources. Rather, the residual of the combined model is minimized by fitting the new source. Thus, the data explained by the previously fitted sources changes during the 
fitting process of the new source. The process is repeated until the residual is just random noise. In practice, this can be seen by eye as absence of clear peaks in the waveforms and absence of clear dipolar patterns in the scalp maps of the residual.

Since the model activity is computed at every stage in a way that the residual will be minimal, the residual does not contain any activity that can be accounted for by the existing model sources. In reality, however, at least part of this activity may come from other sources, which are not yet in the model. In fact, without knowing the correct locations of all the sources, their activities cannot be separated correctly. On the other hand, when a source is fitted not to its actual well separated activity, but to residual not explainable by other sources, its location might be determined incorrectly. This 'chicken and egg' problem is partially remedied due to the fact that the fitting process is iterative and the source activities as well as the residual are recomputed at each step. Therefore, if at the beginning of the fitting process the source starts moving toward the correct position, it will separate a larger part of the actual activity of the underlying brain source correctly, and will be positioned better at the next step. Nevertheless, the sequential fitting approach works well when the actual brain source configuration is relatively simple in space and time segments where the residual activity is dominated by a single source can be found at each stage of the fitting process.

An alternative to sequential fitting that can also be combined with it is 'seeding'. Seeding is useful when the locations of the sources are known and the interest is in their activities. This situation arises, for instance, in studies combining EEG with fMRI. fMRI is a method with spatial resolution far exceeding that of EEG. However, its temporal resolution is poor because it measures changes in blood oxygenation rather than neuronal activity. fMRI and EEG can be performed in the same experiment either simultaneously or in separate sessions. Then regional sources are placed in locations where significant activity is detected in fMRI, and reoriented if necessary to produce clear waveforms. This approach can work well when fMRI and EEG indeed observe the same brain sources. This, however, is far from being the rule. Nunez and Silberstein (Nunez and Silberstein, 2000) discuss this issue in detail and their conclusion is that because of the different nature of signals detected by EEG and fMRI in many cases changes in neural activity will affect one and not the other imaging modality. 
Seeding can also be used when the sources involved in generating the evoked response in question are known from previous studies. The basic auditory evoked potentials (AEP), somatosensory evoked potentials (SSEP) and visual evoked potentials (VEP) have already been modeled (Scherg and Von Cramon, 1986; Buchner et al., 1995; Di Russo et al., 2002) and the results of this modeling were shown to be consistent with fMRI and intracranial recordings. Therefore, for new studies of these potentials where only source activity is of interest standard models can be used. Scherg et al. (Scherg et al., 2002) propose the 'source montage' approach when source activity is computed directly from the data using a standard source model. Separate models are presently available for AEP, SSEP and VEP. These models contain in addition to specific sources for the respective evoked potentials also non specific regional sources distributed at other key locations in the brain to prevent the specific sources from picking unrelated activity as discussed above. As already mentioned, combining different approaches is also possible. For instance, while examining responses to speech one can start with a standard model for primary auditory cortex activity and then try to model the residual using sequential fitting.

\subsubsection{Artifact correction using multiple source models}

A specific case which is of interest in the present thesis is the use of discrete multiple source modeling for correcting artifacts. There are artifacts present in practically any EEG recording, such as eye movements, blinks and muscle artifacts and this thesis deals specifically with artifacts generated by transcranial magnetic stimulation (TMS). Under the assumption of linear summation which is the basis of all the methods discussed until now, artifacts can be presented as part of the sources in matrix S. The main differences between modeling artifacts and brain sources is that we do not expect artifacts to be well modeled by equivalent dipoles and we are not interested in characterizing the artifact sources, but only in separating between artifact and the brain activity. Under these conditions it is not necessary to have a physical model of artifact generation, but it is enough to somehow estimate a column in matrix $\mathrm{L}$ representing the effect of the artifact on the electrodes. This can be done either by taking scalp topography at a timeframe dominated by the artifact or by performing principal component analysis (PCA) on an artifact dominated time segment. PCA is a linear transformation that chooses a new coordinate system for the data set such that 
the greatest variance is accounted for by the first axis (called also the first principal component). Then the second axis orthogonal to the first is chosen to account for the greatest remaining variance and so on. The idea is that in many cases although the dimensionality of the raw data is high (in the case of EEG dictated by the number of electrodes) the number of underlying sources is low. Equation (1) describes such a case when $m=n$ and the noise $\mathrm{E}$ is negligible. In this case only the $m$ first principal components will account for high variance. PCA is not a good method for extracting biological sources because they are rarely orthogonal. However, in the case of artifact we are only interested in finding a way to represent the artifact topography by a small number of components and PCA is a mathematically optimal way of doing this. An artifact component derived with one of the two methods described above interacts with other components in the same way as biological components that we already discussed. This means that when scalp topographies of biological sources are correlated with artifact topographies the artifact can only be well isolated in the presence of a good model for non-artifactual activity. If such a model does not exist, we find ourselves once again in 'chicken and egg' situation where we need a good model for the data to subtract the artifact but such a model cannot be built before the artifact is subtracted. The solution is to use standard source models described above. Since the standard models do not describe the biological activity perfectly, the separation will not be perfect, but it can be good enough for some applications. It is also possible to work iteratively - subtracting the artifact using a standard model, build a source model based on the artifact corrected data and then use the new model instead of the standard model to separate the biological activity from the artifact in the raw data. In the absence of a perfect model for the biological component of the data (which is always the case) there will be distortions and some of the biological activity will be projected to the artifact waveforms whereas some remains of the artifact will be projected to waveforms representing biological sources. If an artifact cannot be represented by linearly combining small number of topographies it cannot be corrected with the method described above without significantly distorting the data because some of topographies representing it will necessarily be correlated with topographies of biological sources. This problem is especially severe when the number of the electrodes is low. But in any case, artifact correction should not be done blindly and it is sometimes preferable to exclude part of the data containing large artifacts from the analysis to minimize the distortions in the remaining data. 


\subsection{Spectral analysis}

Although event locked averaging reveals physiologically meaningful spatio-temporal patterns in the EEG, it is far from being able to extract all such patterns. For instance, changes in the cortical oscillatory activity that are not phase-locked to the averaging trigger are averaged out and do not affect the ERP (David et al., 2006). Furthermore, in many cases it is of interest to compare EEG activity between two functional states of the brain (for instance between wakefulness and drowsiness) with no averaging triggers available. In such cases Fourier analysis can be applied to the EEG data to examine its features in the frequency domain. In the present thesis spectral analysis was used to compare ongoing cortical activity preceding a TMS pulse between two conditions defined based on the amplitude of MEP measured after the pulse. Since the pulses were delivered at random times, there was no reason to expect any timedomain ERP pattern in the activity before the stimulus. Thus the only sensible way of analysis was to examine the EEG spectra.

The exact definition of the spectrum of a stochastic process is beyond the scope of the present discussion and can be found for instance in (Bendat and Piersol, 2000). It would suffice to say that a simple estimate of the spectrum of a time series $\left\{x_{t} \mid t=n \Delta t, n=1,2, \mathrm{~K} N\right\}$ is given by squaring its Discrete Fourier Transform (DFT) defined as:

$$
\ell(f)=\sum_{n=1}^{N} x_{t} e^{-2 \pi i f n \Delta t}
$$

where $\Delta t$ is the sampling interval. This estimate, however, suffers from two problems. First, it only converges to the actual spectrum for infinite data length. If the time series is finite the transformation described in Equation (12) is equivalent to multiplying the hypothetical infinite time series with a boxcar taper before applying the Fourier transform. In frequency domain this is equivalent to convolving the actual spectrum with Fourier transform of the boxcar taper, a function called sinc.

Convolution operation causes 'spectral leakage' meaning that the estimate of spectrum for some frequency $f$ if affected by the actual values of the spectrum at other frequencies which may be quite distant from $f$. This problem can be partially remedied by multiplying the data by a taper with better spectral properties before 
performing the Fourier transform. In order to minimize the resulting distortion the Fourier transform of the taper should be as similar to the Dirac delta function as possible. From this point of view the boxcar taper is far from being the optimal choice. Secondly, even if the data length is infinite, $|\ell(p f)|^{2}$ will not converge to the spectrum of the random process but rather to the spectrum of the particular realization of it that was examined. In statistical terms it means that $|\ell(f f)|^{2}$ is an inconsistent estimator of the spectrum (Bendat and Piersol, 2000). This problem is usually solved by performing averaging either in the time domain (i.e. dividing the time series into several segments, computing their spectra and averaging) or in the frequency domain (i.e. dividing the frequency axis into bins and averaging within each bin). This produces a better estimator because for a stationary process both estimators derived from non-overlapping time segments and estimators derived from neighboring frequencies are independent (Mitra and Pesaran, 1999).

Thomson (Thomson, 1982) suggested an improved method for spectral estimation that elegantly solves both problems in an integrated way. His suggestion was based on previous research of Slepian and Pollak (Slepian and Pollak, 1961) aimed at developing tapers of length $\mathrm{N}$ whose spectrum approximates the delta function. Such tapers should have as much energy as possible concentrated in a narrow frequency range $[-\mathrm{W} \mathrm{W}]$ where the parameter $\mathrm{W}$ determines the amount of smoothing in the frequency domain. This requirement can be mathematically formulated as an optimization problem whose solutions are a set of orthogonal eigenvectors of a matrix. Remarkably, the first $2 \mathrm{NW}$ of these eigenvectors have eigenvalues close to 1 while the rest have eigenvalues close to zero. Since the Slepian tapers are orthogonal, using each of them leads to an estimator which is independent of the estimators based on the other tapers. These independent estimators can be averaged with the result being a consistent estimator for the spectrum of the stochastic process generating the data. It is called "the multitaper spectral estimator" $\left(S_{M T}\right)$.

$$
S_{M T}(f)=\frac{1}{K} \sum_{k=1}^{K}\left|\mathscr{V}_{k} 0(f)\right|^{2}
$$

where 


$$
\ell_{k} 0(f)=\sum_{n=1}^{N} w_{t}(k) x_{t} e^{-2 \pi i f n \Delta t}
$$

and $w_{t}(k)$ is the $\mathrm{k}$-th taper.

The usual strategy for the multitaper spectral analysis is to select the desired analysis half-bandwidth $\mathrm{W}$ to be a small multiple of the frequency $1 / \mathrm{N}$, and then use the leading $\mathrm{K}=2 \mathrm{NW}-1$ tapers. An example of such a series of Slepian tapers can be seen in Figure 6. Less than $2 \mathrm{NW}$ of the sequences are typically taken, since the last few of these have progressively worsening spectral concentration properties. On the other hand, to produce a better estimator and reduce noise $\mathrm{K}$ should be increased. This can also be done by increasing $\mathrm{N}$ or $\mathrm{W}$ at the expense of resolution in time and in frequency respectively. No simple procedure can be given for these choices, because the choice really depends on the data set at hand, and is best made iteratively by visual inspection and some degree of trial and error (Mitra and Pesaran, 1999).

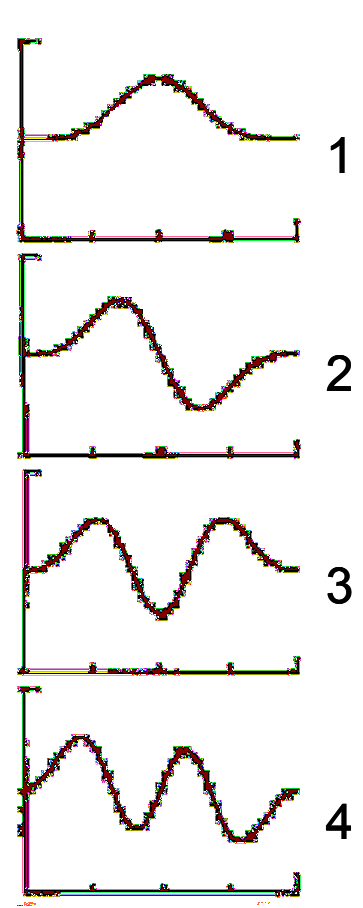

Time

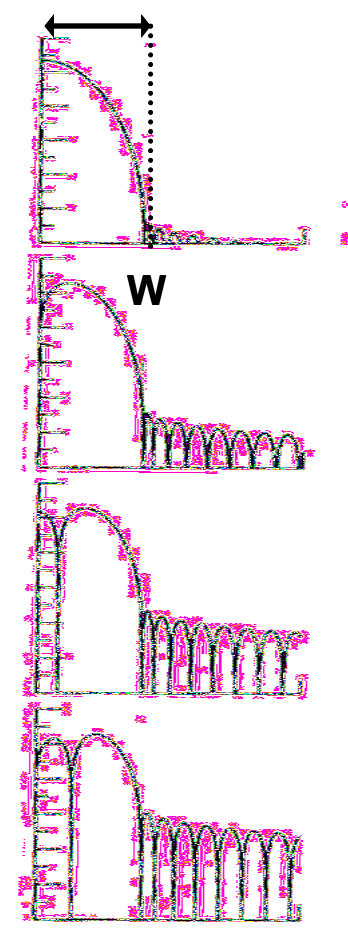

Frequency

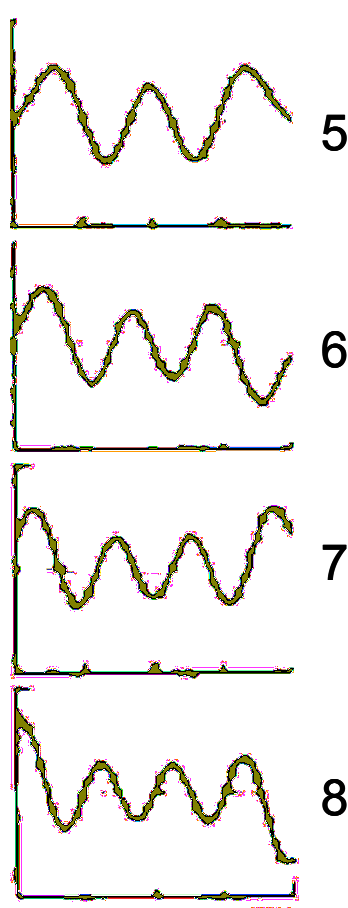

Time

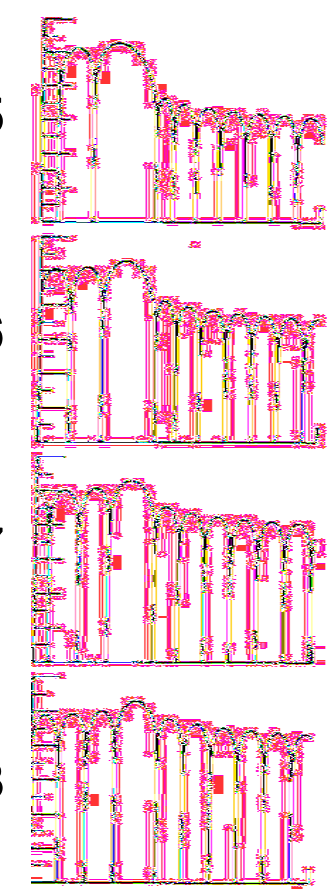

Frequency

Figure 6. Waveforms and spectra of a series of tapers used for multitaper analysis. The spectral concentration properties worsen for the later tapers in the series.

The multitaper analysis is especially suitable for EEG and other types of neural data for several reasons. First, EEG data is inherently non-stationary and in order to 
approximate the stationarity assumption, on which the spectral analysis is based, the data should be divided into short time windows. This is also necessary for timefrequency analysis in order to follow the evolution of spectral properties of the data across time. Using short time-windows worsens the leakage problem and thus some kind of tapering is absolutely necessary. Slepian tapers are optimal in terms of spectral concentration and make it possible to balance the resolution and the variance of the estimator. Another property of neural signals is that their spectra are always smooth in the frequency domain and thus it is natural to estimate the value of the spectrum for a particular frequency based on the neighboring frequencies as the multitaper analysis does. Unlike standard averaging methods in time and frequency that are based on the mean of estimators already distorted by the leakage, averaging of Equation (13) is effectively over estimators derived from the 'ideal' spectrum of the stochastic process and thus has better properties. On the other hand multitaper analysis is still a non-parametric method not making any assumptions about the character of the estimated spectrum. Thus it is more suitable for biological data with complex spectra than parametric autoregressive methods that can adequately model only spectra with a small number of peaks (Thomson, 1982).

Mitra and Pesaran (Mitra and Pesaran, 1999) make a detailed argument for the multitaper analysis as the method of choice for a wide variety of applications in neural data analysis. In the present study we used the implementation of the multitaper analysis which is part of the Fieldtrip toolbox (see Methods). 


\subsection{Statistical analysis}

\subsubsection{General considerations}

Analyzing multi-channel EEG/ERP data presents a considerable methodological challenge. The data is inherently multidimensional. Moreover, in the context of neural data analysis there are several ways to define what constitutes a dimension. From the point of view of random signal processing even a signal recorded from a single electrode is multidimensional because the voltage at each time point can have different statistical characteristics if examined across multiple trials. From the point of view of computer processing a dimension is some parameter according to which data can be classified and ordered. But there is also a physical/intuitive interpretation of dimension which is some parameter the data represents in the physical world. In the following discussion we will adhere to the latter definition, unless specifically stated otherwise. In the simplest case of raw EEG/ERP recording one has to deal with the spatial dimension, namely the identity and thus location of the recording channel, and the temporal dimension - the time when a particular voltage value was recorded in a given channel. Yet, even in this simplest case the channel identity actually represents 2D spatial information because the electrodes sample the two-dimensional voltage distribution on the scalp. This must be taken into account in order to present the relations between the different channels in a biologically meaningful way. The picture gets more complicated when spectral analysis is applied. In the case of spatiotemporal decomposition such as wavelet decomposition or Fourier analysis with multiple time windows the frequency dimension is added to the time dimension, making the data $3 \mathrm{D}$ (Channel $\times$ Time $\times$ Frequency) in the data processing sense but actually $4 \mathrm{D}$ in the physical sense. Finally, when source localization is performed by an underdetermined method estimating current source density in the brain, the data may become 5D $(\mathrm{X} \times$ $\mathrm{Y} \times \mathrm{Z} \times$ Time $\times$ Frequency) and when looking at multiple subjects, multiple experimental conditions and multiple trials in each subject we easily reach $8 \mathrm{D}$. Only in recent years with the development of powerful computers it has become feasible to even start thinking about handling realistic datasets of this dimension. But computers do not solve all problems, first because they are sometimes not powerful enough and more importantly because the human experimenter cannot examine the results of detailed analysis of all the available data and his mind is unable to grasp and interpret such results. 
Two traditional methods for dealing with complexity are selection and averaging. For instance, when studying the potentials evoked by stimulation of the right median nerve, a researcher may look only at the voltage difference between an electrode overlying the primary somatosensory cortex, such as CP3, and a reference electrode on the forehead. This will eliminate the spatial complexity of the data. Since responses to individual stimuli are dominated by noise, one can average the responses over trials to produce the somatosensory ERP. Moreover, instead of examining the potential at every time point, the researcher may decide that he is interested in the amplitude of a particular voltage deflection in the average ERP that is known to occur around a specific time in healthy subjects, called N20 peak. This eliminates the time dimension. Alternatively, one can look at the average ERP amplitude or average power between 18 and $20 \mathrm{~ms}$. Thus, a single data point per condition/subject combination can be extracted. This set of data points can be handled using traditional statistical methods, such as analysis of variance (ANOVA) and t-test. Similarly, when analyzing spectral data one can average over particular frequencies of interest and look at 'power at the beta band above the motor cortex'. This approach cannot be criticized, since for many years it was used out of necessity rather than as a matter of choice. Its main disadvantage is, however, the need to produce educated guesses for what to select and over what to average. This can lead to looking under the streetlight and completely missing physiologically important phenomena if they do not occur when and where expected. In addition, it inevitably leads to a way of thinking dominated by measurement-specific rather than biological terms such as 'P300 amplitude' or 'increase in alpha power at occipital channels'.

In order to better capture the physiological reality in its entirety, it is desirable to abandon this approach, reduce the role of subjective selection and averaging in the data analysis and instead work in a data-driven rather than a hypothesis-driven way. This, however, brings us back to the problem of complexity and high dimensionality of the data. A set of statistical tools developed in recent years (Nichols and Holmes, 2002; Maris, 2004) makes it possible to start handling this complexity in a biologically meaningful way. The studies presented in this thesis are among the first applications of these tools to EEG data. 


\subsubsection{The principles of permutation tests}

The underlying machinery behind the variety of statistical tools that will be discussed below is permutation testing. The basic idea of this method is about a hundred years old, but only recently thanks to the increase in computing power it became possible to implement it.

The statistical problem that most EEG studies come down to is determining whether there is a difference between two sets of EEG data. These sets may belong to the same single subject (for example pre-stimulus baseline and response to a stimulus), to two groups of subjects (for example patients and healthy controls) or to the same group of subjects under different conditions (for instance before and after treatment with TMS). There are many aspects in which the two datasets may differ. Trivially, they will always differ for finite number of trials because of the noise that is different in different realizations of the measurement. So, what is usually tested is whether the two datasets come from the same distribution with respect to some parameters of interest. The hypothesis that this is indeed the case (the null hypothesis) is usually more convenient to test than the alternative hypothesis (the datasets come from different distributions) because its predictions are easier to verify. Moreover, we may apply manipulations to the two datasets under the assumption that they are the same and if the outcome is not consistent with this assumption, we may reject the null hypothesis even without going into the details of what exactly caused the inconsistency. In neuroimaging research, however, the details of the manipulations are usually of importance because they may lead to a physiological interpretation of the differences in the data.

In particular, under the null hypothesis some of the labeling of the data may be considered arbitrary and if the null hypothesis holds these arbitrary labels may be randomly exchanged or permuted without changing the statistical properties of the whole dataset. For instance, when we compare a group of patients with a group of healthy controls with respect to the amplitude of the N20 peak of the somatosensory evoked potential, the null hypothesis would be that there is no difference between patients and controls and hence that the data in both groups come from the same distribution. Therefore, if we randomly reshuffle subjects between the two groups while preserving the group size, or equivalently exchange labels between randomly 
chosen pairs of patient and healthy control, the disparity between mean N20 amplitudes of the original two groups is not expected to be different from the disparity between the mean amplitudes of the shuffled groups. If we perform just one shuffling it would be hard to say to which extend the single 'shuffled disparity' is different from the original disparity. However, given that the datasets consist of sufficient number of items whose labels can be permuted, a multitude of possible permutations exists and by taking a representative sample of this multitude an estimate for distribution of the 'shuffled disparity' can be attained. One can then compute the probability that the original disparity belongs to the same distribution. This probability - the p-value - represents the probability that the null hypothesis is true, a small p-value indicates that the null-hypothesis is not likely. According to widely accepted convention a p-value below 0.05 , or below 0.01 when more conservative approach is taken, is a justification for rejecting the null hypothesis. It should be stressed that failure to reject the null hypothesis is not equivalent to accepting that the datasets are the same. When the null hypothesis is not rejected, we merely cannot make a statistically justified decision due to insufficient evidence.

The same problem of determining whether two sets of numbers such as N20 amplitudes of patients and healthy controls come from a different distribution can be handled also with parametric statistical tools such as Student's t-test. However, these tools rely on more stringent assumptions about the distribution of the parameter under study. While these assumptions are quite likely to be true in simple cases such as the one presented above, they can be violated in more complicated cases or more complex data types such as power and coherence. Testing the distributional assumptions in each case and tailoring the appropriate tools to handle the different kinds of possible violations is a tedious and complicated process. The non-parametric permutation based approach makes it possible in a wide variety of cases to perform statistical tests in a way which is simpler and easily accessible by researchers with only basic understanding of statistics. Moreover, releasing the necessity of a strict distributional requirement in the data allows more freedom in choosing the statistic to be examined. The statistic that would be the most interesting for a biologist is the one that optimally captures the property in which the datasets being compared are expected to differ. However, with the conventional parametric methods such statistics could not always be used because their distribution was not suitable for these methods. 


\subsubsection{Multiple comparisons problem}

The simple example presented above was examining the amplitude of a single waveform at a single pre-determined time point. However, our purpose presented earlier is to develop tools capable of finding effects in the data with minimal prior information and minimal pre-selection and averaging. In order to do this, one should perform statistical tests on multiple variables in our multidimensional dataset (for instance, compare all the electrodes and all the timeframes in scalp ERP). A problem immediately arising when doing this is that of multiple comparisons. As discussed in the previous paragraph, when we reject the null hypothesis, we allow some small probability of error determined by convention. If this probability is 0.05 , then in $5 \%$ of all tests that we perform, the null hypothesis will be rejected, while in reality it holds. This problem is tolerable when a single test per study is performed. However, the number of tests required to compare two ERP voltage patterns recorded with 64 electrodes during $30 \mathrm{~ms}$ with sampling rate of $2000 \mathrm{~Hz}$ is

Therefore, when we perform such a comparison and the null hypothesis holds we expect to reject the null hypothesis for $\square$ cases. In other words, we would conclude that there are quite a lot of differences while in reality there are none. Moreover, the chance that the null hypothesis will not be rejected in any of the tests is $(1-0.05)^{\wedge} 3840=\square$. Thus, for all practical purposes we a priori know that the null hypothesis will be rejected, which makes the statistical test meaningless. The calculations above are not completely valid in our case, since they assume that all samples to be tested are independent whereas in reality neighboring time points in an ERP waveform are correlated, but they do illustrate the multiple comparisons problem (MCP).

A common solution to MCP is the Bonferroni correction. The idea is to lower the probability of falsely rejecting the null hypothesis in each of the individual tests so that the probability of falsely rejecting it in any of the tests in a group will be the conventional 0.05 or 0.01 . This is done simply by dividing the original p-value threshold by the number of tests performed. So in our example the threshold should be $0.05 / 3840=0.000013$. The problem is that the significance of actual physiological effects rarely reaches such low p-values, especially with relatively small numbers of subjects. Thus, the ability of Bonferroni corrected group of tests to discover physiological effects (also called statistical power) is very low. There are additional 
less stringent correction techniques based on similar principles, but they all fail when the number of tests performed is as large as in most neuroimaging applications.

\subsubsection{Clustering based approach}

The fundamental limitation of all attempts to correct the threshold of individual 'point-wise' tests in the context of neuroimaging is the assumption that physiologically significant effects differ from random noise in some feature that can be seen at the level of individual data points. Theoretically there are many parameters characterizing the distribution of a value at a particular data point across repetitions (subjects or trials). However, in practice we can expect mostly differences in either the amplitude of the effect or its consistency across repetitions. These are the two features summarized by conventional T-statistics. The problem is that in most cases the differences in these features between physiological effects and noise are not dramatic enough to pass significance thresholds corrected for multiple comparisons.

To find another way, let us think about what we, as researchers, do while looking at neuroimaging data and trying to find physiological effects in the noise. Figure 7 shows several examples encountered in the context of neuroimaging. The common motive of these examples is that the physiological effects have characteristic scales in time, space and frequency which are very likely to differ from the corresponding scales of noise. Physiological scalp patterns in human EEG are created by sources in the brain that always affect more than one EEG electrode (given that there are enough electrodes to adequately sample the physiological scalp patterns). Conversely measurement noise affects one electrode at a time. There are also artifacts affecting several electrodes, such as eye blinks, but these can be dealt with by other tools described in previous subsections. Furthermore, physiological effects, unlike measurement noise, usually last for several consecutive timeframes. In frequency, physiological signals and effects associated with them span relatively wide frequency range of at least several $\mathrm{Hz}$, unlike line noise, for instance, which is localized to a very narrow band. These scale differences cannot be measured by looking at each data point separately. However, they can be easily evaluated by the eye of a human observer. A computer routine can mimic this human approach by looking for clusters in the data. Clusters in the present context are assemblies of points adjacent in at least one of the dimensions of interest that jointly exhibit the same effect. The 
nonparametric cluster-based statistical test is, therefore, performed in two steps. At the first step the effect of interested is tested for all the points in the dataset. The test can be either non-parametric permutation test or parametric test. This produces for each point a statistic that tells us to what extent we can be confident that the effect of interest is expressed at this point. Let us call it point-specific statistic. We then threshold the point-specific statistic to find the points expressing the effect of interest at a sufficient level. The exact test performed at the point level and the value of the threshold do not affect the validity of the next level of the test as will become clear later. However, modifying it can adjust the sensitivity of the test to different kind of effects. By default the threshold corresponding to $p$-value of 0.05 can be taken. At the second step the points that crossed the threshold are grouped into clusters in such a way that, within every cluster, they form a set that is connected along all the dimensions of interest. The definition of connectedness along time or frequency is clear and intuitive. Connectedness in space is clear for volumetric data such as current source density reconstructions. For EEG scalp data, however, neighborhood relations between electrodes have to be defined in order to derive from them the definition of connectedness in space. After clusters are identified, each cluster is assigned a clusterlevel statistic, whose value equals the sum of the point-specific statistics for all the point in the cluster. Thus, the cluster-level statistic depends on both the extent of the cluster and the magnitudes of the point-specific t-statistics at the points that belong to this cluster (Takashima et al., 2006). Then a permutation test is performed by repeating the whole clustering process for a large number of datasets with permuted labels. For each of these permuted datasets the maximal value of the cluster-level statistic is recorded and the values of the cluster-level statistics that were obtained for the original labeling are compared against the distribution of these maximal values. This comparison results in p-value being assigned to each cluster. Finally, only clusters with p-values below cluster-level significance threshold (again 0.05 or 0.01 for conservative approach) are accepted as significant. If no such clusters are found, the null hypothesis cannot be rejected. This procedure solves the MCP because there is only a single test statistic - the cluster-level statistic instead of one test statistic for every data point. 

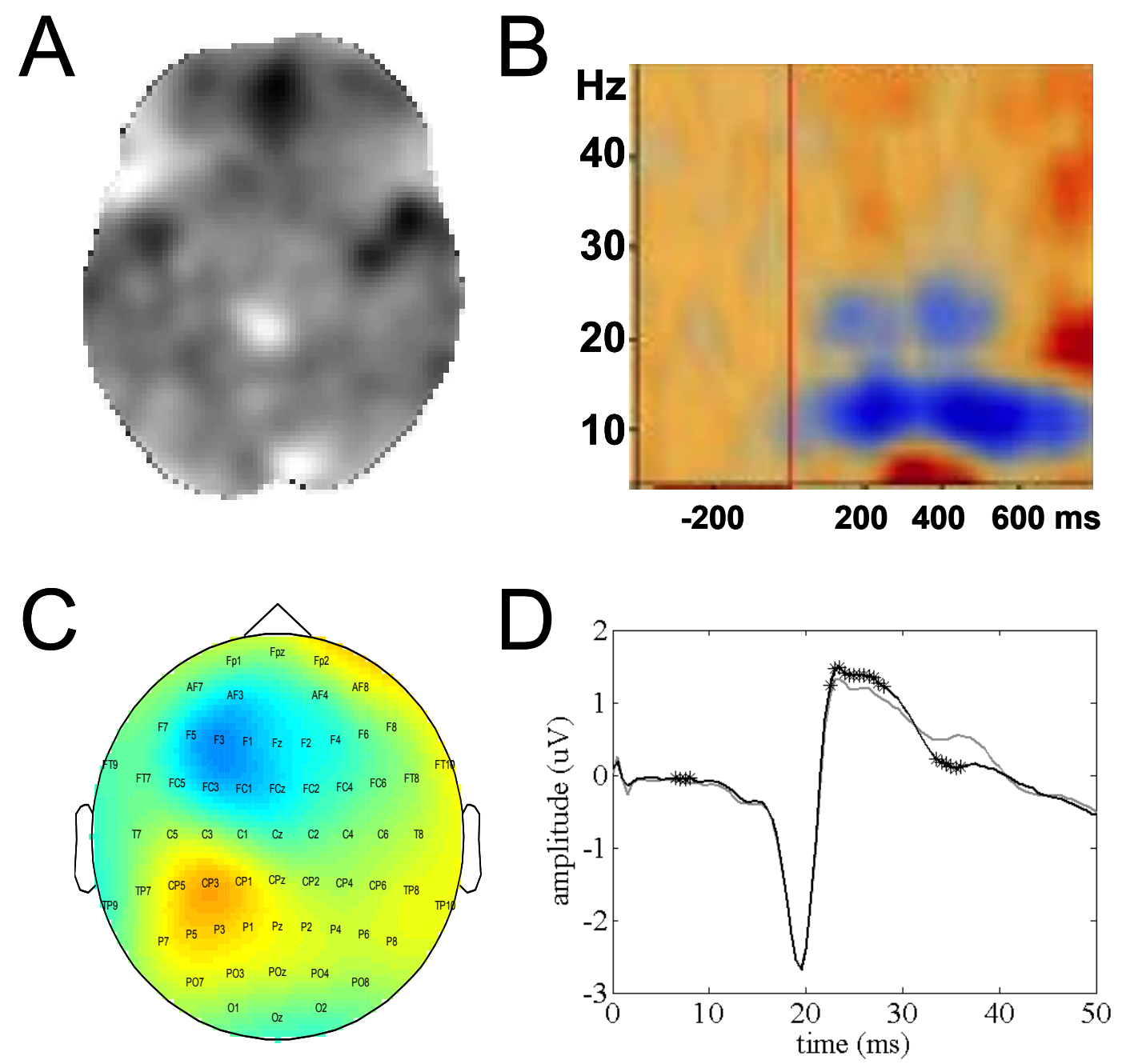

Figure 7. Examples of neuroimaging data where human eye can easily detect physiological effects based on spatial and/or temporal continuity. A. fMRI data slice. B. Time-frequency decomposition of EEG data. C. Scalp potential map of EEG data. D. ERP waveforms (asterisks indicate significant point-by-point difference).

To summarize, the algorithm for nonparametric cluster based statistical test is as follows:

1. Compute a point-specific statistic of interest for every point in the dataset.

2. Threshold the point-specific statistic at some value.

3. Compute connected clusters of suprathreshold points.

4. Compute cluster-level statistic for each cluster by summation of the pointspecific statistic values of all the points belonging to the cluster. 
5. Create a large number of datasets with permuted labels. For each of these datasets repeat the steps 1-4 and construct a histogram of the maximal clusterlevel statistic value obtained.

6. From the cluster-level statistic that was actually observed and the histogram in step 5, calculate the proportion of label permutations that resulted in a larger cluster-level statistic than the one that was actually observed. This proportion is the p-value.

7. If the p-value is smaller than the critical alpha-level, then reject the null hypothesis.

\subsubsection{Adapting and extending clustering based tests for specific applications}

As discussed above, the nonparametric permutation-based framework makes it possible to test for a difference between datasets with respect to any statistic. The statistic being tested in the above algorithm is the cluster-level statistic for the whole dataset. Steps 1-4 of the algorithm are just the procedure for computing this statistic and not a statistical test. Although the point-specific statistic of step 1 is usually some common statistic (such as Student's T), it does not have to be the case. The thresholding of the point-specific statistic is also just a step for computing the clusterlevel statistic. Thus, the threshold value does not affect the false alarm rate of the whole test. However, it does have an effect. If the threshold value at step 2 is low, there will be many suprathreshold points and large clusters consisting of points with weak effect. Conversely, if the threshold value is high, there will be small clusters consisting of points with strong effect. Thus low thresholds makes the test more sensitive to weak but extensive effects ('large spots') and high threshold - to strong but limited in their extent ('spikes'). Changing the point-specific threshold is not the only way to tweak the test to adjust it to the effect of interest. Although, we declared eliminating data selection and averaging as our goal, in practice we analyze only some window of interest in time and frequency and in many cases a region of interest in space. The larger this window/region if interest is, the larger will be the values of cluster level statistic that will be obtained by chance in the permuted datasets. Thus, the test will become less sensitive to physiological effects that are short in time, narrow in frequency or small in space. The validity of the test will not be affected but its sensitivity (statistical power) will decrease. Thus, having an a priori expectation about the range in parameter space where an effect is expected can improve our 
ability to discover it. This also opens the door for abuse of the method, since one can run the test, find a non-significant cluster somewhere and then tweak the parameters of the test to 'discover' this cluster (for instance, put the time and frequency limits tightly around the cluster). This is statistically wrong and equivalent to performing 100 t-tests with p-value 0.05 and publishing a paper about the five tests that were significant by chance. To summarize, steps $1-4$ of the test can be tweaked in any way to increase the test's power for discovering the effect of interest as long as this is done based on a priori expectations before actually running the test.

Until now we discussed comparison of two datasets. However, the nonparametric framework can be extended to handle more complicated cases as long as there is a well defined procedure for computing point-specific statistic and exchanging the condition labels under the null hypothesis. An example that is of interest for the present thesis is testing for significant regression with an external parameter. For example, in one of the studies presented below, we were interested to test the hypothesis that the EEG response to TMS is correlated with TMS amplitude and find clusters of data points expressing this significant correlation. For each subject four TMS intensities were tested. Our point-specific statistic was the t-statistic for dependent samples correlation computed across the subjects. The null hypothesis states that there is no correlation between the TMS intensity and the amplitude of the response. Thus the labels could be permuted within subjects between the different intensities. With these definitions we could perform the steps 1-7 of the algorithm and find the effects we were looking for.

In summary, the non-parametric clustering-based permutation test framework provides us with a very flexible, intuitive and statistically sound tool suitable for testing for variety of effects with minimal prior knowledge and distributional assumptions. These properties made this framework very suitable for our project because most of the effects we studied are novel and there are no or few prior expectations about them that can be derived from the literature. It is our impression that the clustering based tests in many cases discover effects that appear physiological based on features that the test itself is blind to. For instance, in many cases the test discovered a dipolar pattern consisting of two simultaneous significant clusters positive and negative, whose locations were consistent with one underlying single tangential dipolar source. Since positive and negative effects are tested independently, 
this is a strong confirmation for the physiological nature of the effect. Also in spectral analysis effects were found at physiological frequencies and had sensible scalp patterns though none of this was programmed into the test in advance. The nonparametric tests are just beginning their way into EEG research, although they are well known in fMRI analysis (Nichols and Holmes, 2002). We hope that our studies will be of interest not only as examples of using the tests to study the effects of TMS but also as examples of using well-defined effects of TMS to confirm the validity of nonparametric tests. 


\section{The objectives of the present research}

The starting point for all the lines of research described in the present thesis was developing a closed-loop system for modification of brain connectivity and function based on TMS. In all TMS studies appearing in the literature the stimulation protocol was determined in advance and did not depend on the magnitude of changes that the stimulation induced in the brain. We believe that with better understanding the way TMS-based stimulation protocols impact the neural circuitry, the effect of TMS can be monitored in real time and used in a closed loop fashion in order to maximize the effect of the stimulation and increase its specificity. A schematic drawing of a hypothetical system based on this principle is shown in Figure 8. The system consists of a TMS device, EEG/EMG measuring electrodes, amplifier box and a computer which analyzes the recorded EEG and EMG signals and controls the parameters of magnetic stimulation. In order to realize this idea a system should include two major components. First, TMS-based protocols should be developed that can specifically target the neural circuits of interest. Secondly, methodology for monitoring the effect of these protocols has to be developed that can provide us with the information relevant for fine-tuning the stimulation parameters. The three studies appearing in the present thesis are all aimed at developing possible components of such a system. Building a complete system is a project too ambitious to be realized within the framework of a Ph.D. Nevertheless, in our opinion it comprises an interesting research program which can lead to discoveries important in their own right event if the project as a whole is never fully realized. 




Figure 8. Scheme of a hypothetical closed-loop TMS system. EEG and EMG signals are analyzed in real time and used to control the stimulation system. 


\section{Description of the studies in the present thesis}

One of our primary objectives from the beginning of the project has been developing methods for the analysis of EEG responses evoked by TMS. Until now the most common method for assessing the effect of TMS on cortical connectivity and excitability was examining the amplitude of MEP. This method is inherently limited to the primary motor cortex (M1). It is quite possible, however, that the physiological properties of M1 are different from other cortical areas. In addition, when TMS is applied for treatment purposes to areas other than M1, there is no way to monitor its effect since it does not evoke MEPs. Therefore, a more general method is necessary. Examining TMS evoked ERPs can be such a method, since EEG electrodes can be placed practically anywhere on the scalp. A well developed TMS-EEG integration technology can potentially have much wider applications than just studying TMS effects. The present ERP technology only allows studying cortical areas that are consistently involved in processing sensory stimuli or in preparation of motor responses. Therefore, it is customary to develop a particular experimental paradigm and ask the question what cortical areas are activated by this paradigm. The opposite process of selecting a particular cortical area and finding out its physiological properties, connectivity and function is rarely done. By combining EEG and TMS it might be possible to systematically study these properties in all cortical areas accessible to TMS stimulation and build a map of functional connectivity of the human cortex. Such a map cannot answer all our questions about brain function but it can play a significant role in neuroscience research similar to the role that human genome sequence presently plays in genetic research (Sporns et al., 2005). Particularly it can serve for diagnostic purposes after pathologies in functional connectivity associated with different neurological and psychiatric disorders are characterized (Massimini et al., 2005b).

Our initial plan was to perform our own recordings of EEG responses to TMS and analyze them. However, preliminary experiments showed already at very early stages that our EEG system is unsuitable for combination with TMS. This was a 12-bit system prone to long saturations and large artifacts following a TMS pulse. We also tested several newer systems but in all of them we found large artifacts that we could not subtract at the time. Therefore, we contacted the research group in Finland and 
established collaboration for analysis of the data recorded in their previously published experiment (Komssi et al., 2004). After examining the data we found that although it was recorded with the hardware cutoff circuit, the early stages of the responses were in many subjects contaminated by artifacts, albeit of lower amplitude than in systems we tested before. In collaboration with German company Megis Software $\mathrm{GmbH}$, we adapted a previously developed method for correcting eye movement artifacts in EEG (Berg and Scherg, 1994a) to correcting EEG artifacts. Using this method combined with the nonparametric statistical approach described above we were able to recover and analyze the earliest responses to TMS stimulation at left and right M1. My contribution to this study was in initiating the additional analysis of the data of Komssi et al. (2004), developing the Matlab scripts for integration of BESA and Fieldtrip for this analysis and performing the actual analysis and interpretation of the results in collaboration with Dr Michael Scherg. The results of this study are presented in the first part of the Results section.

The next problem we wished to address was characterization of long term effect of TMS based protocols using EEG. The idea to develop a protocol based on Hebbian pairing of a sensory stimulus with TMS came up very early in our discussions. Only later we discovered that some experiments in this direction have already been published by the German group headed by Dr. Joseph Classen (Stefan et al., 2000; Stefan et al., 2002). This research has already been described above. We decided to contact Dr. Classen and learn from his experiences in this field in order to establish our own research program. From these personal contacts we learned about a new project in his lab that was not yet published at the time. This project was aimed at analysis of effect of paired associative stimulation on somatosensory evoked potentials (Wolters et al., 2005). As mentioned above, a variant of PAS protocol originally developed for M1 was used. In this protocol electrical stimulation of median or ulnar nerve was paired with magnetic stimulation of S1. Wolters at al. used clinical SSEP recording with a single channel (electrode above S1 referenced to the forehead) and examined the amplitudes of early peaks N20 and P25. They tested a range of intervals between the peripheral stimulation and TMS. The timing of the TMS pulse was defined relative to subject's N20 peak, which marks the time of arrival of the somatosensory stimulus to S1 (Nuwer et al., 1994b). The conclusions of the study were that while N20 amplitude is not affected by PAS, there is a significant effect on the P25 amplitude. This effect depended on the PAS-interval in a manner 
consistent with the properties of STDP and previous results of M1 PAS (Figure 1). When the TMS pulse was delivered close to the N20 peak (the optimal timing found by interpolation was $2.5 \mathrm{~ms}$ before N20) P25 amplitude was increased following PAS for at least an hour. When TMS was applied 20ms before N20 i.e. around the time of peripheral stimulus, a decrease in P25 amplitude was induced. The effect was specific both to TMS stimulus location (stimulation above M1 did not affect P25 amplitude) and to peripheral stimulus location (PAS affected MN-SSEP but not SSEPs evoked by stimulating the tibial nerve innervating the leg). It became immediately clear to us that the effect of S1 PAS was is a very suitable subject for study with multichannel EEG, since by definition this effect has a clear EEG correlate unlike other known long-term effects of TMS. On the other hand single channel clinical SSEP recording does not provide enough information about the scalp pattern of the effect to determine its regional and laminar origin. These considerations led us naturally to collaboration with the group of Dr. Classen. The result of this collaboration is presented in the second part of the Results section. Using 64-channel EEG system we were able to pinpoint the exact cortical area affected by S1 PAS and based on results from the literature conclude with high degree of confidence which cortical layer is affected. Surprisingly for two of the intervals we tested (one of which was originally planned as a control condition) we found two different significant effects that localized to different cortical areas. In addition, we studied the effect of PAS on the tactile discrimination ability of the subjects. Also in this behavioral study significant affects were found and one of them was correlated with changes in MN-SSEP activity. The particular novelty of our study was that the effects were discovered with the data driven nonparametric statistical approach. We were therefore able to not only confirm part of the original findings of Wolters et al. but also reveal new effects not expected in advance. My contribution to this study was in initiating the use of multichannel EEG for investigating PAS effects, performing the experiments in collaboration with Daniel Zeller, developing the analysis methods, writing the Matlab scripts for integration between BESA and Fieldtrip, and performing the analysis and interpretation of the results under the supervision of Dr. Classen.

The final study, appearing in the third part of the Results section, dealt with the phenomenon of variability in MEP amplitudes induced by TMS under the same conditions. Highly variable responses of a neural system to identical stimuli have been previously observed in many cased and it has been suggested that this variability 
can be used for developing a new kind of learning protocols (Shahaf and Marom, 2001). We were, therefore, interested in studying the origins of this variability addressing the question whether it is influenced by the TMS stimuli or only by spontaneous changes in the excitability of the motor pathway. Preliminary experiments supported the hypothesis that the variability is intrinsic rather than TMS driven. Based on this assumption we performed EEG recordings while delivering a long series of TMS pulses and examined the EEG segments recorded before the TMS in order to find correlates of the subsequent MEP response amplitude. Also in this study the data driven approach was instrumental, since we had no prior expectation for the kind of correlate we expect to find. A correlate was indeed found and its pattern both in space and in frequency makes it highly likely that it reflect physiological processes in the sensorymotor cortex. Our finding also support previous studies about the importance of the brain's ongoing activity in shaping the response to a stimulus and provides a novel way to study ongoing activity in intact human brain. My contribution to this study was in suggesting the idea and developing the experimental protocol, performing the experiments in collaboration with Daniel Zeller, developing the analysis methods, writing the Matlab scripts for integration between BESA and Fieldtrip, and performing the analysis and interpretation of the results under the supervision of Dr. Classen. 


\section{Methods}

In the present section we will discuss the general aspects of the methodology used in the studies presented below. Since the three studies were different in their aims, the specific methodological details for each of them are presented in the corresponding chapter.

\subsection{Experimental setup and data acquisition}

The data analyzed in the present thesis has been acquired in two laboratories. The EEG responses to TMS at the left and right M1 were recorded at Biomag Lab at the University of Helsinki using the special sample-and-hold circuit for blocking TMS artifacts (Virtanen et al., 1999). The system has been described in several research papers (Ilmoniemi et al., 1997) (Komssi et al., 2002) (Komssi et al., 2004). Since the author participated only in data analysis and not in the experiments, the experimental setup of the Biomag Lab will not be described in details here.

The study of the effect of the paired associative stimulation and the study of EEG correlates of MEP variability have been performed in the Human Cortical Physiology Laboratory, Department of Neurology University of Wuerzburg, Germany. This laboratory was equipped with state of the art hardware and software for performing TMS experiments and neurophysiological data acquisition. The author participated in setting up a new EEG recording system in the lab and integrating it into the experimental setup. The final setup used for both studies consisted of the following components.

Magstim 200 magnetic stimulator (Magstim, Whitland, Dyfed, UK) - TMS device used for cortical stimulation. The device is capable of delivering single pulses with recharge time of more than a second. Thus, it is not suitable for rTMS but quite adequate for the studies of the present thesis where only low stimulation rates were used. The device was equipped with custom-made flat figure-eight shaped magnetic coil (diameter of each wing: $70 \mathrm{~mm}$ ). Custom made coils unlike standard coils are not covered with protective plastic encasement but only wrapped with thin insulating band. This reduces the distance between the conducting metal and the scalp and allows stimulating more focally and reducing the stimulation intensity. 
Electrical nerve stimulator (Digitimer D7AH, Digitimer, Welwyn Garden, Herthfordshire, UK) - the device used for electrical stimulation of the median nerve for PAS and MN-SSEP recording.

64-channel Quickamp EEG system (Brain Products GmbH, Munich, Germany). This is a portable EEG system that was deployed in the lab before each experiment. There was no special recording chamber. The Quickamp system incorporates proprietary noise reduction technology called "actiShield". Each electrode cable is surrounded by a shielding which connects to the zero in capacitance of the amplifier. Channel by channel environmental noise picked up by the shielding is fed back to the amplifier and used to actively suppress any influence of the noise on the data of interest. In order to take advantage of this technology each electrode had to be connected separately to the amplifier. The electrodes were fixed on the head with elastic cap (EASYCAP GmbH, Herrsching-Breitbrunn, Germany). Unlike in some other EEG systems the electrodes were not integrated with the cap but attached on the outside with special plastic holders. We had two caps with different sizes and in case of the need to switch the cap size or wash the electrodes the system was taken apart and reassembled. In order to assist the cap assembly and connecting the electrodes, they were divided into eight bundles of eight. The electrodes were labeled on both ends to ensure correct connection. The EEG system was connected to a dedicated computer running "BrainVision Recorder" software (Brain Products GmbH, Munich, Germany). In MEP variability study we also used the Quickamp system for EMG recording so that both EEG and EMG data will be synchronized at the same system.

Recording and control system consisting of a second computer running "Signal" software (Cambridge Electronics Design, Cambridge, UK), analog amplifier (CED1902, Cambridge Electronics Design, Cambridge, UK), A/D converter (CED1401 plus, Cambridge Electronics Design, Cambridge, UK) and pulse generator (Master-8, A.M.P.I., Jerusalem, Israel). The Signal software makes it possible to define stimulation paradigms and record physiological signals (other than the EEG). Master- 8 is a programmable pulse generator extending the capability of Signal to precisely time the activation of different components of the system. 
3D navigation system (Brainsight, Rogue research, Montreal, Canada). The system (Figure 9) consists of two infrared cameras combined with infrared emitters, trackers marked with special reflective spheres that can be seen by the camera and dedicated computer running Mac OS and Brainsight software. One tracker is fixed to the head of the subject using plastic glasses and a second tracker is fixed to the TMS coil. At the beginning of the experiment the coil is calibrated using a special calibrator device in order to indicate for the navigation system where the center of the coil is in relation to the tracker. The subject is registered to an anatomical MRI image by pointing at several reference points on the head such as bridge of the nose, tip of the chin, preauricular points, top of the head and the inion with a pointer tracked by the Brainsight system. After the registration the system starts tracking the position of the pointer in relation to the head and showing it in relation to the MRI scan. The quality of the registration can then be verified by pointing at several points on the head and checking whether they are displayed correctly. The coil is also tracked by Brainsight in real time. Brainsight performs a $3 \mathrm{D}$ reconstruction of the cortical surface and presents the position of the coil relative to this surface. This was used during the experiment while searching for the optimal stimulation point to systematically scan the motor area. After the stimulation point is determined it can be stored in the software and Brainsight provides graphical interface for repositioning the coil at the same point again. This feature was used for determining the stimulation spot and then returning to it after putting the EEG cap on when the markings in the skin could not be seen any more. Also during the PAS intervention and delivering a series of stimuli in the MEP variability study the experimenter constantly watched the positioning screen and made sure that the coil position remained constant. Although it required some effort and concentration from the experimenter, this was the solution of choice for us to keep the position of the coil relative to the subject's head constant. Other solutions usually involve fixing the subject's head to a frame. This practice is not comfortable for the subject, does not prevent movements completely and is not suitable for long periods of time such as needed for our experiments. The Brainsight system was also used for recording electrode positions for each experiment. This was done using the tracked pointer. 

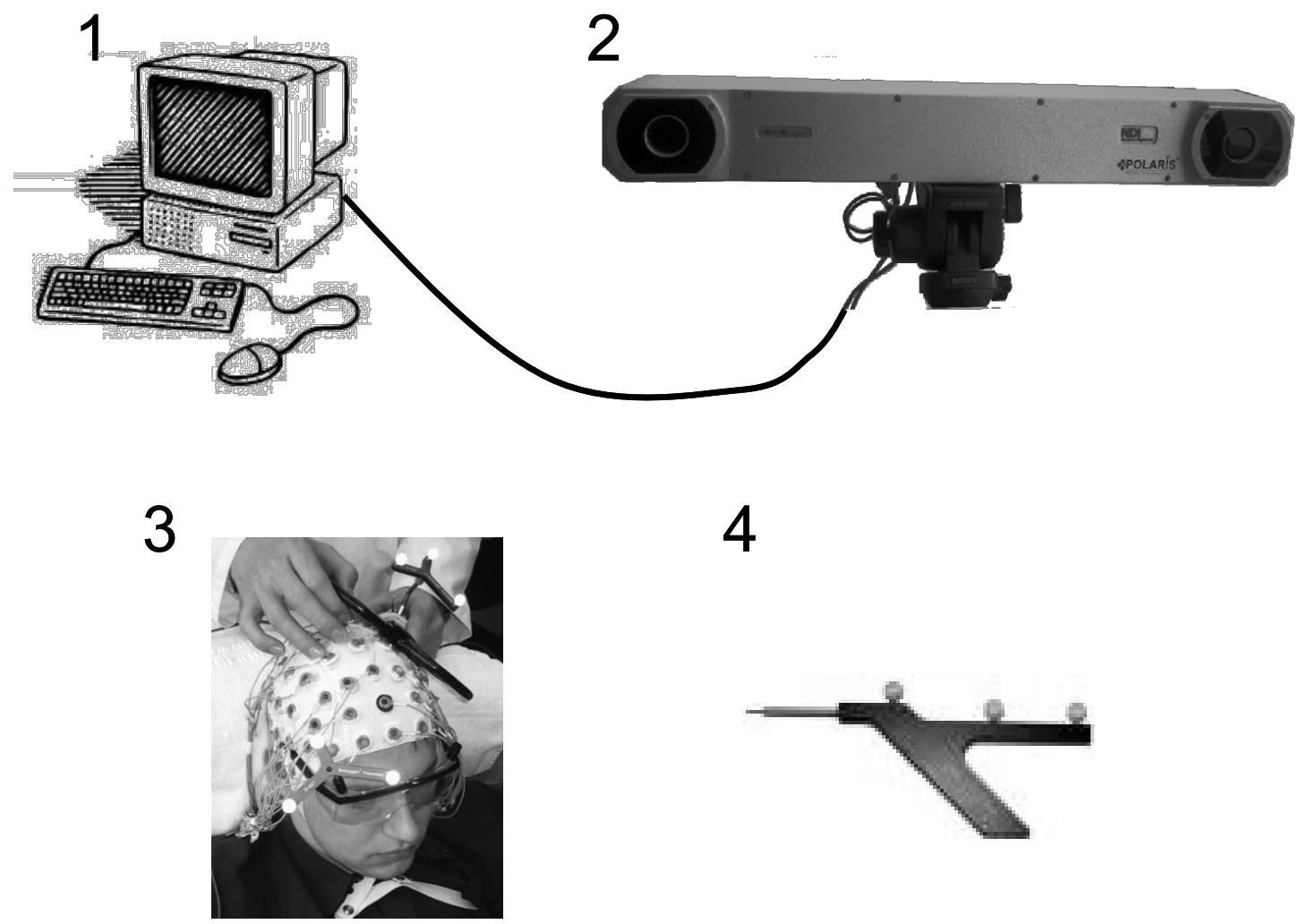

Figure 9. Brainsight navigation system (1) Mac OS computer running the Brainsight software (2) Infrared camera (3) Trackers on subject's head and on the TMS coil (4) Trackable pointer used for registration and electrode position measurement

Although, it is preferable that the subjects are registered in Brainsight to their own MRI scans, this is not critical for the experiments in our studies. We found stimulation sites based on physiological rather than anatomical criteria and the ability to maintain constant coil position using Brainsight does not depend on the MRI scan used. Nevertheless, in the PAS study all the subjects were scanned and their own scans were used for the experiments. In the MEP variability study some of the subjects were not scanned. In these cases we matched to the subject one of the available scans based on the head circumferences in coronal and transverse planes.

Figure 10 shows the diagrams of the experimental system used for the PAS study (A) and MEP variability study (B). The Brainsight system was used in both studies in a similar way. Figure 11 shows the general view of the setup during preparation for an experiment. 

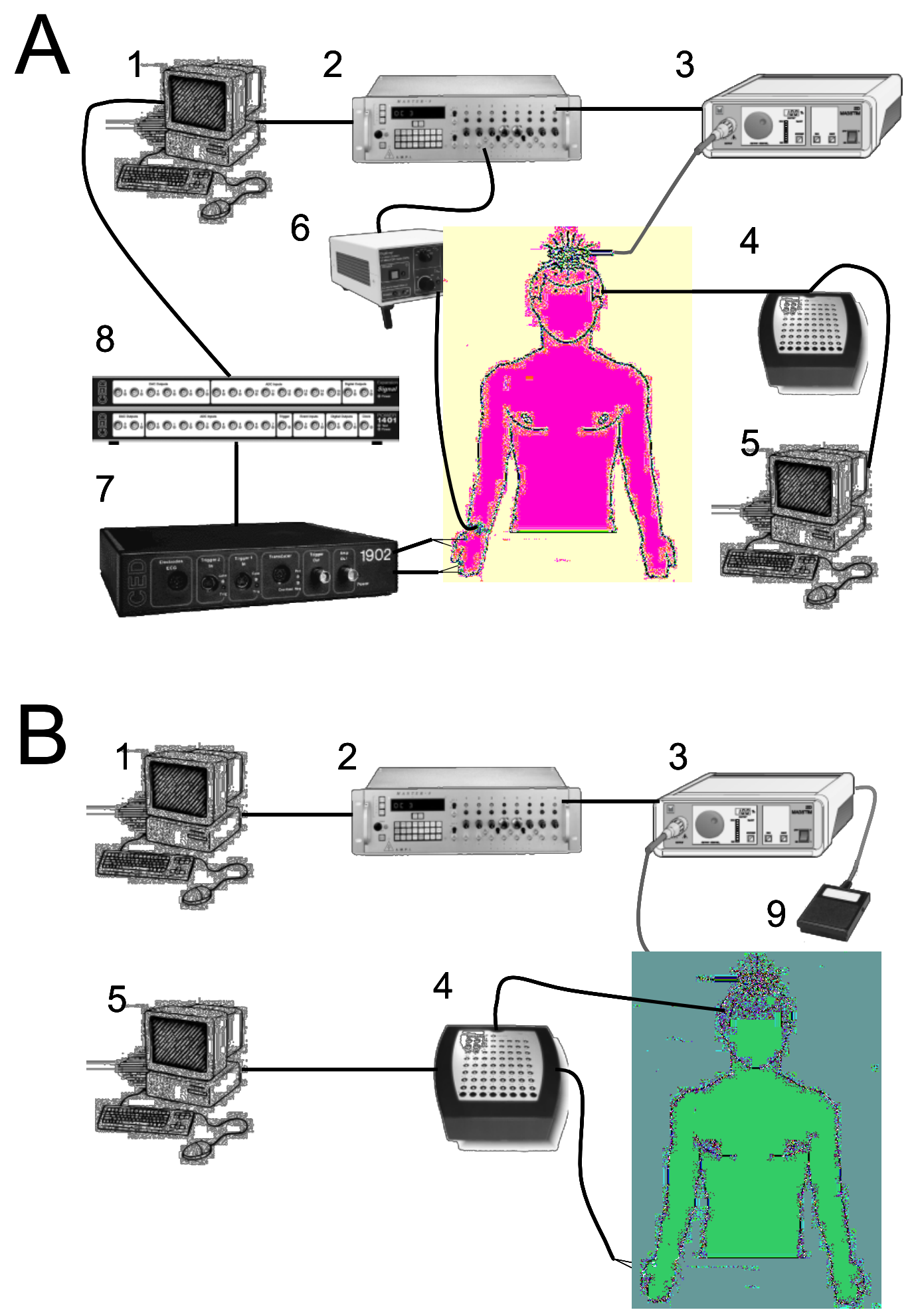

Figure 10. Experimental setup used for the PAS study (A) and MEP variability study (B). (1) Recording and control computer running the "Signal" software (2) Master-8 pulse generator (3) Magstim 200 magnetic stimulator (4) Quickamp EEG system (5) EEG recording computer running "BrainVision Recorder" software (6) Electrical nerve stimulator Digitimer D7AH (7) CED1902 analog amplifier (8) CED1401 plus A/D converter (9) Foot pedal 


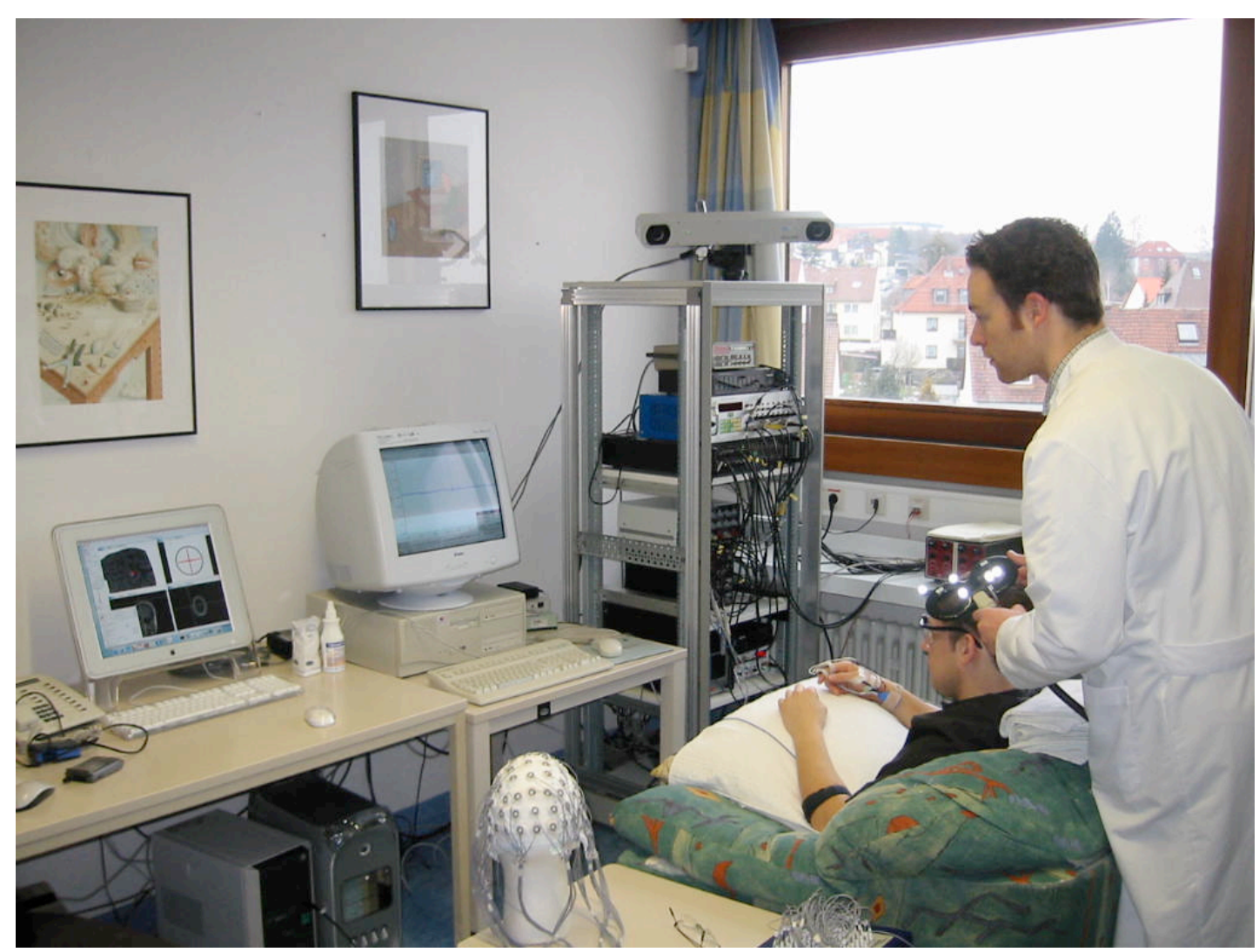

Figure 11. General view of the experimental setup during an experiment combining magnetic stimulation and EEG recording. On the picture from left to right: Brainsight navigation computer, Signal computer controlling recording and stimulation, equipment tower with the navigation camera on top of it. In the middle at the bottom of the picture - EEG cap and Quickamp recording system.

\subsection{Experimental procedures}

During the experiment the subjects were seated in a comfortable chair. The session started with fixing the EMG electrodes to the subject's right hand. The recording electrode was fixed at the belly of abductor policis brevis (APB) muscle and the reference electrode at the base of the metacarpo-phalangeal joint of the thumb. In the PAS study the electrodes for recording sensory nerve action potentials (SNAPs) were fixed to the index finger and the stimulating electrode was fixed at the wrist.

In the PAS study the next step was measuring the threshold for median nerve stimulation. We started with very low intensity and raised it slowly while triggering stimulation pulses manually and asking the subject whether the stimulation was felt in the fingers or in the palm of the hand (which would indicate activation of the median nerve). When the subject reported feeling the stimulation the intensity was lowered to 
find the threshold. The threshold for motor activation, which was always higher than the sensory threshold, was found in a similar way.

Then we put the tracker glasses on the subject's head and performed registration to the Brainsight system. After verifying the registration we searched for the optimal point for stimulating the APB representation in M1 (the motor hotspot). The approximate search area was determined based on the MRI scan. The central sulcus could be seen clearly in all subjects. The hand representation was usually located near the Omega shaped knob in precentral gyrus (Yousry et al., 1997). We triggered the TMS pulses with a foot pedal with intervals of at least 5 seconds and moved the coil between pulses along the precentral gyrus to find the location where largest MEPs could be elicited with the lowest stimulation amplitude. Once such location was found, we also probed the anterior-posterior direction to find the optimal position. Finally we performed small movements around the candidate location to make sure that no better position can be found around it. After making sure that the candidate position is indeed optimal we saved it in Brainsight. During this part of the experiment the subject was instructed to relax the right hand. The relaxation was monitoring by checking the baseline part of the EMG recording and in cases when subject did not relax properly we used a loudspeaker to let the subject hear his own EMG and relax better using this feedback.

For the PAS study we also determined the optimal position for stimulating the somatosensory hand area (somatosensory hotspot). This position was defined as a point $2 \mathrm{~cm}$ posterior to the motor hotspot. To find this position we first placed the coil at the motor hotspot using Brainsight and marked position of the edge of the coil on the scalp using soft-tipped pen. We then replaced the coil with its cardboard replica having a hole in the center. The edge or the replica was positioned according to the previously made markings and the hole was used to mark the position of the center of the coil which is the actual motor hotspot. We then measured with a measuring tape $2 \mathrm{~cm}$ posterior to the marked spot and marked it with a different color. The coil replica was repositioned with the hole above the somatosensory hotspot and oriented in parallel to the coil position at the motor hotspot. The edge position was marked on the scalp. These markings were used to position the real coil in the same way and the new position was recorded in Brainsight. Figure 12 shows the stages of this process. 


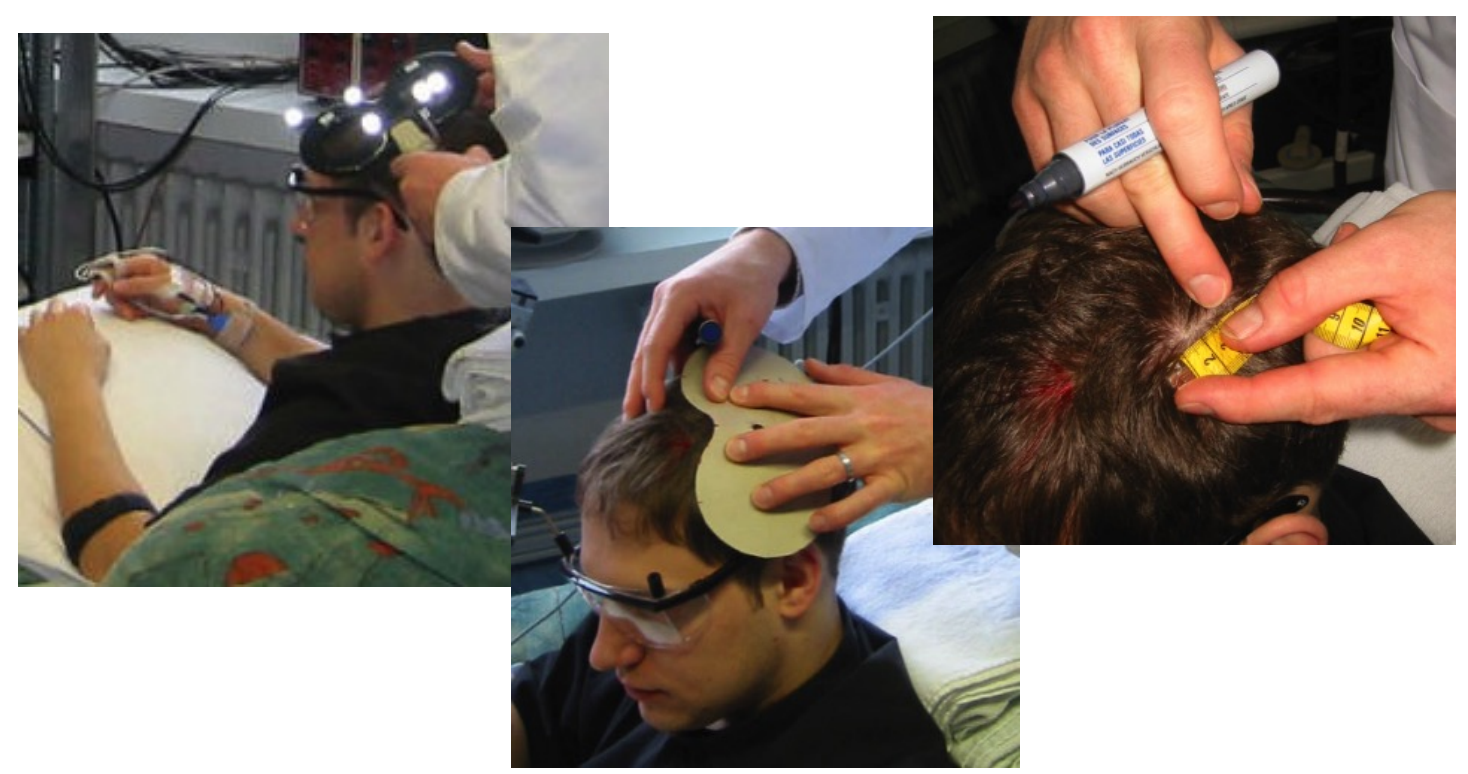

Figure 12. Determining the position of motor and somatosensory 'hotspot' for a PAS experiment (see text for details). From left to right: (1) localization of M1 hotspot using TMS with Brainsight navigation (2) marking the coil center using cardboard replica of the coil (3) measuring $2 \mathrm{~cm}$ in the posterior direction from the marked M1 hotspot and marking the S1 hotspot.

The next stage in both studies was putting on the EEG cap and preparing the electrodes for the recording. In order to reduce the impedance between the electrode and the skin a special gel that is both abrasive and conductive was injected into the opening of the electrode with a syringe and then the skin was rubbed with a cotton swab through the electrode opening until the impedance was lower than $5 \mathrm{~K} \Omega$. Two skilled experimenters could prepare the subject in about 15 minutes. In less favorable circumstances the process could take 30-40 minutes, which is still considered relatively fast for a 64-channel system.

Following the reduction of the impedance we put the tracker glasses back on above the cap and verified that the registration was still good.

We then measured the resting motor threshold (RMT) which is defined as the stimulation intensity that elicits in the relaxed target muscle MEPs of at least $50 \mu \mathrm{V}$ in $50 \%$ of the trials (Rossini et al., 1994). The standard technique for finding the RMT is delivering a series of ten stimuli and looking for intensity for which in five out of ten trials a suprathreshold MEP is elicited. Usually with this method the series is 
continued until there are either more than 5 responses or more than 5 failures and then the intensity is reduced or increased respectively and the process is repeated. Although the process can be relatively fast when the experimenter is skilled, it is far from being very efficient and definitely does not utilize all the information one can learn from such a large number of stimuli. Awiszus (Awiszus, 2003) studied this issue in detail and his conclusion was that the variance of the "five out of ten" method estimate for the RMT is high and better methods exist using the same number of trials. He suggested a mathematically optimal procedure that he called "maximum likelihood threshold hunting" (MLTH). In this procedure TMS pulses are delivered at intensities computed by the MLTH routine so that the outcome of the stimulation would be the most informative for threshold determination at each stage. The outcome of each trial (success or failure to evoke a suprathreshold MEP) is provided to the routine as input. After several trials the routine produces an initial RMT estimate which improves with each subsequent trial. In most cases after about ten stimuli the routine starts to converge and the estimate does not change much any more. We used the implementation of MLTH published on the web by Awiszus himself. From our experience the procedure is robust and efficient. For the threshold measurement the coil was positioned at the hotspot using Brainsight navigation since the skin markings were covered at this stage by the cap. We always measured the threshold with the cap on since all the following applications of the TMS were with the cap. Thus our thresholds were always slightly higher than the standard RMTs of the same subjects with no cap.

After the threshold measurements the experiments of the two studies proceeded, according to each experiment's paradigm. In the MEP variability study the stimulation intensity was set at $115 \%$ RMT because at RMT there is by definition no response in $50 \%$ of the trials and we wanted to increase the number of trials we could analyze without saturating the response. We then proceeded by delivering a long series of TMS stimuli triggered by the experimenter.

In the PAS study we proceeded by recording the pre-PAS MN-SSEP followed by PAS intervention, post-PAS MN-SSEP recording and behavioral examination.

The study-specific details of the experimental procedures appear in the respective chapters. 
In each experiment (usually at the end) we performed electrode position measurement using the Brainsight system. One experimenter pointed at the fiducial points (nasion, preauricular points and inion) and the electrodes with the tracked points while another experimenter stored the pointer positions in the Brainsight software.

\subsection{Data analysis}

Since the theoretical basis of the data analysis appears in the introduction and the study specific details in the chapters below, this segment will describe only technical aspects of the analysis common to all studies.

Two main computational tools used for data analysis in the present thesis were Brain Electrical Source Analysis (BESA) software (MEGIS Software GmbH, Gräfelfing, Germany) and the open source Fieldtrip toolbox (http://www.ru.nl/fcdonders/fieldtrip/, F.C. Donders Centre for Cognitive Neuroimaging, Nijmegen, The Netherlands) running under Matlab programming environment (The MathWorks Inc., Natick, MA, USA).

BESA is a fully integrated EEG analysis environment that makes it possible to import raw data recorded on a variety of EEG systems, define epochs, perform artifact correction, rejection of artifact contaminated epochs and channels, digital filtering, averaging, frequency analysis and source analysis. The main weakness of BESA in the context of the present studies is absence of flexible tools for statistical analysis of the data.

Fieldtrip implements many functions duplicating those of BESA and also makes it possible to perform full analysis starting from raw data. In addition, it contains the recently developed CLUSTERRANDANALYSIS function implementing a variety of permutation based statistical analysis methods. The weaknesses of Fieldtrip important for the present studies are absence of graphical user interface for artifact rejection and source analysis. In addition the source analysis function of Fieldtrip is not well suited for sequential fitting of multiple sources and computing source activity based on complicated source models. 
Thus, the majority of analysis protocols in the present thesis combined BESA and Fieldtrip components. For this purpose a number of tools for interfacing BESA and Fieldtrip were developed in collaboration with Fieldtrip developer Robert Oostenveld. Some of these tools have been since integrated in standard Fieldtrip distribution.

Figure 13 shows in generalized way two possible protocols of this combined analysis. Figure Figure 13A shows analysis where BESA is only used for artifact rejection and most of the analysis is performed in Fieldtrip. The main purpose of BESA artifact rejection interface is providing the user with the possibility to balance the need for data preservation with the necessity to reject artifact contaminated epochs to improve the data quality. In addition, in some cases a few channels are contaminated heavily by artifacts (such as temporal muscle noise) but the majority of channels are clean. In these cases a small number of channels can be excluded from analysis to preserve more trials. In order to help the user make these decisions BESA computes 3 rejection criteria and presents them as a 2D image where the horizontal dimension corresponds to trials and vertical - to channels (Figure 14). The criteria are as follows:

1. Amplitude - the difference between the maximum and the minimum amplitude for a given trial and channel.

2. Gradient - the largest amplitude difference between two neighboring time samples within a trial for a given channel.

3. Low Signal - the variance of the gradient within a trial for a given channel. This criterion is used to detect channels that dropped out during the recording. In the present study low signal artifact did not occur so this criterion was not relevant. 


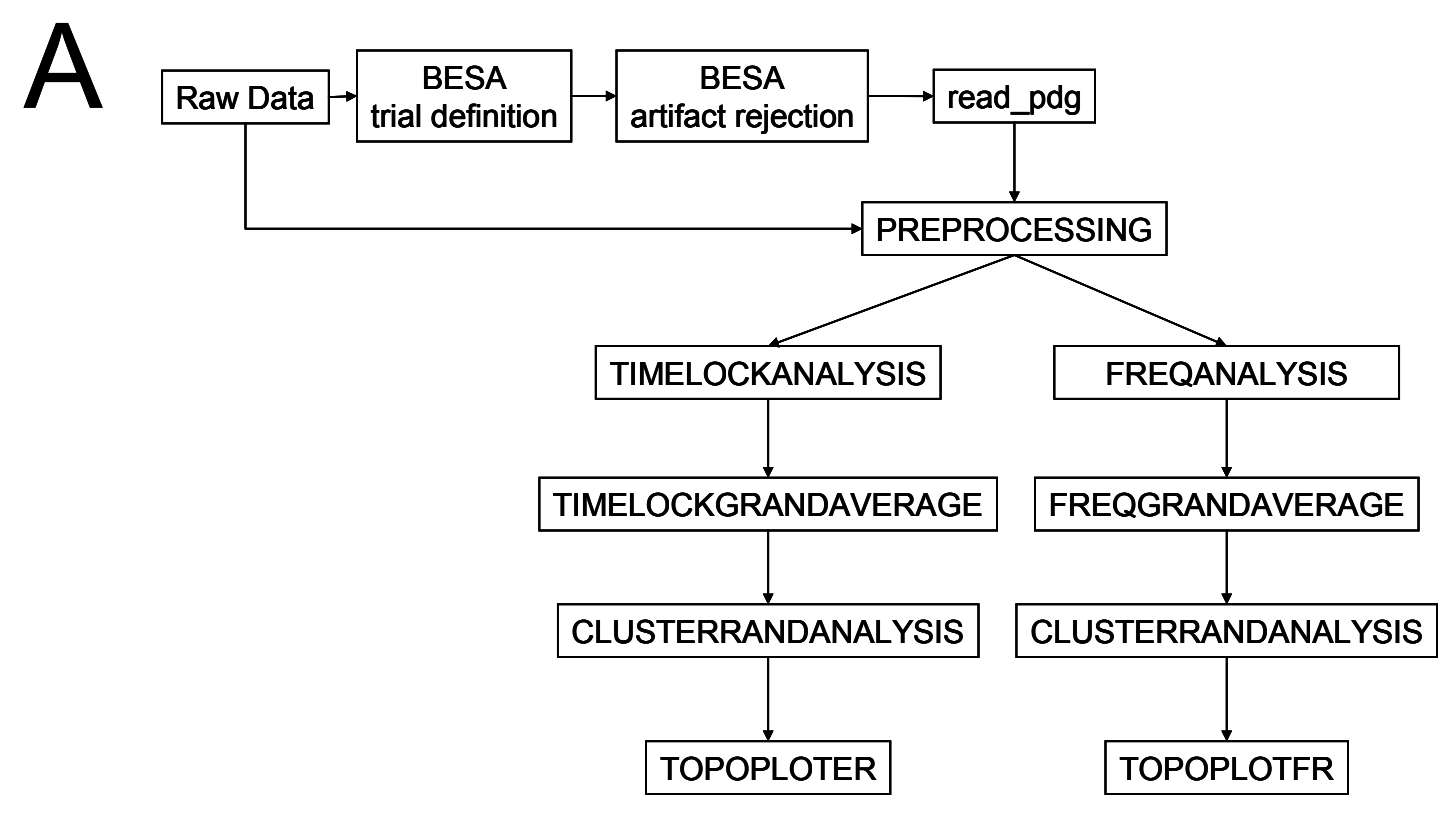

B

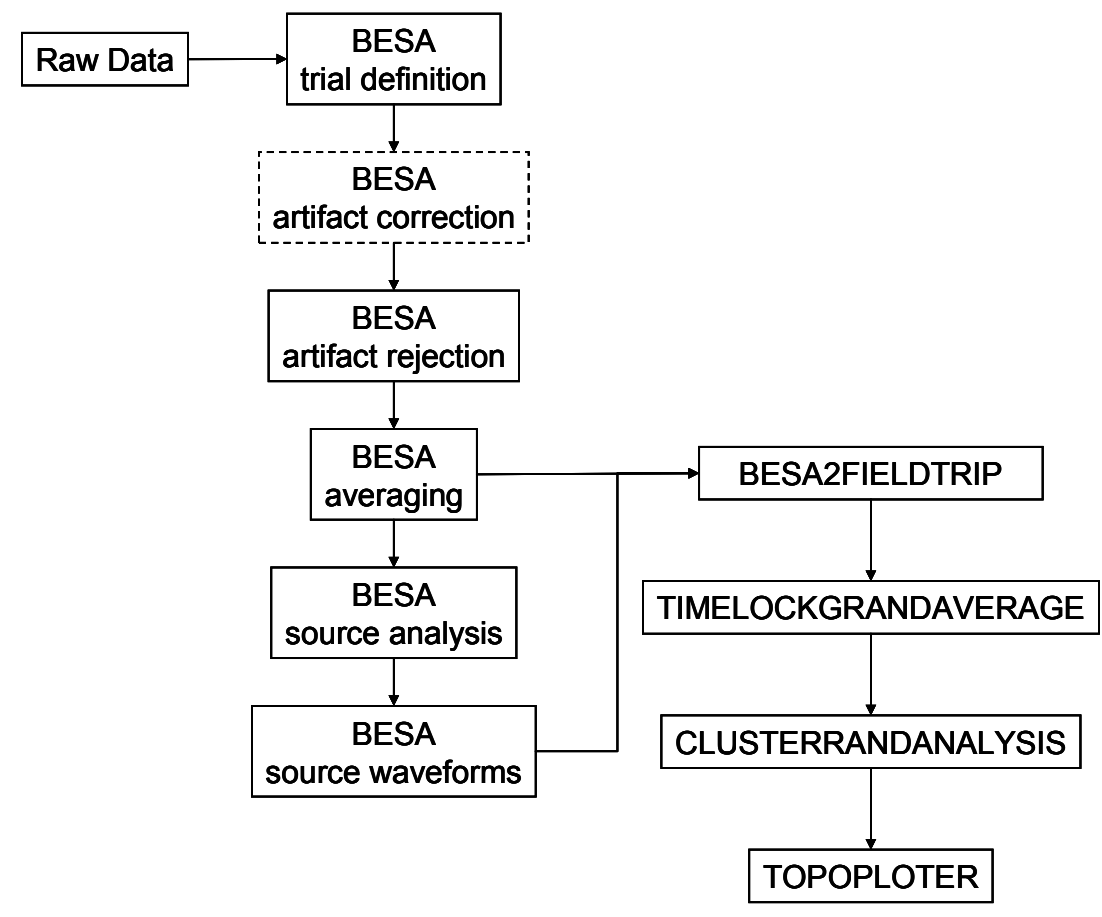

Figure 13. Generalized analysis flowcharts combining BESA and Fieldtrip. A. BESA is used only for preprocessing and further analysis is done in Fieldtrip. B. The analysis is done in BESA and Fieldtrip is used for assessment of statistical significance of the observed effects. 


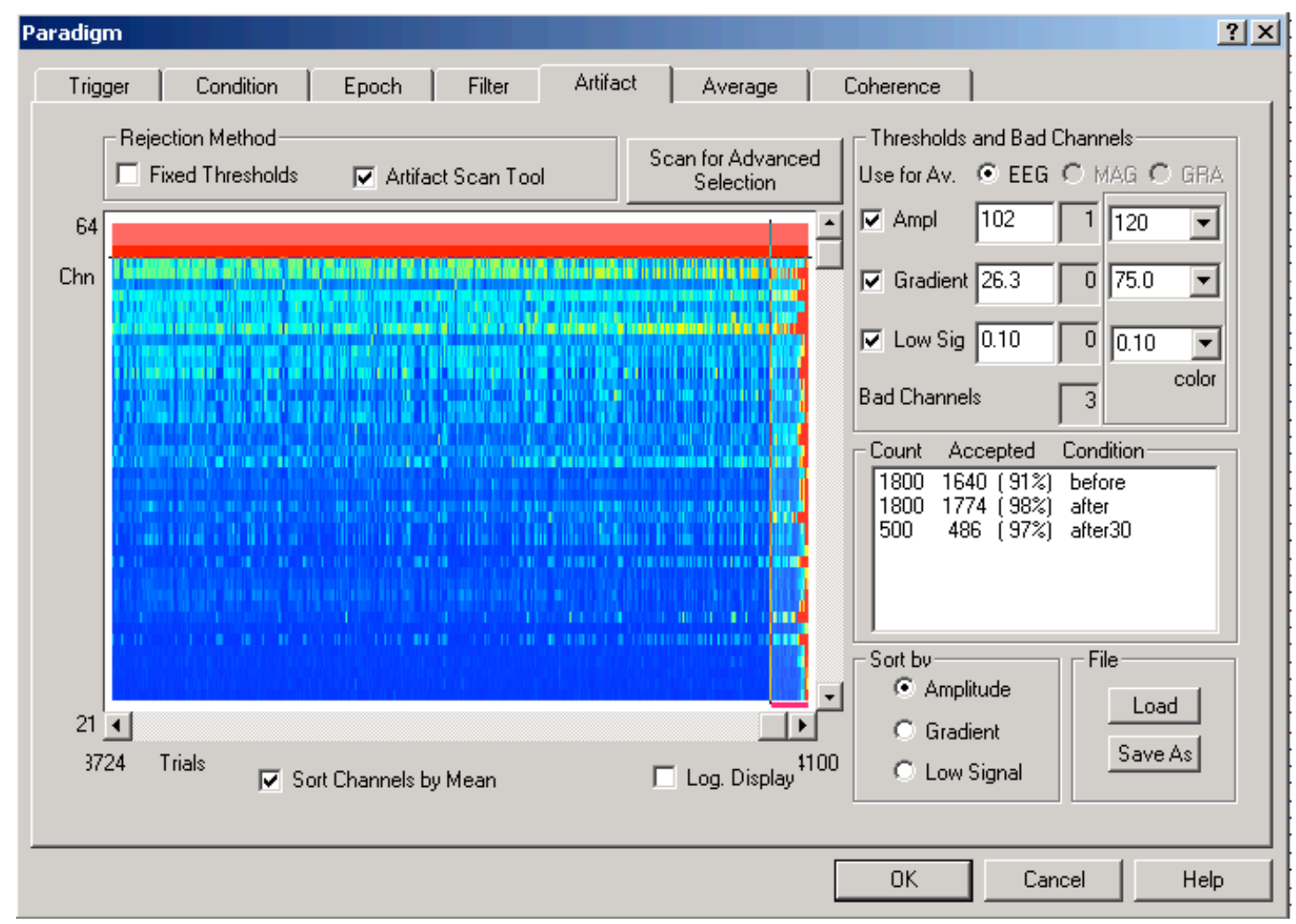

Figure 14. Artifact rejection interface of BESA. All the trials and channels are presented as a 2D image. The user can exclude channels (rows) or trials (columns) to balance the need for data preservation vs. the need to remove artifact contaminated trials.

The channels were sorted according to the level of artifact contamination from highest (up) to lowest (down) and the trials are also sorted in similar way from left (cleanest data) to right (most contaminated data). The user can then set rejection borders excluding either channels or trials or both. When a channel is excluded the trials are re-sorted to take this into account. The artifact rejection component generates an output file (PDG file) containing information about trial borders and trigger times and information about rejection criteria and user defined rejection borders. We developed a Matlab routine (read_pdg) that reads this file and produces a trial definition data structure with trials that were not rejected in BESA. This structure can be used as input to Fieldtrip PREPROCESSING function that reads the actual data from the raw data files. This data can be analyzed either in time domain or in frequency domain depending on the application. The statistical analysis can be performed with CLUSTERRANDANALYSIS and the results can be presented with Fieldtrip's graphical routines. 
An alternative analysis protocol uses more of BESA's capabilities by performing all the analysis stages up to ERP computation in BESA (Figure 13B). This protocol was used, for instance, in the study of EEG responses to TMS where TMS artifacts were corrected using correction algorithm implemented in BESA (Berg and Scherg, 1994a). Then artifact rejection was performed on corrected data so that the artifacts that could be corrected would not result in trial rejection. The corrected data was averaged and the resulting ERPs were exported and read into Fieldtrip using specially developed BESA2FIELDTRIP routine. In this case the Fieldtrip routines only arranged the data in the proper way to be used as input for CLUSTERRANDANALYSIS. A further extension of this protocol was performing source analysis of the average data in BESA that resulted in source activation waveforms. These waveforms could also be read into Fieldtrip and statistically analyzed there.

In summary, integrating BESA with Fieldtrip resulted in a powerful analysis platform combining the strengths of both tools. However, since the two software packages are undergoing continuous development, in the future each of them will probably contain all the tools necessary to perform all the steps of the analysis presented here. 


\section{Results}

\subsection{Artifact correction and source analysis of early electroencephalographic responses evoked by transcranial magnetic stimulation over primary motor cortex.}

\subsubsection{Introduction}

Transcranial magnetic stimulation (TMS) is a technique for noninvasive stimulation of the brain based on the principle of electromagnetic induction (Barker et al., 1985; Hallett, 2000). With the commonly used stimulation intensities and coils, the activated cortical area is several square centimeters. (Ruohonen and Ilmoniemi, 1999; Thielscher and Kammer, 2002).

The most prominent effect of a single TMS pulse is its ability to induce activity in contralateral muscles when applied over the primary motor cortex (M1). This has been the basis for use of TMS for diagnostics in neurology. When applied over the occipital visual areas TMS can induce visual sensations called phosphenes. However, these sensations are unreliable and have been used for research, but not for clinical diagnostics (Antal et al., 2004; Amedi et al., 2005). By applying a series of stimuli it is also possible to induce long-term effects which can be either suppression or facilitation of cortical excitability, depending on the exact stimulation protocol (Pascual-Leone et al., 1994; Chen et al., 1997; Wolters et al., 2003; Huang et al., 2005).

Combining TMS with neuroimaging methods such as positron emission tomography (PET), functional magnetic resonance imaging (fMRI) and electroencephalography (EEG) has the potential to expand the applicability of TMS for diagnostics and research since the effect of TMS application to any cortical area accessible to stimulation can be studied. Studies carried out until now with PET (Fox et al., 1997; Paus et al., 2001b; Fox et al., 2005) and fMRI (Bohning et al., 1999; Bohning et al., 2000; Baudewig et al., 2001; Nahas et al., 2001; Bestmann et al., 2004, 2005; Denslow et al., 2005) revealed TMS effects both at the stimulation site and at remote sites. However, the low temporal resolution of these methods did not allow definition of the order and the time course of activation of these areas. EEG which has superior time resolution can overcome this problem. However, analyzing event related 
potentials (ERPs) evoked by TMS has long been hampered by TMS induced artifacts. These slowly decaying transients or saturations in the recording did not allow examination of the time period immediately following the stimulus, which is the most interesting for studying functional connectivity. In order to overcome this problem EEG recording systems especially adapted for work with TMS had to be developed. Extensive research in this direction resulted in the development of sample-and-hold circuits capable of suppressing a large part of the TMS induced artifact (Virtanen et al., 1999) and allowing EEG recording as soon as $7 \mathrm{~ms}$ after the TMS pulse (or even 2 $\mathrm{ms}$ in the first study (Ilmoniemi et al., 1997)). Studies using this system were able to follow the propagation of the TMS induced activation to other ipsilateral and contralateral cortical areas (Ilmoniemi et al., 1997; Ilmoniemi et al., 1999; Komssi et al., 2002; Kahkonen et al., 2004; Kahkonen et al., 2005a) and examined the effect of stimulation intensity on TMS evoked potentials (Komssi et al., 2004; Kahkonen et al., 2005b).

Recently, using a commercial version of the same EEG system it was possible to show differences in functional connectivity between wakefulness and stages of deep sleep (Massimini et al., 2005a), to study the effects of TMS-induced long-term potentiation (LTP) in the motor cortex (Esser et al., 2006) and to characterize abnormalities in EEG responses to TMS in schizophrenic patients (Massimini et al., 2005b). These studies have shown the potential of combining TMS and EEG for the study of cortical function in humans.

However, the applicability of the method is presently limited since the artifact cannot be completely suppressed using specific hardware, especially when high stimulation intensities are used. This problem has been addressed in the previous studies by either excluding the early phase of the response (up to $20 \mathrm{~ms}$ and longer) from analysis (Paus et al., 2001a; Van Der Werf and Paus, 2006) or by excluding channels strongly affected by the artifact (Komssi et al., 2004; Kahkonen et al., 2005b). These approaches, however, do not allow recording responses in temporal or spatial proximity to the stimulus.

An alternative approach to the use of an EEG amplifier with a sample-and-hold circuit is offline correction of the stimulus artifact. This requires that the EEG responses are recorded using an amplifier with wide operating range that does not get saturated by 
the TMS pulse. Thut et al. described a method where artifact is removed by subtracting an ERP induced by TMS alone from an ERP induced by TMS during a task (Thut et al., 2003). This approach is suitable for studying the effect of TMS on potentials related to processing of sensory stimuli and to performance of experimental tasks. Its advantage is that not only the TMS pulse artifact but all other TMS effects not related to the task, such as the auditory and somatosensory responses are eliminated. However, it does not allow studying the responses evoked by TMS itself since these responses are eliminated by the subtraction procedure (Thut et al., 2005). In some cases when only late components of the ERP are of interest, the parts of the recording affected by the artifact can be excised from the recording in order to prevent their interference with digital filtering and the remaining part of the ERP can be analyzed by standard methods. Obvioulsy, this method is limited by its inability to examine the early part of the response that can be as long as $40 \mathrm{~ms}$ (Fuggetta et al., 2006).

In the present study we used a methodology originally developed for correcting eye movement artifacts based on their scalp topography (Berg and Scherg, 1994a; Ille et al., 2002) in order to correct the residual TMS artifacts. This method made it possible to substantially reduce the contamination by artifacts of the early TMS evoked ERPs without distorting the physiological components of the response.

The goal of this study was to apply this multiple source artifact correction method to previously published data (Komssi et al., 2004) in order to develop a multiple source model of the early EEG responses to magnetic stimulation of the left and the right motor cortex. The early part of the response, up to $60 \mathrm{~ms}$ after the magnetic stimulus, is most severely affected by the TMS artifact and, therefore, requires special correction, unlike the late part of the response which can be analyzed similarly to other kinds of evoked responses (Fuggetta et al., 2006). 


\subsubsection{Methods}

\subsubsection{Experimental procedures}

Detailed description of the experimental procedures can be found in the original publication (Komssi et al., 2004; Kahkonen et al., 2005b).

A total of 9 healthy volunteers ( 2 female, 2 left handed, age 27 \pm 4 ) participated in the two studies after giving a written informed consent. The experimental protocol was approved by the ethical Committee of the Department of Radiology, Helsinki University Central Hospital.

Biphasic magnetic pulses (duration $385 \mu \mathrm{s}$ ) were delivered with a custom-made figure-of-eight coil consisting of two coplanar circular loops $(40 \mathrm{~mm} \varnothing)$ so that the induced current was in the posterior-anterior direction. The electromyogram was recorded from the right abductor digiti minimi (ADM) muscle. The optimal position for stimulation of the ADM was found separately in each hemisphere. The resting motor threshold (RMT) was determined by adjusting the TMS intensity until $50 \mu \mathrm{V}$ motor evoked potentials were evoked in about $50 \%$ of the trials. During stimulation, the subject sat in a reclining chair with the neck and back supported by pillows, arms relaxed and eyes closed, wearing ear plugs.

TMS was delivered to the left M1 and middle frontal gyrus in sequences of 50 pulses with an interstimulus interval of 1.5-2.5 s at constant intensity. Four sequences, with intensities of $60,80,100$, and $120 \%$ of RMT, were targeted in random order to one or two stimulation sites. This was repeated after a 5-10 minutes break. In 7 subjects left and right M1 were stimulated in the same experiment; in 2 subjects left M1 and left PFC were stimulated. Thus, in total 9 cases of left M1 stimulation, 7 cases of right M1 stimulation were included in the present study.

The EEG was recorded from 60 scalp electrodes (small $\mathrm{Ag} / \mathrm{AgCl} \mathrm{C}$-shaped) referred to the forehead. The saturation of the EEG amplifiers by the TMS pulse was avoided by using a sample-and-hold circuit (Virtanen et al., 1999). The data were sampled at the rate of $1450 \mathrm{~Hz}$ and digitally low-pass filtered at $250 \mathrm{~Hz}$. 
The data subjected to further analysis consisted mostly of concatenated epochs $(-100$ to $+300 \mathrm{~ms}$ relative to the stimulus). Continuous data was available only for two of the subjects.

\subsubsection{Analysis of the EEG data}

Initial analysis of the data used for the present study has already been published (Komssi et al., 2004; Kahkonen et al., 2005b). The analysis presented here improves the previously presented analysis methods in three main respects.

1. We applied the artifact correction methodology previously suggested for common EEG artifacts such as eye blinks (Berg and Scherg, 1994a) to the residual TMS artifact and were able to show that in combination with the hardware artifact blocking circuit (Virtanen et al., 1999) this methodology makes it possible to remove the artifact to a very large extent at all stimulation intensities.

2. Based on artifact corrected data, we suggest a source model for generation of TMS evoked potentials. This model allowed us to separate different components of the response and estimate propagation delays of TMS evoked activation for different brain areas.

3. We applied a novel framework for statistical analysis of neuroimaging data in order to assess the significance of the effect of TMS intensity on the amplitude of source activity.

Thus, our analysis procedure consisted of 3 stages: correction of the TMS induced artifact, computing the cortical activity from corrected EEG data using source modeling and, finally, statistical analysis of the brain source activity estimated for each subject and condition. 


\subsubsection{Correction of the artifact}

The method used for artifact correction in the present study was developed by Berg and Scherg (Berg and Scherg, 1994a). It is implemented in the BESA software (MEGIS Software GmbH, Gräfelfing, Germany) that was used for pre-processing of the data.

Under the assumption of linear summation, the relationship between the scalp voltage and the activity of the underlying sources can be written as follows:

$$
V_{n \times t}=L_{n \times m} \cdot S_{m \times t}
$$

$V_{n \times t}$ is the matrix of observed voltages ( $\mathrm{n}$ - number of channels, $\mathrm{t}$ - number of time frames). Each row of $V_{n \times t}$ is a waveform recorded by one of the electrodes and each column is a voltage map on the scalp at a specific time point.

$S_{m \times t}$ is the matrix of source activities, where each row is a source waveform. $\mathrm{m}$ is the number of sources.

A specific case which is of interest in the present study is the use of discrete multiple source modeling for correcting artifacts. There are artifacts present in practically any EEG recording, such as eye movements, blinks and muscle artifacts and this study deals specifically with artifacts generated by TMS. Under the assumption of linear summation, artifacts can be presented as part of the sources in matrix S.

$$
V_{n \times t}=\left(\begin{array}{cccccc}
l_{1}^{a_{1}} & \mathrm{~L} & l_{1}^{a_{r}} & l_{1}^{b_{1}} & \mathrm{~L} & l_{1}^{b_{p}} \\
\mathrm{M} & 0 & \mathrm{M} & \mathrm{M} & \mathrm{O} & \mathrm{M} \\
l_{n}^{a_{1}} & \mathrm{~L} & l_{n}^{a_{r}} & l_{n}^{b_{1}} & \mathrm{~L} & l_{n}^{b_{p}}
\end{array}\right) \cdot\left(\begin{array}{ccc}
s_{1}^{a_{1}} & \mathrm{~L} & s_{t}^{a_{1}} \\
\mathrm{M} & 0 & \mathrm{M} \\
s_{1}^{a_{r}} & \mathrm{~L} & s_{t}^{a_{r}} \\
s_{1}^{b_{1}} & \mathrm{~L} & s_{t}^{b_{1}} \\
\mathrm{M} & 0 & \mathrm{M} \\
s_{1}^{b_{p}} & \mathrm{~L} & s_{t}^{b_{p}}
\end{array}\right)=L_{n \times r}^{a} \cdot S_{r \times t}^{a}+L_{n \times p}^{b} \cdot S_{p \times t}^{b}
$$

In this case the topography of the artifacts is approximated by $\mathrm{r}$ components and the brain activity is represented by a model with $\mathrm{p}$ sources. The equation can be solved by inverting the combined leadfield matrix L with the appropriate mathematical method 
(in BESA Moore-Penrose pseudoinverse is computed with Tikhonov regularization). Note that the rows of the inverse matrices related to the artifact topographies are dependent on the brain source topographies and vice versa. Therefore, the quality of separation between brain activity and the artifacts depends on both the model of the artifact and the model of the brain activity. This poses a problem since an adequate model of the brain activity cannot be built in the presence of the artifact, whereas the artifact cannot be corrected well without such a model. In studies where the generators of evoked potentials in question are well known, standard models based on previous studies can approximate the brain activity rather well (Scherg et al., 2002). Since the present study is to our knowledge the first attempt to suggest a detailed model of the potentials evoked by TMS, we had to base our artifact correction on an iterative approach consisting of two stages. In the first stage the brain activity was represented by a generic 'Brain Regions' model consisting of 15 regional sources (Scherg and Von Cramon, 1986) covering all cortical regions and capable of reasonably approximating any activity generated in the cerebral cortex (Scherg et al., 2002). After artifact correction based on this model, we used the grand average of the corrected data to construct a new model specifically accounting for the TMS evoked activity. In the second stage, the activity of sources of the new model was recomputed by inverting the new lead field matrix consisting of the lead fields of the sources in the new model and the previously estimated artifact topographies.

The main differences between modeling artifacts and brain sources are that we do not expect artifacts to be well modeled by equivalent current dipoles (ECD). Under these conditions it is not necessary to have a physical model of artifact generation, but it is sufficient to estimate the columns in matrix L representing the effect of the artifact on the electrodes. This can be done by performing principal component analysis (PCA) of an artifact dominated time segment (Ille et al., 2002). PCA is a linear transformation that chooses a new coordinate system for the data set such that the greatest variance is accounted for by the first axis (also called the first principal component). Then the second axis orthogonal to the first is chosen to account for the greatest remaining variance, and so on. The idea is that in many cases although the dimensionality of the raw data is high (in the case of EEG equivalent to/given by the number of electrodes) the number of underlying sources and artifact topographies is low. 
The analysis stages were as follows (flowchart of the analysis is shown in Figure 15):

1. The head channels that were obviously disconnected during the recording or severely contaminated by noise were identified by eye and excluded from further analysis (see also (5) below).

2. For most subjects the epoched data did not contain eye blinks since eye-blink contaminated trials were excluded in the course of the original analysis. In cases where eye blinks were present, they were identified using the automatic search and average procedure of the BESA program. A topography of the eye blink artifact was then estimated based on these averages and used in combination with TMS artifact topographies (see below) for artifact correction.

3. TMS evoked ERPs were computed by averaging the data time-locked to the TMS triggers (baseline corrected with baseline -100 to $0 \mathrm{~ms}$ ). The segments following the stimulus were examined for the presence of TMS artifacts defined as sharp peaks that clearly exceeded normal ERP amplitudes (i.e. $>30 \mu \mathrm{V}$ ). The topographies of the artifact were estimated by performing PCA of a segment around these peaks. The data were then corrected using the defined artifact topographies with the method described above. At this stage the generic 'Brain Regions' model (Scherg and Von Cramon, 1986) was used for artifact correction. The corrected data was again averaged around triggers and the correction was repeated until there were no more clear artifacts in the average. For experiments in which more than one location was stimulated and the coil was moved between the two repetitions of stimulating the same location, we found that the artifact topography differed between the two repetitions. We therefore applied our correction procedure separately for each repetition in these cases. In total, 128 data files were corrected separately. In 2 cases no correction was necessary since no clear artifacts were found. In 98 cases a single topography was sufficient to correct the artifact. In 19 cases two topographies were used, in 7 cases - three topographies and five and six topographies were necessary in a single case each. These numbers include both TMS artifact and eye blink artifact topographies.

4. The corrected data computed by subtracting the data accounted for by the artifact in the presence of the 'Brain Regions' model was then examined for other artifacts and trials still contaminated after correction were rejected using the BESA artifact rejection interface (Scherg et al., 2004). Trials were considered artifact and excluded from averaging if they satisfied one of the following two conditions: (1) The amplitude difference between the largest and the smallest sample within the 
trial in any channel exceeded the maximal amplitude threshold. (2) The voltage difference between two adjacent samples in any channel exceeded the maximal gradient threshold. The thresholds were adjusted individually using graphical user interface to balance between the need to retain trials for analysis and the need to reject artifact contaminated trials. The maximal amplitude threshold was $207.0 \pm 48.1 \mu \mathrm{V}$. The maximal gradient threshold was $82.5 \pm 27.1 \mu \mathrm{V}$. In some cases, additional channels were excluded from analysis at this stage in order to retain more trials (see also (5) below).

5. Average evoked responses were computed for each data file twice - with and without artifact correction. The two averages were compared in order to verify that only the artifact was eliminated and the activity at later times and at other scalp areas was not affected. In some cases channels were excluded at this stage if the activity after correction was clearly distorted and not consistent with activity at the neighboring channels. The total number of channels excluded from analysis at stages 1, 4 and 5 (averaged across all analyzed files) was 4 \pm 3 , max 13 .

6. For each subject, intensity and location an average was computed over the two repetitions of stimulating with the same parameters. Prior to this step, excluded channels were interpolated using spherical spline interpolation (Scherg et al., 2002).

7. From these data grand averages over subjects were computed for each location and each intensity. For the creation of a grand average source model, one common grand average was computed over the intensities of 80, 100 and $120 \%$ RMT, separately for right and left motor TMS. 


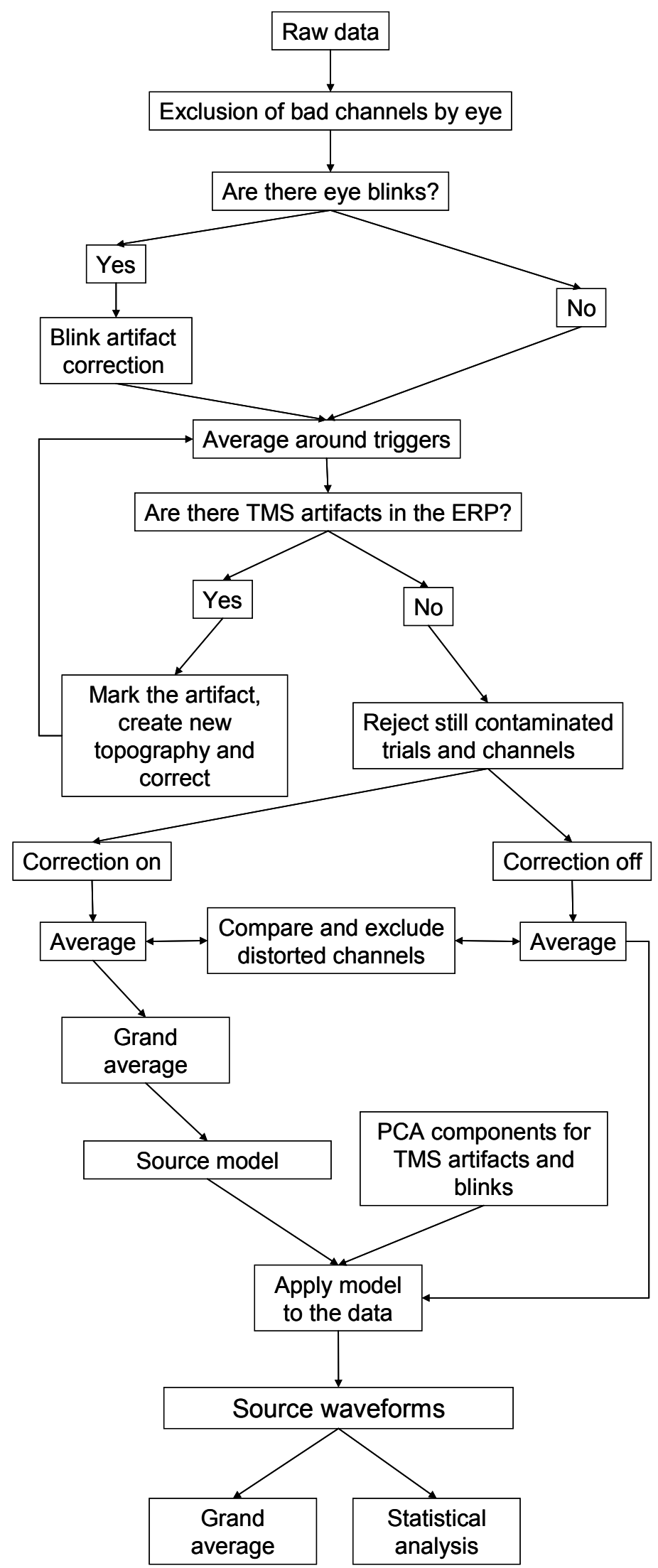

Figure 15. Flowchart for artifact correction and analysis of TMS-evoked ERPs 


\subsubsection{Modeling the responses evoked by TMS}

The grand averages across all subjects and intensities from 80 to 120\% were analyzed using the sequential multiple source analysis strategy (Scherg and Berg, 1996). In order to remove low frequency activities, the data were filtered with a forward $5-\mathrm{Hz}$ high-pass filter for source modeling. First, a regional source was fitted to the earliest epoch free of residual artifact (11-20 ms). The regional source was oriented and the predominant dipole retained as the first source of the multiple source model. Then, symmetric pairs of regional source were added to the model in a sequential procedure to account for the residual activities in the next baseline to peak interval. Again, after each pair of regional sources was added and fitted, the sources were rotated at their earliest peak and converted into single dipoles retaining the predominant orientation. The right and left TMS data were fitted in alteration. This showed a fair symmetry of the sources with some of the ipsilateral activities matched by contralateral activities. Since the contralateral activities were weak and showed a similar orientation as the ipsilateral activity when stimulating the other side, the symmetry constraint was released and the ipsilateral dipoles were refitted in their respective intervals and the ipsilateral stimulation conditions. Thus, a grand average model that contained a comparable ipsi- and contralateral source to each stimulation condition could be created while the fitting optimization was only performed on the ipsilateral, i.e. stronger source activities. Thereby, the uncertainty of fitting a small contralateral component with unstable location was overcome. The final common grand average model with 9 fixed dipoles could then be used to construct a fixed source montage, i.e. an inverse spatial filter (Scherg et al., 2002; Hoechstetter et al., 2004), that allowed decomposition the data of each subject and each condition into source waveforms for statistical analysis. This set of dipoles consisted of three symmetric pairs of dipoles: anterior motor (AM), midline-frontal-motor (MFM) and temporoparietal junction (TPJ) and three dipoles in the cerebellum $(\mathrm{Cb})$. The Talairach (Talairach and Tournoux, 1988) coordinates of model sources and the brain areas in their vicinity are given in Table 2 .

\subsubsection{Computing the activity of the model sources}

In the next step of the analysis the models were applied to the data. This was done for each original data file separately. The general model described above was combined 
with the specific artifact topographies of each data segment and then source waveforms for model sources were saved. Averages were subsequently computed between the two files of the same condition, same subject and also grand averages of the same condition across subjects. No filtering was applied to the source waveforms prior to statistical analysis. The waveforms were baseline corrected by baseline correction of the raw ERPs.

\subsubsection{Statistical analysis}

The source waveforms were analyzed statistically using the Fieldtrip open source Matlab toolbox (http://www.ru.nl/fcdonders/fieldtrip/) developed at the F.C. Donders Centre for Cognitive Neuroimaging. The nonparametric statistical procedure implemented in this toolbox allows assessing the significance of spatio-temporal scalp patterns (Maris and Oostenveld, 2006). This solves the problem of multiple comparisons that would arise if t-tests were performed on multiple time-frames.

The analysis used in the present study was regression analysis for finding temporal patterns significantly dependent on TMS intensity. Since our data consists of repeated measurements from the same subjects, classic linear regression would not be appropriate. Our analysis is thus based on dependent samples regression statistic suggested by Lorch and Myers (Lorch and Myers, 1990). This statistic is computed as follows: in the first stage the regression coefficient between the independent variable $\mathrm{X}$ and dependent variable $\mathrm{Y}$ of interest (in our case the TMS intensity and the neural response respectively) is determined separately for each subject.

$$
\hat{\beta}_{s}=\frac{\sum_{i=1}^{K}\left(Y_{i}-\bar{Y}\right)\left(X_{i}-\bar{X}\right)}{\sum_{i=1}^{K}\left(X_{i}-\bar{X}\right)^{2}}
$$

where $\mathrm{K}$ - number of samples for each subject (in our case $\mathrm{K}=4$ because four TMS intensities were tested in all experiments). 
In the second stage the independent regression $\mathrm{t}$ statistic is computed from the individual regression coefficients by dividing the mean regression coefficient by its standard error across subjects:

$$
T_{d s r}=\frac{\bar{\beta}}{S E(\beta)}
$$

In order to find the aspects of the response that are significantly dependent on TMS intensity, this statistic can be tested against a t-distribution with $\mathrm{N}-1$ degrees of freedom $(\mathrm{N}-$ number of subjects).

Since in total 12 sets of waveforms were analyzed for the two sides a problem of false positives arises due to multiple comparisons. This problem can be solved within the nonparametric analysis framework by running analysis on all the waveforms simultaneously. This, however, reduces the power of the test, especially if the magnitudes of the effect differ between different sources, as is likely to be the case. We therefore, performed the analysis both individually for each source and for all the waveforms. The temporal clusters displaying dependence on TMS intensity are the same in both cases and only their p-values were affected. 


\subsubsection{Results}

\subsubsection{Correction of the residual artifact}

Figure 16 shows the grand average of responses to TMS at the left M1 before (A) and after (B) artifact correction. In the uncorrected data sharp peaks can be seen around the stimulation site, exceeding by far the magnitude typical of evoked potentials, as evident from their comparison with later peaks. The recording returns to baseline only about $30 \mathrm{~ms}$ after the stimulus. The residual artifact, therefore, completely masks the early ERP response. After correction the sharp peaks are completely removed. The correction, however, does not affect the later activity in which there is similarity between corrected and uncorrected waveforms. In some cases, the stimulation induced a DC shift at electrodes close to the coil, perhaps due to mechanical movement of the electrode. These shifts are also corrected by our correction procedure. 

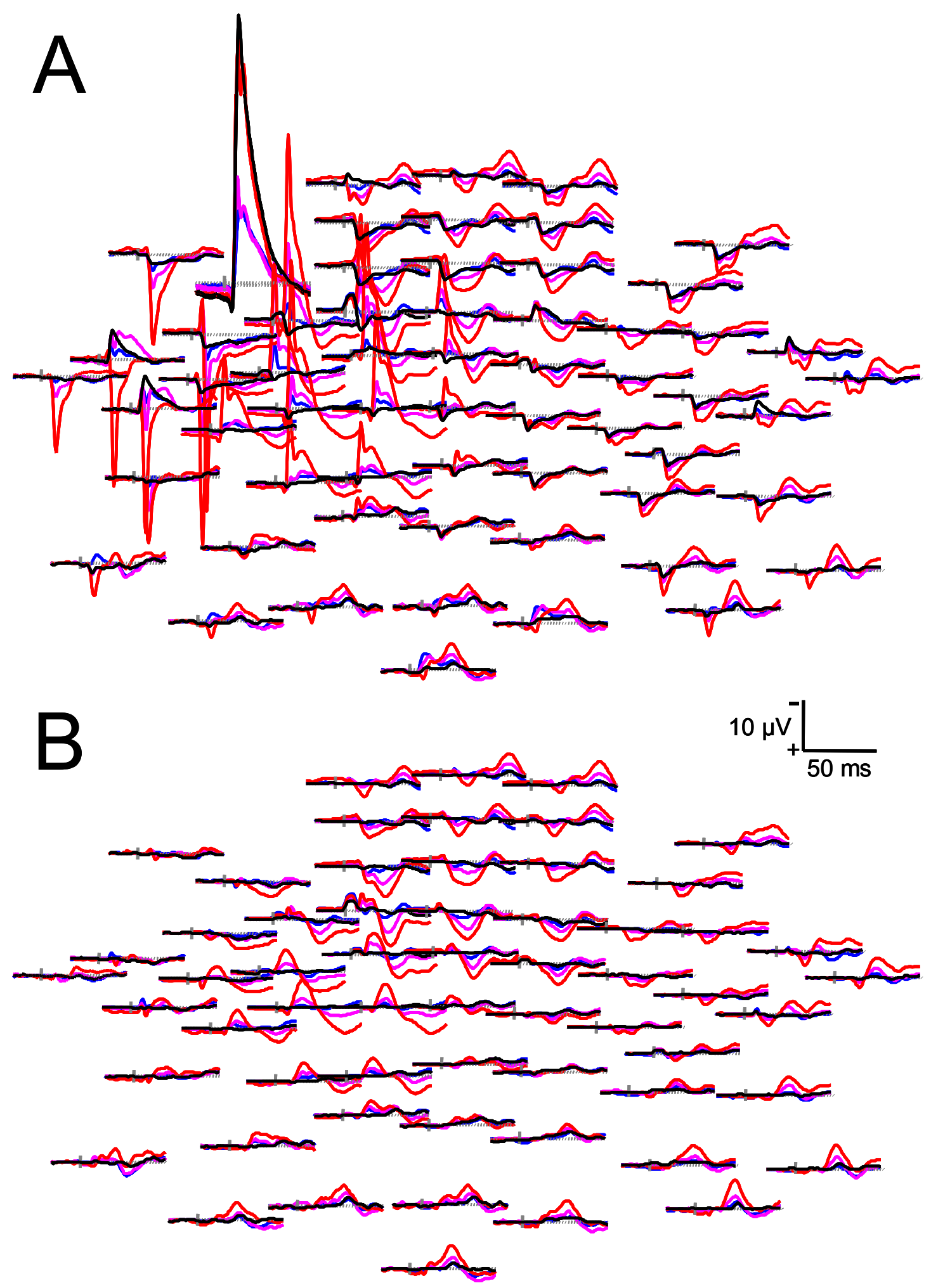

Figure 16. Grand average of TMS-evoked ERPs before (A) and after (B) artifact correction. TMS was applied at the left M1. The results are shown for 4 stimulus intensities $60 \%$ (black), $80 \%$ (blue), $100 \%$ (magenta) and $120 \%$ (red) RMT. 


\subsubsection{Prominent scalp distribution patterns observed in the corrected data}

To reveal the most robust features of the response and reduce the noise, the responses to TMS at $80 \%, 100 \%$ and $120 \%$ RMT were averaged at the initial stage of the analysis and the grand average of these averaged responses was examined. Figure 17 shows 'butterfly plots' of the grand averaged responses to stimulation of left and right M1. Scalp potential distribution at the prominent response peaks is also shown. Note the remarkable symmetry between the response scalp maps at closely corresponding times between the left and right M1 stimulation. The earliest pattern observed immediately following the amplifier reconnection and the associated artifacts is negativity ipsilateral to the stimulation around $10-12 \mathrm{~ms}$ after stimulus. This pattern is followed by a dipolar pattern at the side of the stimulation with frontal positivity reaching to the side contralateral to the stimulation and a posterior negativity (15$25 \mathrm{~ms})$. The next pattern is a widespread anterior-central positivity lateralized to the stimulation side $(25-35 \mathrm{~ms})$. It is followed at $35-50 \mathrm{~ms}$ by a dipolar pattern similar to that of $15-25 \mathrm{~ms}$ with the opposite polarity. This sequence of scalp patterns has been described in the original publication of Komssi et al. (Komssi et al., 2004) and recently independently confirmed by Bonato et al. (Bonato et al., 2006) using an fMRI compatible EEG system in which the TMS artifact is relatively brief. In addition, a pattern not previously described was observed at the back of the scalp around $30 \mathrm{~ms}$ after the stimulus. This pattern consisted of a positivity lateralized to the stimulation side and a negativity at the occipital electrodes slightly lateralized to the side contralateral to stimulation. 


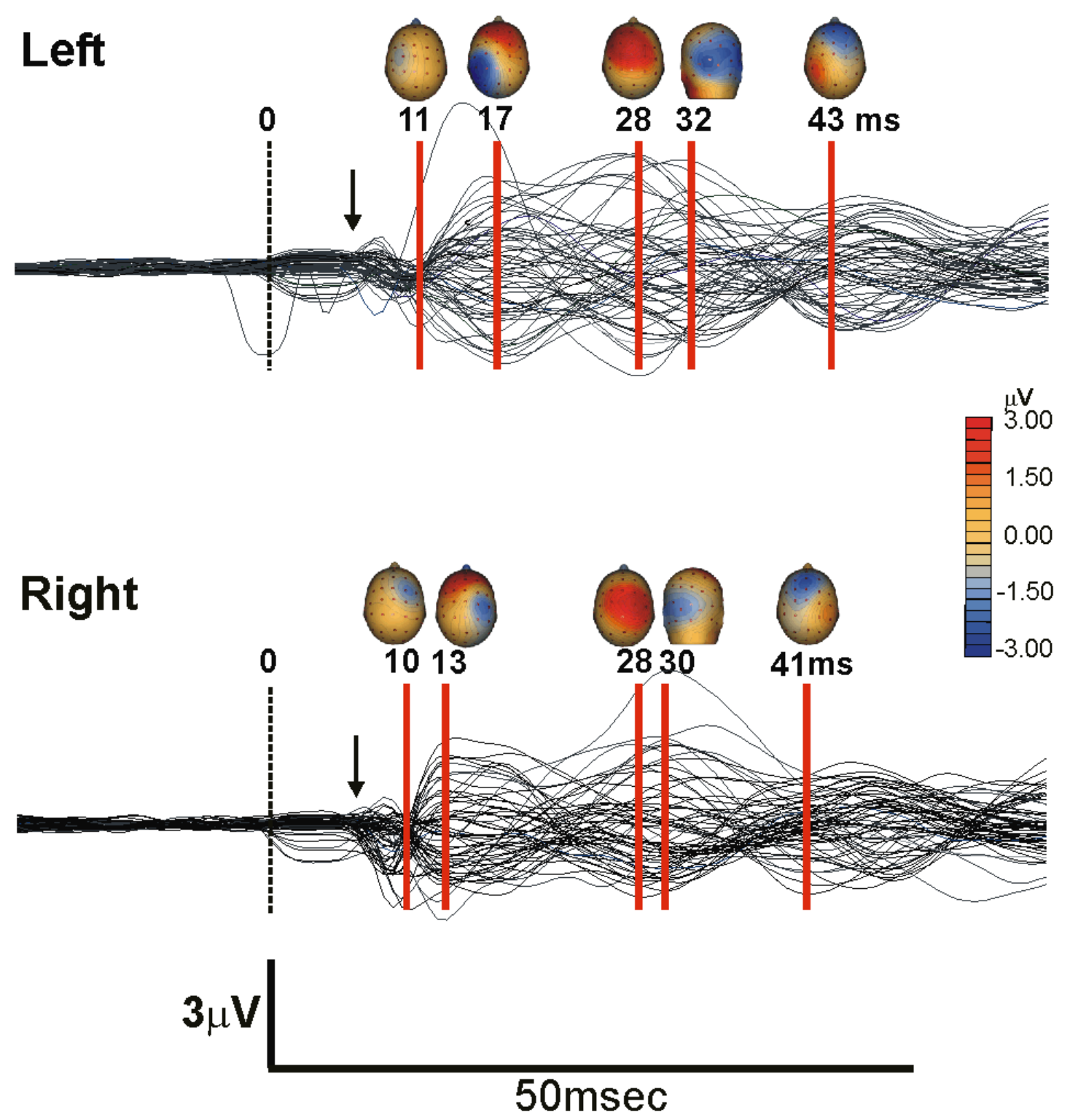

Figure 17. Prominent scalp potential patterns in the artifact corrected data. The data were averaged across $80 \%, 100 \%$ and $120 \%$ intensities and grand averaged across subjects. The arrow marks the end of the time window where the amplifiers were disconnected. Note the symmetry between the responses to left and right M1 stimulation. 


\subsubsection{Source model reveals similar response sequence for left and right M1 stimulation}

Figure 18 shows the source waveforms and scalp projections of equivalent current dipoles of the model fitted as described in Methods for the grand average of combined responses for $80 \%, 100 \%$ and $120 \%$ RMT. The names, locations and orientations of model sources are shown in table 2. To facilitate the comparison between left and right $\mathrm{M} 1$ stimulation we use in the following discussion the suffixes $-\mathrm{i},-\mathrm{c}$ and $-\mathrm{m}$ meaning ipsilateral and contralateral to the stimulation and mid-line, respectively. Clear symmetry can be observed between the responses to the left and right M1 stimulation. In addition, the model reveals a likely sequence of signal propagation following magnetic stimulation of M1. The sequence is AMi (peaking at $17 \mathrm{~ms}$ for left, $13 \mathrm{~ms}$ for right stimulation), MFMi (negative peak at $17 \mathrm{~ms}-1 \mathrm{eft}, 12 \mathrm{~ms}-$ right, positive peak 28ms-left , 22ms - right), MFMc which is weakly active around the same time as MFMi, Cbi (32ms left, $30 \mathrm{~ms}$ - right), TPJi (37ms left, 34ms right) and Cbm (56ms left, $51 \mathrm{~ms}$ right). 


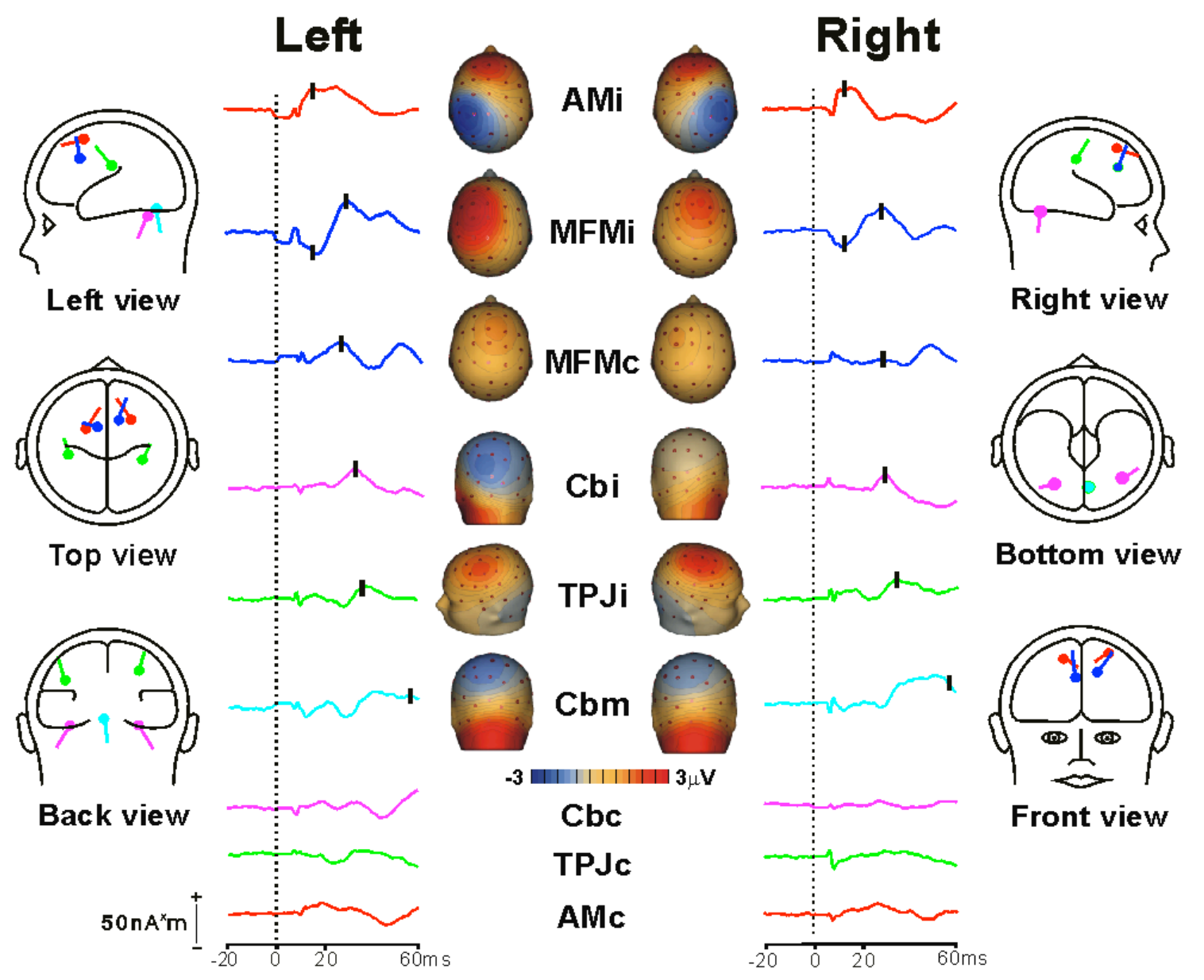

Figure 18. The multiple source model fitted to the grand averaged data and the corresponding source waveforms. The side panels show the model dipoles from different view angles (AC-red, MFM-blue, TPJ-green, Cb - magenta (left, right), cyan (mid-line)). In the middle the source waveforms separately for left and right $M 1$ stimulation and scalp projections for the sources for which clear peaks were observed. The black bars mark peaks mentioned in the text. The magnitude of the scalp potentials shown in the scalp maps corresponds to the marked times (the second peak for MFM). Note the similarity of the scalp projections to raw data scalp patterns of Figure 17. 
Table 2. The model sources fitted to TMS evoked ERPs (Talairach coordinates)

\begin{tabular}{|c|c|c|c|c|c|c|c|c|}
\hline \multirow{2}{*}{ Source name } & \multirow{2}{*}{ Side } & \multicolumn{3}{|c|}{ Location } & \multicolumn{3}{|c|}{ Orientation } & \multirow{2}{*}{$\begin{array}{l}\text { Brain areas } \\
\text { within } 1 \mathrm{~cm} \\
\text { radius }\end{array}$} \\
\hline & & $\mathbf{X}$ & $\mathbf{Y}$ & $\mathbf{Z}$ & $\mathbf{X}$ & $\mathbf{Y}$ & $\mathbf{Z}$ & \\
\hline \multirow{2}{*}{$\begin{array}{l}\text { Anterior } \\
\text { Motor } \\
\text { (AM) }\end{array}$} & Left & -24.5 & 7.8 & 60.3 & 0.4 & 0.9 & -0.2 & BA 6 \\
\hline & Right & 19.7 & 20.6 & 54.8 & -0.4 & 0.9 & -0.2 & BA 6,8 \\
\hline \multirow{2}{*}{$\begin{array}{l}\text { Midline } \\
\text { Frontal } \\
\text { Motor } \\
(\mathrm{MFM})\end{array}$} & Left & -12.4 & 14.5 & 40.2 & -0.5 & 0.1 & 0.9 & BA 32 \\
\hline & Right & 8.5 & 23.8 & 34.1 & 0.1 & 0.2 & 1.0 & BA $32,6,8,9,24$ \\
\hline \multirow{3}{*}{$\begin{array}{l}\text { Cerebellum } \\
\text { (Cb) }\end{array}$} & Left & -36.9 & -57.7 & -27.3 & -0.4 & 0.5 & -0.8 & Left cerebellum \\
\hline & Middle & -3.7 & -69.2 & -21.7 & 0.1 & 0.0 & -1.0 & $\begin{array}{l}\text { Left cerebellum, } \\
\text { right cerebellum }\end{array}$ \\
\hline & Right & 30.1 & -63.6 & -28.0 & 0.4 & 0.0 & -0.9 & Right cerebellum \\
\hline \multirow{2}{*}{$\begin{array}{c}\text { Temporo- } \\
\text { Parietal } \\
\text { Junction } \\
\text { (TPJ) }\end{array}$} & Left & -42.2 & -22.1 & 28.8 & -0.2 & 0.5 & 0.8 & BA 2 \\
\hline & Right & 31.0 & -29.1 & 38.0 & 0.2 & 0.4 & 0.9 & BA $2,3,40$ \\
\hline
\end{tabular}

\subsubsection{Dependence of the source waveforms on stimulation intensity}

To analyze the TMS intensity dependence of the response of each of the model sources, non-parametric statistical analysis of the source waveforms was performed using dependent samples regression statistic (see Methods). The results of the analysis are shown in Figure 19. The sources included in the analysis were the six sources showing clear activation for each side (Figure 18). Only the MFMi sources showed a significant TMS dependency after multiple comparisons correction (26-43ms $\mathrm{p}<0.01$ ( $p<0.02$ corrected) left, 28-39ms $p<0.01$ ( $p<0.05$ corrected) right). Without the correction, intensity dependent temporal clusters were also observed for AMi (48-55 ms $\mathrm{p}<0.05$ left, $45-56 \mathrm{~ms} \mathrm{p}<0.02$ right), Cbi (12-20 ms $\mathrm{p}<0.02$ left, $48-54 \mathrm{~ms} \mathrm{p}<0.05$ right) and $\mathrm{Cbm}(39-45 \mathrm{~ms} \mathrm{p}<0.05$ left, $41-52 \mathrm{~ms} \mathrm{p}<0.01$ right $)$. 


\section{Left}
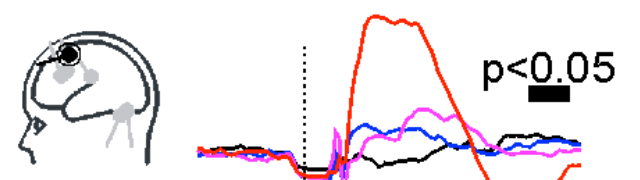

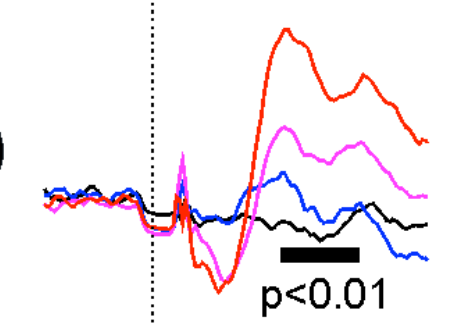

AMi

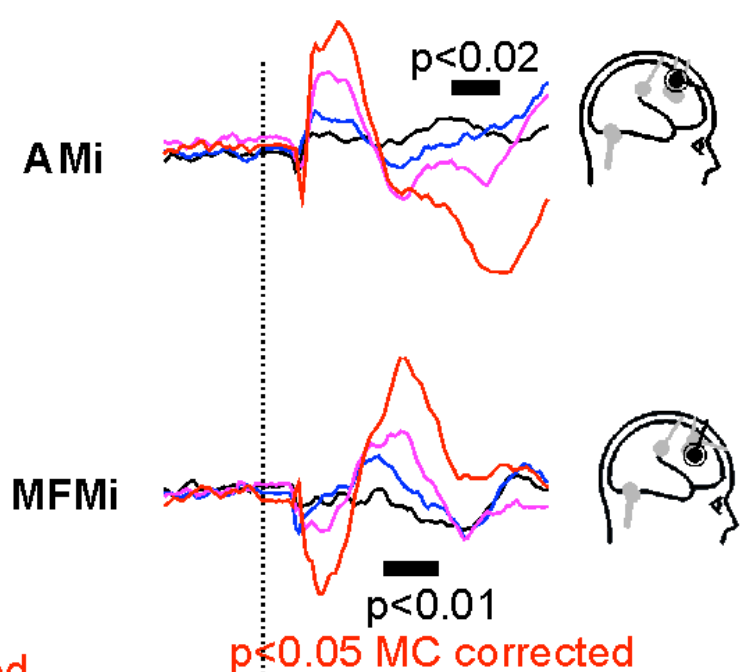

MFMi

${ }^{2}+4$

p $\leqslant 0.02 \mathrm{MC}$ corrected

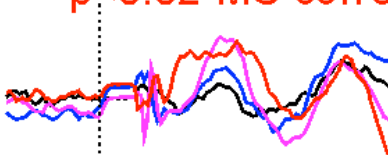

$\sqrt{9}, 7$

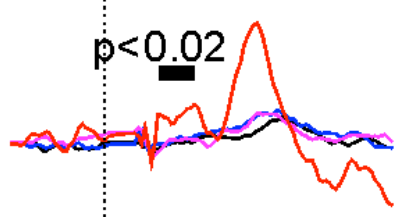

MFMc
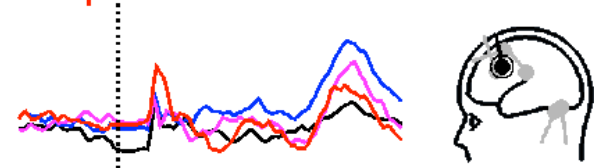

Cbi
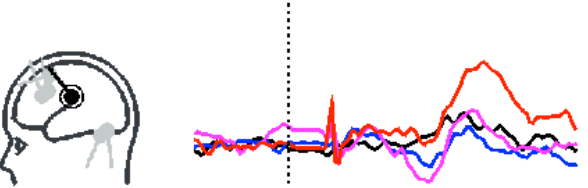

TPJi
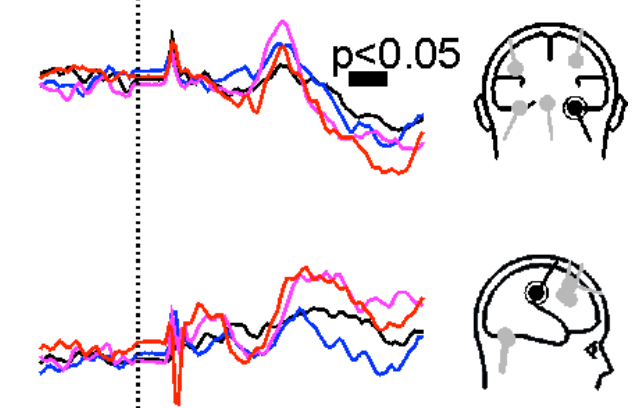

$\mathrm{CBm}$
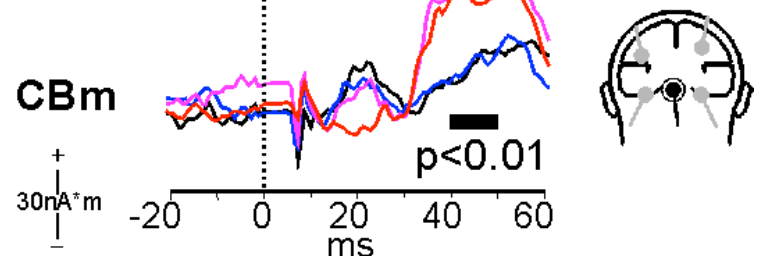

Figure 19. EEG responses dependence on stimulus intensity of $M 1$ stimulation. Grand average source waveforms for the four intensities are shown $(60 \%$-black, $80 \%$-blue, $100 \%$-magenta, $120 \%$-red). The black bars indicate time periods where significant intensity dependence was found for a particular source. The p-values in black are uncorrected for multiple comparisons. 


\subsubsection{Discussion}

\subsubsection{The physiological significance of the findings}

\subsection{Temporal sequence of the response}

Exploring the brain's functional connectivity has always been an important motivation behind the attempts to combine magnetic stimulation with EEG recording (Komssi and Kahkonen, 2006). The idea was to stimulate a particular cortical area and determine other areas to which the activity propagated at the early stages of the response. The first study using the sample-and-hold circuit of Virtanen et al. (Virtanen et al., 1999), using a minimum norm inverse solution, showed that following the stimulation of M1 the activity propagated in about $22 \mathrm{~ms}$ to the homologous area in the other hemisphere (Ilmoniemi et al., 1997). However, since that first promising study subsequent attempts to present a sequence of activation of cortical areas have been scarce (Komssi et al., 2002; Massimini et al., 2005a). Our analysis suggests a detailed sequence of events following left and right M1 stimulation that partially disagrees with but also extends the previous findings.

As could be expected, the earliest response was observed close to the stimulation site. The early negativity seen at $11 \mathrm{~ms}$ for left M1 and at $10 \mathrm{~ms}$ for right M1 stimulation (Figure 17) is probably the immediate response of the tissue directly stimulated by TMS. This response was obstructed by the disconnection of the recording system and the residual artifact and therefore was not modeled in the present study. In the next stage activation was seen in the tangentially oriented AMi source around $17 \mathrm{~ms}$ for the left M1 and 13ms for the right M1. The coordinates of both left and right AM sources are consistent with a location close to the middle frontal gyrus, at Brodmann area 6 . The tangential orientation of the ECD indicates that the cortical generator is located in a wall of a sulcus. Based on the studies of a variety of sensory cortical responses using current source-density Mitzdorf (Mitzdorf, 1985) suggested that the initial response of a sensory area to thalamic input recorded above the cortical surface consists of a positivity produced by current flow from the cell bodies to apical dendrites of pyramidal cells in layer 4 , followed by a negativity produced by current flow in the opposite direction as a result of synaptic input at the apical dendrites. If 
this basic sequence also applies to our case then the generator corresponding to the AM source should be located at the posterior wall of a sulcus so that the cortical surface there will be oriented in the anterior direction. In this case the posterior wall of the precentral sulcus would be a plausible location. However, this should be taken with caution because it is likely that this generator is activated via cortico-cortical connections rather than thalamus and it is not clear what the expected response polarity in this case would be.

The next source to respond was MFMi. Its response consisted of a negative peak approximately at the same latency as the AMi response and a positive peak between 20 and $30 \mathrm{~ms}$, which is clearly seen in the raw data. The ECDs fitted to this response seem to be relatively deep below the cortical surface and their location is consistent with a source at the cingulate gyrus. However, in this case the response sequence would be opposite to that suggested by Mitzdorf (Mitzdorf, 1985). An interesting possibility that might explain this discrepancy would be if the generator is located in the superior wall of the cingulate sulcus where the cortical surface is oriented downwards. Thus, a response sequence of positivity followed by negativity above the surface is seen with opposite polarity on the scalp. Physiologically this hypothesis places the generator in the supplementary motor area (SMA). Based on a similar logic, the generator can be placed even deeper - in the superior wall of the callosal sulcus, which would place it physiologically in the cingulate motor area (CMA).

The responses in the cerebellum modeled by the sources Cbi and Cbm have not been previously described in EEG studies. On the one hand, existence of these responses should not be surprising since the motor cortex projects to neo-cerebellum via the pontine nuclei. On the other hand, the responses observed in the present study were mainly accounted for by the ipsilateral and the median cerebellar sources, whereas M1 projects to the contralateral cerebellum. Furthermore, the contralateral cerebellum was found in previous studies to participate in the motor planning network (Schnitzler et al., 2006). Further studies are necessary to confirm the existence of these cerebellar responses and to further characterize them.

The responses in the TPJ sources might be either direct consequence of the magnetic stimulation coming perhaps from the somatosensory system or physiological artifacts 
such as middle latency auditory responses to the coil click or somatosensory responses to scalp stimulation.

The model revealed remarkable symmetry between the responses to stimulation at the two sites. Although there are both structural and functional differences between the two hemispheres (Haaland and Harrington, 1996), the basic sequence of events following M1 stimulation seems to be the same in both cases. The latencies of the responses in the right hemisphere seem to be several milliseconds shorter than the latencies of the analogous responses in the left hemisphere.

The response latencies revealed by our model can be compared with propagation delays obtained in paired-pulse stimulation studies in monkeys (Cerri et al., 2003; Shimazu et al., 2004) and in humans (Civardi et al., 2001; Daskalakis et al., 2004; Baumer et al., 2006; Koch et al., 2006). In these studies a conditioning stimulus was applied before the test stimulus at M1 to another motor cortical area at varying time intervals. This kind of study provides information about the range if inter-stimulus intervals for which the EMG response to the test stimulus is affected by the conditioning stimulus. These roughly correspond to the propagation delays between the conditioning site and M1. In the study of Civardi et al. (Civardi et al., 2001) conditioning TMS stimuli were applied 3-5 $\mathrm{cm}$ anterior to the hand motor area (that might correspond to our AMi source) or $6 \mathrm{~cm}$ anterior to the vertex on the nasioninion line (that might correspond to our MFMi and MFMc sources). In both cases the effect on M1 was inhibitory and the optimal delay was about $6 \mathrm{~ms}$. Similar delays were found between left and right M1 (Baumer et al., 2006). In the study of Daskalakis et al. (Daskalakis et al., 2004) conditioning at the cerebellum affected M1 with a delay of about 5-7 ms. These values are consistent with the fact that corticocortical connections conduct at a velocity of about 10-20 m/sec (Kandel et al., 2000). Therefore, theoretically, excitation can propagate from M1 to any other cortical area in the first $10 \mathrm{~ms}$ and we would expect that the earliest EEG responses to TMS are completely missed by our recording method due to the blocking of recording immediately after the stimulus. There is no way to refute this suggestion in the framework of the present study. However, in practice, it is not likely to be the case since delays between ERP peaks are comprised not only of the time of propagation in axons, but also of the processing delays at the cellular and network level. Thus, it may take some time between the arrival of the first action potentials from another brain 
area and the time that a potential gradient detectable on the scalp is generated by the pyramidal cells. An interesting analogy for this might be found the study of Williams and Baker (Williams and Baker, 2005) who showed, in a model of corticomuscular conductance, how a phase difference of $34 \mathrm{~ms}$ between neural oscillation in the cortex and in the muscles can arise with corticomuscular conduction time set to only $20 \mathrm{~ms}$. Factors such as synaptic potential duration and action-potential generation details were found to contribute to the observed delay. Thus, it would be surprising to find delays between activations of ECD model sources which are shorter than expected conduction times between the corresponding brain regions, but it would not be surprising to find delays which are much longer. In this sense our results are consistent with paired pulse stimulation data. Some constraints can also be drawn on the possible propagation pathways from the known conduction delays. For instance, since in the case of left M1 stimulation MFMi and Cbi were activated with a very short delay of about $4 \mathrm{~ms}$, it is unlikely that the input to cerebellum comes from the SMA or CMA and more likely that it comes from M1.

\subsection{Intensity dependence of the EEG responses to TMS}

Significant intensity dependence was found in several aspects of the response. The strongest and most significant dependence was found in the positive phase of the MFMi response and also at the late phase of AMi and the cerebellar responses. For the other response peaks one should differentiate between the cases where there was no intensity dependence at all and cases where intensity dependence appeared to exist in the grand average (Figure 19), but it cannot be shown statistically due to variability in the shape of the response across subjects. The latter is true for the early peaks of the AMi and MFMi sources. This might have to do with residual artifact at this time period that averaged out in the grand average but still affected the statistical analysis.

There seem to be several modes of intensity dependence. In some cases the response increased linearly with the stimulation strength (positive peak of the MFMi, positive peak of $\mathrm{AMi}$ in the right M1 stimulation), whereas in other cases there was a threshold phenomenon - a response only to high intensity stimulation. In some cases the threshold was between 100\% and 120\% RMT (AMi left, CBi left, Cbm left) and in other cases between $80 \%$ and 100\% RMT (TPJi right, Cbm right). Although the 
limitations of the present study (see below) do not allow detailed statistical analysis of these phenomena, the ECD modeling of the response combined with statistical analysis of the source waveforms are a promising method for performing such detailed analysis in the future.

The use of significance threshold uncorrected for multiple comparisons (black bars in Figure 19) was expected to result in some false positives and the cluster in Cbi between 12-20 ms seen for left M1 stimulation is likely to be such a case since its latency is too short compared to other cerebellar responses.

\subsection{Relation with the results of other TMS-EEG studies}

Several studies published in recent years examined the multichannel-EEG responses to TMS at M1 (Ilmoniemi et al., 1997; Paus et al., 2001a; Komssi et al., 2002; Komssi et al., 2004; Bonato et al., 2006). The results of these studies have been remarkably consistent and the present study improves understanding of this previously revealed pattern. The studies differ with respect to the earliest time that was examined but roughly all report a dipolar pattern ipsilateral to stimulation with an anterior negativity and a posterior positivity between 10 and $20 \mathrm{~ms}$ after the stimulus (sometimes referred to as P15), the broad vertex-positive pattern around $30 \mathrm{~ms}$ after the stimulus (P30) and a dipolar pattern ipsilateral to stimulation with opposite polarity to that of P15 around 45ms after the stimulus (N45). In our model P15 and N45 are accounted for by the AMi source and P30 by the MFMi source with a small contribution of MFMc.

Paus et al. (Paus et al., 2001a) discussed the possible origins of P30 and N45 and their conclusion was that only N45 originates in the motor cortex - consistent with our model. In a recent study from the same group (Van Der Werf and Paus, 2006) N45 amplitude was shown to be affected by $1 \mathrm{~Hz}$ rTMS delivered at M1 but not at dorsal premotor cortex, which again points at M1 as a likely generator. Another finding of Paus et al. (Paus et al., 2001a) was that P30 amplitude does not correlate with TMS intensity. This contradicts our finding of a highly significant correlation of the MFMi response with stimulus strength (Figure 19). Perhaps the discrepancy originates in a difference in the set of amplitudes tested. 
Another important contradiction with some of the previous studies (Ilmoniemi et al., 1997; Komssi et al., 2002) is the absence of a strong response in the contralateral M1. Response was observed in the MFMc source primarily to left M1 stimulation. However, the fact that it coincided in time with the MFMi response and both sources had overlapping scalp projections does not rule out the possibility of crosstalk. In any case the contralateral responses found in the present study were weak and close to the midline and we did not observe the contralateral dipolar pattern at about $24 \mathrm{~ms}$ poststimulus, as reported in Ilmoniemi et al. (Ilmoniemi et al., 1997). Such a pattern was also absent in several other studies (Paus et al., 2001a)\}(Komssi et al., 2004; Bonato et al., 2006). Thus, the question whether responses in contralateral M1 can indeed be recorded at least in some subjects requires further clarification.

\subsection{Relation with the results of studies using other imaging methods}

Several previous studies examined the effect of TMS stimulation at M1 using imaging methods other than EEG. In a recent paper Denslow et al. (Denslow et al., 2005) suggest a scheme of all the brain areas that are expected to be affected by TMS at M1, based on our knowledge about the motor system and physiological side effects of magnetic stimulation (Figure 20). For any of the areas listed in this figure fMRI or PET studies can be found showing activation. Yet, overall there is great disparity between the results which might be related to differences in stimulation protocols and the imaging methods used. The common elements seen in all the studies are activation at the stimulation site and auditory activation due to the coil click. In the present study this would correspond to the AMi source and possibly the TPJ sources respectively. A question of special relevance to our results is whether there is activation that could correspond to our MFM sources i.e. relatively deep activation close to the midline. This is indeed found in the fMRI study of Li et al. (Li et al., 2004) where activation at the cingulate gyrus (Brodmann area 32) was found in response to single TMS pulses delivered at the left M1, in the study of Bestmann et al. (Bestmann et al., 2004) where activation was found at both SMA and CMA in response to $3.125 \mathrm{~Hz}$ repetitive TMS and in the study of Baudewig et al. (Baudewig et al., 2001) where SMA was activated by $10 \mathrm{~Hz}$ rTMS. Interestingly, the response in both areas was found to increase with TMS intensity (Bestmann et al., 2004) which would be consistent with the properties of the MFMi source in our study. 


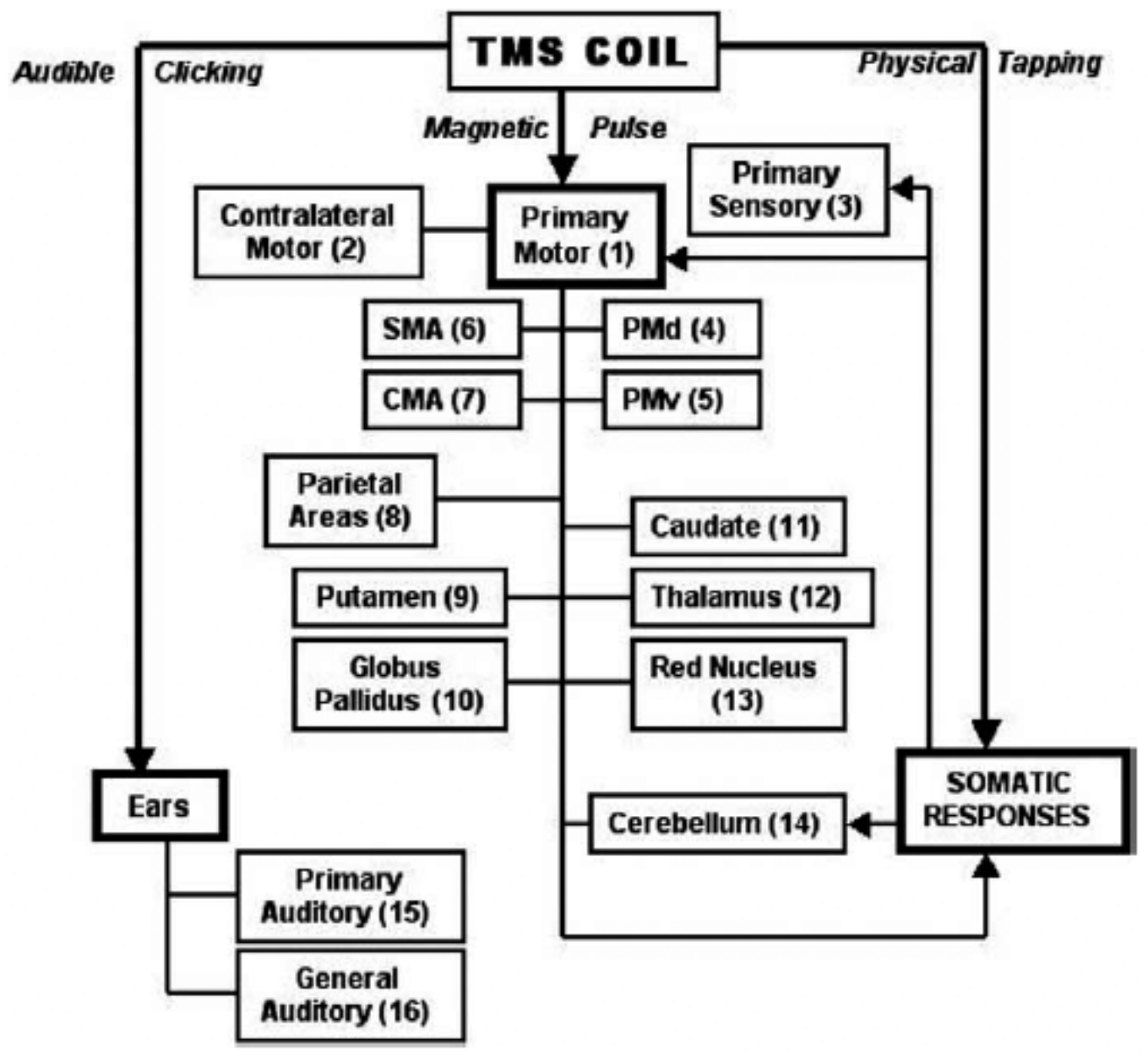

Figure 20. Overview schematic of areas that may be functionally connected to the primary motor area of the cortex. From (Denslow et al., 2005).

Finally, activation in the cerebellum was also found in the study of Bestmann et al. (Bestmann et al., 2004) and Baudewig et al. (Baudewig et al., 2001). In the latter study cerebellar activation ipsilateral to stimulation was described.

For many of the areas found in fMRI studies to be activated there are no clear correlates in the EEG. This is not surprising given the difference in the experimental protocols and also in the types of brain activity that can be detected by the different imaging methods. Nunez and Silberstein (Nunez and Silberstein, 2000) discuss the latter issue in detail and conclude that it is quite likely that activity detectable by EEG will not be detectable by fMRI and vice versa. Nevertheless, some utility can be drawn from the comparison between the two kinds of results. 


\subsubsection{Limitations of the present study}

\subsection{The amount of data}

The present study is based on previously acquired data and therefore the analysis had to be adjusted to accommodate the limitations of the existing dataset. The original studies of Komssi et al. (Komssi et al., 2004) and Kahkonen et al. (Kahkonen et al., 2005b) were aimed at comparing responses to stimulation at different sites and different intensities. Thus, in order to keep the experiment manageable and stay within the safety bounds with respect to the number of stimuli delivered to each subject (Wassermann, 1998) the number of stimuli for each condition had to be limited to 100. Following the artifact rejection in the present study the average number of trials analyzed varied between 85 and 90 depending on the condition. While these numbers are sufficient to analyze the grand average responses and perform statistical analysis across subjects, they are insufficient to characterize the responses of each of the subjects and systematically analyze the similarities and differences between subjects. Such analysis would definitely be of interest for constructing individual functional connectivity maps and using the TMS-EEG combination for diagnostic purposes. For the same reason we did not perform analysis of peak amplitudes and latencies. Since the shapes of the responses greatly varied between subjects, the definition of individual peaks would be subjective and imprecise. These issues can be addressed in future studies with smaller number of experimental conditions.

\subsection{The residual artifact}

Our examination of the data recorded with the sample-and-hold circuit (Virtanen et al., 1999) showed that the circuit does not block the TMS artifact completely, especially with high stimulation intensities. In the original publications (Komssi et al., 2004; Kahkonen et al., 2005b) the authors dealt with this problem by excluding channels contaminated by the residual artifact from the analysis. This method may be biased toward eliminating electrodes close to the stimulation site and can therefore lead to loss of important information about the early stages of the response to TMS. In addition, the number of excluded channels is expected to increase with higher stimulation strength and this would introduce an additional bias when comparing 
different intensities. Exclusion of channels from a particular part of the scalp is likely to introduce biases in source localization (Michel et al., 2004).

We show that the topography based method originally proposed for correction of eye movement artifacts is well suited for correction of TMS induced artifacts. The combination of hardware sample-and-hold circuit and a software artifact correction algorithm makes it possible to study the early stages of the response that would be the most relevant for study of functional cortical connectivity.

\subsection{Advantages and disadvantages of our modeling approach}

In the present study we used an equivalent current dipole model using a sequential fitting strategy. This approach is only one of a variety of methods for solving the so called 'inverse problem' i.e. computing the current source density in the cortex from EEG data recorded on the scalp (Hamalainen and Ilmoniemi, 1994; Scherg and Berg, 1996; Pascual-Marqui, 1999; Gross et al., 2001). Since the problem is underdetermined, the solution is not well defined by the data and additional assumptions are necessary. Some of the source localization methods compute the current source density from the scalp potentials according to a pre-defined formula based on method-specific assumptions. Such methods have the advantage of objectivity because they do not require that the experimenter manually constructs a source model. However, in the case of EEG responses to TMS they might produce erroneous results since they do not differentiate between the physiological cortical response and the TMS artifact. The process of manually constructing a multiple source model makes it possible to keep track of the relation between the scalp patterns in the raw data and the model sources accounting for these patterns and to avoid modeling patterns that are clearly artifacts. In the particular case of the present study, the symmetry of responses to the left and right M1 stimulation that clearly emerged from the raw data (Figure 17) could be used as a constraint in the model construction. Taking this symmetry into account as a constraint for a solution such as minimum norm (Hamalainen and Ilmoniemi, 1994) or LORETA (Pascual Marqui et al., 1994) is a highly non-trivial task and would not have general applicability.

There are several possible lines of criticism of the multiple ECD approach. A point dipole is not a perfect model for actual cortical sources which are areas of at least 
several centimeters squared. However, modeling studies have shown that the electrical field generated by an array of identically oriented sources covering an area of up to $3 \mathrm{~cm}^{2}$ can be well modeled by a single dipole (Scherg and Hoechstetter, 2000). Therefore the ECD approach does not assume that the cortical sources are point dipoles but only approximates them as such. Doing so makes it possible to represent all activity with similar temporal and spatial features by a single source whose position and activity waveform can then be further analyzed. Conversely, current-source density estimation on a grid leaves open the problem of defining the sources. Thus, in order to analyze the time-course of activity, a region of interest has to be defined; a definition which is subjective and might lead to confounding unrelated activities. Another important assumption of the ECD approach is that there are discrete regions of activation which are activated sequentially rather than continuous propagation of a wave of excitation over the cortical surface. While multiple studies (Scherg and Von Cramon, 1986; Scherg and von Cramon, 1986; Bast et al., 2005) have shown that the former description is appropriate for the ERPs evoked by sensory stimulation, the latter mode of activity propagation can possibly be found only in some cases of epileptic seizures. The studies of TMS evoked activity performed to date support the idea that although the initial TMS activation of the cortex is non-physiological, the subsequent propagation of activity resembles the normal rather than pathological processes in the cortex. In the visual system focal magnetic stimulation produces visual sensations limited to small region of the visual field and in the motor system activation of a well defined set of muscles. There has been no evidence of continuous wave-like propagation of activity in normal subjects. Moreover, the sequence of the raw ERP scalp maps in the present study (Figure 17) is consistent with several discrete sources with characteristic scalp signatures rather than a continuous drift of a single source.

An additional subjective decision that should be made while building a multiple ECD model is about the number of sources. While this parameter is not unambiguously determined by the data, the decision can be based on examining the residual activity not accounted for by the initial model with only one source or two symmetric sources. Additional sources are added as long as the residual contains clear peaks. The advantage of this process is the ability to roughly estimate the number of distinct generators involved in the response. Such information cannot be obtained from current source density estimation as discussed above. Current density estimation 
procedures (e.g., LORETA) make no assumptions on the number of generators, but the solution does not include the source orientation.

\subsubsection{Conclusions}

The methodology developed in the present study made it possible to substantially reduce the TMS stimulation artifact even for high stimulation intensities. This paves the way to applying TMS-EEG imaging to a variety of stimulation sites and conditions many of which were not accessible until now. Based on artifact-corrected data we propose for the first time a multiple source model for the early phase of the response evoked by magnetic stimulation of left and right M1. A remarkable symmetry was found between the two cases. The main sources contributing to the response according to our model are the stimulated motor cortex and a deep ipsilateral source close to the midline, possibly SMA or CMA. We also discovered responses in the cerebellum which have not been described previously. The results of the study were consistent with previous EEG and fMRI studies. 


\subsection{LTP-like changes induced by paired associative stimulation of the primary somatosensory cortex in humans: source analysis and behavioral correlates}

\subsubsection{Introduction}

Long-term potentiation (LTP) and long-term depression (LTD) refer to long-lasting modifications of synaptic transmission and are believed to represent important physiological substrates of learning and memory (Bliss and Gardner-Medwin, 1973; Bliss and Lomo, 1973; Martin et al., 2000; Malenka and Bear, 2004; Cooke and Bliss, 2006). Animal experiments have shown that synaptic efficacy can be modified based on the principles of associativity and input specificity. In some circumstances, whether LTP or LTD is induced, depends on the temporal order of pre-and postsynaptic events (Levy and Steward, 1983; Mulkey and Malenka, 1992; Kirkwood and Bear, 1994; Froemke et al., 2005; Dan and Poo, 2006). Compelling evidence that LTP may underlie some forms of learning and memory has been obtained from studies of hippocampal and amygdalar synaptic plasticity (Morris et al., 1986; Barnes et al., 1994; Martin et al., 2000). Such correlation between LTP/LTD formation and behavior has also been obtained in neocortical sensory systems. For instance, in the visual system of cats, precisely timed visual stimuli induced specific receptive field plasticity depending on the temporal sequence of neuronal events, and similar stimuli changed the performance in visual discrimination tasks in humans (Yao and Dan, 2001; Fu et al., 2002).

Given the central importance of LTP for memory formation and learning, several groups (Stefan et al., 2000; Mao and Evinger, 2001; Yao and Dan, 2001; Fu et al., 2002; Schorr and Ellrich, 2002; Dinse et al., 2003; Klein et al., 2004; Huang et al., 2005; Teyler et al., 2005) have attempted to generate models of LTP/LTD in humans by using non-invasive stimulation protocols. The evidence linking the neuronal excitability changes induced by these protocols to LTP/LTD was based on the similarity of the induction protocols to those used in animals, and on the physiological and pharmacological properties of induced excitability changes. However, little is known about the regional and laminar location and behavioral significance of these LTP-like changes. One of the protocols, developed by our group, was shaped after animal models of associative LTP/LTD (Stefan et al., 2000). This protocol, termed paired associative stimulation (PAS), uses repetitive magnetic cortical stimulation 
timed to interact with median nerve $(\mathrm{MN})$ stimulation-induced afferent signals from homotopic body regions. The PAS-paradigm was shown to induce long-lasting (>30 $\mathrm{min})$, yet reversible changes of median nerve somatosensory evoked potentials (MNSSEP) (Wolters et al., 2005) evident in single-channel recordings from the scalp region overlying the somatosensory cortex. These changes depended on nearsynchronicity of induced events and their direction changed as a function of the exact interval between the two stimulation modalities within a short time window of just 15 ms. Magnetic stimulation delivered at $-5 \mathrm{~ms},-2.5 \mathrm{~ms}$ and $0 \mathrm{~ms}$ relative to the subject's MN-SSEP N20 peak was shown to induce a long-lasting increase in the amplitude of MN-SSEP P25 peak, whereas magnetic stimulation delivered $20 \mathrm{~ms}$ before the N20 induced a long-lasting decrease in the amplitude of P25. PAS-induced changes were confined to the body region receiving dual input from afferent stimulation and magnetic cortex stimulation.

However, with single-channel recordings, inferences about the location of induced excitability changes within the brain are limited. Considerably stronger evidence relating PAS-induced excitability changes to certain regions within the primary somatosensory cortex would be provided by using multichannel recordings (Buchner et al., 1995; Restuccia et al., 2002). In the present study we used a combination of established and novel, data-driven statistical approaches (Nichols and Holmes, 2002; Maris, 2004; Helen Brown and Prescott, 2006; Maris and Oostenveld, 2006) to determine the presence and topographical distribution of PAS-induced excitability changes as a function of the timing and composition of somatosensory afferent stimulation induced by MN-stimulation with respect to the TMS-pulse. We hypothesized that topographically differential effects on MN-SSEP changes would be induced by near-synchronous interaction of TMS-evoked events with afferent signals differing in the composition of their somatosensory quality and that MN-SSEP changes should be absent with asynchronous application of the two stimulation modalities. In addition, the question of behavioral correlates of PAS-induced excitability changes was addressed. We hypothesized that PAS-induced MN-SSEP changes should be correlated with behavioral effects conditional upon qualitative congruency of Hebbian stimulation. Finally, based on previous findings (Wolters et al., 2005) we investigated the question if the direction of behavioral changes depended on the interval between MN-stimulation and TMS-pulse. 


\subsubsection{Methods}

\subsubsection{Subjects}

The protocol conformed to the principles of the declaration of Helsinki and was approved by the Ethics committee of the University of Würzburg. Experiments were performed on 11 healthy volunteers ( 8 men, 3 women), aged 20.9 to 44.5 (mean $26.3 \pm 7.2$ years) with normal results of neurological examination, who gave their written informed consent. All subjects were right-handed, by their own statement. Three additional subjects ( 1 male, 2 female) participated in the project at the initial stages but did not complete the series of five experiments and were excluded from further analysis.

\subsubsection{MRI scan}

Anatomical magnetic resonance imaging (MRI) scans were obtained from all the subjects for subsequent use for TMS navigation and EEG source imaging. Magnetic resonance tomography was performed using a Magnetom Vision 1.5-Tesla-Scanner (Siemens, Erlangen, Germany) utilizing a circular standard head coil following a routine imaging protocol. A conventional axial T1-weighted sequence was performed using a slice thickness of $1 \mathrm{~mm}$.

\subsubsection{Stimulation}

Electrical nerve stimulation was performed with an electrical stimulator (Digitimer D7AH, Digitimer, Welwyn Garden, Herthfordshire, UK) with a standard stimulation block (cathode proximal). Transcranial magnetic stimulation (TMS) was performed using a flat figure-eight shaped magnetic coil (diameter of each wing: $70 \mathrm{~mm}$ ) connected to a Magstim 200 magnetic stimulator (Magstim, Whitland, Dyfed, UK). The coil was held tangentially to the skull with the handle pointing backward and laterally at an angle of $45 \mathrm{deg}$ to the sagittal plane.

\subsubsection{Recording}

Electroencephalographic (EEG) signals were recorded from the scalp with a 64channel Quickamp system (Brain Products GmbH, Munich, Germany). The electrodes 
were located at extended 10-20 system locations and fixed on the subject's head with an elastic cap (EASYCAP GmbH, Herrsching-Breitbrunn, Germany). The electrode impedance was kept below $5 \mathrm{k} \Omega$ and verified before each recording. Electrode positions and anatomic reference points were measured for each subject with a $3 \mathrm{D}$ navigation system (Brainsight, Rogue research, Montreal, Canada). The brain signals were average referenced in hardware, filtered between 0 and $560 \mathrm{~Hz}$ and sampled at $2000 \mathrm{~Hz}$. Surface electromyographic (EMG) activity was recorded from the right abductor pollicis brevis muscle (APB) using $\mathrm{Ag}-\mathrm{AgCl}$ electrodes (Fischer Medizintechnik, Nürnberg, Germany) with the active electrode mounted on the muscle belly and the reference electrode placed over the base of the metacarpophalangeal joint of the thumb. To exclude the possibility that changes of MN-SSEP amplitudes were related to variable efficiency of peripheral nerve stimulation following the PAS intervention, median nerve sensory nerve action potentials (SNAPs) were recorded antidromically from digit 2 using ring electrodes. EMG and SNAP signals were amplified (CED1902 amplifier, Cambridge Electronics Design, Cambridge, UK) and bandpass filtered between $5 \mathrm{~Hz}$ and $2000 \mathrm{~Hz}$. EMG and SNAP data were sampled at $5000 \mathrm{~Hz}$, digitized using an A/D converter (CED1401 plus, Cambridge Electronics Design, Cambridge, UK) and stored in a laboratory computer for display and later off-line analysis.

\subsubsection{Experimental procedures}

The principal purpose of all experiments was to determine the effect of a paired associative stimulation protocol on MN-SSEPs recorded with multichannel EEG and on tactile performance as assessed by two-point discrimination. Subjects were seated in a comfortable chair.

\subsection{Search for somatosensory hot spots and threshold measurement}

The optimal position of the magnetic coil over the left motor cortex for eliciting motor evoked potentials (MEP) in the right APB, termed "motor hot spot", was determined at moderately suprathreshold stimulation intensity. The coil position during the search for the motor hot spot was continuously monitored with a $3 \mathrm{D}$ navigation system registered to the subject's MRI scan. When the motor hot spot was found, the optimal position and orientation of the TMS coil for eliciting MEPs at this spot was marked 
directly on the scalp with a soft-tip pen and digitally recorded with the Brainsight system. Using the scalp markings and a cardboard replica of the coil, the actual position of the motor hot spot was determined. The hand representation at primary somatosensory cortex ("somatosensory hot spot"), was then marked at $2 \mathrm{~cm}$ posterior to the motor hot spot. The TMS coil was then positioned over S1 and oriented in parallel to the optimal orientation at the motor hot spot. Position and orientation were recorded with the Brainsight system. Off-line analysis of the trajectory of the coil projection onto the individual brain anatomy revealed that the somatosensory hot spot corresponded to the location of the inverted Omega shape that characterizes the location of the primary somatosensory cortex in humans (Okamoto et al., 2004). This is consistent with previous results from other groups (Herwig et al., 2003).

Following application of the EEG cap and reduction of the impedance of the electrodes to below $5 \mathrm{k} \Omega$, the resting motor threshold (RMT) at the motor hot spot was measured. RMT was defined as the stimulus intensity needed to produce a response of at least $50 \mu \mathrm{V}$ in the relaxed APB in $50 \%$ of the trials at resolution of $1 \%$ of the maximal stimulator output. The RMT value was determined using the Maximum Likelihood Threshold Hunting (MLTH) procedure (Awiszus, 2003; Mishory et al., 2004). In this procedure TMS pulses are delivered at intensities computed by the MLTH routine so that the outcome of the stimulation would be the most informative for threshold determination at each stage. The outcome of each trial (success or failure to evoke a suprathreshold MEP) is provided to the routine as input. After several trials the routine produces an initial RMT estimate which improves with each subsequent trial. We continued this procedure until it converged, i.e. the RMT estimate varied within less than $1 \%$ of the stimulator output in several consecutive trials. The RMT was $41 \pm 6 \%$ of the maximal stimulator output. On average $23 \pm 6$ TMS pulses were necessary to achieve convergence. Complete muscle relaxation was monitored by examining the MEP waveform at the baseline period and if necessary audio-feedback was used to help the subjects monitor their relaxation. Skull thickness is similar over M1 and S1 because the two areas are positioned similarly along the medio-lateral axes (Knecht et al., 2005). Therefore, the TMS stimulus intensity for the intervention was determined as a fixed multiple of the RMT. 


\subsection{Median nerve somatosensory evoked potentials}

Median nerve stimulation was performed using a pulse width $200 \mu$ s at a frequency of $3 \mathrm{~Hz}$ and a stimulation intensity of 300 percent of the individual perceptual threshold. The average perceptual threshold was $3.4 \pm 0.6 \mathrm{~mA}$. The stimulation intensity $(10.3 \pm 1.9 \mathrm{~mA})$ was in all cases above the minimal $\mathrm{MN}$ stimulation intensity necessary to evoke movements in the thumb $(7.5 \pm 2.2 \mathrm{~mA})$. Responses to 1800 stimuli were recorded before and after PAS intervention. EEG was recorded continuously and MNSSEPs were computed offline.

\subsection{Paired associative stimulation (PAS)}

The PAS-intervention followed a protocol published previously by our group (Wolters et al., 2005), which was a variant of the PAS protocol used for the motor cortex (Stefan et al., 2000; Wolters et al., 2003). In a typical experiment, repetitive single electrical stimuli were delivered to the right median nerve at the level of the wrist at 300 percent of the perceptual threshold, each followed by TMS delivered over S1. TMS was applied at an intensity of 1.5 times the resting motor threshold measured with the EEG cap on $\left(\mathrm{SI}_{1.5 * \mathrm{RMT}}\right)$. Taking all experiments into consideration, $\mathrm{SI}_{1.5 \mathrm{RMT}}$ amounted to $62 \pm 9 \%$ of the maximal stimulator output. Over 30 minutes, 180 pairs were delivered at $0.1 \mathrm{~Hz}$. Since it was previously shown that distraction can diminish the effect of PAS (Stefan et al., 2004), the subjects were instructed to attend to their right hand during the PAS intervention,. In order to control their attention a lightemitting diode (LED) was fixed to the right wrist in the proximity of the stimulating electrode. The experimenter turned the LED on between 5 and 13 times during the procedure. The subjects were instructed to count the number of light flashes and report the count at the end of the intervention. Subjects' counting accuracy was $90 \pm 15 \%$.

Following PAS, responses to additional $1800 \mathrm{MN}$ stimuli were recorded. The recording started after a short preparation $2.7 \pm 0.8 \mathrm{~min}$ after the end of PAS. Identical stimulation parameters were used before and after intervention. If necessary, the stimulating electrode was adjusted to produce sensory nerve action potentials of similar magnitude in both recordings thus ensuring similar MN stimulation intensity. 
The principal experimental design shared by all experiments is illustrated in Figure 21.

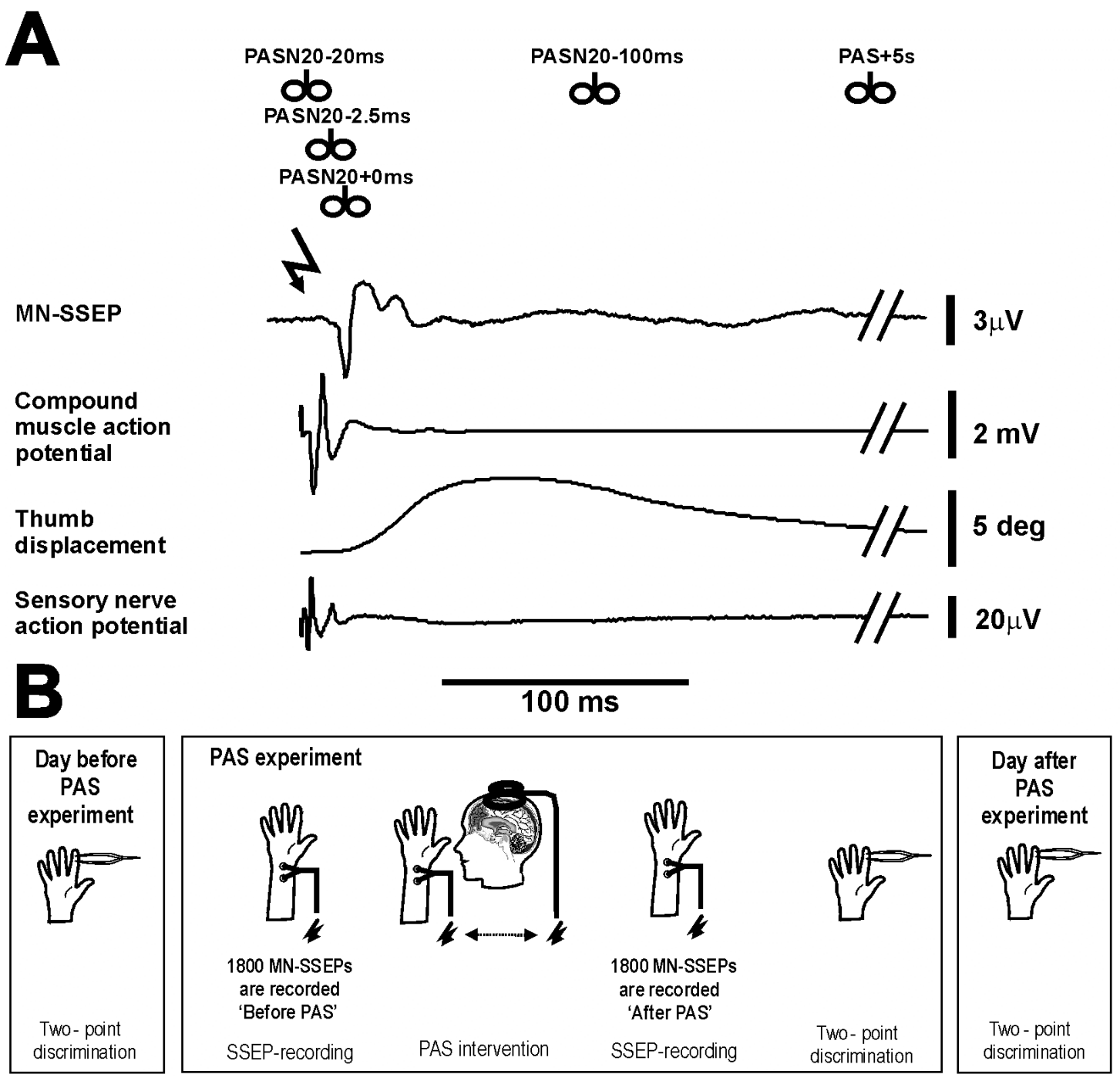

Figure 21. A. Semi-schematic illustration of the timing of various physiological events triggered by median nerve stimulation and of the different PAS-interventions used. Exemplary recordings from a single subject. From top to bottom: median-nerve somatosensory evoked potential (average of 1755 trials); positional thumb displacement as recorded by a fiber-optics based joint motion sensor, compound muscle action potential recorded from abductor pollicis brevis muscle, sensory nerve action potential recorded from digit 2 (average of 20 trials each). Arrow head indicates time of delivery of electric pulse to median nerve. Magnetic coil symbols indicate time of TMS pulse in relation to time of MN-stimulation and triggered physiological events 


\subsubsection{PAS intervals}

To manipulate the temporal sequence, the composition of the somatosensory quality, and degree of synchronicity of afferent signals with respect to TMS-evoked neuronal activity, TMS was delivered over S1 after the MN pulse at one of the following times relative to the subject's MN-SSEP N20-peak: $-20 \mathrm{~ms},-2.5 \mathrm{~ms}$, 0ms, $100 \mathrm{~ms}$ $(\mathrm{PASN} 20+\mathrm{X})$, and $\sim 5 \mathrm{~s}(\mathrm{PAS}+5 \mathrm{~s})$ (Figure 21A). The N20-latency was defined as the mean latency of the N20-component of the subject's MN-SSEPs recorded before PAS. The individual N20-latency was on average 20.0 $\pm 0.7 \mathrm{~ms}$. Electric compound MNstimulation at the level of the wrist orthodromically stimulates somatosensory afferents from mechanoreceptors as well as from muscle spindles and deep connective tissue sensors. Therefore, a TMS pulse delivered to S1 is near-synchronous to the arrival of this mixed-quality signal when delivered at an interval comparable to the individual N20-latency of the MN-SSEP, i.e. at $\sim 20 \mathrm{~ms}$ after the electric stimulus to the MN. As argued elsewhere (Wolters et al., 2005), varying the interstimulus interval from $\mathrm{ISI}=\mathrm{N} 20$-latency-20ms to $\mathrm{ISI}=\mathrm{N} 20$-latency-2.5ms or ISI=N20-latency $+0 \mathrm{~ms}$ changes the sequence between the late neuronal events induced by TMS and those events induced by afferent MN stimulation from a situation where postsynaptic events lead presynaptic events (PASN20-20ms) to one where pre-synaptic events lead late postsynaptic events (PASN20-2.5ms; PASN20+0ms). Additionally, MN-stimulation at 300 percent perceptual threshold leads to orthodromic activation of the $\mathrm{MN}$ motor axons with subsequent activation of APB muscle. The twitch of the APB muscle, in turn, leads to a second period of afferent activity traveling to S1. Of note, this activity contains mainly proprioceptive information and is, therefore, distinct from that set up by direct electric $\mathrm{MN}$ stimulation arriving at $\sim 20 \mathrm{~ms}$ at S1. Figure $21 \mathrm{~A}$ illustrates the timing of various physiological events triggered by $\mathrm{MN}$ stimulation in a single subject. After a delay caused by electromechanical transduction, the position of the thumb changes, with the positional displacement maximal at about $100 \mathrm{~ms}$ after the median nerve stimulation. Therefore, TMS directed to S1 at the individual N20latency $+100 \mathrm{~ms}$ will be near-synchronous to the proprioceptive afferent signals. As a control, the magnetic pulse was delivered at half time between two median nerve stimulations $(\mathrm{PAS}+5 \mathrm{~s})$.

To prevent subject and experimenter bias in behavioral examination, the PAS condition was set by another experimenter not involved in the two-point 
discrimination testing. At first PASN20-20ms and PASN20-2.5ms were performed. The order of these two conditions was balanced between subjects. The behavioral data for each subject was revealed only after the two experiments were completed. In the second stage of the study PASN20+0ms and PASN20+100ms were performed. Again, the order was balanced and the subjects and the experimenters performing the behavioral examination were blind to which of the two conditions was being tested. In the third stage of the project $\mathrm{PAS}+5 \mathrm{~s}$ was performed. For all but two subjects this was the last condition tested. Since a subject can identify this condition easily during PAS intervention due to the long interval between the paired stimuli, the behavioral examination could not be performed blindly and was, therefore, not performed for most of the subjects.

\subsubsection{EEG analysis}

The EEG data was processed using BESA EEG analysis software (MEGIS Software GmbH, Gräfelfing, Germany). The recordings made before and after PAS intervention were concatenated into a single file so the analysis was identical for both recordings. The continuous EEG data was high-pass filtered (cutoff frequency $20 \mathrm{~Hz}$, slope $6 \mathrm{db} /$ oct, zero phase) and epoched between -100 and $200 \mathrm{~ms}$ relative to the $\mathrm{MN}$ stimulus. Trials containing artifacts were rejected using the BESA artifact rejection interface (Scherg et al., 2004). Trials were considered artifact and excluded from averaging if they satisfied one of the following two conditions: (1) The amplitude difference between the largest and the smallest sample within the trial in any channel exceeded $102.2 \mu \mathrm{V}$. (2) The voltage difference between two adjacent samples in any channel exceeded $26.3 \mu \mathrm{V}$. In some cases, one or more (mean 1.4 \pm 1.9 , max 9) EEG channels were excluded from analysis in order to retain more trials. The reason for channels being excluded was without exception contamination by scalp muscle activity. The retained epochs were averaged to obtain MN-SSEP waveforms. To account for differences between the subjects in cap placement and head shapes and to enable statistical comparisons in cases when different subsets of channels were excluded, the data were interpolated from electrode locations recorded during the experiment to standard extended 10-20 locations of the corresponding channels using spline interpolation (Scherg et al., 2002). In order to identify cortical sources affected by PAS, we used the MN-SSEP source model of BESA. This model is based on the grand average of MN-SSEPs recorded from 20 subjects by Buchner (Buchner et al., 
1994) using a sequential fitting procedure similar to that described in (Buchner et al., 1995). We also performed fitting of an individual source model for each subject using the same procedure. The results of analysis based on these models were not qualitatively different and therefore are not presented here.

Statistical analysis of the interpolated MN-SSEP data and source activity waveforms was performed using the open source Fieldtrip toolbox for Matlab (http://www.ru.nl/fcdonders/fieldtrip/, F.C. Donders Centre for Cognitive Neuroimaging, Nijmegen, The Netherlands). The nonparametric statistical procedure implemented in this toolbox allows assessing the significance of spatio-temporal scalp patterns (Maris and Oostenveld, 2006; Takashima et al., 2006). This solves the problem of multiple comparisons that might otherwise lead to an inflation of type I error because t-test was performed at multiple electrodes and multiple time-frames. Using this procedure, it is possible to reveal significant effects occurring at any time within the epoch of interest and at any scalp location.

\subsubsection{Behavioral examination}

Simultaneous spatial two-point discrimination performance on the tips of the right index (D2) and little finger (D5) was tested in a two-alternative forced-choice tactile discrimination task (Pleger et al., 2001). Seven pairs of pins with separation distances of $0.7,1.0,1.3,1.6,1.9,2.2$, and $2.5 \mathrm{~mm}$, each pair mounted on a caliper, were used. In addition, zero distance was tested with a single pin. Stimuli were applied with moderate force (resulting in approximately $2 \mathrm{~mm}$ of skin indentation) by one of two different investigators who were blind to the protocol applied in the respective session. Each distance of the pins was tested 10 times in randomized order. The subject had to decide immediately whether he or she had the sensation of one or two tips. The subject's responses were assigned values of "0" (for "one tip") and "1" (for "two tips") and summed for each distance separately. These values were plotted against tip distance as a psychometric function. The threshold was read from the curve at the distance which corresponded to a level of $50 \%$ correct responses. When this level was above or below the range tested, the threshold was set to the highest $(2.5$ $\mathrm{mm})$ or the lowest $(0.7 \mathrm{~mm})$ tested value, respectively. 
In order to assess the net effect of PAS on the two-point discrimination threshold and neutralize other effects not relevant to the present study, such as differences in sensitivity between subjects, learning over the course of the project (along the 5 experiments) and systematic changes in sensitivity among the 3 testing sessions of the same experiment, we summarized the three thresholds that were computed for each digit in a single experiment in a single value $-\Delta 2 \mathrm{Pt}$ defined as follows:

$$
\Delta 2 P t_{S, I, D}=\Theta_{S, I, D}^{P A S}-\frac{\Theta_{S, I, D}^{\text {Before }}+\Theta_{S, I, D}^{\text {After }}}{2}
$$

where $\Theta_{S, I, D}^{B e f o r e}, \Theta_{S, I, D}^{P A S}$, and $\Theta_{S, I, D}^{A f t e r}$ are the two-point discrimination thresholds computed for subject $S$ in an experiment with PAS interval $I$ for digit $D$ at the day before the experiment, during the experiment following PAS and at the day after the experiment respectively. $\Delta 2 \mathrm{Pt}$ represents the deviation possibly induced by PAS from a linear trend between the values of the day before and day after PAS. This is shown schematically in Figure 26B. When $\Theta_{S, I, D}^{\text {Before }}, \Theta_{S, I, D}^{P A S}$, and $\Theta_{S, I, D}^{A f t e r}$ fall on the same line $\Delta 2 P t_{S, I, D}$ is equal to zero. Negative $\Delta 2 \mathrm{Pt}$ means that the actual threshold was lower than expected, indicating improvement in discrimination ability whereas positive $\Delta 2 \mathrm{Pt}$ indicates performance worse than expected. We excluded from the analysis the measurements in which a subject failed to achieve at least $60 \%$ accuracy in identifying the single pin. In addition, based on the Kolmogorov-Smirnov test of the normal distribution assumption, a single outlier was excluded from the behavioural analysis for PASN20-20ms condition. In these cases we could not compute $\Delta_{S, I, D}$ and this resulted in missing values. A mixed model (Helen Brown and Prescott, 2006) was used to evaluate the statistical significance of the PAS effect. This model takes into account the repeated measures structure of the data. The procedure PROC MIXED of the SAS software (SAS Institute Inc., Cary, NC, USA) that we used to fit this model has the advantage of being able to fit this model for data with missing values.

The following model was fitted to the data:

$$
\Delta 2 P t_{S, I_{k}}=\mu+\alpha_{S}+\sum_{k=1}^{3} \beta_{k} W_{k}
$$


where $\mu$ is a fixed intercept, $\alpha_{S}$ is a subject-specific random effect (that accounts for the fact that the data coming from repeated measurements from the same subjects), $W_{k}$ is an indicator variable for PAS condition with interval $I_{k}$.

The $\Delta 2 \mathrm{Pt}$ parameter summarizes in a single value the change in the two-point discrimination observed at a single PAS experiment for one of the digits tested. The nonparametric statistical analysis framework (Maris and Oostenveld, 2006) makes it possible to test for correlation between an external parameter and evoked activity on the scalp. We tested for correlation between $\Delta 2 \mathrm{Pt}$ and the scalp map difference between the "After PAS" and "Before PAS" condition across subjects separately for each PAS condition and separately for D2 and D5. As in the case of nonparametric ttest, the nonparametric regression procedure identifies spatio-temporal clusters significantly correlated with $\Delta 2 \mathrm{Pt}$, while addressing the problem of multiple comparisons. 


\subsubsection{Results}

\subsubsection{PASN20-2.5ms and PASN20+100ms significantly affect MN-SSEP amplitude between 20 and 31 ms post-stimulus}

Figure 22A shows MN-SSEP waveforms in CP3-FCz montage comparable with previous MN-SSEP recordings of Wolters et al. (Wolters et al., 2005). Analysis of P25 peak amplitude showed a significant increase for PASN20-2.5ms and no significant effect for other conditions (Figure 22B). Figure 23 summarizes the analysis of MN-SSEP scalp map differences between the "After PAS" and "Before PAS" recordings. Significance was assessed with a permutation-based nonparametric statistical test applied at a time window between 15 and $35 \mathrm{~ms}$ after the stimulus. Since five PAS intervals were tested, the significance threshold was set to $p=0.01$ to correct for multiple comparisons over different PAS conditions. Significant differences were found for PASN20-2.5ms between 21 and $31 \mathrm{~ms}$ and for PASN20+100ms between 20 and $29 \mathrm{~ms}$ after the median nerve stimulus $(\mathrm{p}<0.01)$. In the case of PASN20-2.5ms a significant positive difference was found in the posterior left (contralateral) part of the scalp and a significant negative difference was found at the fronto-central part at the same time. In the case of PASN20+100ms a significant positive difference was found at a more posterior and medial part of the scalp relative to PASN20-2.5ms. There was also a negative difference at the frontal part of the scalp for PASN20+100ms, but it did not reach significance after Bonferroni correction ( $p=0.045$ for 5 comparisons). Similar results were obtained when all data were realigned to the timing of the individual N20-peak (data not shown). By contrast to the significant changes of MN-SSEP amplitudes following PASN20-2.5ms and PASN20+100ms interventions, sensory nerve action potential amplitudes remained essentially constant (mean percent increase $2.9 \%$ and $3.3 \%$, respectively). No significant differences were detected for the other PAS conditions. 

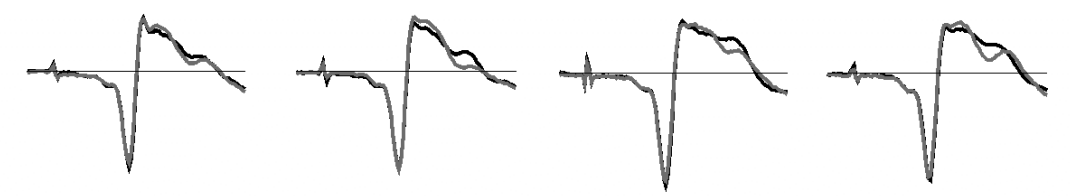

PASN20-20ms

PASN20-2.5ms

PASN20+Oms

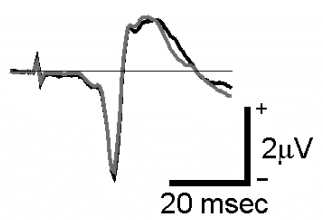

PAS $+5 \mathrm{sec}$

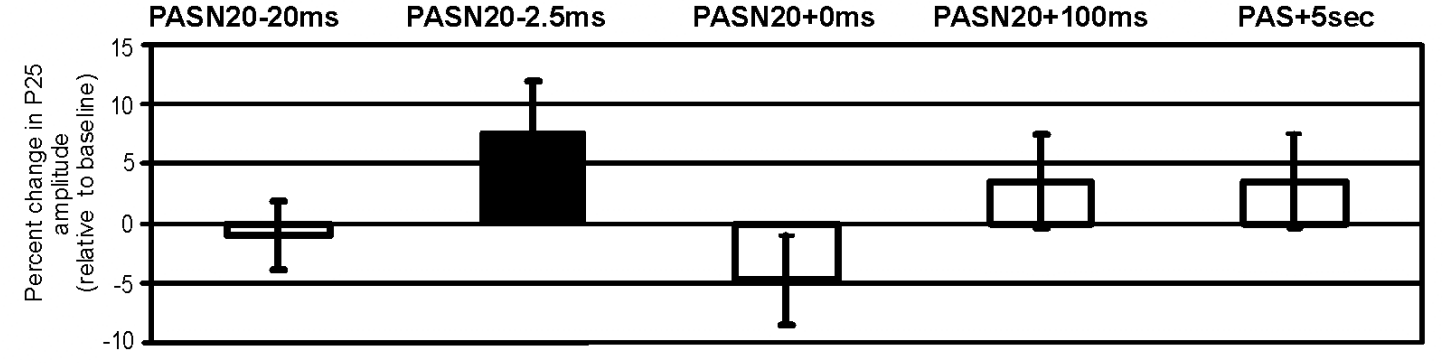

Figure 22. Changes induced by PAS in MN-SSEP waveforms. A. Grand averages of MN-SSEP (montage $\mathrm{CP3}-\mathrm{FCz}$ ) recorded before (black) and after (gray) PAS. B. Percentage change (relative to baseline) of the P25-component of the MN-SSEP. Amplitude of P25-component increased following PASN20-2.5ms (black bar indicates statistically significant change; $p=0.048$, paired t-test), in agreement with previous findings (Wolters et al., 2005). 


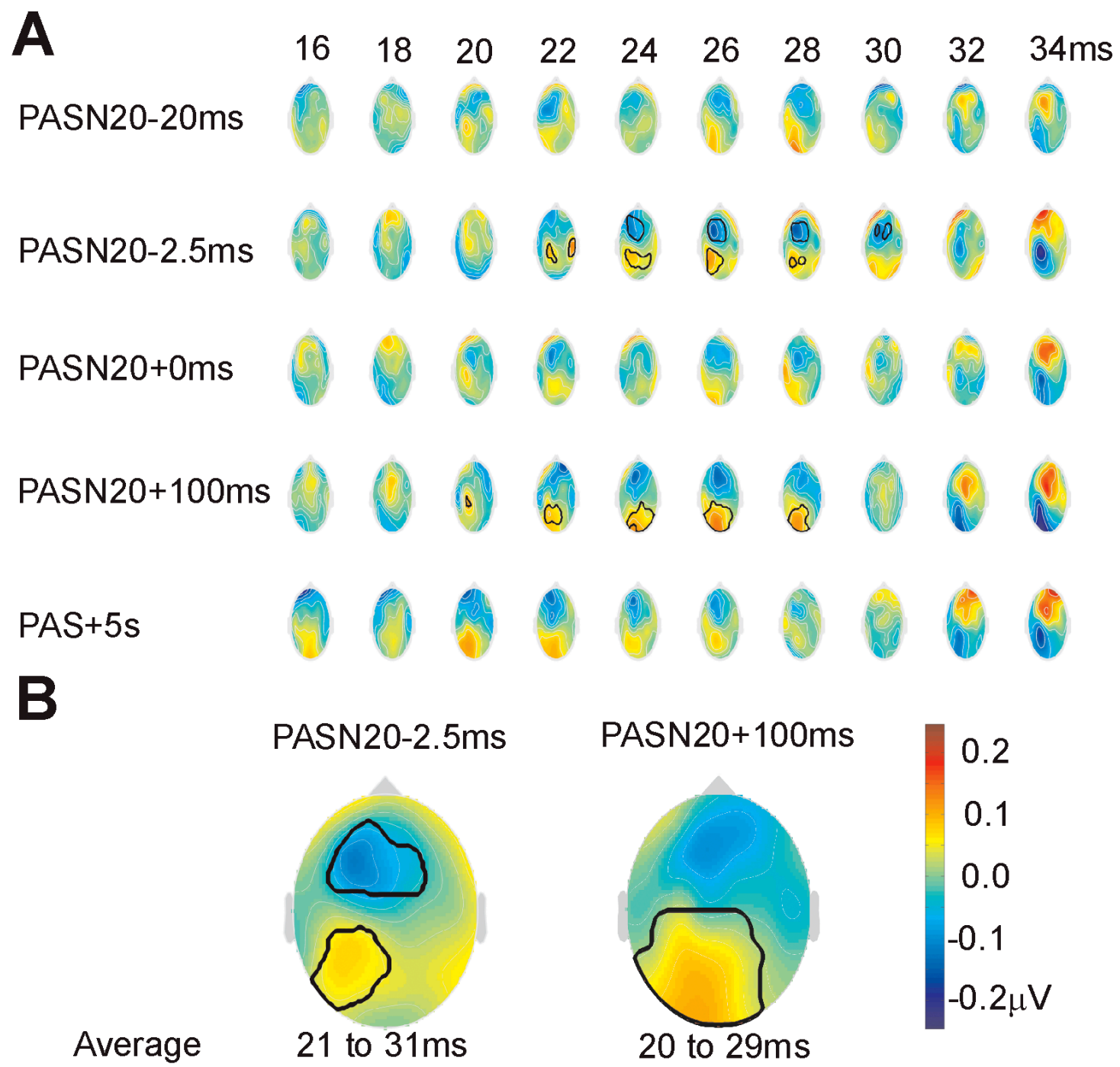

Figure 23. PASN20-2.5ms and PASN20+100ms significantly affect MN-SSEP amplitude between 21 and 31 ms post-stimulus.

A. Scalp maps of the differences between 'After PAS' and 'Before PAS' MN-SSEP recording. Each row presents results for a different PAS time interval condition and each column - a different time point after median nerve stimulation. The black contours encircle the significant clusters ( $p<0.01$, permutation based statistical analysis).

B. Average scalp maps of the significant effects of PASN20-2.5ms and PASN20+100ms. Scalp maps were averaged between 21 and $31 \mathrm{~ms}$ (PASN20-2.5ms) and 20 and $29 \mathrm{~ms}$ (PASN20+100ms) and compared statistically (clustering in space only). The black contours encircle the significant spatial clusters $(p<0.01)$. Note the differences between the scalp distribution of the effects of PASN20-2.5ms and PASN20-20ms 


\subsubsection{Fitting of equivalent dipoles reveals a difference between PASN20-2.5ms and PASN20+100ms effects}

The averaged over time scalp maps of the effects of PASN20-2.5ms and PASN20+100ms (Figure 23B) show similarities and differences between the two effects. In both cases there was a pattern consisting of a negative difference at the anterior part of the scalp and a positive difference at the posterior part. However, in the case of PASN20-2.5ms this pattern was more lateralized. To clarify the meaning of this dissimilarity between the two patterns single dipoles were fitted to the differences between "After PAS" and "Before PAS" MN-SSEP waveforms at the time window between 21 and $29 \mathrm{~ms}$ after the stimulus, separately for PASN20-2.5ms and PASN20+100ms. To allow the assessment of statistical significance of the difference between dipole positions in the two cases the fitting was performed using the Fieldtrip toolbox with a 4-shell ellipsoidal head model identical to that of BESA (Berg and Scherg, 1994b). Three hundred pairs of permuted datasets were created by randomly switching labels between PASN20-2.5ms and PASN20+100ms within individual subjects. Equivalent current dipoles were fitted to the averages of the permuted datasets and the resulting distances within each pair were compared to the distance between the two original dipoles. The results are shown in Figure 24. The dipole fitted to the PASN20+100ms difference was located more medial and posterior compared to the dipole fitted to the PASN20-2.5ms difference, which is consistent with the scalp maps of the two differences (Figure 23B). The nonparametric test showed that the original distance was significantly $(\mathrm{p}=0.017)$ larger than distances derived from randomly permutated data sets. 


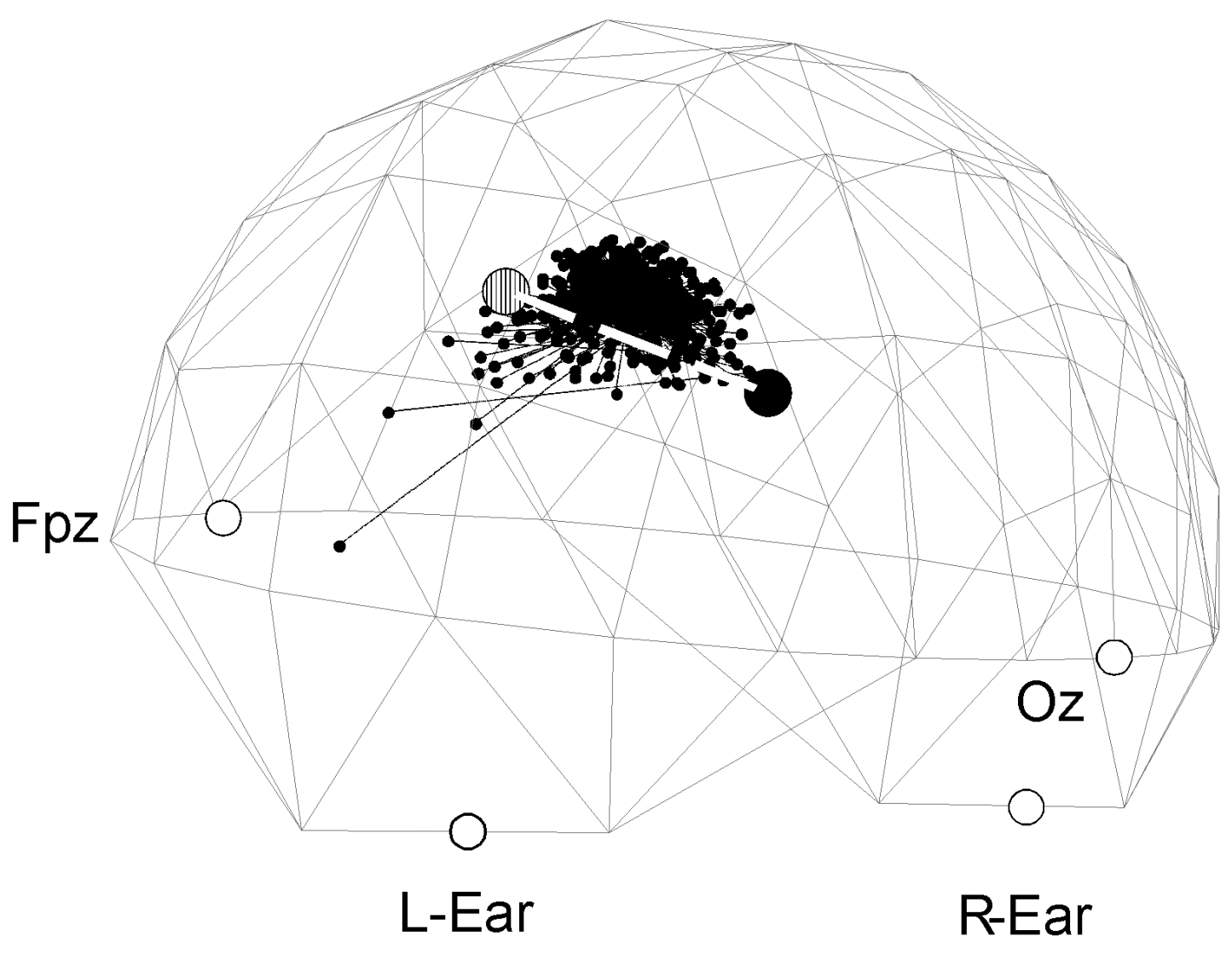

Figure 24. Fitting of equivalent dipoles reveals a difference between PASN20-2.5ms and PASN20+100ms effects.

Statistical comparison between the positions of dipoles fitted to PASN20-2.5ms (striped circle) and PASN20+100ms (full circle) effects using permutation based nonparametric method. The original distance was significantly $(p=0.017)$ larger than distances derived from randomly permutated data sets (small dots). 


\subsubsection{PASN20-2.5ms effect can be explained by an activity increase of the tangential $\mathbf{S 1}$ source}

To further characterize the cortical sources of PAS-induced differences in MN-SSEP the BESA SSEP source model (Buchner et al., 1995; Scherg et al., 2002) was used. Source waveforms (Scherg and Berg, 1996) were computed for "Before PAS" and "After PAS" conditions. Computations were performed separately for each subject for PASN20-2.5ms and PASN20+100ms, the two PAS conditions showing significant voltage differences at the scalp. The source waveforms were examined for four sources accounting for more than $90 \%$ of the MN-SSEP activity in most subjects - a brainstem source accounting for early activity in the medulla, a tangential source accounting for the activity in Brodmann area (BA) $3 \mathrm{~b}$ of $\mathrm{S} 1$, a radial source accounting for the activity of Brodmann area 1 and an 'orthogonal' source which accounts for the rest of the activity originating from the hand representation at S1, which is not explained by the tangential and the radial sources (Buchner et al., 1995; Ravazzani et al., 1995). Statistical comparison was performed between pre-PAS and post-PAS source waveforms of each source, separately for PASN20-2.5ms and PASN20+100ms, at the time window between 21 and $29 \mathrm{~ms}$ after stimulus, which is the temporal overlap between the statistically significant effects for the two conditions.. The results of this analysis are shown in Figure 25. At the time window of interest a significant difference $(\mathrm{p}<0.01)$ was found only between the pre-PAS and post-PAS waveforms of the tangential source for PASN20-2.5ms. This finding is consistent with the fact that the scalp projection of the tangential source (Figure 25A) is similar to the scalp map of the difference between "After PAS" and "Before PAS" for PASN20-2.5ms (Figure 23B). 
A
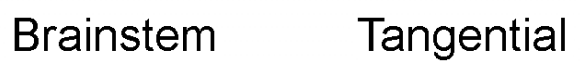

Radial

Orthogonal
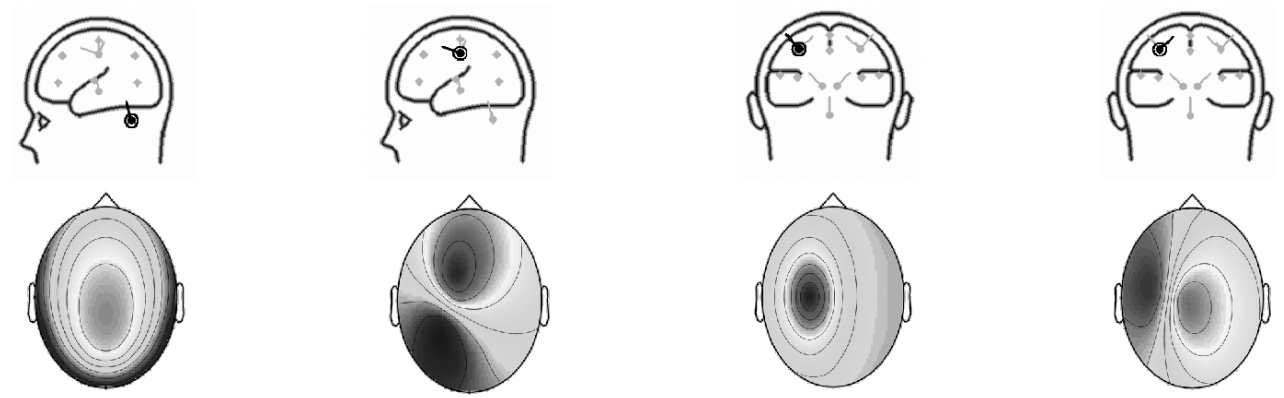

$\mathrm{B}$

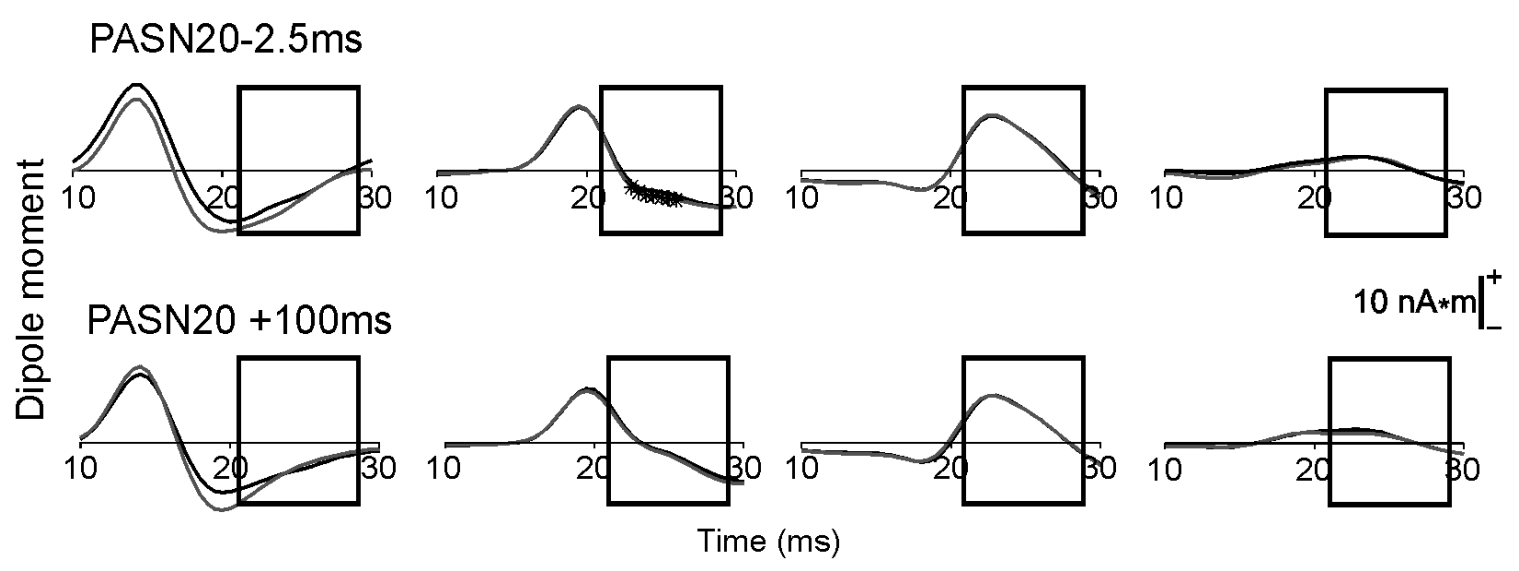

Figure 25. Analysis using BESA MN-SSEP source model shows that PASN20-2.5ms effect can be explained by an activity increase of the tangential $\mathrm{S1}$ source.

A. The brainstem dipole and the left $\mathrm{S} 1$ regional source (consisting of 3 dipoles) accounting for most of the MN-SSEP activity (Buchner et al., 1995). The upper row shows the positions of the dipoles and the lower row - their projections on the scalp.

B. Activity waveforms for the sources in A before (black) and after (gray) PAS. Statistical comparison was performed at the time window between 21 and $29 \mathrm{~ms}$, where significant differences were found on the scalp for both PASN20-2.5ms and PASN20+100ms (Figure 23A). The significantly different cluster is denoted with asterisks $(p<0.05$, nonparametric permutation test). The effect of PASN20+100ms cannot be explained by any of the sources in the model.

\subsubsection{PAS-induced alteration of two-point discrimination at the index finger}

Figure 26C shows the results of the two-point discrimination data analysis. Statistical significance was assessed using a mixed model separately for D2 and D5. The effect of PAS condition was significant for D2 $(\mathrm{p}=0.029)$ but not for D5 $(\mathrm{p}=0.427)$. Testing the differences between the means showed a significant improvement in two-point discrimination performance for D2 following PASN20-20ms ( $p=0.028)$. Following PASN20-2.5ms there was a weak trend for deterioration in performance in D2 not 
reaching significance $(p=0.207)$ and a significant improvement in performance for D5 $(\mathrm{p}=0.016)$. The latter finding should be treated with caution, however, since the effect of PAS as a whole for D5 was not significant. Other PAS conditions had no effects on the two-point discrimination performance.
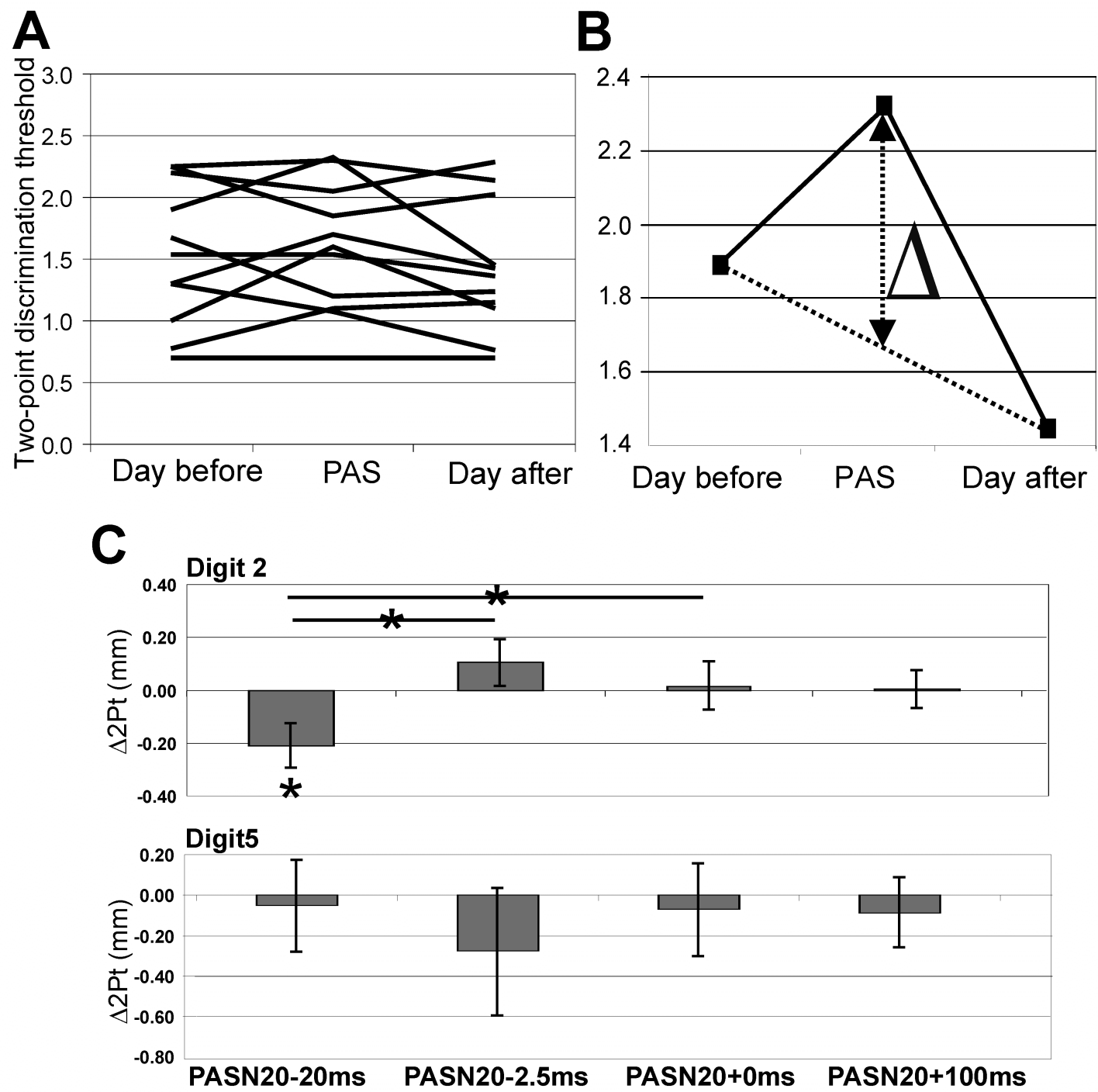

Figure 26. Statistical analysis of two-point discrimination data.

A. Two point discrimination thresholds of individual subjects for PASN20-2.5ms condition.

B. Schematic illustration of assessment of the change in two-point discrimination $(\Delta 2 P t)$ induced by PAS. $\triangle 2$ Pt represents the deviation induced by PAS from a linear trend between the values of the day before and day after PAS.

C. Effects of different PAS conditions on $\triangle 2 P t$ analyzed across subjects. The effect of PAS condition was significant for D2 $(p=0.029)$ but not for D5 $(p=0.427)$. For D2 there was a significant improvement in two-point discrimination performance following PASN20-20ms $(p=0.028)$. Following PASN20-2.5ms there was a weak trend for deterioration in performance in D2 not reaching significance $(p=0.207)$ and a significant improvement in performance for $D 5$ $(p=0.016)$. 


\subsubsection{Change in behavioral performance is correlated with modification of MN- SSEP amplitude by PASN20-2.5ms}

A nonparametric test was performed to test for a significant correlation across subjects between the $\Delta 2 \mathrm{Pt}$ parameter (summarizing the effect of PAS on two-point discrimination sensitivity in a single PAS condition) and the scalp map of the difference between MN-SSEPs "After PAS" and "Before PAS". The analysis was performed separately for D2 and D5 and for each PAS condition. A significant correlation was found only for D2 under PASN20-2.5ms. Two spatio-temporal clusters significantly correlated to $\Delta 2 \mathrm{Pt}(\mathrm{p}<0.02)$ were independently found at the same time between 28 and $32 \mathrm{~ms}$ after the stimulus. A positively correlated cluster was found at the anterior left part of the scalp and a negatively correlated cluster at the posterior left (Figure 27). The magnitude of the significant effect of PASN20-2.5ms on the MN-SSEP amplitude (21-31ms) described above was not correlated with $\triangle 2 \mathrm{Pt}$ in D2.

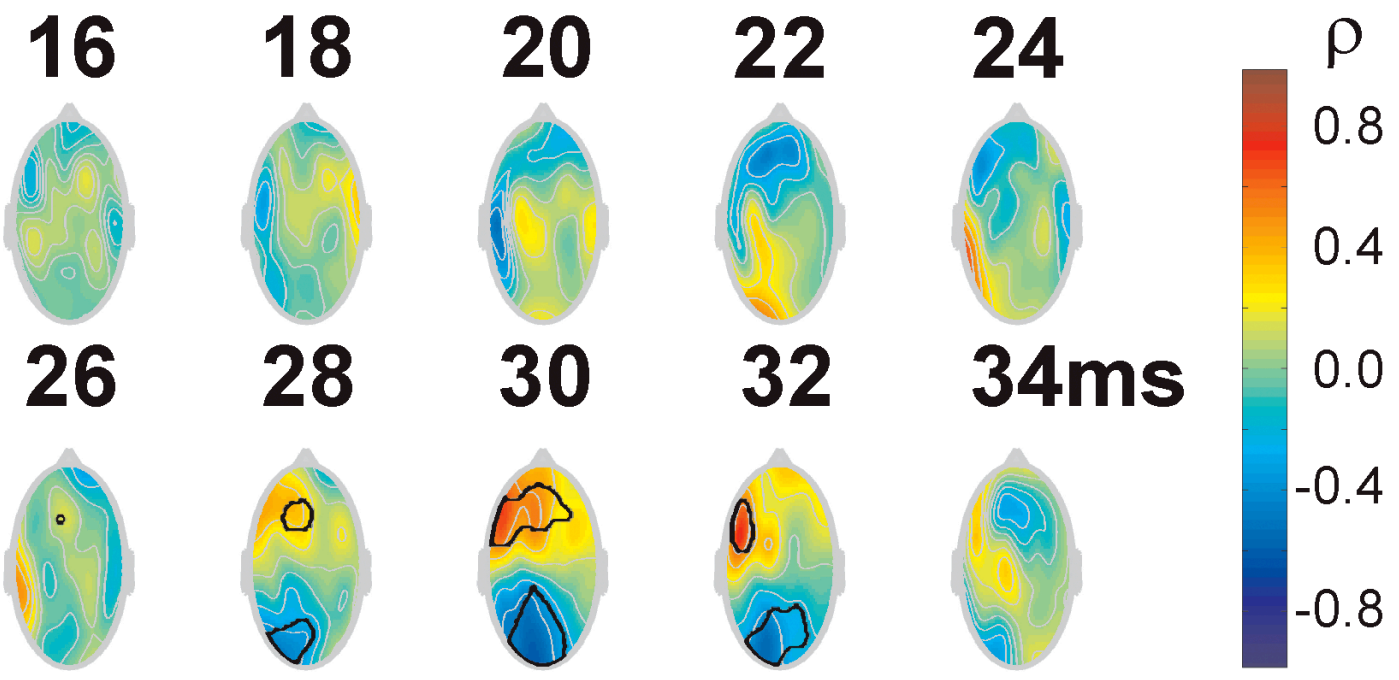

Figure 27. Significant correlation between the change in behavioral performance and modification of MN-SSEP amplitude by PASN20-2.5ms.

Correlation across subjects between the $\Delta 2 \mathrm{Pt}$ parameter and the scalp map of the difference between MN-SSEPs for "After PAS" and "Before PAS" recording (non-parametric test). For PASN20-2.5ms two spatio-temporal clusters (encircled by black lines) significantly correlated to $\Delta 2 \mathrm{Pt}(\mathrm{p}<0.02)$ were independently found by the method at the same time between 28 and $32 \mathrm{~ms}$ after the stimulus. 


\subsubsection{Discussion}

The present findings showed that PAS targeting the primary somatosensory cortex led to excitability changes as indicated by analysis of median nerve-somatosensory evoked potentials. As will be argued below, our observations provide novel support for the notion that neuronal excitability changes induced by Hebbian interaction of mechanoreceptive somatosensory signals with TMS-evoked neuronal responses are located in upper cortical layers of area $3 \mathrm{~b}$ of S1. In addition, the present results showed that PAS-induced excitability changes were associated with behavioral modifications.

\subsubsection{Effects of PAS employing mixed-quality afferent stimulation and asynchronous stimulation}

$\mathrm{PAS}+5 \mathrm{~s}$ did not induce significant effects on MN-SSEPs. This observation suggests that each stimulus alone is unlikely to generate consistent excitability changes. This conclusion is in agreement with our suggestion that the basis of the PAS effect at the cellular level is related to associative LTP, which requires near-synchronous activity.

PAS delivered with the neuronal events induced by both stimulation modalities at near-synchronicity induced a lasting modification in MN-SSEP amplitude that was dependent on the PAS interval. As reported previously (Wolters et al., 2005) the maximal effect was obtained for PASN20-2.5ms. Since the scalp voltage was more positive parietally and more negative frontally, clinical MN-SSEP recordings would show an increase in amplitude (Figure 22). One novelty of the present description of the PASN20-2.5ms effect is in the fact that it was revealed based on a data driven rather than hypothesis driven approach.

Following PASN20-20ms or PASN20+0ms, waveforms of MN-SSEP failed to confirm the previously observed decrease or increase (Wolters et al., 2005) at the peak around $25 \mathrm{~ms}$, when SSEP-data were viewed in a CP3-FCz montage or when non-parametric analysis was performed for these PAS-conditions. Exactly why PASN20-20ms and PASN20+0ms did not induce a significant depression or facilitation of SSEP components in the present study remains unclear. A possible 
explanation is that during the interventional stimulation the recording electrodes were left in place and hence the distance of the stimulation coil to the cortical surface was larger compared to our previous study. This different spatial arrangement may have led to reduced focality of the stimulated area (Ruohonen and Ilmoniemi, 1999; Thielscher and Kammer, 2002) with comparatively greater activation of adjacent cortical regions such as M1. Because, with coil placement over M1, PAS has previously been shown to enhance excitability in M1 (Wolters et al., 2003), the lack of facilitation of MN-SSEP with PAS20+0ms in the present study may reflect an interaction between S1 and M1 and between facilitatory and inhibitory effects. Such mutual interaction is implicated by a recent study combining fMRI with behavioral examination (Pleger et al., 2006). Similar considerations may apply for the lack of a PASN20-20ms induced effect. Additionally, protocols leading to depression of cortical excitability might be more susceptible to interference (Froc et al., 2000; Shulz et al., 2004; Froc and Racine, 2005) such as that implicated by the more complex recording arrangement. More studies are needed to explain this difference between the present and previous findings.

\subsubsection{Regional and laminar origin of PASN20-2.5ms - induced effects}

The generators of MN-SSEPs have been extensively studied with both invasive and non-invasive methods in humans and in animal models (for a review see (Allison et al., 1991)). While the P14-component of MN-SSEP is generated subcortically, the subsequent peaks are believed to be generated by the sensorimotor cortex contralateral to stimulation. According to the 'tangential + somatosensory radial' model which accounts for the majority of experimental findings in the field, there are two distinct cortical responses observed between 15 and $35 \mathrm{~ms}$. One complex, dubbed N20-P30 complex by some authors (Allison et al., 1991), originates at Brodmann area $3 \mathrm{~b}$ which is located at the posterior wall of the central sulcus (Geyer et al., 2001). Orientation of the cortical surface at this area results in current flow through anterior and posterior parts of the scalp and creates the typical 'tangential source pattern' of scalp voltages (Figure 25A). Another complex originates in Brodmann area 1, which is located at the crown of the postcentral gyrus (Geyer et al., 2001). It is termed P25N35 complex by some authors (Allison et al., 1991). In single-channel recordings, with the active electrode placed over the postcentral gyrus, the initial component of 
this complex corresponds to an inconsistently observed peak present at $\sim 22 \mathrm{~ms}\left(\mathrm{P} 22^{1}\right.$, (Mauguiere et al., 1999)). The current generated at this area flows through the scalp area located above the gyrus and this creates the 'radial source pattern' (Figure 25A). Due to these remarkably different scalp patterns the contributions of area $3 \mathrm{~b}$ and area 1 to the MN-SSEP can be separated despite the physical proximity of the generators.

If the response of each area is considered separately, it can be seen that both the N20P30 complex and the P25-N35 complex consist of similar sequences of "positivity" (as observed above the generating cortical surface) followed after a few milliseconds by "negativity" (Allison et al., 1991). Such responses have been extensively studied in animal models (for a review see (Mitzdorf, 1985)) using the current source density method. It was shown that both the "positivity" and the "negativity" are generated by current flow between cell bodies of pyramidal cells located in L4 and the apical dendrites of these cells reaching L2/3. The "positivity" is observed when the cell bodies and basal dendrites of the pyramidal cells are depolarized relative to the apical dendrites as a result of thalamic input. At a later stage the processing of the input by local recurrent circuits results in excitatory synaptic input to the dendrites of the same cells and as a result depolarization of the dendrites relative to the soma, reversal in the direction of the current flow and "negativity" is observed at the overlying cortical surface. This general sequence of events is common to different primary sensory areas and different species (Mitzdorf, 1985). In the specific case of MN-SSEP, the onset of this sequence at area 1 is delayed relatively to area $3 \mathrm{~b}$, presumably because the thalamic fibres projecting to these two areas differ in their conduction velocities (Allison et al., 1991).

${ }^{1}$ There is confusion in the literature between different naming conventions for MNSSEP peaks between 20 and $30 \mathrm{~ms}$. This confusion is partly due to different recording configurations and partly due to substantial variability of MN-SSEP shapes between healthy subjects. The positive peak of the N20-P30 complex is named by some authors P24, P25, or P27 (Garcia Larrea et al., 1992; Mauguiere et al., 1999). The positive peak of the P25-N35 complex is sometimes named P22 or P23 (Tsuji and Murai, 1986; Vanderzant et al., 1991; Garcia Larrea et al., 1992; Mauguiere et al., 1999). Especially confusing is the fact that the term P25 can refer to both complexes. The reason for this is that, although their cortical generators differ, it is not always possible to separate them in single-channel clinical MN-SSEP recording 
Between 21 and $29 \mathrm{~ms}$ after the stimulus, when the significant effects were observed in the present study, the activity observed at the scalp is a mixture of "negativity" originating from area $3 \mathrm{~b}$ and "positivity" originating at area 1 . Due to the orientation of area $3 \mathrm{~b}$ the actual "negativity" is observed anterior to the central sulcus while posterior to the central sulcus the scalp voltages are positive. Theoretically, PAS can affect area $3 b$, area 1 or both, but the fact that the effect is observed in this particular time window would lead to different interpretations with respect to the underlying synaptic mechanisms. If the cortical generator affected by PAS was that of area 1 (the radial source) then the increased response between 21 and 29 ms could most probably originate from strengthening of thalamo-cortical synapses at the cell bodies and basal dendrites of layer 4 pyramidal cells. Conversely, if the cortical generator affected by PAS was that of area $3 b$ (the tangential source) then the affected synapses would probably be the ones in L2/3 either at the apical dendrites of pyramidal cells or at interneurons activating these cells.

In the study of Wolters and co-workers (Wolters et al., 2005) it was not possible to discriminate between the two possibilities. In that study the MN-SSEPs were recorded by an electrode located above S1 with a reference electrode at the forehead. That montage picks up both tangential and radial sources and with a single channel it is not possible to separate the two. In the present study, it was shown that the PAS effect has a 'scalp signature' characteristic of the tangential source (Figure 23B). The source analysis in BESA confirmed that the tangential source activity was indeed increased, while the radial source remained unaffected. Therefore, it was possible to pinpoint the site of the PASN20-2.5ms effect both in terms of cortical area (area 3b) and in terms of cortical layer (L2/3). This conclusion is consistent with animal studies showing modulation of synaptic efficacy located in $\mathrm{L} 2 / 3$ in behaviorally relevant somatosensory plasticity (Allen et al., 2003; Celikel et al., 2004; Feldman and Brecht, 2005).

Although strengthening of the synapses in L2/3 is the most likely explanation for PASN20-2.5ms effect, an alternative explanation should be considered. An increase in current at the late "negativity" stage of the response indicates an increase in voltage gradient between the cell body and the apical dendritic tree of the pyramidal neurons. This gradient, however, can increase either as a result of stronger depolarization of the 
apical dendrites, as suggested before, or as a result of stronger hyperpolarization of the cell body. Indeed, the latter effect was suggested by observations of MN-SSEP evoked after administration of the GABA reuptake inhibitor tiagabine (Restuccia et al., 2002). However, increase in inhibition is unlikely to be the mechanisms of PASN20-2.5ms effect in the present study. In the case of PAS targeting the motor cortex it was shown that intracortical, presumably GABA-A receptor mediated inhibition remained unchanged after PAS (Ridding and Taylor, 2001; Stefan et al., 2002; Kujirai et al., 2006). Moreover, there is no evidence in the animal studies that STDP might leave excitatory synapses unchanged while inducing specific effects at GABAergic interneurons.

It seems noteworthy that the above conclusions, although in excellent agreement with the previous results of Wolters and co-workers (Wolters et al., 2005), were arrived at using a novel, completely data-driven approach. The traditional approach was based on comparing the amplitudes of known peaks in MN-SSEP waveform. Therefore, effects occurring not at peaks but during transition between the positive and the negative phase of the response could be missed. In the present study much of the PAS effect at the tangential source was indeed observed during such transition (Figure 25B).

The present study, therefore, illustrates the additional value of extended time window analysis over traditional methods of ERP analysis which are based on comparing peak amplitudes. The recently developed applications of nonparametric statistical methods to EEG/MEG data (Maris and Oostenveld, 2006) make it possible to look for effects with a data driven rather than hypothesis driven approach. The present study shows how this approach can be applied to a relatively simple problem where the underlying neural processes are constrained to a few testable alternatives. In more complicated cases where several cortical areas are simultaneously active and the activity on the scalp is a complex mixture of several effects, the nonparametric statistical analysis can help to tease out these effects by using different contrasts as done routinely in fMRI analysis (Poline, 2003). After the effects are separated, source analysis can clarify their cortical origin or at least formulate several alternative models to address this issue. 


\subsubsection{Effects induced by $P A S$ employing near-synchronous proprioceptive afferent stimulation (PASN20+100ms)}

PAS delivered at ISI $=\mathrm{N} 20+100 \mathrm{~ms}$ was found to have a significant effect. As outlined above, stimulation of the median nerve trunk at 300\% perceptual threshold releases a second, delayed afferent synchronous volley of somatosensory signals which carries information almost exclusively from muscle spindle endings. Neuronal events induced by the TMS pulse at $\mathrm{N} 20+100 \mathrm{~ms}$ are in close temporal proximity to the neuronal events induced by the afferents allowing for plasticity to occur through Hebbian mechanisms. However, because these signals are mainly proprioceptive they terminate in Brodmann area 3a (and 2) (Wiesendanger and Miles, 1982), rather than in BA 3b. Area 3a is the subdivision of the somatosensory cortex which receives most of proprioceptive signals (Wiesendanger and Miles, 1982). We consider it likely that this explains why the scalp topography of PASN20+100ms-induced changes was distinct from the effect induced by PASN20-2.5ms. The dipole fitted to the scalp map of the PASN20+100ms effect localized to the central sulcus area medial to the classical somatosensory representation of the hand and was oriented tangentially. This could be explained if we assume that that the source affected by PASN20+100ms is located in area $3 \mathrm{a}$ rather than in area 3b. Although some of area $3 \mathrm{a}$ is buried in the fundus of the central sulcus, a large part of it is located in the posterior wall of the postcentral gyrus (Geyer et al., 2001). Area 3a is located more medially than area 3b, due to its more rostral position along the pericentral region. Although, there are no established equivalent dipole models accounting for the activity arising in area $3 \mathrm{a}$ comparable to those of area $3 \mathrm{~b}$, the fact that proprioceptive evoked activity could be measured with magnetoencephalography (Druschky et al., 2003) indicates that at least some of this activity originates in tangentially oriented sources, which would be consistent with our findings.

\subsubsection{PAS-induced changes of behavior and their relationship to PAS-induced excitability changes}

Two-point discrimination changed significantly as a function of PAS-conditions (Figure 26C). Following PASN20-2.5ms, there was a trend for deterioration in twopoint discrimination performance in D2 and a significant improvement in performance for D5. In contrast, following PASN20-20ms, performance improved significantly in D2. Other PAS conditions had no effects on the two-point 
discrimination performance. In particular, perceptual performance remained unchanged with PASN20+100ms. Therefore, tactile performance changes were confined to near-synchronicity of mechanoreceptive signals with TMS-induced neuronal events whereas they were absent when near-synchronicity was confined to proprioceptive signals.

Effects induced by PASN20-2.5ms and PASN20-20ms were in opposite directions in the same digit (D2) and, for PASN20-2.5ms, led to opposite performance changes for digits inside (D2) or outside (D5) the targeted somatosensory representation. It appears highly unlikely that this distinctive pattern of behavioral changes emerged by chance. However, although suggestive, caution must be applied to relate this pattern of behavioral changes to the specific physiological characteristics of the PAS-protocol since our study had insufficient statistical power to yield significant results for all single effects, and no correlation emerged between the magnitude of PAS-induced SSEP-changes and behavior in an early time window. Therefore, confirmation by future studies is needed.

Our results may be compared to those obtained in previous studies investigating plasticity in the human somatosensory system and its effects on neuroimaging measurements and behavioral performance. Lasting perceptual improvements were induced by brief periods of $5 \mathrm{~Hz}$ rTMS over the cortical representation of the right index finger in S1 (Tegenthoff et al., 2005; Pleger et al., 2006), or by tactile costimulation applied to the tip of the index finger (Pleger et al., 2003). Functional MRI revealed an enlargement of the index finger representation in S1 whose magnitude was linearly correlated with the individual stimulation-induced perceptual improvement as assessed by two-point discrimination thresholds (Pleger et al., 2003; Tegenthoff et al., 2005) or frequency discrimination (Pleger et al., 2006). However, although changes in the orientation of equivalent dipoles were noted to correlate with perceptual performance (Pleger et al., 2001), direct comparisons between changes in the magnitude of early SSEP components and changes of perceptual performance have not been reported in these studies. Bidirectional changes in two-point discrimination thresholds were induced by intermittent high-frequency $(20 \mathrm{~Hz})$ and continuous low-frequency $(1 \mathrm{~Hz})$ tactile stimuli, respectively, to the tip of the index finger. Peripheral high frequency stimulation (HFS) induced a lowering of tactile discrimination thresholds, indicating improved tactile acuity in the stimulated 
fingertip, while low frequency stimulation (LFS) resulted in an impaired performance (Ragert et al., 2005). Because HFS and LFS are known to induce LTP and LTD, respectively, in animals, these observations may support a view that performance gains rely on LTP-like mechanisms while induction of LTD-like mechanism is associated with loss of perceptual abilities. However, Hodzic and co-workers found that, while tactile discrimination was improved after peripheral tactile co-activation, frequency discrimination was impaired. This indicated that stimulation protocols that lead to an enhancement of cortical activation may be associated with behavioral impairments in certain perceptual tasks (Hodzic et al., 2004). Therefore, equating LTP with performance gains, and LTD with performance impairments, may be overly simplistic.

In the present study an improvement of two-point discrimination occurred after PASN20-20ms in contrast to the depression of cortical excitability based on the previous study. However, a tendency for deterioration of two-point discrimination occurred after PASN20-2.5ms which caused enhancement of excitability. There is no a priori reason for performance improvement to be linked tighter to LTP than to LTD because both contribute to the coding of information in the brain (Martin et al., 2000). Conceivably, generation of LTD-like plasticity may help to focus activity (Nelson and Turrigiano, 1998) evoked by the probing two-point stimulus, while LTP-like plasticity leads to decline of performance via spatial blurring of activity. In the case of PAS with $\mathrm{MN}$ stimulation multiple fibers innervating the palm of the hand and fingers are stimulated within a small window of near synchrony with the cortical area representing the hand. This may lead to non-specific strengthening of synapses and thus to increasing the overlap between neighboring receptive fields, reducing discrimination ability.

As mentioned above for PASN20-2.5ms, the change in sensitivity of D5 was opposite to that of D2. This observation is reminiscent of organizational principles of surround suppression in the visual system (Carandini et al., 2002; Webb et al., 2005). Interestingly, stimulation-induced LTD-like changes of pain perception in the targeted skin region were surrounded by heterosynaptic LTP-like facilitation in adjacent skin regions (Klein et al., 2004). In animal studies, potentiation of one set of synapses has been shown to lead to heterosynaptic depression of nearby synapses and vice versa 
(Andersen et al., 1977; Lynch et al., 1977; Dunwiddie and Lynch, 1978; Scanziani et al., 1996; Royer and Pare, 2003).

The strongest effect on MN-SSEP was found for PASN20-2.5ms. However, as mentioned above, there was no correlation between the early significant EEG activity at $21-31 \mathrm{~ms}$ and tactile discrimination as assessed by two-point discrimination. In contrast, a highly significant correlation was found between the change in two-point discrimination threshold and MN-SSEP scalp map difference between 28 and $32 \mathrm{~ms}$ after the stimulus. Again, this effect was found with a data-driven approach and there is substantial evidence in favor of its physiological origin. The effect consists of two clusters, a cluster at the anterior left part of the scalp and a cluster at the posterior left part of the scalp. Because the significance of the clusters was assessed independently, the probability of observing such a pattern of two clusters at the same time by chance is extremely low. The pattern created jointly by these two clusters is similar to scalp voltage patterns created by tangential sources located near the central sulcus. Therefore, the most likely explanation for it is that there is a source near the central sulcus projecting to both anterior and posterior parts of the scalp. However, since the correlation coefficient is not a linear function of the data, it is not possible to validate this conclusion by fitting a dipole to the correlation scalp pattern. Visually, the pattern seems to be broadly consistent with the tangential source of S1 or with a source medial to it. In the latter case, it may be of relevance that the time period when the effect emerged from behavior-EEG correlation (28-32 ms) overlaps only the very end of the time period when the significant effect on the scalp EEG-map was observed (21-31ms) and, in fact, even outlasts it. This observation could be explained if we assume that the 28-32 ms EEG-activity reflects neuronal activity in M1 rather than S1. Indeed, previous studies using multichannel MEG or intracerebral recordings have shown that median nerve stimulation induces activity in M1 medially to the hand representation at S1 (Huang et al., 2000; Balzamo et al., 2004). Importantly, this activity was shown to reach its peak about $4 \mathrm{~ms}$ later than the area $3 \mathrm{~b}$ source (Balzamo et al., 2004). As mentioned above, a recent study combining fMRI with behavioral examination (Pleger et al., 2006) showed that much of the perceptual improvement induced by $5-\mathrm{Hz}$ repetitive TMS over $\mathrm{S} 1$ was encoded in a change in effective coupling between S1 and M1. Our data are fully in accord with this view and are compatible with a contribution of M1 in stimulation-induced changes of perceptual performance. Resolution of the precise spatial origin of these correlative 
changes could probably be provided by synchronous recordings of fMRI and multichannel EEG.

\subsubsection{Conclusions}

The present results support the notion that Hebbian interaction of mechanoreceptive afferent signals with TMS-evoked activity may modify synapses located in superficial cortical layers in BA $3 \mathrm{~b}$, and may induce timing-dependent, topographically specific behavioral changes in the same somatosensory quality. Although further studies are needed, our findings challenge the concept of a simple equation of enhancement of synaptic efficacy and functional gain. 


\subsection{Changes in amplitude of motor evoked potentials are correlated with the power of EEG-oscillations over sensorimotor cortex.}

\subsubsection{Introduction}

The substantial variability in the amplitude of potentials evoked in muscles by transcranial magnetic (TMS) and electrical (TES) stimulation has long been known to be one of the basic properties of these so called motor evoked potentials (MEPs) (Brasil-Neto et al., 1992; Kiers et al., 1993; Woodforth et al., 1996). On the one hand this variability has been considered an obstacle for precise assessment of excitability and functional mapping of the primary motor cortex (M1) (Brasil-Neto et al., 1992; Thickbroom et al., 1999). On the other hand the variability itself, measured as standard deviation or coefficient of variation of the MEP amplitude, was used in several studies to assess changes in the functional states of the motor system under different conditions (van der Kamp et al., 1996; Moosavi et al., 1999; Pitcher et al., 2003). Moreover, the physiological origin of the MEP variability has not been fully clarified to date. In particular, it is not clear whether it originates at the cortical or subcortical level. The indicator of cortical influence on MEP variability is the possibility to affect both mean MEP amplitude and its variance by voluntary muscle contraction (Darling et al., 2006) and motor imagery (Izumi et al., 1995; Rossini et al., 1999). MEPs evoked by anodal TES (which excites cortical output elements directly, downstream of the influence of intracortical elements) are much less variable than TMS-evoked MEPs (Amassian et al., 1990). This is further evidence for a largely cortical origin of variability. Conversely, Magistris et al. (Magistris et al., 1998), using a special stimulation protocol they developed, found that TES and TMS can achieve depolarization of all, or almost all, spinal neurons innervating a target muscle and that the variability from one stimulus to another is mostly due to variability of the desynchronization occurring within the corticospinal tract or at the level of the spinal motoneuron. However, no explanation as to why this proposed desynchronisation is so variable between trials was proposed and therefore these results do not rule out cortical involvement.

Variability of neural responses to identical stimuli has been addressed before in the visual system (Arieli et al., 1996). It was shown that the ongoing cortical activity can significantly influence the response pattern seen in the cortex with voltage sensitive 
dye imaging. In anesthetized animals, patterns very similar to specific responses to oriented sensory stimuli can arise over extensive cortical areas due to spontaneous activity alone, in the absence of any stimulus (Kenet et al., 2003). If a similar phenomenon occurs in the sensorimotor system, it can perhaps explain the spontaneously varying excitability of M1. By analogy with the visual system these spontaneous activity patterns could be expected to be similar to the evoked patterns associated with movement. Electroencephalography (EEG) is one of the ways to observe such patterns. In particular, decrease in spectral power in alpha and beta range in the period between $-1.5 \mathrm{sec}$ and $0.5 \mathrm{sec}$ relative to movement onset, termed event related desynchronization (ERD), has been described (Leocani et al., 1997; Pfurtscheller and Lopes da Silva, 1999). ERD is followed by an increase in power in the same sensors and frequencies relative to pre-movement baseline, termed event related synchronization (ERS). ERD was suggested to be associated with activation of the sensorimotor circuit whereas ERS - with its return to the idling state (Leocani et al., 1997).

The present study was aimed at testing the hypothesis that similar EEG spectral patterns might be associated with and have a predictive value for MEP amplitude.

\subsubsection{Methods}

\subsubsection{Subjects}

Experiments were performed on 8 healthy volunteers ( 4 men, 4 women), aged 21 to 44 (mean $26.6 \pm 7.0$ years) with normal results on neurological examination, who gave their written informed consent. All subjects were right-handed, by their own statement. The protocol conformed to the principles of the declaration of Helsinki and was approved by the Ethics committee of the University of Würzburg.

\subsubsection{MRI scan}

Anatomical magnetic resonance imaging (MRI) scans were obtained from 5 of the subjects for subsequent use for TMS navigation and EEG source imaging. Magnetic resonance tomography was performed using a Magnetom Vision 1.5-Tesla-Scanner (Siemens, Erlangen, Germany) utilizing a circular standard head coil following a 
routine imaging protocol. A conventional axial T1-weighted sequence was performed using a slice thickness of $1 \mathrm{~mm}$.

\subsubsection{Stimulation}

Transcranial magnetic stimulation (TMS) was performed using a flat figure-eight shaped magnetic coil (diameter of each wing: $70 \mathrm{~mm}$ ) connected to a Magstim 200 magnetic stimulator (Magstim, Whitland, Dyfed, UK). The coil was held tangential to the skull with the handle pointing backward and laterally at an angle of $\sim 45$ deg to the sagittal plane.

\subsubsection{Recording}

Electroencephalographic (EEG) signals were recorded from the scalp with 64-channel Quickamp system (Brain Products GmbH, Munich, Germany). The electrodes were located at extended 10-20 system locations and fixed on the subject's head with an elastic cap (EASYCAP GmbH, Herrsching-Breitbrunn, Germany). Electrode positions and anatomic reference points were measured for each subject with a $3 \mathrm{D}$ navigation system (Brainsight, Rogue research, Montreal, Canada). The brain signals were average referenced in hardware, filtered between 0 and $560 \mathrm{~Hz}$ and sampled at $2000 \mathrm{~Hz}$. Surface electromyographic (EMG) activity was recorded from the right abductor pollicis brevis muscle (APB) using the same recording system with the active electrode mounted on the muscle belly and the reference electrode placed over the base of the metacarpo-phalangeal joint of the thumb. In 6 of the 8 experiments EMG was also recorded in parallel by an additional system to provide auditory feedback to the subjects and assist their relaxation during the experiment. This feedback system used $\mathrm{Ag}-\mathrm{AgCl}$ electrodes (Fischer Medizintechnik, Nürnberg, Germany). The recorded signal was amplified (CED1902 amplifier, Cambridge Electronics Design, Cambridge, UK) and fed to a loudspeaker.

\subsubsection{Experimental procedures}

The principal purpose of all experiments was to identify EEG activity patterns predictive of the magnitude of TMS-evoked MEPs. 


\subsection{Search for motor hot spots and threshold measurement}

Subjects were seated in a comfortable chair. The optimal position of the magnetic coil for eliciting motor evoked potentials (MEP) in the right APB, termed "motor hot spot", was assessed over the left motor cortex using a moderately suprathreshold stimulation intensity. The coil position during the search for the motor hot spot was continuously monitored with a 3D navigation system registered to the subject's MRI scan if available. In cases when the subject was not scanned prior to the experiment ( $\mathrm{N}=3$ subjects), a scan of another subject with the most similar head dimensions was used. When the motor hot spot was found, the optimal position and orientation of the TMS coil for eliciting MEPs at this spot was marked directly on the scalp with a softtip pen and digitally stored in Brainsight software.

Following application of the EEG cap and reduction of the impedance of the electrodes to below $5 \mathrm{k} \Omega$, the resting motor threshold (RMT) at the motor hot spot was measured. RMT was defined as the magnetic stimulator intensity needed to produce a response of at least $50 \mu \mathrm{V}$ in the relaxed APB in $50 \%$ of the trials (resolution of $1 \%$ of the maximal stimulator output). The RMT value was determined using the Maximum Likelihood Threshold Hunting (MLTH) procedure (Awiszus, 2003). In this procedure TMS pulses are delivered at a frequency of $<0.2 \mathrm{~Hz}$. Stimulation intensities are computed by the MLTH routine so that the outcome of the stimulation would be the most informative for threshold determination at each stage. The outcome of each trial (success or failure to evoke a suprathreshold MEP) is provided to the routine as input. After several trials the routine produces an initial RMT estimate which improves with each subsequent trial. We continued this procedure until it converged, i.e. the RMT estimate varied within less than $1 \%$ of the stimulator output in several consecutive trials. The RMT was $45.9 \pm 4.1 \%$ of the maximal stimulator output. On average $23.9 \pm 5.5$ TMS pulses were necessary to achieve convergence. Complete muscle relaxation was monitored by examining the MEP waveform at the baseline period and if necessary audio-feedback was used to help the subjects monitor their relaxation. 


\subsection{Magnetic stimulation and MEP recording}

Subsequent to the determination of RMT, in each subject on average $514 \pm 5$ stimuli were delivered to the motor hot spot at an intensity of $115 \%$ RMT. The stimulator was triggered by the experimenter using a foot pedal with inter-stimulus intervals of about $5 \mathrm{sec}$ (mean of subjects' means $6.9 \pm 1.3 \mathrm{sec}$ ). The purpose of manual triggering was to avoid expectation related activity by avoiding constant interval. In addition, the experimenter monitored the EEG recording and triggered the stimulator after artifact free recording was observed for several seconds in order to increase the number of trials suitable for analysis. The whole recording lasted about 2 hours and subjects were allowed to take 1-3 breaks (1.8 \pm 0.8$)$ of 5-10 min to rest.

\subsection{Self-initiated movements recording}

After completing the TMS stimulation and taking a short break $(\sim 10 \mathrm{~min})$ the subjects were asked to perform self-initiated abduction movements of the right thumb approximately every $5 \mathrm{sec}$. They were instructed not to count, but move spontaneously whenever they felt the urge to move. EEG and EMG were recorded. The recordings lasted for about 15-20 $\mathrm{min}$ (18.8 \pm 1.4$)$ and approximately 200 $(199 \pm 60)$ movements were recorded.

\subsubsection{EEG analysis}

The EEG data were processed using BESA EEG analysis software (MEGIS Software $\mathrm{GmbH}$, Gräfelfing, Germany) and the Fieldtrip open source Matlab toolbox (http://www.ru.nl/fcdonders/fieldtrip/) developed at the F.C. Donders Centre for Cognitive Neuroimaging.

\subsection{Analysis of pre-TMS EEG recordings}

At the first stage of analysis trials free of artifacts at the $2 \mathrm{sec}$ preceding the TMS stimulus were selected using the BESA artifact rejection interface (Scherg et al., 2004). Before testing for the presence of artifacts, the EEG was high-pass filtered (1.5 $\mathrm{Hz}, 6 \mathrm{db} / \mathrm{oct}$, forward filter). Forward filter was used in order to prevent the TMS 
artifact from affecting the pre-TMS EEG. In cases when multiple eye blink artifacts were present in the data they were corrected before the artifact scan (Berg and Scherg, 1994a) in order to prevent rejection of trials contaminated only by eye blinks. Trials were considered artifact and excluded from averaging if they satisfied one of the following two conditions after filtering and eye blink artifact correction. (1) The amplitude difference between the largest and the smallest sample within the trial in any channel exceeded $200 \mu \mathrm{V}$. (2) The voltage difference between two adjacent samples in any channel exceeded $75 \mu \mathrm{V}$. These settings resulted in rejection of $3.8 \pm 3.1 \%$ of the trials.

The subsequent processing was done using Fieldtrip. MEPs were examined conditional upon artifact-free pre-stimulus EEG. EMG was high-pass filtered at 1.5 $\mathrm{Hz}$ (Rossini et al., 1994). The MEP amplitude was defined as the difference between maximum and minimum EMG voltage at the segment between 20 and $50 \mathrm{~ms}$ after the stimulus.

The trials were then sorted based on the log amplitude of the MEP and two groups were selected - the $1 / 3$ of the trials with the lowest amplitude and $1 / 3$ with the highest amplitude. The intermediate 1/3 was not analyzed. The number of trials analyzed was $165 \pm 5$ for each of the two conditions.

For the two selected groups of trials EEG between -2005 and $-5 \mathrm{~ms}$ relative to the stimulus was downsampled to $250 \mathrm{~Hz}$ and linear trend was removed from the entire epoch for each channel.

Spectra were computed over the entire $2 \mathrm{sec}$ segment using a multi-taper method (Mitra and Pesaran, 1999) between 0 and $45 \mathrm{~Hz}$ with an effective smoothing window of $\pm 2 \mathrm{~Hz}$.

Statistical comparison of log-spectra was performed using the non-parametric permutation-based method implemented in Fieldtrip (Maris and Oostenveld, 2006).

In order to examine the time course of the effect, the EEG recorded at the C3 channel was filtered in two bands defined based on the results of the statistical analysis of the spectra between 6.5 and $11.5 \mathrm{~Hz}$ and between 17.5 and $39.5 \mathrm{~Hz}$. The root mean square 
(RMS) of all trials was computed for each subject in the time window -2 to $0 \mathrm{sec}$ relative to the movement. In order to remove residual oscillations not suppressed by averaging without distorting the timing of slower power changes the RMS curves were smoothed using local regression (Loader, 1999) with the width of each smoothing neighborhood covering $10 \%$ of the data.. The grand average of the smoothed curves was then computed. To examine a longer time period preceding the TMS stimulus, the same analysis was repeated for a subset of trials where the artifact free $5 \mathrm{sec}$ preceding the TMS epoch was available. Artifact rejection was performed as described above. As a consequence of manual triggering, in some cases the interstimulus interval was shorter than $5 \mathrm{sec}$. In addition, other artifacts were sometimes present between -5 and -2 sec. Thus, fewer trials were available for this analysis than for other analyses described above ( $90 \pm 25$ for each condition).

\subsection{Analysis of EEG around voluntary movements}

The analysis of EEG recorded while subject performed voluntary movements was performed in a manner as similar as possible to the analysis of pre-TMS recordings taking into account the differences between the two data sets.

Movement onset times were defined based on the EMG using a BESA in-built routine. This routine was configured individually for each subject in order to achieve detection of all the movement related activity observed by eye as close to the onset as possible.

Only movements preceded by a movement-free interval of at least $4.5 \mathrm{sec}$ were selected for analysis except for one subject who performed the movements so frequently that not enough such trials could be collected. For this subject the required movement-free interval was set to $3.5 \mathrm{sec}$.

Trials containing artifacts at the period between -4 and $+0.5 \mathrm{sec}$ relative to the movement were rejected using exactly the same procedure as for pre-TMS EEG (see above). The number of trials analyzed was $124 \pm 23$.

Subsequent processing was conducted using Fieldtrip. Based on the literature (Leocani et al., 1997; Pfurtscheller and Lopes da Silva, 1999) we looked for 
movement related changes in the alpha band $(10-12 \mathrm{~Hz})$ and in the beta band (28-26 $\mathrm{Hz}$ ) in the $\mathrm{C} 3$ channel overlying the sensorimotor area contralateral to the movement. The EEG signal was band-pass filtered in the abovementioned bands and the root mean square (RMS) of all trials was computed for each subject in the time window -4 to $+2 \mathrm{sec}$ relative to the movement. In order to remove residual oscillations that were not suppressed by averaging without distorting the timing of slower power changes the RMS curves were smoothed using local regression (Loader, 1999) with the width of each smoothing neighborhood covering $10 \%$ of the data.. The grand average of the smoothed curves was then computed. Based on this grand average the baseline period was defined between -3 to -1 sec relative to the movement and movement period from -1 to $+1 \mathrm{sec}$ relative to the movement. The analysis of the multichannel EEG in these two windows was then performed with downsampling, spectrum computation and statistical comparison across subjects, exactly as described above for pre-TMS data.

\subsection{Scalp current density computation}

In order to compare the nature of the power differences seen in the pre-TMS data and in the movement data it would be of interest to know the cortical sources generating these differences. There is a variety of methods making it possible to compute the current source density in the cortex from EEG data recorded on the scalp, each based on different assumptions (Scherg and Berg, 1996; Pascual-Marqui, 1999; Gross et al., 2001). However, not all of these methods are suitable for computing the cortical current source density based on power and those that are suitable do not offer sufficient spatial resolution to distinguish between sources located in close proximity in the sensorimotor area adjacent to the central sulcus. We therefore applied a simpler method of scalp current density (SCD) (also known as surface Laplacian) computation (Hjorth, 1991). This method enables to achieve 'deblurring' of the projections of cortical generators on the scalp. The SCD was computed from the raw data prior to computing the spectra using the finite-differences method (Hjorth, 1991) as implemented in Fieldtrip. 


\subsubsection{Results}

\subsubsection{Differences between the spectra of EEG preceding high-and low- amplitude MEP trials.}

Figure 28 shows the MEP amplitude as a function of time over the course of the experiment for each subject. Note the rapid fluctuations in MEP amplitude and the fact that two stimuli adjacent in time may result in MEP amplitudes differing by orders of magnitude. On the other hand, the mean amplitude also fluctuated with time in most subjects, though no clear tendency for either increase or decrease was observed. Figure 29 shows the results of comparison between the spectra of EEG preceding the magnetic stimulus. Nonparametric paired samples test comparing the power spectra of the segments between -2005 and $-5 \mathrm{~ms}$ prior to the TMS pulse between one third of trials with highest MEP amplitude and the one third of trial with the lowest MEP amplitude for the frequency range 0 to $45 \mathrm{~Hz}$ revealed two large clusters where the power for high amplitude MEP trials was increased relative to the power for low amplitude MEP trials. The first cluster was in the frequency range 6.5 to $11.5 \mathrm{~Hz}$ (alpha range) $(\mathrm{p}<0.1)$ and the second was between 17.5 and $39.5 \mathrm{~Hz}$ (beta range) $(\mathrm{p}<0.05)$. Figure 29A shows the clusters in channel-frequency space. Figure 29B shows the scalp distribution of power for the frequency ranges encompassed by the two clusters. The highest significant difference was observed at the electrodes overlying the sensorimotor area on the stimulated side (i.e. contralateral to the stimulated muscle). There was also some difference at the corresponding electrodes on the other side (especially pronounced for the alpha range). Remarkably, there were no clusters where the difference in power was in the opposite direction (negative in the plot). Non-significant negative differences were only observed in the beta range at $\mathrm{T} 7$ and $\mathrm{T} 8$ channels and are most probably due to temporal muscle noise. 


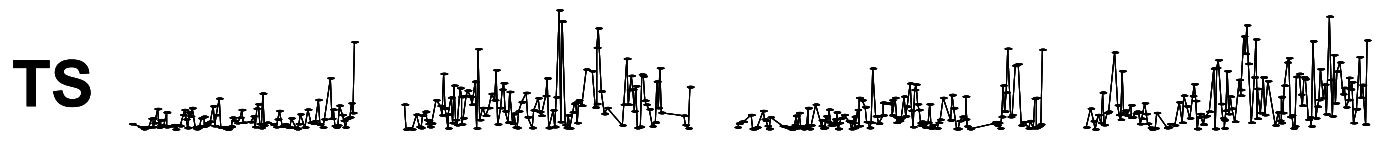

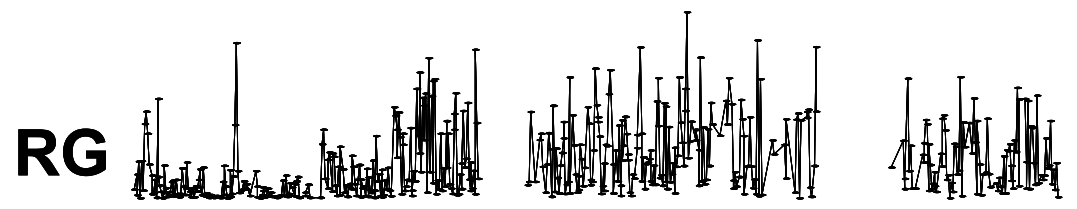

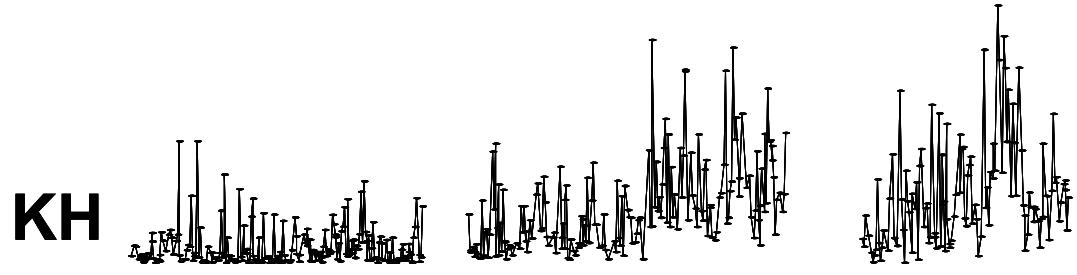

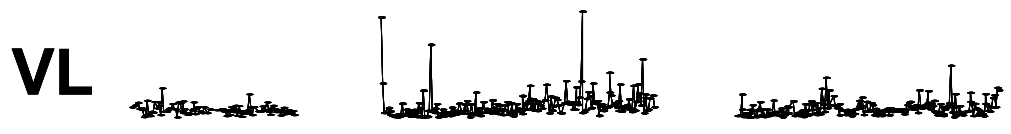

NW
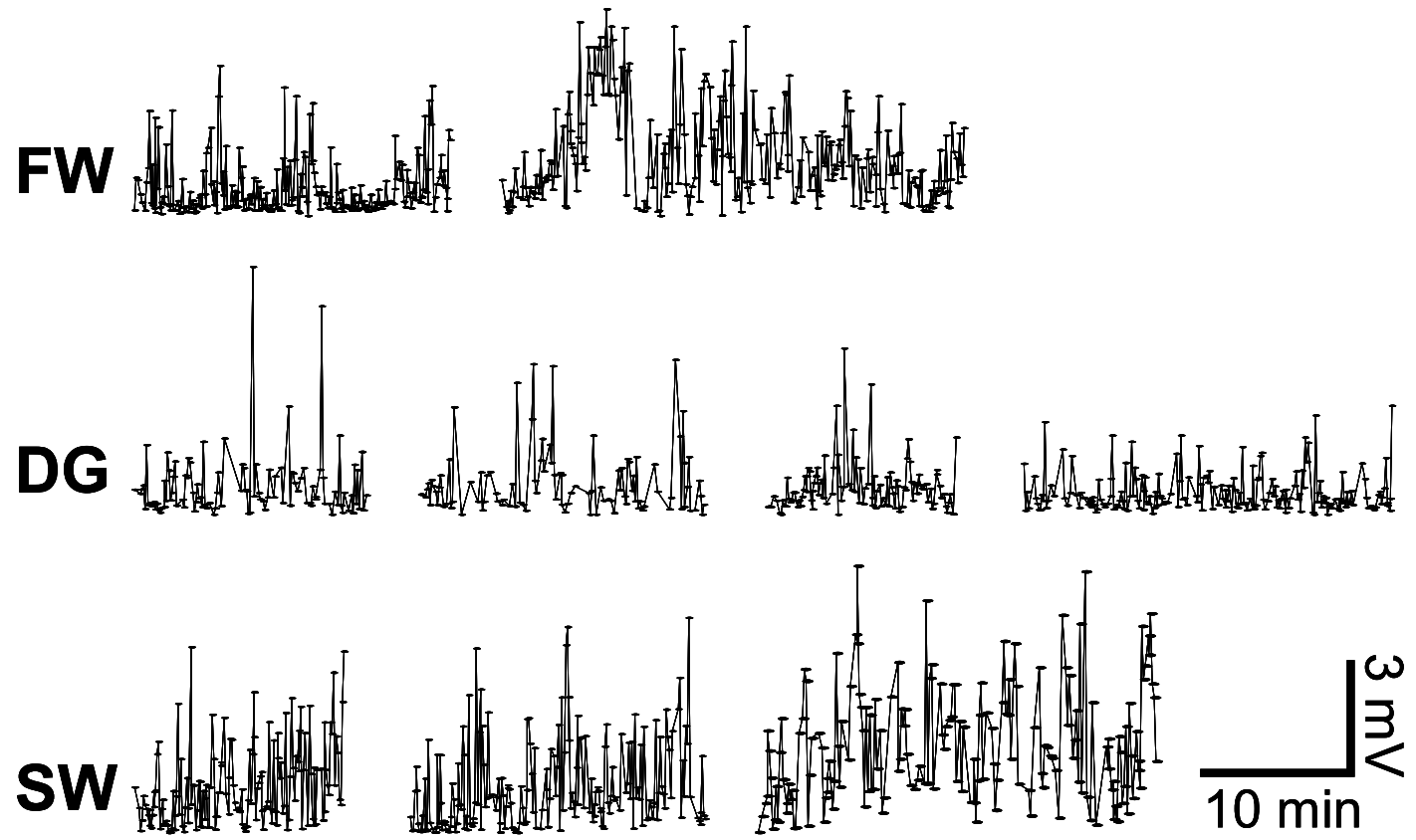

Figure 28. MEP amplitudes observed over the course of the experiment in each subject. The gaps represent the breaks during which the subject rested. 


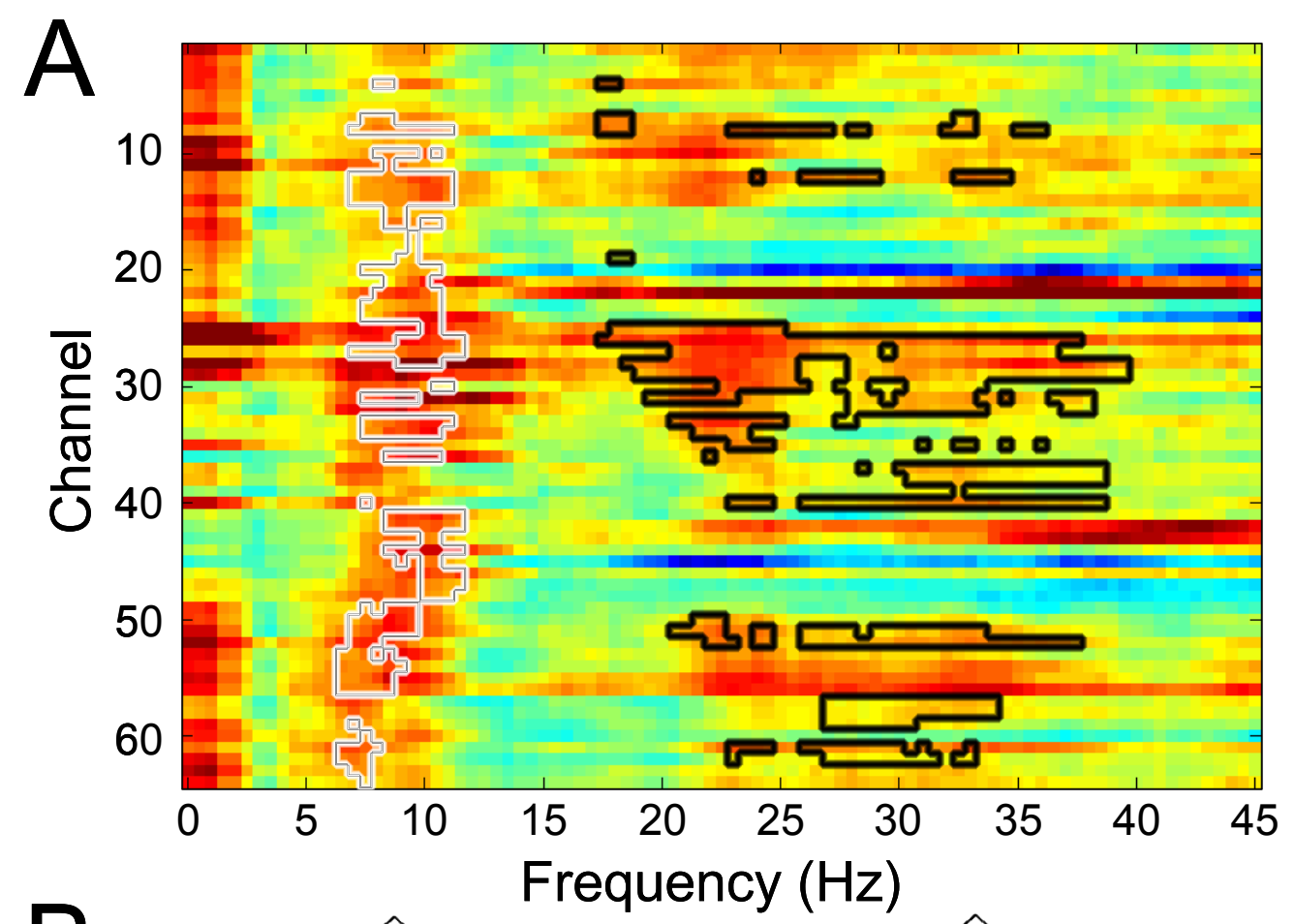

B

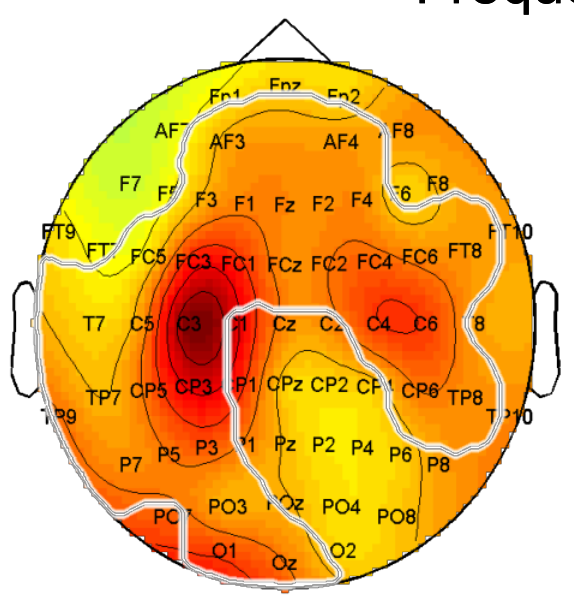

$6.5-11.5 \mathrm{~Hz}$

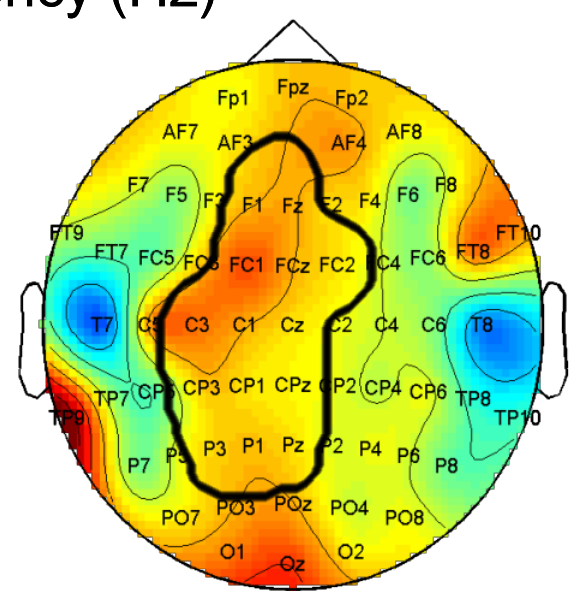

17.5-39.5 Hz

Figure 29. Differences in pre-stimulus EEG spectra between trials with high and low MEP amplitude. The time segment of two seconds preceding the TMS pulse was analyzed. The one third of the trials with the highest MEP amplitudes was compared to one third with the lowest MEP amplitudes. Non-parametric statistical analysis revealed two clusters (A): the cluster in the alpha range (white border, $p<0.1$ ) between 6.5 and $11.5 \mathrm{~Hz}$ and the cluster in the beta range (black border, $p<0.05$ ) between 17.5 and $39.5 \mathrm{~Hz}$. The clusters do not appear continuous in the figure since the order of the channels on the y-axis does not always represent their spatial proximity. In fact, the clusters are indeed continuous in both frequency and space. (B) The scalp power differences in the two frequency ranges corresponding to the clusters in A. Statistical analysis comparison with clustering in space revealed a significant cluster in each case $(p<0.01$ alpha, $\mathbf{p}<0.05$ beta). 


\subsubsection{Changes in spectral power associated with self-initiated movement}

The mean time interval between the self initiated thumb movements across the subjects was $6.0 \pm 1.4 \mathrm{sec}$. Movements not preceded by at least $4.5 \mathrm{sec}(3.5 \mathrm{sec}$ for subject NW) of a movement-free baseline period were excluded from analysis. Figure 30 shows the grand average of power changes in alpha and beta range around the movement onset recorded at channel C3. ERD was observed for both frequency ranges between -1 and $1 \mathrm{sec}$ relative to movement onset and this window was therefore chosen for further analysis of multichannel EEG. The window between -3 and $-1 \mathrm{sec}$ relative to movement onset was chosen as baseline. Figure 31 shows the results of EEG spectra comparison between the movement and baseline. Nonparametric paired samples test comparing the power spectra in the two windows for the frequency range 0 to $45 \mathrm{~Hz}$ revealed a single cluster $(p<0.001)$ where the power was decreased during movement (Figure 31A). This cluster covered both the alpha and beta frequency range $(7-35 \mathrm{~Hz})$. Although for some channels a gap can be seen at $15-20 \mathrm{~Hz}$ where no power decrease was observed, for other channels there was no gap at all and therefore the clustering algorithm used for the statistical analysis could not separate the effects in alpha and beta, as was the case for pre-TMS data. In order to compare the scalp patterns between the movement and baseline periods the power difference was averaged over the same two frequency ranges revealed in the analysis of pre-TMS data $-6.5-11.5 \mathrm{~Hz}$ and 17.5-39.5 $\mathrm{Hz}$ and nonparametric statistical comparison was performed in space only. In both ranges a single large negative cluster was found covering a large part of the scalp ( $<<0.01$ in both cases). The scalp maps of the difference were very similar between alpha and beta unlike in the pre-TMS case. For both alpha and beta the most significant difference was observed at the electrodes overlying the sensorimotor area contralateral to the movement. There was also a clear difference at the corresponding electrodes on the ipsilateral side. 


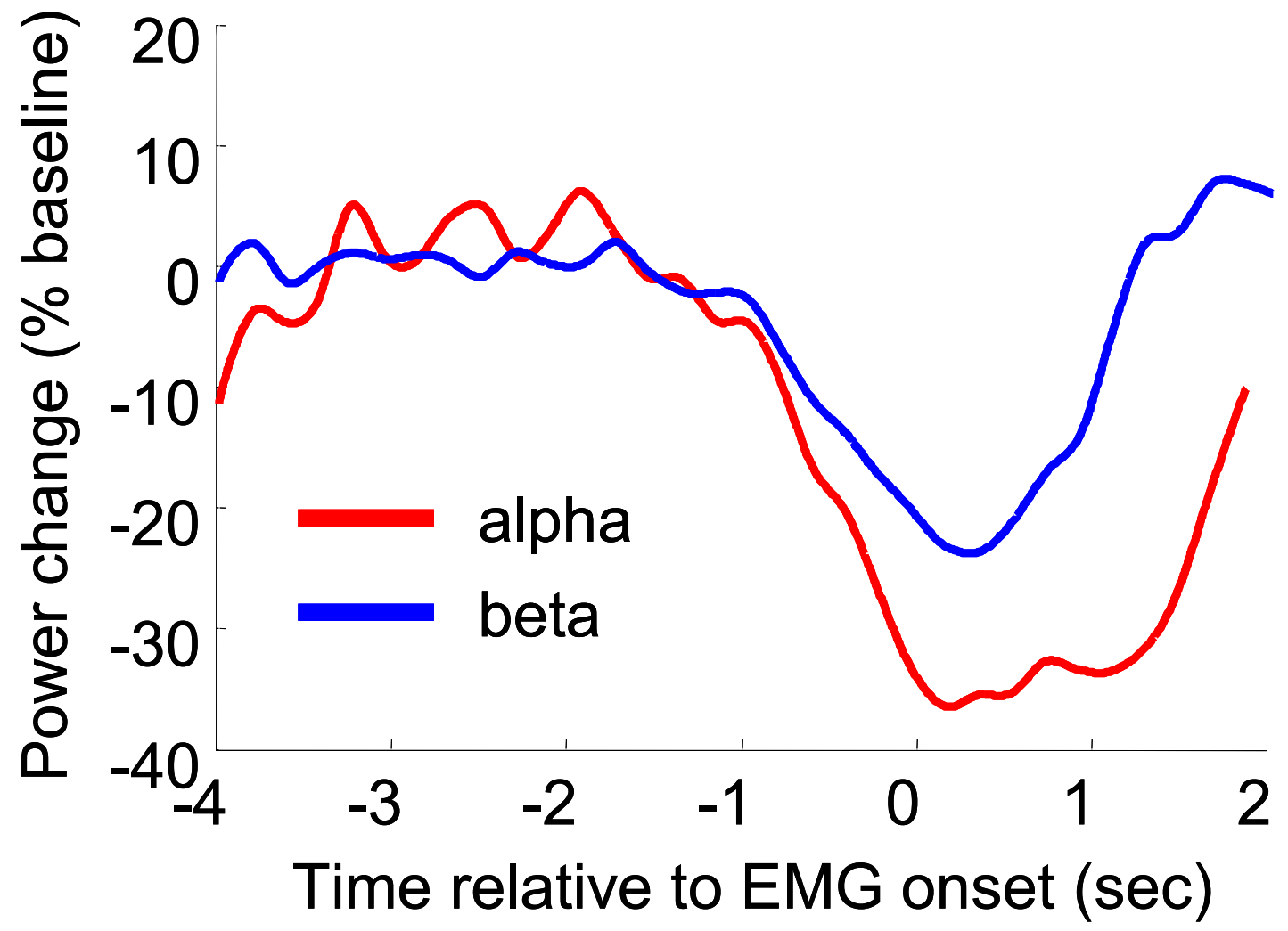

Figure 30. The time course of power changes surrounding self-initiated movement of the right thumb. The EEG recorded at the $\mathrm{C} 3$ electrode overlying the contralateral $\mathrm{M} 1$ was filtered in two frequency bands defined according to the literature: alpha band (10-12Hz) and beta band (28-26 Hz). The RMS of the filtered EEG was computed over trials, smoothed using local regression and grand averaged. ERD can be observed starting at -1 sec before the onset of the EMG activity. 


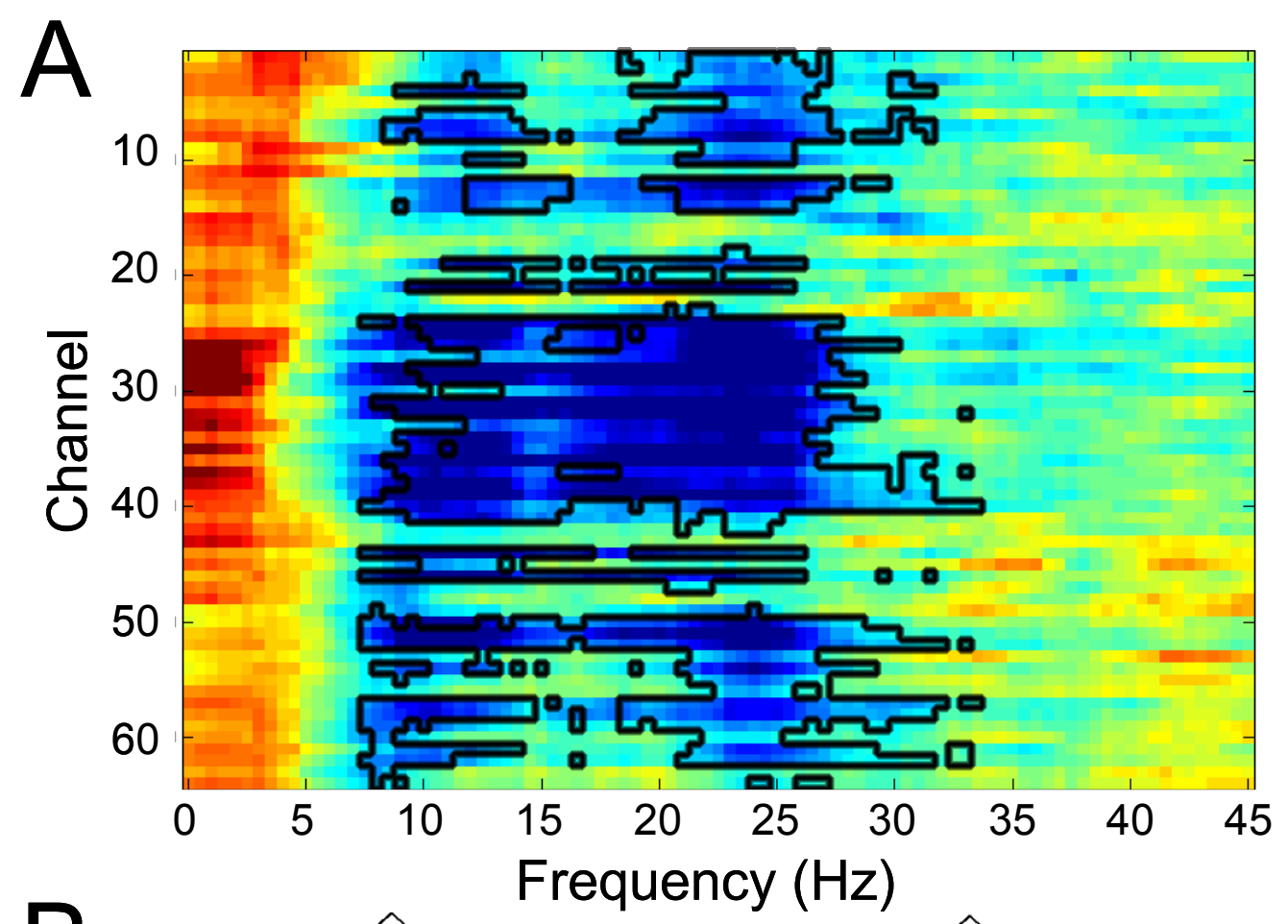

$\mathrm{B}$

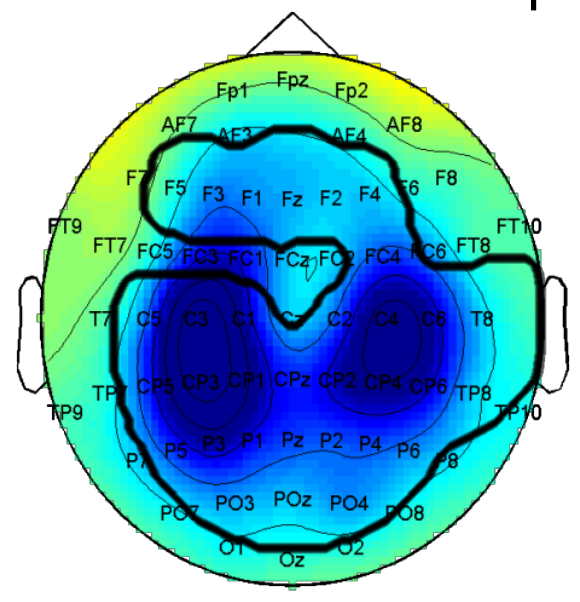

$6.5-11.5 \mathrm{~Hz}$

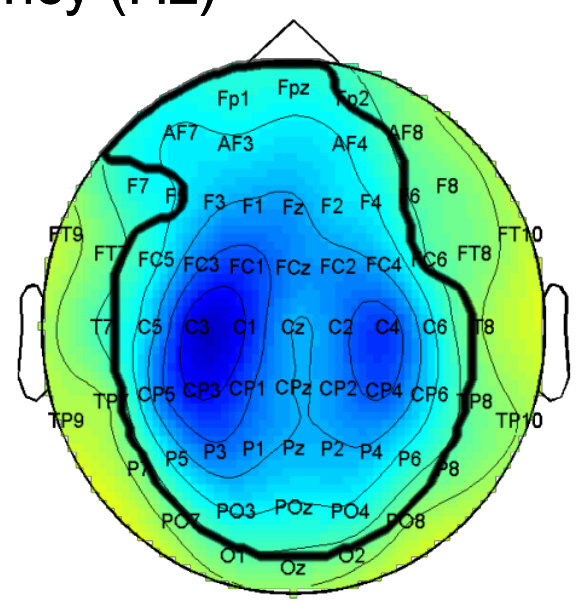

17.5-39.5 Hz

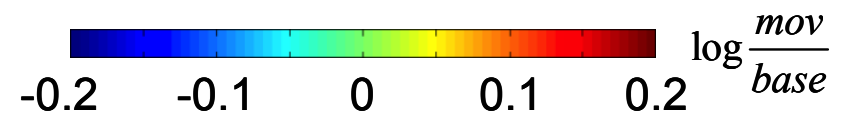

Figure 31. Comparison of the EEG recorded around the time of thumb movement to the baseline period. The baseline was defined as $\mathbf{- 3}$ to $\mathbf{- 1}$ sec relative to EMG onset and the movement period as -1 to 1 sec. (A) Non-parametric statistical analysis in frequency-channel space revealed a significant cluster between 7 and $35 \mathrm{~Hz}(\mathrm{p}<0.01)$. (B)The scalp power differences in the two frequency ranges corresponding to the clusters found for pre-TMS data. Statistical analysis comparison with clustering in space revealed a significant cluster in each case $(\mathbf{p}<0.01$ alpha, $\mathbf{p}<\mathbf{0 . 0 1}$ beta) 


\subsubsection{The time-course of power changes associated with increased MEP amplitude}

Figure 32 shows the power before the TMS pulse in the two bands defined by the nonparametric statistical analysis separately for high- and low- MEP amplitude trials. It can be seen that the power increase for the high- MEP amplitude trials is not limited to a short time-window preceding the magnetic stimulus but can be seen over the entire $2 \mathrm{sec}$ pre-stimulus window. The estimate of power changes over the $5 \mathrm{sec}$ preceding the TMS pulse (which is less reliable since it is based on fewer trials) shows that the difference between the two types of trial can be seen at least up to $4 \mathrm{sec}$ before the stimulus.
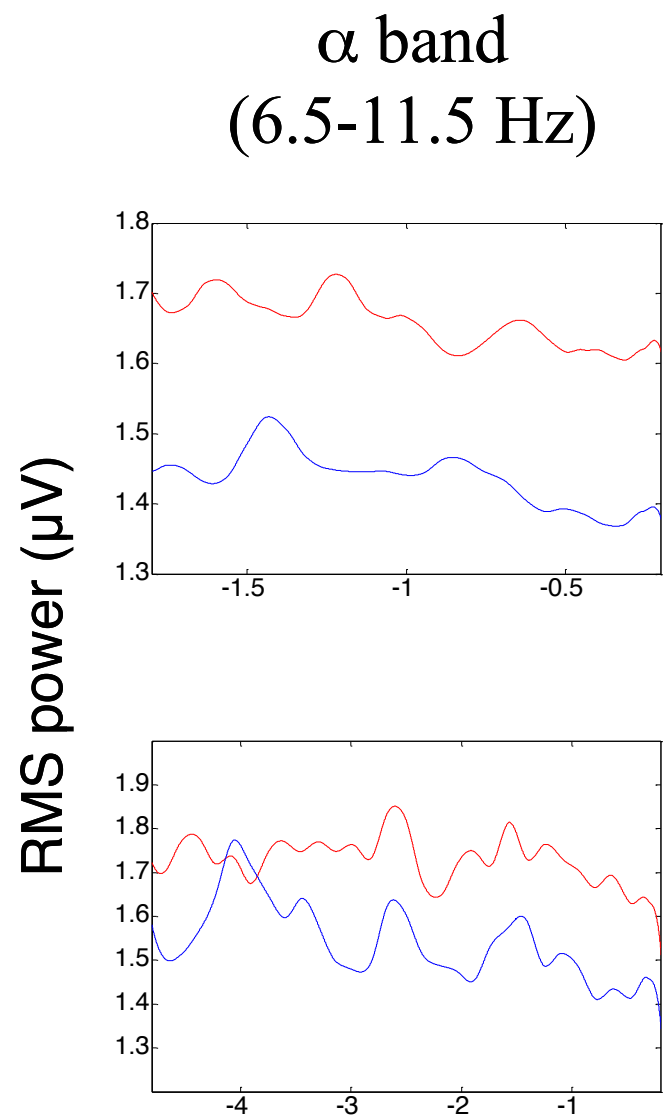

$\beta$ band
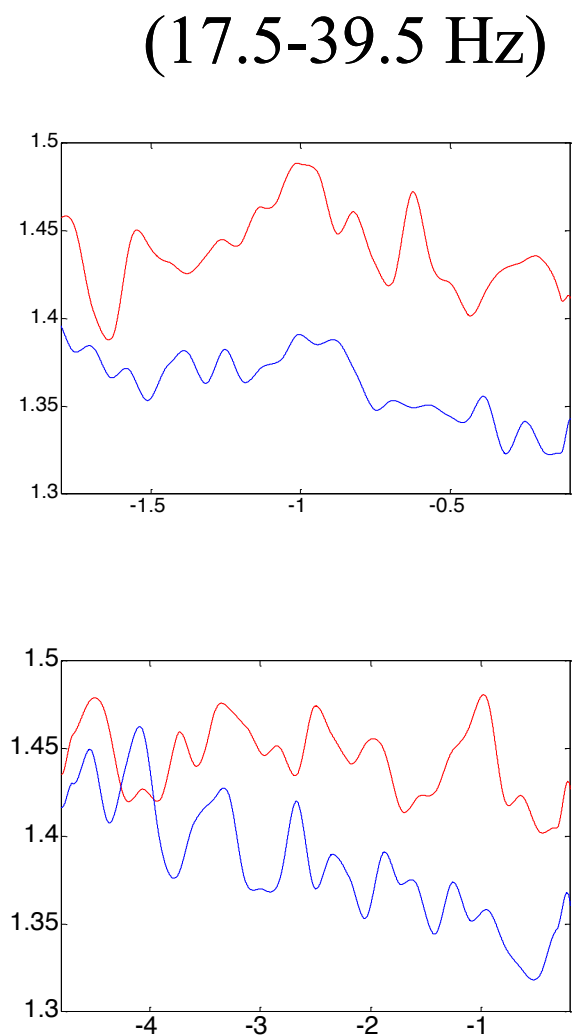

Time relative to stimulus ( $\mathrm{sec}$ )

Figure 32. The time course of pre-TMS power difference. The EEG recorded at C3 electrode was filtered in alpha (6.5-11.5 Hz) and beta (17.5-39.5 Hz) bands. The RMS of the filtered EEG was computed over trials, smoothed using local regression and grand averaged. The upper row shows the data from 2 sec preceding the TMS pulse and the lower row - data from a different subset of trials where 5 sec preceding the movement could be analyzed. The red curve corresponds to the high MEP amplitude trials and the blue curve to the low MEP amplitude trials. It can be seen that the effects starts at least 4 sec before the stimulus. 


\subsubsection{Comparison of scalp current density (SCD) maps between pre-TMS and movement data}

Figure 33 shows the SCD maps of power differences between high and low MEP amplitude trials (Figure 33A) and time period around movement onset vs. baseline (Figure 33B). The maps are similar between the two cases in both alpha and beta ranges. However the maximal differences on the left side in the case of movement seem to be slightly more posterior to the maximal differences in pre-TMS data. In both cases the maximal difference is seen at electrode C3. In the pre-TMS data a large difference is also observed at FC1, whereas in movement data at CP3 and CP1.

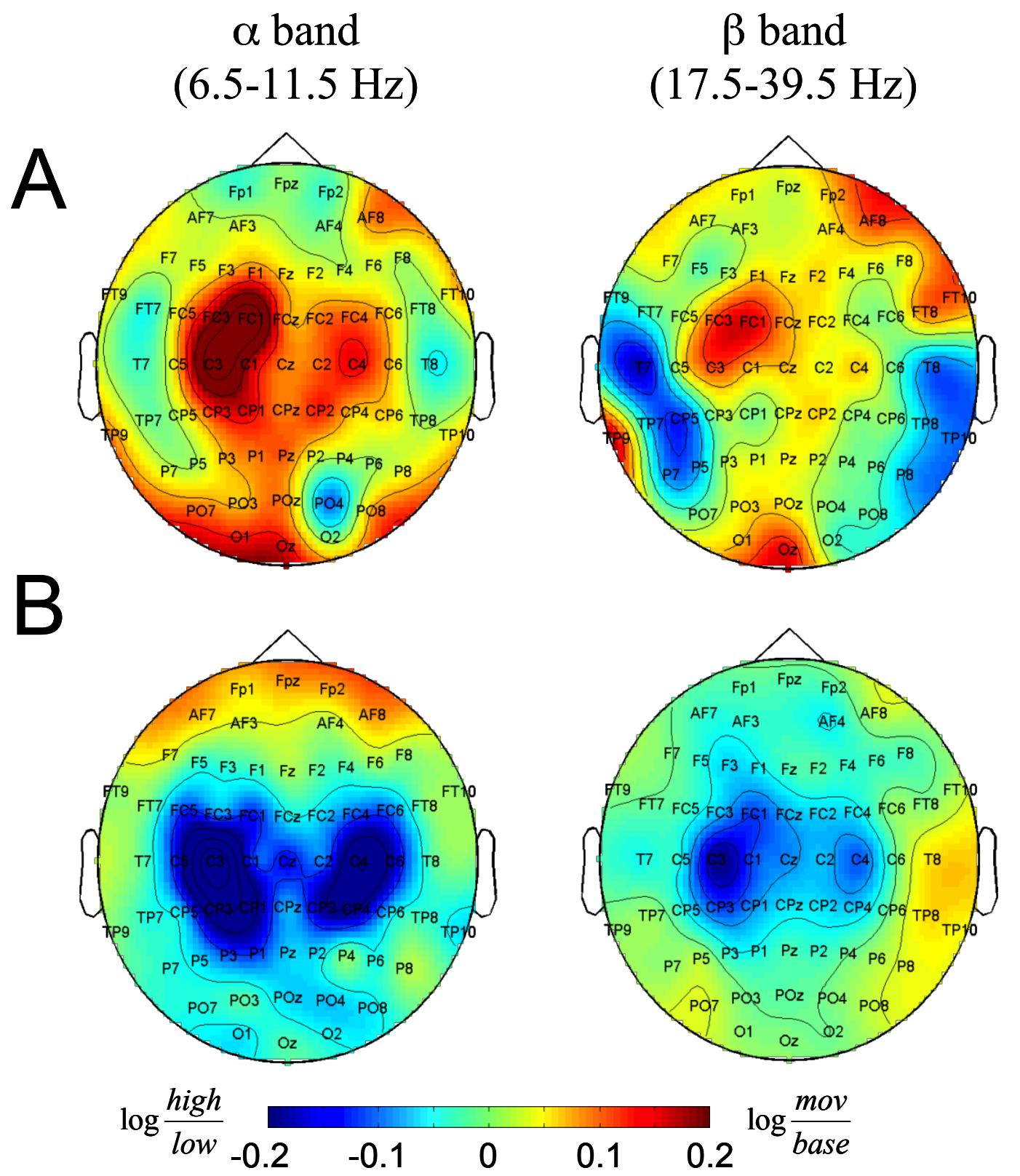

Figure 33. Scalp current density difference patterns for the pre-TMS data (A) and movement data (B). SCD was computed prior to spectral analysis. Note the similarity between the two cases with the slight forward shift in (A). 


\subsubsection{Correlation between the pre-TMS power difference and ERD}

To further clarify the question of whether the same physiological processes are involved in MEP amplitude modulation and movement execution, the correlation between the two effects was examined. The correlation was computed over subjects between the differences of log power at channel C3 for pre-TMS data (Figure 29B) and movement data (Figure 31B), separately for alpha $(6.5-11.5 \mathrm{~Hz})$ and beta (17.5$39.5 \mathrm{~Hz}$ ) range. The results are shown in Figure 34. In both cases a significant correlation was found $(\mathrm{p}<0.05)$.
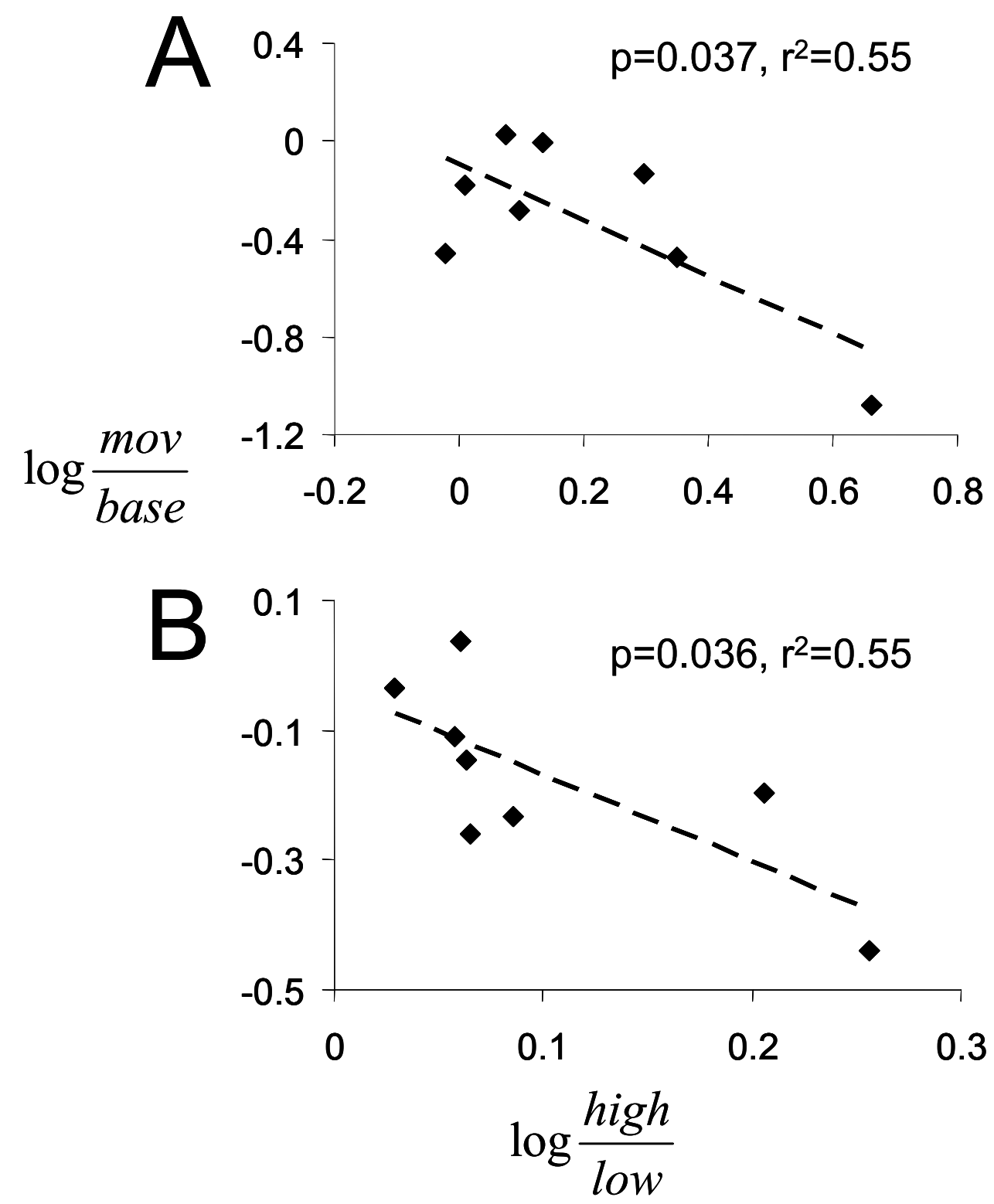

Figure 34. Correlation across subjects between the pre-TMS and ERD effects. The correlation was computed between $\log$ power differences at the $\mathrm{C} 3$ electrode for the alpha band (A) and beta band (B). In both cases a significant correlation was found. 


\subsubsection{Discussion}

The present study addresses two important questions that arose in the recent years in the study of the motor system - the cause of high variability of the amplitude of motor evoked potentials and the role of oscillations generated in the sensorimotor loop. We show that the two phenomena are related. This result emerged from a data-driven nonparametric statistical analysis with minimal prior assumptions.

The main novel finding of the present study is that increased MEP amplitude is associated with increased power of EEG oscillations recorded over the sensorimotor cortex during at least $2 \mathrm{sec}$ prior to a TMS pulse. The increase is observed in two distinct frequency bands 6.5 to $11.5 \mathrm{~Hz}$ and 17.5 to $39.5 \mathrm{~Hz}$ that overlap the traditionally defined alpha and beta range, respectively. The direction of the effect is unexpected since, despite contradicting findings on the relation between oscillations and cortical excitability in general (Ploner et al., 2006), in the motor system increased oscillatory activity has been associated with an idling state and a decrease with movement planning and execution (Neuper and Pfurtscheller, 2001). Moreover, studies where magnetic stimulation was at different phases of movement showed that MEP amplitude increases around the onset of EMG activity around the same time when ERD is usually observed (Rau et al., 2003; Zaaroor et al., 2003).

The principal difference between those studies and the present study is that in the present study the subjects were instructed to maintain constant relaxation and did not perform any task. Thus, no movements took place and the differences between the different stimuli originate in spontaneous fluctuations in the brain's functional state. In this case the EEG correlate of increased motor excitability might be different. Moreover, our results imply that the notion of 'excitability' as a single well defined parameter that can be measured at any given instant, is positively correlated with MEP amplitude and negatively with the power of EEG oscillations in the alpha and beta range is over-simplified. Rather, the sensorimotor loop can work in a number of modes or states each characterized by a different combination of physiological parameters.

Oscillations in the beta range, for instance, have been linked to idling state (Niedermeyer, 1999) and active inhibition of motor performance (Pfurtscheller et al., 
2003) and shown to increase in amplitude in response to increase of cortical inhibition (Jensen et al., 2005), but were also found to be associated with active exploration (Murthy and Fetz, 1992) movement planning (Donoghue et al., 1998) (Sanes and Donoghue, 1993) and attention or arousal (Fetz et al., 2000), with no relation between the oscillations and specific movement parameters (Donoghue et al., 1998). While in the former case one would expect beta oscillations to be associated with decreased MEP amplitude, in the latter case the opposite would quite likely be true.

It has been shown (Paus et al., 2001a; Van Der Werf and Paus, 2006) that motor cortex TMS induces a period of synchronized activity in the beta range in the vicinity of the stimulation site. These oscillations can be interpreted as transient synchronization of spontaneous activity within the beta band, or as activation of idling neurons that begin to oscillate as a consequence of stimulation. In both cases the finding of stronger beta oscillations prior to the stimulus may increase the effect of TMS on the cortex and the stronger effect on the cortex might be associated with a stronger effect on the target muscle.

Another explanation for the surprising direction of the effect in our study is the suggestion that beta oscillations are correlated with maintenance of posture and preservation of the present motor set, and this maintenance being an active process rather than just passive inhibition of movement (Gilbertson et al., 2005). In this case beta oscillations and higher motor excitability may be two components of the process aimed at resisting changes in posture.

\subsubsection{Possible sources}

Comparison of the scalp maps of power differences between the pre-TMS and movement-associated ERD may provide information about cortical generators correlated with MEP amplitude and their relation to structures associated with movement. In the present study even small differences in the scalp maps might be meaningful since both magnetic stimulation and self-paced movement were performed during the same experimental session with exactly the same electrode positions. Comparing the scalp current density patterns which are especially sensitive to source location (Figure 33) reveals quite remarkable similarity in both alpha and beta bands between the two cases. It is especially remarkable given the fact the pre- 
TMS pattern (Figure 33A) was extracted from EEG recorded while the subject was not performing any task or reacting to a stimulus and there was nothing in the analysis procedure looking for effects in the sensorimotor area. Nevertheless, there was a small forward shift in the pre-TMS effect relative to ERD. This might be related to the fact that performing a movement includes processing sensory feedback involving S1 while changes in MEP amplitude are more specific to motor phenomenon.

The frequency ranges in which the pre-TMS effect was observed are very similar to the frequency range of the ERD. In the case of pre-TMS the effect is more clearly separated into two separate sub-ranges in the alpha and beta bands. As in the case of the spatial pattern, it is remarkable that the pattern in frequency is consistent with the known properties of alpha and beta rhythms (Neuper and Pfurtscheller, 2001) that emerge in data-driven analysis of EEG.

The correlation across subjects between the magnitude of ERD and the pre-TMS effect is a further indication of the functional link between the two phenomena, although the reasons for the existence of this correlation might be purely anatomical.

\subsubsection{Time scale of MEP changes correlated with EEG power}

Close examination of Figure 28 shows that apart from rapid and seemingly random variation in MEP amplitude between neighboring trials, there is also slower variation in the average amplitude over the course of the entire recording session. This variation is likely to reflect changes in the subject's overall vigilance and attention shifts to and from the stimulated hand (Rossini et al., 1999). In our sorting of trials by their MEP amplitude we did not distinguish between the two processes. Thus our analysis cannot rule out the possibility that the changes in the scalp pattern we revealed reflect the slow attentional change rather than the fast trial-to-trial change in brain excitability. Analysis shown in Figure 32 demonstrates that the differences in EEG power between high- and low- amplitude MEP trials precede the magnetic stimulus by at least $4 \mathrm{sec}$. Perhaps the actual duration of the effect is much longer but it cannot be shown with the stimulation rate we used. Moreover, the changes in ongoing cortical activity are unlikely to be the only source of variance in the MEP amplitude and attempts we made to predict the MEP amplitude on a single trial basis based on the EEG showed that although a significant correlation can be demonstrated for some subjects, the 
percentage of variance explained is small (data not shown). This, however, is not surprising since estimation of power on a single trial basis is highly unreliable and there is substantial intrinsic variance just in the estimation process.

\subsubsection{Novel way of examining ongoing activity}

Ongoing brain activity has long been considered an important factor in shaping behavior. The majority of cortical neurons project to other cortical neurons and the input and output comprise only a small fraction of information processed in the cortex (Abeles, 1991). However, it is very difficult to study behaviorally relevant ongoing activity in humans due to the absence of a proper non-invasive methodology. The problem is especially severe for MEG and EEG that usually require averaging of a large number of trials around specific external events. This averaging eliminates ongoing activity that is not time locked to the event. The technique used in the present study makes it possible to reveal the sources of ongoing activity taking place in the absence of any specific task based on an event occurring later in time. While some work has been done to study the EEG responses following a magnetic stimulus (Komssi and Kahkonen, 2006) the present study offers a new way to explore the EEG activity preceding TMS in a way not previously attempted. This technique is advantageous from the technical point of view since EEG preceding the TMS pulse is not contaminated by TMS artifacts (Virtanen et al., 1999) and with careful choice of digital filters can be studied with no special TMS-adapted hardware and software tools. Our study was based on analyzing motor evoked potentials which are specific to the motor system. However, the method can be extended to analyzing the pre-stimulus EEG activity based on the post-stimulus EEG response. This might be possible since at least in some cases TMS evokes responses large enough to be seen in single trials (Bender et al., 2005). This research direction might lead to the development of a method to study the ongoing activity in a wide range of cortical regions.

\subsubsection{Conclusions}

The present study showed for the first time that the power of EEG oscillations in the time period preceding the TMS pulse is positively correlated with the MEP amplitude. The pattern of power differences revealed based on purely data-driven analysis was remarkably similar to ERD pattern associated with movement planning and 
performance and matched the known properties of oscillations in the alpha and beta ranges generated by the sensorimotor loop. The findings demonstrate the existence and physiological significance of spontaneous modulations in oscillatory cortical activity in the absence of a task. 


\section{Discussion and Conclusions}

In this section we would like to go beyond the study specific details that have already been discussed in each of the respective chapters and provide a general overview of the significance of the presented studies and the ways their results can be integrated and extended.

We started the project with the idea of developing a closes loop system for modification of cortical functional states and connectivity. The main achievement of our studies is the development of tools for better characterization of the short- and long-term effects of TMS on the human brain. These tools may one day serve as a part of such a closed loop system.

In the study of EEG responses to TMS we were able to substantially reduce the artifact contamination of the early part of the TMS evoked ERP and isolate distinct contributions of several brain regions to the response. These contributions had distinctive time courses, making it possible to determine the time of activation of the corresponding brain areas. In our study we focused on analyzing the features of the response common to all subjects, features that survived the grand average computation. This was one of the limitations of analyzing data contributed by another lab where the number of trials recorded for each subject was relatively low. However, even from this data it was clear that there is remarkable inter-subject variability in the responses. Further studies are necessary in order to verify that this variability originates in the variable functional brain connectivity rather than in subtle differences in the experimental procedures. Nevertheless, it would be reasonable to assume that at least some of the inter-subject differences have physiological basis. The recent TMS studies of the changes of functional connectivity during sleep (Massimini et al., 2005a) and the differences between healthy subjects and patients suffering from schizophrenia (Massimini et al., 2005b) imply that functional connectivity seen with TMS can vary both as part of normal physiological processes and as a result of disease. The methodology developed for this study makes it possible to analyze the resulting differences in a detailed way. Until now only few stimulation sites have been explored. One of the reasons for this was the necessity to select stimulation sites in a way that would reduce the TMS artifact in the EEG. Availability of artifact removal methodology has the potential to lift this limitation and pave the 
way to systematic exploration of responses to TMS delivered at all cortical sites accessible to magnetic stimulation and building functional connectivity maps at the level of cortical areas. This analysis can be one of the methods for building the 'human brain connectome' (a recently suggested term analogous to 'genome' (Sporns et al., 2005)). It may also lead in the future to development of novel diagnostic tools for neurology and psychiatry.

The paired associative stimulation protocol is a tool for precise modification of cortical connectivity exploiting the known precise relations between different neural events. Our study showed that the effect of PAS can be very specific at both regional and laminar level and its effect can be modulated in a purposeful way by changing the stimulation parameters. Multichannel EEG combined with sophisticated analysis methods can be used to characterize the PAS effect and thereby improve its specificity. We showed that 64-channel EEG can resolve at least 3 distinct processes in the sensorimotor cortical areas that are associated with electrical stimulation of the median nerve: the activation of area $3 \mathrm{~b}$, the activation of area 1 and the proprioceptive input processing possibly in area $3 \mathrm{a}$. Two of these processes - could be specifically modified by PAS. The third process - activation of area 1 was not affected for the PAS conditions we tested. It would be interesting to see whether PAS interval can be found specifically targeting area 1. A positive answer would indicate that TMS effects can be focused in space beyond the spatial resolution of the TMS device itself by exploiting the response properties of different areas.

For the reasons of simplicity and convenience our study of spike timing dependent plasticity in the human cortex was based on pairing TMS with a sensory stimulus. However, this approach is limited to primary sensory areas or areas strongly connected to them (such as M1). A more general approach would be to perform paired stimulation with two TMS coils activating two cortical areas in order to modify the strength of the connections between these areas. To make this possible information about functional connections between cortical areas and about propagation delays of these connections is crucial. As we showed, even very small differences in interstimulus interval can greatly affect the outcome of a PAS intervention. Exactly this kind of information can be provided by the TMS based functional connectivity mapping. Thus combining the methodology for 
characterization of TMS evoked responses with the PAS methodology would be a very promising research direction.

Until now we have discussed initiation of neural events using TMS or combining TMS stimulation with neural events initiated by sensory stimuli. However, for many clinically useful applications TMS should be applied in response to some changes in spontaneous brain activity. An analogous mechanism can be seen in the present artificial cardiac pacemakers that are only activated in response to abnormal heart activity patterns. The MEP variability study shows a possible direction for characterization of the ongoing activity in the human brain. The novel TMS based methodology developed for this study may be used to reveal spontaneously active cortical sources interacting with the area of interest. The particular methodology of this study is limited to the motor system since it uses a contrast based on MEP amplitude and MEP is a motor phenomenon. However, further development of the methodology for characterization of TMS evoked ERPs may lead to analyzing responses distinguishable in single trials. Such large responses have already been described in children (Bender et al., 2005). Combining the methodologies of both studies it might be possible to reveal ongoing activity sources in the EEG segments preceding the TMS pulse using contrasts based on the EEG responses occurring after the TMS pulse. This methodology would be a complementary method for functional connectivity mapping, in a way more natural than studying TMS evoked ERPs, because it would reveal brain areas affecting the simulated site's excitability during the brain's natural activity.

An additional important contribution of our studies was one of the first applications of the novel nonparametric statistical tools for EEG analysis. At the time when our project was at its initial stages one of the serious challenges we envisioned was the problem of extracting meaningful information from the huge amounts of multidimensional EEG data. This problem was especially severe in our case in comparison with other ERP studies because the literature about EEG responses to TMS amounted to just a few papers then and it was not clear where to look for effects and how to characterize them. The nonparametric statistical methodology combined with source analysis provided a very good solution for this difficult problem that at times seemed completely intractable. The study of PAS effects was an excellent test case where this methodology was applied for the first time. The initial problem in that 
study was distinguishing between two very clearly stated hypotheses - attribution of PAS effect at a specific time window to either tangential or radial source. The simplicity of the problem made it possible for us to understand very well the statistical methodology used to tackle it. With this understanding we could use similar methodology to reveal two unexpected effects - the effect of PASN20+100ms on a distinct possibly proprioceptive cortical area and the relation between MN-SSEP changes and the changes in two-point discrimination threshold following PASN20$2.5 \mathrm{~ms}$. This led to even more remarkable result presented in the last study. An effect whose signature in both space and frequency indicate its likely physiological origin was revealed in spontaneous EEG data without any prior expectations. Since the direction of the effect is at first glance counter-intuitive it would be very likely to be missed if a more restrictive approach was used.

In summary, integration and further extension of the methodologies presented above can lead to development of very useful clinical and research tools. It is certainly our hope that these promising research directions will be followed in the future. 


\section{References}

Abeles M (1991) Corticonics : neural circuits of the cerebral cortex. Cambridge ; New York: Cambridge University Press.

Allen CB, Celikel T, Feldman DE (2003) Long-term depression induced by sensory deprivation during cortical map plasticity in vivo. Nat Neurosci 6:291-299.

Allison T, McCarthy G, Wood CC, Jones SJ (1991) Potentials evoked in human and monkey cerebral cortex by stimulation of the median nerve. A review of scalp and intracranial recordings. Brain 114:2465-2503.

Amassian VE, Quirk GJ, Stewart M (1990) A comparison of corticospinal activation by magnetic coil and electrical stimulation of monkey motor cortex. Electroencephalogr Clin Neurophysiol 77:390-401.

Amedi A, Malach R, Pascual-Leone A (2005) Negative BOLD differentiates visual imagery and perception. Neuron 48:859-872.

Andersen P, Sundberg SH, Sveen O, Wigstrom H (1977) Specific long-lasting potentiation of synaptic transmission in hippocampal slices. Nature 266:736737.

Antal A, Nitsche MA, Kincses TZ, Lampe C, Paulus W (2004) No correlation between moving phosphene and motor thresholds: a transcranial magnetic stimulation study. Neuroreport 15:297-302.

Arieli A, Sterkin A, Grinvald A, Aertsen A (1996) Dynamics of ongoing activity: explanation of the large variability in evoked cortical responses. Science 273:1868-1871.

Awiszus F (2003) TMS and threshold hunting. Suppl Clin Neurophysiol 56:13-23.

Balzamo E, Marquis P, Chauvel P, Regis J (2004) Short-latency components of evoked potentials to median nerve stimulation recorded by intracerebral electrodes in the human pre- and postcentral areas. Clin Neurophysiol 115:1616-1623.

Barker AT, Jalinous R, Freeston IL (1985) Non-invasive magnetic stimulation of human motor cortex. Lancet 1:1106-1107.

Barnes CA, Jung MW, McNaughton BL, Korol DL, Andreasson K, Worley PF (1994) LTP saturation and spatial learning disruption: effects of task variables and saturation levels. J Neurosci 14:5793-5806.

Bast T, Ramantani G, Boppel T, Metzke T, Ozkan O, Stippich C, Seitz A, Rupp A, Rating D, Scherg M (2005) Source analysis of interictal spikes in 
polymicrogyria: loss of relevant cortical fissures requires simultaneous EEG to avoid MEG misinterpretation. Neuroimage 25:1232-1241.

Baudewig J, Siebner HR, Bestmann S, Tergau F, Tings T, Paulus W, Frahm J (2001) Functional MRI of cortical activations induced by transcranial magnetic stimulation (TMS). Neuroreport 12:3543-3548.

Baumer T, Bock F, Koch G, Lange R, Rothwell JC, Siebner HR, Munchau A (2006) Magnetic stimulation of human premotor or motor cortex produces interhemispheric facilitation through distinct pathways. J Physiol 572:857868.

Bendat JS, Piersol AG (2000) Random data : analysis and measurement procedures, 3rd Edition. New York: Wiley.

Bender S, Basseler K, Sebastian I, Resch F, Kammer T, Oelkers-Ax R, Weisbrod M (2005) Electroencephalographic response to transcranial magnetic stimulation in children: Evidence for giant inhibitory potentials. Ann Neurol 58:58-67.

Berg P, Scherg M (1994a) A multiple source approach to the correction of eye artifacts. Electroencephalogr Clin Neurophysiol 90:229-241.

Berg P, Scherg M (1994b) A fast method for forward computation of multiple-shell spherical head models. Electroencephalogr Clin Neurophysiol 90:58-64.

Bestmann S, Baudewig J, Siebner HR, Rothwell JC, Frahm J (2003) Subthreshold high-frequency TMS of human primary motor cortex modulates interconnected frontal motor areas as detected by interleaved fMRI-TMS. Neuroimage 20:1685-1696.

Bestmann S, Baudewig J, Siebner HR, Rothwell JC, Frahm J (2004) Functional MRI of the immediate impact of transcranial magnetic stimulation on cortical and subcortical motor circuits. Eur J Neurosci 19:1950-1962.

Bestmann S, Baudewig J, Siebner HR, Rothwell JC, Frahm J (2005) BOLD MRI responses to repetitive TMS over human dorsal premotor cortex. Neuroimage 28:22-29.

Bliss TV, Lomo T (1973) Long-lasting potentiation of synaptic transmission in the dentate area of the anaesthetized rabbit following stimulation of the perforant path. J Physiol 232:331-356.

Bliss TV, Gardner-Medwin AR (1973) Long-lasting potentiation of synaptic transmission in the dentate area of the unanaestetized rabbit following stimulation of the perforant path. J Physiol 232:357-374. 
Bohning DE, Shastri A, Wassermann EM, Ziemann U, Lorberbaum JP, Nahas Z, Lomarev MP, George MS (2000) BOLD-f MRI response to single-pulse transcranial magnetic stimulation (TMS). J Magn Reson Imaging 11:569-574.

Bohning DE, Shastri A, McConnell KA, Nahas Z, Lorberbaum JP, Roberts DR, Teneback C, Vincent DJ, George MS (1999) A combined TMS/fMRI study of intensity-dependent TMS over motor cortex. Biol Psychiatry 45:385-394.

Bonato C, Miniussi C, Rossini PM (2006) Transcranial magnetic stimulation and cortical evoked potentials: A TMS/EEG co-registration study. Clin Neurophysiol.

Boutros NN, Berman RM, Hoffman R, Miano AP, Campbell D, Ilmoniemi R (2000) Electroencephalogram and repetitive transcranial magnetic stimulation. Depress Anxiety 12:166-169.

Brasil-Neto JP, McShane LM, Fuhr P, Hallett M, Cohen LG (1992) Topographic mapping of the human motor cortex with magnetic stimulation: factors affecting accuracy and reproducibility. Electroencephalogr Clin Neurophysiol 85:9-16.

Brown H, Prescott R (2006) Applied mixed models in medicine, 2nd Edition. Chichester ; Hoboken, NJ: Wiley.

Brown P, Williams D (2005) Basal ganglia local field potential activity: character and functional significance in the human. Clin Neurophysiol 116:2510-2519.

Buchner H, Adams L, Muller A, Ludwig I, Knepper A, Thron A, Niemann K, Scherg M (1995) Somatotopy of human hand somatosensory cortex revealed by dipole source analysis of early somatosensory evoked potentials and 3D-NMR tomography. Electroencephalogr Clin Neurophysiol 96:121-134.

Buchner H, Adams L, Knepper A, Ruger R, Laborde G, Gilsbach JM, Ludwig I, Reul J, Scherg M (1994) Preoperative localization of the central sulcus by dipole source analysis of early somatosensory evoked potentials and threedimensional magnetic resonance imaging. J Neurosurg 80:849-856.

Carandini M, Heeger DJ, Senn W (2002) A synaptic explanation of suppression in visual cortex. J Neurosci 22:10053-10065.

Celikel T, Szostak VA, Feldman DE (2004) Modulation of spike timing by sensory deprivation during induction of cortical map plasticity. Nat Neurosci 7:534541. 
Cerri G, Shimazu H, Maier MA, Lemon RN (2003) Facilitation from ventral premotor cortex of primary motor cortex outputs to macaque hand muscles. J Neurophysiol 90:832-842.

Challiner V, Griffiths L (2000) Electroconvulsive therapy: a review of the literature. J Psychiatr Ment Health Nurs 7:191-198.

Chatrian GE, Lettich E, Nelson PL (1988) Modified nomenclature for the "10\%" electrode system. J Clin Neurophysiol 5:183-186.

Chen R, Classen J, Gerloff C, Celnik P, Wassermann EM, Hallett M, Cohen LG (1997) Depression of motor cortex excitability by low-frequency transcranial magnetic stimulation. Neurology 48:1398-1403.

Civardi C, Cantello R, Asselman P, Rothwell JC (2001) Transcranial magnetic stimulation can be used to test connections to primary motor areas from frontal and medial cortex in humans. Neuroimage 14:1444-1453.

Cohen LG, Ziemann U, Chen R, Classen J, Hallett M, Gerloff C, Butefisch C (1998) Studies of neuroplasticity with transcranial magnetic stimulation. J Clin Neurophysiol 15:305-324.

Cooke SF, Bliss TV (2006) Plasticity in the human central nervous system. Brain.

Cooper R, Osselton JW, Shaw JC (1980) EEG technology, 3d Edition. London ; Boston: Butterworths.

Corthout E, Uttl B, Juan CH, Hallett M, Cowey A (2000) Suppression of vision by transcranial magnetic stimulation: a third mechanism. Neuroreport 11:23452349.

Cowey A, Walsh V (2001) Tickling the brain: studying visual sensation, perception and cognition by transcranial magnetic stimulation. Prog Brain Res 134:411425.

Dan Y, Poo M-M (2006) Spike Timing-Dependent Plasticity: From Synapse to Perception. Physiol Rev 86:1033-1048.

Darian-Smith C, Darian-Smith I, Burman K, Ratcliffe N (1993) Ipsilateral cortical projections to areas $3 \mathrm{a}, 3 \mathrm{~b}$, and 4 in the macaque monkey. J Comp Neurol 335:200-213.

Darling WG, Wolf SL, Butler AJ (2006) Variability of motor potentials evoked by transcranial magnetic stimulation depends on muscle activation. Exp Brain Res. 
Daskalakis ZJ, Paradiso GO, Christensen BK, Fitzgerald PB, Gunraj C, Chen R (2004) Exploring the connectivity between the cerebellum and motor cortex in humans. J Physiol 557:689-700.

David O, Kilner JM, Friston KJ (2006) Mechanisms of evoked and induced responses in MEG/EEG. Neuroimage.

Defebvre L, Bourriez JL, Dujardin K, Derambure P, Destee A, Guieu JD (1994) Spatiotemporal study of Bereitschaftspotential and event-related desynchronization during voluntary movement in Parkinson's disease. Brain Topogr 6:237-244.

Denslow S, Lomarev M, George MS, Bohning DE (2005) Cortical and subcortical brain effects of transcranial magnetic stimulation (TMS)-induced movement: an interleaved TMS/functional magnetic resonance imaging study. Biol Psychiatry 57:752-760.

Devanne H, Cohen LG, Kouchtir-Devanne N, Capaday C (2002) Integrated motor cortical control of task-related muscles during pointing in humans. J Neurophysiol 87:3006-3017.

Di Russo F, Martinez A, Sereno MI, Pitzalis S, Hillyard SA (2002) Cortical sources of the early components of the visual evoked potential. Hum Brain Mapp 15:95-111.

Dinse HR, Ragert P, Pleger B, Schwenkreis P, Tegenthoff M (2003) Pharmacological modulation of perceptual learning and associated cortical reorganization. Science 301:91-94.

Donoghue JP, Sanes JN, Hatsopoulos NG, Gaal G (1998) Neural discharge and local field potential oscillations in primate motor cortex during voluntary movements. J Neurophysiol 79:159-173.

Doyle LM, Kuhn AA, Hariz M, Kupsch A, Schneider GH, Brown P (2005) Levodopa-induced modulation of subthalamic beta oscillations during selfpaced movements in patients with Parkinson's disease. Eur J Neurosci 21:1403-1412.

Druschky K, Kaltenhauser M, Hummel C, Druschky A, Huk WJ, Neundorfer B, Stefan H (2003) Somatosensory evoked magnetic fields following passive movement compared with tactile stimulation of the index finger. Exp Brain Res 148:186-195. 
Dunwiddie T, Lynch G (1978) Long-term potentiation and depression of synaptic responses in the rat hippocampus: localization and frequency dependency. $\mathrm{J}$ Physiol 276:353-367.

Elbert T, Lutzenberger W, Rockstroh B, Birbaumer N (1981a) The influence of lowlevel transcortical DC-currents on response speed in humans. Int J Neurosci 14:101-114.

Elbert T, Rockstroh B, Lutzenberger W, Birbaumer N (1981b) The influence of lowlevel, event-related DC-currents during time estimation in humans. Int J Neurosci 15:103-106.

Epstein CM (1999) Language and TMS/rTMS. Electroencephalogr Clin Neurophysiol Suppl 51:325-333.

Esser SK, Huber R, Massimini M, Peterson MJ, Ferrarelli F, Tononi G (2006) A direct demonstration of cortical LTP in humans: A combined TMS/EEG study. Brain Res Bull 69:86-94.

Feldman DE, Brecht M (2005) Map plasticity in somatosensory cortex. Science 310:810-815.

Fell J, Dietl T, Grunwald T, Kurthen M, Klaver P, Trautner P, Schaller C, Elger CE, Fernandez G (2004) Neural bases of cognitive ERPs: more than phase reset. J Cogn Neurosci 16:1595-1604.

Fetz EE, Chen D, Murthy VN, Matsumura M (2000) Synaptic interactions mediating synchrony and oscillations in primate sensorimotor cortex. J Physiol Paris 94:323-331.

Fox P, Ingham R, George MS, Mayberg H, Ingham J, Roby J, Martin C, Jerabek P (1997) Imaging human intra-cerebral connectivity by PET during TMS. Neuroreport 8:2787-2791.

Fox PT, Narayana S, Tandon N, Fox SP, Sandoval H, Kochunov P, Capaday C, Lancaster JL (2005) Intensity modulation of TMS-induced cortical excitation: Primary motor cortex. Hum Brain Mapp.

Froc DJ, Racine RJ (2005) Interactions between LTP- and LTD-inducing stimulation in the sensorimotor cortex of the awake freely moving rat. J Neurophysiol 93:548-556.

Froc DJ, Chapman CA, Trepel C, Racine RJ (2000) Long-term depression and depotentiation in the sensorimotor cortex of the freely moving rat. $\mathrm{J}$ Neurosci 20:438-445. 
Froemke RC, Poo MM, Dan Y (2005) Spike-timing-dependent synaptic plasticity depends on dendritic location. Nature 434:221-225.

Fu YX, Djupsund K, Gao H, Hayden B, Shen K, Dan Y (2002) Temporal specificity in the cortical plasticity of visual space representation. Science 296:19992003.

Fuggetta G, Fiaschi A, Manganotti P (2005) Modulation of cortical oscillatory activities induced by varying single-pulse transcranial magnetic stimulation intensity over the left primary motor area: a combined EEG and TMS study. Neuroimage 27:896-908.

Fuggetta G, Pavone EF, Walsh V, Kiss M, Eimer M (2006) Cortico-cortical interactions in spatial attention: A combined ERP/TMS study. J Neurophysiol.

Garcia Larrea L, Bastuji H, Mauguiere F (1992) Unmasking of cortical SEP components by changes in stimulus rate: a topographic study. Electroencephalogr Clin Neurophysiol 84:71-83.

George MS (2003) Stimulating the Brain. Scientific American

Scientific American J1 - Scientific American 289:66.

Gerloff C, Braun C, Staudt M, Hegner YL, Dichgans J, Krageloh-Mann I (2006) Coherent corticomuscular oscillations originate from primary motor cortex: Evidence from patients with early brain lesions. Hum Brain Mapp.

Gevins A, Remond A, eds (1987) Methods of Analysis of Brain Electrical and Magnetic Signals. Amsterdam-New York - Oxford: Elseiver.

Geyer S, Schleicher A, Schormann T, Mohlberg H, Bodegard A, Roland PE, Zilles K (2001) Integration of microstructural and functional aspects of human somatosensory areas $3 \mathrm{a}, 3 \mathrm{~b}$, and 1 on the basis of a computerized brain atlas. Anat Embryol (Berl) 204:351-366.

Gilbertson T, Lalo E, Doyle L, Di Lazzaro V, Cioni B, Brown P (2005) Existing motor state is favored at the expense of new movement during 13-35 Hz oscillatory synchrony in the human corticospinal system. J Neurosci 25:77717779 .

Gross J, Kujala J, Hamalainen M, Timmermann L, Schnitzler A, Salmelin R (2001) Dynamic imaging of coherent sources: Studying neural interactions in the human brain. Proc Natl Acad Sci U S A 98:694-699.

Gross J, Timmermann L, Kujala J, Dirks M, Schmitz F, Salmelin R, Schnitzler A (2002) The neural basis of intermittent motor control in humans. Proc Natl Acad Sci U S A 99:2299-2302. 
Haaland KY, Harrington DL (1996) Hemispheric asymmetry of movement. Curr Opin Neurobiol 6:796-800.

Hallett M (2000) Transcranial magnetic stimulation and the human brain. Nature 406:147-150.

Hamalainen MS, Ilmoniemi RJ (1994) Interpreting magnetic fields of the brain: minimum norm estimates. Med Biol Eng Comput 32:35-42.

Heller L, van Hulsteyn DB (1992) Brain stimulation using electromagnetic sources: theoretical aspects. Biophys J 63:129-138.

Herwig U, Satrapi P, Schonfeldt-Lecuona C (2003) Using the international 10-20 EEG system for positioning of transcranial magnetic stimulation. Brain Topogr 16:95-99.

Hjorth B (1991) Principles for transformation of scalp EEG from potential field into source distribution. J Clin Neurophysiol 8:391-396.

Hodzic A, Veit R, Karim AA, Erb M, Godde B (2004) Improvement and decline in tactile discrimination behavior after cortical plasticity induced by passive tactile coactivation. J Neurosci 24:442-446.

Hoechstetter K, Bornfleth H, Weckesser D, Ille N, Berg P, Scherg M (2004) BESA source coherence: a new method to study cortical oscillatory coupling. Brain Topogr 16:233-238.

Huang MX, Aine C, Davis L, Butman J, Christner R, Weisend M, Stephen J, Meyer J, Silveri J, Herman M, Lee RR (2000) Sources on the anterior and posterior banks of the central sulcus identified from magnetic somatosensory evoked responses using multistart spatio-temporal localization. Hum Brain Mapp 11:59-76.

Huang YZ, Edwards MJ, Rounis E, Bhatia KP, Rothwell JC (2005) Theta burst stimulation of the human motor cortex. Neuron 45:201-206.

Ille N, Berg P, Scherg M (2002) Artifact correction of the ongoing EEG using spatial filters based on artifact and brain signal topographies. J Clin Neurophysiol 19:113-124.

Ilmoniemi RJ, Ruohonen J, Virtanen J, Aronen HJ, Karhu J (1999) EEG responses evoked by transcranial magnetic stimulation. Electroencephalogr Clin Neurophysiol Suppl 51:22-29.

Ilmoniemi RJ, Virtanen J, Ruohonen J, Karhu J, Aronen HJ, Naatanen R, Katila T (1997) Neuronal responses to magnetic stimulation reveal cortical reactivity and connectivity. Neuroreport 8:3537-3540. 
Izumi S, Findley TW, Ikai T, Andrews J, Daum M, Chino N (1995) Facilitatory effect of thinking about movement on motor-evoked potentials to transcranial magnetic stimulation of the brain. Am J Phys Med Rehabil 74:207-213.

Jalinous R (1998) A guide to magnetic stimulation. In: www.magstim.com.

Jasper H (1958) The ten-twenty electrode system of the International Federation. Electroencephalogr Clin Neurophysiol 10:371-375.

Jensen O, Goel P, Kopell N, Pohja M, Hari R, Ermentrout B (2005) On the human sensorimotor-cortex beta rhythm: sources and modeling. Neuroimage 26:347355.

Jing H, Takigawa M (2000) Observation of EEG coherence after repetitive transcranial magnetic stimulation. Clin Neurophysiol 111:1620-1631.

Jing H, Takigawa M, Okamura H, Doi W, Fukuzako H (2001a) Comparisons of event-related potentials after repetitive transcranial magnetic stimulation. J Neurol 248:184-192.

Jing H, Takigawa M, Hamada K, Okamura H, Kawaika Y, Yonezawa T, Fukuzako H (2001b) Effects of high frequency repetitive transcranial magnetic stimulation on P(300) event-related potentials. Clin Neurophysiol 112:304-313.

Jones E (1986) Connectivity of the primate sensory-motor cortex. In: Cerebral cortex (Jones E, Peters A, eds), pp 113-183. New York: Plenum.

Kahkonen S, Wilenius J, Komssi S, Ilmoniemi RJ (2004) Distinct differences in cortical reactivity of motor and prefrontal cortices to magnetic stimulation. Clin Neurophysiol 115:583-588.

Kahkonen S, Komssi S, Wilenius J, Ilmoniemi RJ (2005a) Prefrontal TMS produces smaller EEG responses than motor-cortex TMS: implications for rTMS treatment in depression. Psychopharmacology (Berl) 181:16-20.

Kahkonen S, Komssi S, Wilenius J, Ilmoniemi RJ (2005b) Prefrontal transcranial magnetic stimulation produces intensity-dependent EEG responses in humans. Neuroimage 24:955-960.

Kammer T (1999) Phosphenes and transient scotomas induced by magnetic stimulation of the occipital lobe: their topographic relationship. Neuropsychologia 37:191-198.

Kammer T, Beck S, Erb M, Grodd W (2001) The influence of current direction on phosphene thresholds evoked by transcranial magnetic stimulation. Clin Neurophysiol 112:2015-2021. 
Kandel ER, Schwartz JH, Jessell TM (2000) Principles of neural science, 4th Edition. New York: McGraw-Hill Health Professions Division.

Kastner S, Demmer I, Ziemann U (1998) Transient visual field defects induced by transcranial magnetic stimulation over human occipital pole. Exp Brain Res 118:19-26.

Kemna LJ, Gembris D (2003) Repetitive transcranial magnetic stimulation induces different responses in different cortical areas: a functional magnetic resonance study in humans. Neurosci Lett 336:85-88.

Kenet T, Bibitchkov D, Tsodyks M, Grinvald A, Arieli A (2003) Spontaneously emerging cortical representations of visual attributes. Nature 425:954-956.

Kiers L, Cros D, Chiappa KH, Fang J (1993) Variability of motor potentials evoked by transcranial magnetic stimulation. Electroencephalogr Clin Neurophysiol 89:415-423.

Kilner JM, Alonso-Alonso M, Fisher R, Lemon RN (2002) Modulation of synchrony between single motor units during precision grip tasks in humans. J Physiol 541:937-948.

King PLJ, Chiappa KH (1989) Motor evoked potentials. In: Evoked Potentials in Clinical Medicine, 2nd Edition (Chiappa KH, ed), pp 509-561. New York: Raven Press Ltd.

Kirkwood A, Bear MF (1994) Hebbian synapses in visual cortex. J Neurosci 14:16341645.

Klein T, Magerl W, Hopf HC, Sandkuhler J, Treede RD (2004) Perceptual correlates of nociceptive long-term potentiation and long-term depression in humans. $\mathrm{J}$ Neurosci 24:964-971.

Knecht S, Sommer J, Deppe M, Steinstrater O (2005) Scalp position and efficacy of transcranial magnetic stimulation. Clin Neurophysiol 116:1988-1993.

Kobayashi K, James CJ, Nakahori T, Akiyama T, Gotman J (1999) Isolation of epileptiform discharges from unaveraged EEG by independent component analysis. Clin Neurophysiol 110:1755-1763.

Koch G, Franca M, Del Olmo MF, Cheeran B, Milton R, Alvarez Sauco M, Rothwell JC (2006) Time course of functional connectivity between dorsal premotor and contralateral motor cortex during movement selection. J Neurosci 26:7452-7459. 
Komssi S, Kahkonen S (2006) The novelty value of the combined use of electroencephalography and transcranial magnetic stimulation for neuroscience research. Brain Res Brain Res Rev 52:183-192.

Komssi S, Kahkonen S, Ilmoniemi RJ (2004) The effect of stimulus intensity on brain responses evoked by transcranial magnetic stimulation. Hum Brain Mapp 21:154-164.

Komssi S, Aronen HJ, Huttunen J, Kesaniemi M, Soinne L, Nikouline VV, Ollikainen M, Roine RO, Karhu J, Savolainen S, Ilmoniemi RJ (2002) Ipsi- and contralateral EEG reactions to transcranial magnetic stimulation. Clin Neurophysiol 113:175-184.

Kuhn AA, Williams D, Kupsch A, Limousin P, Hariz M, Schneider GH, Yarrow K, Brown P (2004) Event-related beta desynchronization in human subthalamic nucleus correlates with motor performance. Brain 127:735-746.

Kuhn AA, Doyle L, Pogosyan A, Yarrow K, Kupsch A, Schneider GH, Hariz MI, Trottenberg T, Brown P (2006) Modulation of beta oscillations in the subthalamic area during motor imagery in Parkinson's disease. Brain 129:695706.

Kujirai K, Kujirai T, Sinkjaer T, Rothwell JC (2006) Associative Plasticity In Human Motor Cortex Under Voluntary Muscle Contraction. J Neurophysiol.

Lachaux JP, Lutz A, Rudrauf D, Cosmelli D, Le Van Quyen M, Martinerie J, Varela F (2002) Estimating the time-course of coherence between single-trial brain signals: an introduction to wavelet coherence. Neurophysiol Clin 32:157-174.

Lee D (2004) Behavioral context and coherent oscillations in the supplementary motor area. J Neurosci 24:4453-4459.

Leocani L, Toro C, Manganotti P, Zhuang P, Hallett M (1997) Event-related coherence and event-related desynchronization/synchronization in the $10 \mathrm{~Hz}$ and $20 \mathrm{~Hz}$ EEG during self-paced movements. Electroencephalogr Clin Neurophysiol 104:199-206.

Levy WB, Steward O (1983) Temporal contiguity requirements for long-term associative potentiation/depression in the hippocampus. Neuroscience 8:791797.

Li X, Teneback CC, Nahas Z, Kozel FA, Large C, Cohn J, Bohning DE, George MS (2004) Interleaved transcranial magnetic stimulation/functional MRI confirms that lamotrigine inhibits cortical excitability in healthy young men. Neuropsychopharmacology 29:1395-1407. 
Liepert J, Classen J, Cohen LG, Hallett M (1998) Task-dependent changes of intracortical inhibition. Exp Brain Res 118:421-426.

Loader C (1999) Local regression and likelihood. New York: Springer.

Lorch RF, Jr., Myers JL (1990) Regression Analyses of Repeated Measures Data in Cognitive Research. Journal of Experimental Psychology: Learning, Memory, \& Cognition 16:149-157.

Lynch GS, Dunwiddie T, Gribkoff V (1977) Heterosynaptic depression: a postsynaptic correlate of long-term potentiation. Nature 266:737-739.

Magistris MR, Rosler KM, Truffert A, Myers JP (1998) Transcranial stimulation excites virtually all motor neurons supplying the target muscle. A demonstration and a method improving the study of motor evoked potentials. Brain 121 ( Pt 3):437-450.

Makeig S, Jung TP, Bell AJ, Ghahremani D, Sejnowski TJ (1997) Blind separation of auditory event-related brain responses into independent components. Proc Natl Acad Sci U S A 94:10979-10984.

Makeig S, Westerfield M, Jung TP, Enghoff S, Townsend J, Courchesne E, Sejnowski TJ (2002) Dynamic brain sources of visual evoked responses. Science 295:690-694.

Malenka RC, Bear MF (2004) LTP and LTD: an embarrassment of riches. Neuron 44:5-21.

Mao JB, Evinger C (2001) Long-term potentiation of the human blink reflex. J Neurosci 21:RC151.

Maris E (2004) Randomization tests for ERP topographies and whole spatiotemporal data matrices. Psychophysiology 41:142-151.

Maris E, Oostenveld R (2006) Nonparametric Statistical Tests for EEG- and MEGdata. Neuroimage Submitted.

Markram H, L bke J, Frotscher M, Sakmann B (1997) Regulation of synaptic efficacy by coincidence of postsynaptic APs and EPSPs. Science 275:213-215.

Martin SJ, Grimwood PD, Morris RG (2000) Synaptic plasticity and memory: an evaluation of the hypothesis. Annu Rev Neurosci 23:649-711.

Martin WH, Pratt H, Schwegler JW (1995) The origin of the human auditory brainstem response wave II. Electroencephalogr Clin Neurophysiol 96:357-370.

Massimini M, Ferrarelli F, Huber R, Esser SK, Singh H, Tononi G (2005a) Breakdown of cortical effective connectivity during sleep. Science 309:22282232. 
Massimini M, Ferrarelli F, Peterson MJ, Esser SK, Huber R, Lazar M, Alexander A, Tononi G (2005b) Cortical EEG response toTMS in schizophrenia patients and normal controls. In: Society for Neuroscience, p Program No. 443.445. Washington, DC.

Mauguiere F, Allison T, Babiloni C, Buchner H, Eisen AA, Goodin DS, Jones SJ, Kakigi R, Matsuoka S, Nuwer M, Rossini PM, Shibasaki H (1999) Somatosensory evoked potentials. The International Federation of Clinical Neurophysiology. Electroencephalogr Clin Neurophysiol Suppl 52:79-90.

Michel CM, Murray MM, Lantz G, Gonzalez S, Spinelli L, Grave de Peralta R (2004) EEG source imaging. Clin Neurophysiol 115:2195-2222.

Miltner W, Braun C, Johnson R, Jr., Simpson GV, Ruchkin DS (1994) A test of brain electrical source analysis (BESA): a simulation study. Electroencephalogr Clin Neurophysiol 91:295-310.

Mima T, Hallett M (1999) Corticomuscular coherence: a review. J Clin Neurophysiol 16:501-511.

Mima T, Steger J, Schulman AE, Gerloff C, Hallett M (2000) Electroencephalographic measurement of motor cortex control of muscle activity in humans. Clin Neurophysiol 111:326-337.

Mishory A, Molnar C, Koola J, Li X, Kozel FA, Myrick H, Stroud Z, Nahas Z, George MS (2004) The maximum-likelihood strategy for determining transcranial magnetic stimulation motor threshold, using parameter estimation by sequential testing is faster than conventional methods with similar precision. J Ect 20:160-165.

Mitra PP, Pesaran B (1999) Analysis of dynamic brain imaging data. Biophys J 76:691-708.

Mitzdorf U (1985) Current source-density method and application in cat cerebral cortex: investigation of evoked potentials and EEG phenomena. Physiol Rev 65:37-100.

Moosavi SH, Ellaway PH, Catley M, Stokes MJ, Haque N (1999) Corticospinal function in severe brain injury assessed using magnetic stimulation of the motor cortex in man. J Neurol Sci 164:179-186.

Morgante F, Espay AJ, Gunraj C, Lang AE, Chen R (2006) Motor cortex plasticity in Parkinson's disease and levodopa-induced dyskinesias. Brain 129:1059-1069. 
Morris RG, Anderson E, Lynch GS, Baudry M (1986) Selective impairment of learning and blockade of long-term potentiation by an N-methyl-D-aspartate receptor antagonist, AP5. Nature 319:774-776.

Mosher JC, Lewis PS, Leahy RM (1992) Multiple dipole modeling and localization from spatio-temporal MEG data. IEEE Trans Biomed Eng 39:541-557.

Muellbacher W, Ziemann U, Boroojerdi B, Hallett M (2000) Effects of low-frequency transcranial magnetic stimulation on motor excitability and basic motor behavior. Clin Neurophysiol 111:1002-1007.

Mulert C, Jager L, Pogarell O, Bussfeld P, Schmitt R, Juckel G, Hegerl U (2002) Simultaneous ERP and event-related fMRI: focus on the time course of brain activity in target detection. Methods Find Exp Clin Pharmacol 24 Suppl D:1720.

Mulkey RM, Malenka RC (1992) Mechanisms underlying induction of homosynaptic long-term depression in area CA1 of the hippocampus. Neuron 9:967-975.

Murthy VN, Fetz EE (1992) Coherent 25- to $35-\mathrm{Hz}$ oscillations in the sensorimotor cortex of awake behaving monkeys. Proc Natl Acad Sci U S A 89:5670-5674.

Nahas Z, Lomarev M, Roberts DR, Shastri A, Lorberbaum JP, Teneback C, McConnell K, Vincent DJ, Li X, George MS, Bohning DE (2001) Unilateral left prefrontal transcranial magnetic stimulation (TMS) produces intensitydependent bilateral effects as measured by interleaved BOLD fMRI. Biol Psychiatry 50:712-720.

Nelder J, Mead R (1965) A simplex method for function minimization. Computer Journal 7:308-313.

Nelson SB, Turrigiano GG (1998) Synaptic depression: a key player in the cortical balancing act. Nat Neurosci 1:539-541.

Neuper C, Pfurtscheller G (2001) Event-related dynamics of cortical rhythms: frequency-specific features and functional correlates. Int J Psychophysiol 43:41-58.

Nichols TE, Holmes AP (2002) Nonparametric permutation tests for functional neuroimaging: a primer with examples. Hum Brain Mapp 15:1-25.

Niedermeyer E (1999) The normal EEG of the waking adult. In: Electroencephalography. Basic principles, clinical applications and related fields., 4 Edition (Niedermeyer E, Lopes da Silva F, eds), pp 149-173. Baltimore: Williams and Wilkins. 
Nitsche MA, Paulus W (2000) Excitability changes induced in the human motor cortex by weak transcranial direct current stimulation. J Physiol 527 Pt 3:633639.

Nunez PL (1981) Electric fields of the brain : the neurophysics of EEG. New York: Oxford University Press.

Nunez PL, Silberstein RB (2000) On the relationship of synaptic activity to macroscopic measurements: does co-registration of EEG with fMRI make sense? Brain Topogr 13:79-96.

Nuwer MR, Lehmann D, Lopes da Silva F, Matsuoka S, Sutherling W, Vibert JF (1994a) IFCN guidelines for topographic and frequency analysis of EEGs and EPs. Report of an IFCN committee. International Federation of Clinical Neurophysiology. Electroencephalogr Clin Neurophysiol 91:1-5.

Nuwer MR, Aminoff M, Desmedt J, Eisen AA, Goodin D, Matsuoka S, Mauguiere F, Shibasaki H, Sutherling W, Vibert JF (1994b) IFCN recommended standards for short latency somatosensory evoked potentials. Report of an IFCN committee. International Federation of Clinical Neurophysiology. Electroencephalogr Clin Neurophysiol 91:6-11.

Okamoto M, Dan H, Sakamoto K, Takeo K, Shimizu K, Kohno S, Oda I, Isobe S, Suzuki T, Kohyama K, Dan I (2004) Three-dimensional probabilistic anatomical cranio-cerebral correlation via the international 10-20 system oriented for transcranial functional brain mapping. Neuroimage 21:99-111.

Oliviero A, Strens LH, Di Lazzaro V, Tonali PA, Brown P (2003) Persistent effects of high frequency repetitive TMS on the coupling between motor areas in the human. Exp Brain Res 149:107-113.

Oostenveld R, Praamstra P (2001) The five percent electrode system for highresolution EEG and ERP measurements. Clin Neurophysiol 112:713-719.

Pascual Leone A, Walsh V, Rothwell J (2000) Transcranial magnetic stimulation in cognitive neuroscience--virtual lesion, chronometry, and functional connectivity. Current Opinion in Neurobiology 10:232-237.

Pascual Marqui RD, Michel CM, Lehmann D (1994) Low resolution electromagnetic tomography: a new method for localizing electrical activity in the brain. International Journal of Psychophysiology 18:49-65.

Pascual-Leone A, Gates JR, Dhuna A (1991) Induction of speech arrest and counting errors with rapid-rate transcranial magnetic stimulation. Neurology 41:697702. 
Pascual-Leone A, Valls-Sole J, Wassermann EM, Hallett M (1994) Responses to rapid-rate transcranial magnetic stimulation of the human motor cortex. Brain 117 ( Pt 4):847-858.

Pascual-Marqui R (1999) Review of Methods for Solving the EEG Inverse Problem. International Journal of Bioelectromagnetism 1:75-86.

Paus T, Sipila PK, Strafella AP (2001a) Synchronization of neuronal activity in the human primary motor cortex by transcranial magnetic stimulation: an EEG study. J Neurophysiol 86:1983-1990.

Paus T, Castro-Alamancos MA, Petrides M (2001b) Cortico-cortical connectivity of the human mid-dorsolateral frontal cortex and its modulation by repetitive transcranial magnetic stimulation. Eur J Neurosci 14:1405-1411.

Pfurtscheller G, Lopes da Silva FH (1999) Event-related EEG/MEG synchronization and desynchronization: basic principles. Clin Neurophysiol 110:1842-1857.

Pfurtscheller G, Woertz M, Supp G, Lopes da Silva FH (2003) Early onset of postmovement beta electroencephalogram synchronization in the supplementary motor area during self-paced finger movement in man. Neurosci Lett 339:111114.

Pitcher JB, Ogston KM, Miles TS (2003) Age and sex differences in human motor cortex input-output characteristics. J Physiol 546:605-613.

Pleger B, Dinse HR, Ragert P, Schwenkreis P, Malin JP, Tegenthoff M (2001) Shifts in cortical representations predict human discrimination improvement. Proc Natl Acad Sci U S A 98:12255-12260.

Pleger B, Foerster AF, Ragert P, Dinse HR, Schwenkreis P, Malin JP, Nicolas V, Tegenthoff M (2003) Functional imaging of perceptual learning in human primary and secondary somatosensory cortex. Neuron 40:643-653.

Pleger B, Blankenburg F, Bestmann S, Ruff C, Wiech K, Stephan K, Friston K, Dolan R (2006) Repetitive Transcranial Magnetic Stimulation-Induced Changes in Sensorimotor Coupling Parallel Improvements of Somatosensation in Humans. J Neurosci 26:1945-1952.

Ploner M, Gross J, Timmermann L, Pollok B, Schnitzler A (2006) Oscillatory activity reflects the excitability of the human somatosensory system. Neuroimage.

Poline J (2003) Contrasts and classical inference. In: Human Brain Function, 2nd Edition (Frackowiak R, Friston K, eds): Academic Press. 
Quartarone A, Bagnato S, Rizzo V, Siebner HR, Dattola V, Scalfari A, Morgante F, Battaglia F, Romano M, Girlanda P (2003) Abnormal associative plasticity of the human motor cortex in writer's cramp. Brain 126:2586-2596.

Ragert P, Kalisch T, Dinse HR (2005) Perceptual changes in human tactile discrimination behavior induced by coactivation using LTP and LTD protocols. In: Society for Neuroscience, p Program No. 173.176. Washington, DC.

Rappelsberger P (1998) Probability mapping of amplitude and coherence: technical aspects. In: EEG and Thinking (Petsche H, Etlinger C, eds), pp 63-78. Vienna: Austrian Academy of Sciences.

Rau C, Plewnia C, Hummel F, Gerloff C (2003) Event-related desynchronization and excitability of the ipsilateral motor cortex during simple self-paced finger movements. Clin Neurophysiol 114:1819-1826.

Ravazzani P, Tognola G, Grandori F, Budai R, Locatelli T, Cursi M, Di Benedetto G, Comi G (1995) Temporal segmentation and multiple-source analysis of shortlatency median nerve SEPs. J Med Eng Technol 19:70-76.

Restuccia D, Valeriani M, Grassi E, Gentili G, Mazza S, Tonali P, Mauguiere F (2002) Contribution of GABAergic cortical circuitry in shaping somatosensory evoked scalp responses: specific changes after single-dose administration of tiagabine. Clin Neurophysiol 113:656-671.

Ridding MC, Taylor JL (2001) Mechanisms of motor-evoked potential facilitation following prolonged dual peripheral and central stimulation in humans. J Physiol 537:623-631.

Rossini PM, Rossi S, Pasqualetti P, Tecchio F (1999) Corticospinal excitability modulation to hand muscles during movement imagery. Cereb Cortex 9:161167.

Rossini PM, Barker AT, Berardelli A, Caramia MD, Caruso G, Cracco RQ, Dimitrijevic MR, Hallett M, Katayama Y, Lucking CH, et al. (1994) Noninvasive electrical and magnetic stimulation of the brain, spinal cord and roots: basic principles and procedures for routine clinical application. Report of an IFCN committee. Electroencephalogr Clin Neurophysiol 91:79-92.

Royer S, Pare D (2003) Conservation of total synaptic weight through balanced synaptic depression and potentiation. Nature 422:518-522.

Ruohonen J, Ilmoniemi RJ (1999) Modeling of the stimulating field generation in TMS. Electroencephalogr Clin Neurophysiol Suppl 51:30-40. 
Ruohonen JO, Ravazzani P, Ilmoniemi RJ, Galardi G, Nilsson J, Panizza M, Amadio S, Grandori F, Comi G (1996) Motor cortex mapping with combined MEG and magnetic stimulation. Electroencephalogr Clin Neurophysiol Suppl 46:317-322.

Salmelin R, Hamalainen M, Kajola M, Hari R (1995) Functional segregation of movement-related rhythmic activity in the human brain. Neuroimage 2:237243.

Sanes JN, Donoghue JP (1993) Oscillations in local field potentials of the primate motor cortex during voluntary movement. Proc Natl Acad Sci U S A 90:44704474.

Scanziani M, Malenka RC, Nicoll RA (1996) Role of intercellular interactions in heterosynaptic long-term depression. Nature 380:446-450.

Scherg M, Von Cramon D (1986) Evoked dipole source potentials of the human auditory cortex. Electroencephalogr Clin Neurophysiol 65:344-360.

Scherg M, von Cramon D (1986) Psychoacoustic and electrophysiologic correlates of central hearing disorders in man. Eur Arch Psychiatry Neurol Sci 236:56-60.

Scherg M, Berg P (1996) New concepts of brain source imaging and localization. Electroencephalogr Clin Neurophysiol Suppl 46:127-137.

Scherg M, Hoechstetter K (2000) Tutorial 1: Getting started with EEG simulations. In: BESA software documentation.

Scherg M, Hoechstetter K, Berg P (2004) BESA - Brain Electrical Source Analysis. In. Graefelfing/Munich.

Scherg M, Ille N, Bornfleth H, Berg P (2002) Advanced tools for digital EEG review: virtual source montages, whole-head mapping, correlation, and phase analysis. J Clin Neurophysiol 19:91-112.

Schnitzler A, Gross J (2005) Normal and pathological oscillatory communication in the brain. Nat Rev Neurosci 6:285-296.

Schnitzler A, Timmermann L, Gross J (2006) Physiological and pathological oscillatory networks in the human motor system. J Physiol Paris 99:3-7.

Schoffelen JM, Oostenveld R, Fries P (2005) Neuronal coherence as a mechanism of effective corticospinal interaction. Science 308:111-113.

Schorr A, Ellrich J (2002) Long-term depression of the human blink reflex. Exp Brain Res 147:549-553. 
Shadlen MN, Newsome WT (1998) The variable discharge of cortical neurons: implications for connectivity, computation, and information coding. J Neurosci 18:3870-3896.

Shah AS, Bressler SL, Knuth KH, Ding M, Mehta AD, Ulbert I, Schroeder CE (2004) Neural dynamics and the fundamental mechanisms of event-related brain potentials. Cereb Cortex 14:476-483.

Shahaf G, Marom S (2001) Learning in networks of cortical neurons. The Journal of Neuroscience 21:8782-8788.

Shimazu H, Maier MA, Cerri G, Kirkwood PA, Lemon RN (2004) Macaque ventral premotor cortex exerts powerful facilitation of motor cortex outputs to upper limb motoneurons. J Neurosci 24:1200-1211.

Shulz DE, Brasier D, Feldman DE (2004) Spike-Timing Dependent Plasticity Investigated Using Whole-Cell Recording In Rat Somatosensory Cortex (S1) In Vivo. In: Society for Neuroscience, p Program No. 57.51. Washington, DC.

Slepian D, Pollak H (1961) Prolate spheroidal wavefunctions Fourier analysis and uncertainty. I. Bell Sys Tech J 40:43-63.

Sporns O, Tononi G, Kotter R (2005) The human connectome: a structural description of the human brain. PLoS Comput Biol 1:e42.

Stefan K, Wycislo M, Classen J (2004) Modulation of associative human motor cortical plasticity by attention. J Neurophysiol 92:66-72.

Stefan K, Kunesch E, Cohen LG, Benecke R, Classen J (2000) Induction of plasticity in the human motor cortex by paired associative stimulation. Brain $123 \mathrm{Pt}$ 3:572-584.

Stefan K, Kunesch E, Benecke R, Cohen LG, Classen J (2002) Mechanisms of enhancement of human motor cortex excitability induced by interventional paired associative stimulation. J Physiol 543:699-708.

Stefan K, Wycislo M, Gentner R, Schramm A, Naumann M, Reiners K, Classen J (2005) Temporary Occlusion of Associative Motor Cortical Plasticity by Prior Dynamic Motor Training. Cereb Cortex.

Strens LH, Oliviero A, Bloem BR, Gerschlager W, Rothwell JC, Brown P (2002) The effects of subthreshold $1 \mathrm{~Hz}$ repetitive TMS on cortico-cortical and interhemispheric coherence. Clin Neurophysiol 113:1279-1285.

Takashima A, Jensen O, Oostenveld R, Maris E, van de Coevering M, Fernandez G (2006) Successful declarative memory formation is associated with ongoing activity during encoding in a distributed neocortical network related to 
working memory: a magnetoencephalography study. Neuroscience 139:291297.

Talairach J, Tournoux P (1988) Co-planar stereotaxic atlas of the human brain : an approach to medical cerebral imaging. Stuttgart ; New York

New York: G. Thieme ;

Thieme Medical Publishers.

Tegenthoff M, Ragert P, Pleger B, Schwenkreis P, Forster AF, Nicolas V, Dinse HR (2005) Improvement of tactile discrimination performance and enlargement of cortical somatosensory maps after 5 Hz rTMS. PLoS Biol 3:e362.

Teyler TJ, Hamm JP, Clapp WC, Johnson BW, Corballis MC, Kirk IJ (2005) Longterm potentiation of human visual evoked responses. Eur J Neurosci 21:20452050.

Thickbroom GW, Byrnes ML, Mastaglia FL (1999) A model of the effect of MEP amplitude variation on the accuracy of TMS mapping. Clin Neurophysiol 110:941-943.

Thielscher A, Kammer T (2002) Linking physics with physiology in TMS: a sphere field model to determine the cortical stimulation site in TMS. Neuroimage 17:1117-1130.

Thomson DJ (1982) Spectrum estimation and harmonic analysis. Proceedings of the IEEE 70:1055-1095.

Thut G, Ives JR, Kampmann F, Pastor MA, Pascual-Leone A (2005) A new device and protocol for combining TMS and online recordings of EEG and evoked potentials. J Neurosci Methods 141:207-217.

Thut G, Northoff G, Ives JR, Kamitani Y, Pfennig A, Kampmann F, Schomer DL, Pascual-Leone A (2003) Effects of single-pulse transcranial magnetic stimulation (TMS) on functional brain activity: a combined event-related TMS and evoked potential study. Clin Neurophysiol 114:2071-2080.

Tiitinen H, Virtanen J, Ilmoniemi RJ, Kamppuri J, Ollikainen M, Ruohonen J, Naatanen R (1999) Separation of contamination caused by coil clicks from responses elicited by transcranial magnetic stimulation. Clin Neurophysiol 110:982-985.

Tikhonov AN, Arsenin (1977) Solutions of ill-posed problems. Washington

New York: Winston; distributed solely by Halsted Press. 
Tsuji S, Murai Y (1986) Scalp topography and distribution of cortical somatosensory evoked potentials to median nerve stimulation. Electroencephalogr Clin Neurophysiol 65:429-439.

Ueki Y, Mima T, Kotb MA, Sawada H, Saiki H, Ikeda A, Begum T, Reza F, Nagamine T, Fukuyama H (2006) Altered plasticity of the human motor cortex in Parkinson's disease. Ann Neurol 59:60-71.

van der Kamp W, Zwinderman AH, Ferrari MD, van Dijk JG (1996) Cortical excitability and response variability of transcranial magnetic stimulation. J Clin Neurophysiol 13:164-171.

Van Der Werf YD, Paus T (2006) The neural response to transcranial magnetic stimulation of the human motor cortex. I. Intracortical and cortico-cortical contributions. Exp Brain Res.

Vanderzant CW, Beydoun AA, Domer PA, Hood TW, Abou-Khalil BW (1991) Polarity reversal of N20 and P23 somatosensory evoked potentials between scalp and depth recordings. Electroencephalogr Clin Neurophysiol 78:234239.

Vigario R, Sarela J, Jousmaki V, Hamalainen M, Oja E (2000) Independent component approach to the analysis of EEG and MEG recordings. IEEE Trans Biomed Eng 47:589-593.

Virtanen J, Ruohonen J, Naatanen R, Ilmoniemi RJ (1999) Instrumentation for the measurement of electric brain responses to transcranial magnetic stimulation. Med Biol Eng Comput 37:322-326.

Vitacco D, Brandeis D, Pascual-Marqui R, Martin E (2002) Correspondence of eventrelated potential tomography and functional magnetic resonance imaging during language processing. Hum Brain Mapp 17:4-12.

Walsh V, Rushworth M (1999) A primer of magnetic stimulation as a tool for neuropsychology. Neuropsychologia 37:125-135.

Walsh V, Ashbridge E, Cowey A (1998a) Cortical plasticity in perceptual learning demonstrated by transcranial magnetic stimulation. Neuropsychologia 36:4549.

Walsh V, Ellison A, Battelli L, Cowey A (1998b) Task-specific impairments and enhancements induced by magnetic stimulation of human visual area V5. Proc R Soc Lond B Biol Sci 265:537-543.

Wassermann EM (1998) Risk and safety of repetitive transcranial magnetic stimulation: report and suggested guidelines from the International Workshop 
on the Safety of Repetitive Transcranial Magnetic Stimulation, June 5-7, 1996. Electroencephalogr Clin Neurophysiol 108:1-16.

Wassermann EM, Lisanby SH (2001) Therapeutic application of repetitive transcranial magnetic stimulation: a review. 112:1367-1377.

Wassermann EM, Grafman J, Berry C, Hollnagel C, Wild K, Clark K, Hallett M (1996) Use and safety of a new repetitive transcranial magnetic stimulator. Electroencephalogr Clin Neurophysiol 101:412-417.

Webb BS, Dhruv NT, Solomon SG, Tailby C, Lennie P (2005) Early and late mechanisms of surround suppression in striate cortex of macaque. J Neurosci 25:11666-11675.

Wiesendanger M, Miles TS (1982) Ascending pathway of low-threshold muscle afferents to the cerebral cortex and its possible role in motor control. Physiol Rev 62:1234-1270.

Williams ER, Baker SN (2005) Towards an Understanding of Corticomuscular Coherence: a Realistic Computational Model. In: Society for Neuroscience, $p$ Program No. 628.625. Washington DC: Online.

Wolters A, Sandbrink F, Schlottmann A, Kunesch E, Stefan K, Cohen LG, Benecke R, Classen J (2003) A temporally asymmetric Hebbian rule governing plasticity in the human motor cortex. J Neurophysiol 89:2339-2345.

Wolters A, Schmidt A, Schramm A, Zeller D, Naumann M, Kunesch E, Benecke R, Reiners K, Classen J (2005) Timing-dependent plasticity in human primary somatosensory cortex. J Physiol 565:1039-1052.

Woodforth IJ, Hicks RG, Crawford MR, Stephen JP, Burke DJ (1996) Variability of motor-evoked potentials recorded during nitrous oxide anesthesia from the tibialis anterior muscle after transcranial electrical stimulation. Anesth Analg 82:744-749.

Yao H, Dan Y (2001) Stimulus timing-dependent plasticity in cortical processing of orientation. Neuron 32:315-323.

Yousry TA, Schmid UD, Alkadhi H, Schmidt D, Peraud A, Buettner A, Winkler P (1997) Localization of the motor hand area to a knob on the precentral gyrus. A new landmark. Brain 120 ( Pt 1):141-157.

Zaaroor M, Pratt H, Starr A (2001) Influence of task-related ipsilateral hand movement on motor cortex excitability. Clin Neurophysiol 112:908-916.

Zaaroor M, Pratt H, Starr A (2003) Time course of motor excitability before and after a task-related movement. Neurophysiol Clin 33:130-137. 
Zhang LI, Tao HW, Holt CE, Harris W, Poo M (1998) A critical window for cooperation and competition among developing retinocortical synapses. Nature 395:37-44.

Ziemann U, Muellbacher W, Hallett M, Cohen LG (2001) Modulation of practicedependent plasticity in human motor cortex. Brain 124:1171-1181.

Ziemann U, Ilic TV, Pauli C, Meintzschel F, Ruge D (2004) Learning modifies subsequent induction of long-term potentiation-like and long-term depressionlike plasticity in human motor cortex. J Neurosci 24:1666-1672. 